\title{
CELLULAR INFLAMMATION IN MODELS OF ACUTE GOUT
}

By

William John Martin

The Malaghan Institute of Medical Research Wellington, New Zealand

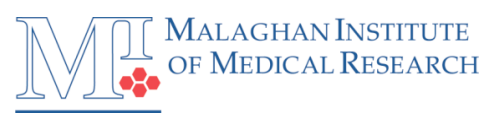

\begin{abstract}
A thesis
submitted to the Victoria University of Wellington in fulfilment of the requirements for the degree of Doctor of Philosophy In Cellular Biology
\end{abstract}

Victoria University of Wellington 2008 


\section{Acknowledgements}

How did I get myself into all this trouble.

My first thanks is to my family. My family has a particular aptitude for reproduction that has skipped me. My mother has eight children, my father, another six. My mother is one of eight, and my father one of thirteen. A talent for fertility has not escaped my siblings. It has always been a relief to disappear into tribe of caring family members.

For all of our number, I am the first of my family to undertake a PhD. A PhD in science is an unusual endeavour for Maori; at least, for Maori to whom I have spoken, because the isolating nature of the work requires the sacrifice of a type of interconnectivity that is so inherent to our spirituality. Invisibility in the Maori community was one of the prices that had to be paid to accomplish this $\mathrm{PhD}$. It was definitely worth it.

I want to thank my supervisor Jacquie Harper. She was extremely liberal with her time, effort and support. She also has a silver tongue, an ability that has evaded her first $\mathrm{PhD}$ student; although I try to give it a good attempt. I am extremely appreciative for all the support, time, patience and feedback that she has given me.

In the lab, I especially want to thank Elizabeth Chia whose (affectionally) autocratic and workaholic tendencies made lab-life run smoothly, and who always embraced conversational topics based on bizarre bodily functions without demur. Big thank yous to all other members of the Arthritis Group: Rebecca Grainger, Mischa Walton, Tommy Liu, Stefanie Steiger and erstwhile members Kylie Price, Oliver Chow Worn and Rene $\mathrm{M}^{\mathrm{c} L a u g h l i n .}$ Thanks to flow cytometry experts Kylie Price and Joanna Roberts who performed the cell sorting for me. Thanks also to my proofreaders: Patries Herst, Rebecca Grainger, Liz Forbes, Kathryn Farrand and Lisa Goldsack. Meto Leach for all the lunches, keeping an eye out, and throwing a laptop my way. The consultation room at the Malaghan Institute also deserves a special mention, as that bed has saved me 
during many an overnight experiment that required freakish hours. I still jump a little when I hear the word "timecourse."

There have been some people that have had a great impact on me over my $\mathrm{PhD}$ studies. Patries, who has been a friend and mentor in many enlightening adventures that would seem gauche and perplexing to most other people. Nina Dickgebber who, along with many more important things, plied me with hazelnut Knoppers and Milka on the home stretch and snapped me out of stress with a nice holiday. Kathryn Farrand, for sourcing my compulsive addiction to the various TV series that helped to demolish the white noise in the solitary confinement of my bedroom while writing. Rebecca Grainger for introducing me to This American Life and for the general reflections on life. Apii for the post-midnight meals, chats, and drive homes during long lab nights. Alicia Ingruber for her groove. Tesi Leota (Brain) for turning up, at a moment that really made a difference, in the form of a voice when the body was hindered. Hiirini Pomare, Pauline Harris, Christina (Kiritina) Gonzalez and Alanah Woodland, my hangout fellows of indigenous stock.

The mettle of my body has been synthesised from a raw starting material of chocolate. So, thank you to the chocolates of the world of all textures, colours, and cocoa percentages. I am not prejudiced; all chocolates belong in my world.

I love it when people give me money. So big acknowledgements to the Foundation for Research, Science and Technology, Victoria University Completion Scholarships, the Maori Education Trust, the Royal Society, Australasian Society of Immunologists NZ, and my supervisor Jacquie Harper for the financial support given over the period of my PhD. Also thank you to Te Ropu Awhina Putaiao (headed by the illustrious Liz Richardson) as well as Nga Pae o te Maramatanga, for providing Maori and Pacific Nations' academic networks. He aha te kai a te Rangatira? Ko te korero! 
Again, thanks to my family, the Martin Tribe, and in particular to my Mum, Yvonne for being strong while bringing up eight children on her own. We may not have had much money, but she taught us what it meant to live a good life.

Finally, I don't know what the organizing force of life is, but I have a strong suspicion that it is always at work in my life. I'm very grateful to it, whatever it is. 


\section{Abstract}

Gout is a common form of inflammatory arthritis that is caused by the precipitation of monosodium urate crystals (MSU) in the joints. The acute form of gout is associated with sudden painful inflammatory episodes characterised by a large infiltration of neutrophils. The involvement of monocytes and macrophages is also recognised to be important, although the exact roles of monocytes and macrophages in gout need to be clarified. The overall objective of this thesis was to investigate the contributions of neutrophils, monocytes and macrophages to acute gouty inflammation.

To determine whether the presence of other cells may be affecting neutrophil activation in gout inflammation, human blood neutrophils were stimulated with MSU as a purified population and in a mixed white blood cell population. The half-life of neutrophils in culture increased from $10 \mathrm{~h}$ in purified neutrophil cultures to $>24 \mathrm{~h}$ in mixed cell cultures. The increase in viability was associated with large increases in cytokine production (TNF $\alpha$, IL-1 $\beta$, IL-6, IL-8) in mixed cell cultures. Exposure of neutrophils to media conditioned by MSU-stimulated mononuclear cells improved both neutrophil viability and stimulated the IL-8 production from neutrophils to a greater extent than direct contact with MSU. Exposure to conditioned media also primed MSU-stimulated neutrophil superoxide responses. High superoxide production was also observed when serum was lowered to $<10 \%$. These results indicate that neutrophil activation in gout largely occurs via soluble factors present in the pro-inflammatory environment, rather than by direct contact with MSU alone.

Polygodial, a compound derived from the plant, Horopito (Pseudowintera colorata), and a number of structurally related sesquiterpene dialdehydes were tested for their potential to suppress neutrophil activation in gout. Polygodial inhibited MSU- and PMAstimulated neutrophil superoxide production in vitro with $\mathrm{IC}_{50}$ values of $0.78 \mu \mathrm{M}$ and $0.16 \mu \mathrm{M}$ respectively. This activity was largely dependent on the dialdehyde functionality but was enhanced by the presence of ring hydroxyl groups. The removal of hydroxyl groups was also associated with an increased cytoxicity. Polygodial and 
two other sesquiterpene dialdehyde compounds inhibited neutrophil superoxide production in an MSU model of acute gout, while Polygodial and a second compound also inhibited neutrophil recruitment. These studies confirm Polygodial and two structurally related sesquiterpene dialdehyde as potential anti-inflammatory compounds for potential use in gout.

To confirm whether monocytes and/or macrophages were important in the onset of gout, resident macrophages and MSU-recruited monocytes were studied in a peritoneal model of MSU-induced inflammation. Gr- $1^{+}, 7 / 4^{-}$monocytes were recruited to MSU-induced inflammation within $4 \mathrm{~h}$, however high cytokine production (IL-1 $\beta$, TNF $\alpha$, IL-6, MCP1) in the peritoneum preceded peak monocyte infiltration. Infiltrating monocytes did not produce high amounts of IL-6 and TNF $\alpha$, nor were they able to produce proinflammatory cytokines when re-stimulated with MSU ex vivo. However, resident macrophages exhibited production of IL-1 $\beta$, TNF $\alpha$ and IL-6 following exposure to MSU ex vivo. Depletion of macrophages in vivo by clodronate liposomes led to a reduced recruitment of neutrophils and a lowered production of IL-1 $\beta$ and IL-6 without affecting the recruitment of monocytes. These data identify macrophages, rather than monocytes, as key cells in initiationing and driving of inflammation in acute gout.

The lack of responses from MSU-stimulated monocytes led to the question of what function, if any, was being exhibited by monocytes during MSU-induced inflammation. Therefore, the phenotype and corresponding function of monocytes was profiled over the course of MSU-induced inflammation. Newly recruited monocytes were small in size and expressed high levels of Gr-1 and 7/4, low levels of F4/80 and CD80. Over 48h the expression of Gr-1 and 7/4 was lost, while the expression of CD80 increased. These changes were associated with an increase in cell size, phagocytic capacity, and the production of pro-inflammatory cytokines in response to MSU. Changes in phenotype indicated differentiation into immature and then resident-like macrophages, and this was confirmed by PKH26-labelling infiltrating monocytes, which acquired an $\mathrm{F} 4 / 80^{\mathrm{hi}}$, resident macrophage phenotype after 3 to 5 days. Differentiation into macrophages was associated with an increased uptake of apoptotic neutrophils both in 
vivo and following ex vivo incubation. The phagocytosis of apoptotic neutrophils was associated with a reduced production of IL-1 $\beta$ following stimulation with MSU ex vivo. These results indicate that MSU-recruited monocytes are not pro-inflammatory cells in gout, rather, they differentiate into resident macrophages over 3 to 5 days, and may over time aid in the resolution of gouty inflammation through the clearance of apoptotic neutrophils, a process that also suppresses further inflammatory responses.

Together, the results of this thesis indicate that macrophages are key pro-inflammatory cells in gout contributing to neutrophil recruitment and cytokine production; that recruited neutrophils are activated by soluble mediators produced by MSU-activated cells, as well as by low serum environments; and that recruited monocytes are capable of both mediating the resolution of MSU-induced inflammation and replenishing the local resident macrophage population. These results provide a more clear model of the cellular events that occur during acute gout in humans that to date have been limited by a lack of in vivo-based studies. 


\section{Table of Contents}

Acknowledgements

Abstract $\quad$ V

Table of Contents VIII

List of Figures $\quad$ XVI

$\begin{array}{ll}\text { List of Tables } & \text { XX1 }\end{array}$

List of Abbreviations $\quad$ XXII

Chapter 1. General Introduction 1

1.1 A history of gouty disease 2

1.2 Monosodium urate crystals 3

1.3 Acute gouty arthritis 5

1.4 Chronic gout 7

1.5 Uric acid 7

1.6 Loss of uricase 9

1.7 Gout risk factors 12

$\begin{array}{ll}\text { 1.7.1 Gender } & 12\end{array}$

$\begin{array}{ll}\text { 1.7.2 Diet } & 12\end{array}$

$\begin{array}{ll}\text { 1.7.3 Genetic and race-related risk } & 13\end{array}$

$\begin{array}{ll}\text { 1.7.4 An aging population } & 14\end{array}$

$\begin{array}{ll}1.8 \text { Co-morbid diseases } & 14\end{array}$

1.9 Treatment and management of gout 15

1.9.1 Diet 15

1.9.2 Anti inflammatory drugs 16

1.9.3 Uric acid lowering drugs 16

$\begin{array}{ll}\text { 1.9.4 Limitations of current therapies } & 18\end{array}$

$\begin{array}{ll}\text { 1.10 Cellular inflammation in acute gout inflammation } & 18\end{array}$

1.10.1 Initiation of gout inflammation 19

1.10.2 The necessity of MSU recognition by immune cells as 19 
a "danger signal"

1.10.3 Innate cell recognition of MSU to induce an inflammatory response.

1.10.4 Production of cytokines by MSU-stimulated innate cells

1.10.4.1 IL-1 $\beta$ and the inflammasome 22

1.10.5 Resolution in gout 23

$\begin{array}{ll}\text { 1.10.6 Key innate cells in gout } & 24\end{array}$

$\begin{array}{ll}\text { 1.10.7 Neutrophils } & 25\end{array}$

1.10.7.1 Neutrophil recruitment 25

1.10.7.2 Recruitment of neutrophils in gout 27

1.10.7.3 Activation and survival of neutrophils in inflammation 28

1.10.7.4 Production of superoxide $\quad 29$

1.10.7.5 Collateral damage through excessive ROS production 31

1.10.8 Neutrophil investigation 31

1.10.9 The mononuclear phagocyte system 33

1.10.10 Monocytes 33

1.10.10.1 Monocytes in gout 36

1.10.11 Macrophages $\quad 39$

1.10.11.1 Macrophages in gout $\quad 40$

1.10.12 Using research models to investigate gout 40

1.10.12.1 Murine peritoneal model of gout $\quad 41$

1.10.12.2 Murine air pouch model $\quad 41$

1.11 Aims of this study $\quad 42$

Chapter 2. Materials and Methods

2.1 Reagents $\quad 44$

2.1.1 Sesquiterpene dialdehydes 46

2.2 Antibodies 46

2.3 Kits 47

$\begin{array}{ll}\text { 2.4 Disposables } & 48\end{array}$ 
2.5 Buffers and Media 48

2.6 Preparation of MSU 51

2.7 Endotoxin levels

2.7.1 Limulus amebocyte lysate (LAL) assay tested 51

2.7.2 Purchased reagents $\quad 52$

2.8 Isolation of cell types $\quad 52$

2.8.1 Human neutrophils $\quad 52$

2.8.2 Human peripheral blood mononuclear cells $\quad 52$

2.8.3 Total human white blood cells 53

2.8.4 Enrichment of naïve mouse peritoneal lymphocytes and 53 macrophages

2.8.5 Enrichment of peritoneal mast cells $\quad 54$

2.8.6 Sorting of murine leukocyte populations $\quad 54$

2.9 Cell lines and culture $\quad 54$

2.9.1 Human umbilical vein endothelial cells (HUVEC) 54

2.9.2 RAW 264.7 cells $\quad 55$

2.9.3 Culture of primary mouse mesothelial cells 55

2.10 Histology 56

2.10.1 Morphological stain for differential cell counts 56

2.10.2 Hematoxylin and Eosin (H\&E) staining of peritoneal tissue 56

2.10.3 Visualisation of MSU crystal and cell clumping 57

2.10.4 Immunofluorescent histology of resident peritoneal macrophages 57

2.10.5 Staining of visceral peritoneal tissue 58

2.11 Measurement of superoxide production $\quad 58$

2.11.1 PMA stimulation of neutrophils $\quad 58$

2.11.2 MSU stimulation of neutrophils $\quad 59$

2.11.3 Serum stimulation of neutrophils $\quad 59$

2.11.4 Superoxide production by ex vivo murine peritoneal neutrophils $\quad 60$

$\begin{array}{ll}\text { 2.11.5 Luminol chemiluminescence } & 60\end{array}$

2.12 Viability stains $\quad 61$

$\begin{array}{ll}\text { 2.12.1 Trypan blue } & 61\end{array}$ 
2.12.2 Neutrophil trypan blue assay for sesquiterpene dialdehyde compounds

2.12.3 Annexin-V and propidium iodide (PI) double staining

2.12.4 Measuring the effect of sesquiterpene dialdehyde compounds on neutrophil viability by annexin-V/PI staining

2.13 Cytokine analyses

2.14 Human neutrophil, mononuclear cell and white blood cell experiments

2.14.1 Neutrophil and WBC apoptosis and viability assay

2.14.2 Neutrophil and WBC soluble cytokine production

2.14.3 Preparation of conditioned media

2.14.4 Intracellular IL-8 staining of neutrophils

2.14.5 Measuring the effect of soluble mediators on neutrophil viability

2.14.6 Measuring the effect of soluble mediators on MSU-stimulated superoxide production

2.15 Phagocytosis assays

2.15.1 Phagocytosis of fluorescent beads

2.15.2 Peroxidase staining for phagocytosed neutrophils

2.16 Animal studies

2.16.1 Ethical approvals

2.16.2 Mice

2.16.3 MSU-induced peritonitis

2.16.4 Thioglycollate-induced peritonitis

2.16.5 Testing of sesquiterpene dialdehyde compounds in the in vivo gout model

2.16.6 PKH26 experiments

2.16.7 Preparation of clodronate-loaded liposomes

2.16.8 Clodronate liposome depletion of resident macrophages

2.17 Intracellular staining of ex vivo cells

2.18 Restimulation Assays

2.19 Whole peritoneal tissue and peritoneal leukocyte culture 
2.21 Inter-experiment variability

2.22 Image analyses and editing

Chapter 3. Neutrophil activation in MSU-induced inflammation 71

3.1 Introduction

\subsection{Results}

3.2.1 Measuring neutrophil superoxide production

3.2.2 Superoxide production by MSU-stimulated neutrophils is affected by human serum concentration.

3.2.3 The effect of MSU on neutrophil viability and IL-8 production

3.2.4 The effect of mixed cell populations on MSU-induced neutrophil activation

3.2.5 The effect of soluble mediators on neutrophil activation and function

3.2.5.1 Neutrophils survival is improved by MSU-induced soluble mediators and does not require cell-cell contact

3.2.5.2 Neutrophil IL-8 production is activated by MSU-induced soluble mediators

3.2.5.3 MSU-induced soluble mediators prime human neutrophils to produce superoxide

3.2.6 Neutrophil activation and viability in vivo

\section{Chapter 4. Inhibiting neutrophil inflammation using sesquiterpene dialdehydes inflammation 
4.2.1 Inhibition of neutrophil superoxide production by sesquiterpene dialdehydes

4.2.2 Structure-activity related inhibition of PMA-induced superoxide production

4.2.3 Structure-activity related inhibition of MSU-induced superoxide production

4.2.4 Cytotoxic activity of sesquiterpene dialdehydes

4.2.5 Inhibition of neutrophil superoxide production in vivo

4.2.6 Additional anti-inflammatory activities of sesquiterpene dialadehyde compounds

4.2.6.1 Sesquiterpene dialdehydes compounds inhibit superoxide production by endothelial cells

4.2.6.2 Sesquiterpene dialadehyde compounds inhibit pro-inflammatory cytokine production in MSU-stimulated macrophages

5.2.1 Characterising the murine peritoneal model of acute gout.

5.2.2 Production of pro-inflammatory cytokines by cells stimulated with MSU crystals

5.2.2.1 Investigating cytokine responses of purified monocytes and neutrophils to MSU stimulation

5.2.2.2 Identifying cells that produce pro-inflammatory cytokines after exposure to MSU crystals

5.2.3 Investigating activation of macrophages following exposure to MSU. 
5.2.4 Differential cytokine production by peritoneal leukocytes and peritoneal epithelial cells

\subsection{Results}

6.2.1 Monocytes differentiation in vivo

6.2.1.1 Mononuclear phagocyte morphology 175

6.2.1.2 Expression of the dendritic cell marker CD11c 178

6.2.1.3 Tracking monocyte differentiation through cell labelling 178

6.2.2 Functional changes in differentiating monocytes

6.2.2.1 Production of pro-inflammatory cytokines in response to MSU

6.2.3 Investigating phagocytic capacity

6.2.3.1 Expression of CD206

6.2.3.2 Functional phagocytic bead assay

6.2.3.3 Phagocytic capacity of differentiating monocytes

6.2.4 Investigating the effect of neutrophil uptake on monocyte responses

6.2.4.1 Monocyte phagocytosis of apoptotic cells

6.2.4.2 The uptake of apoptotic neutrophils leads to macrophage 201 quiescence

6.2.5 Monocyte-macrophages in the resolution of inflammation 
7.3 Monocytes in gouty inflammation $\quad 220$

7.4 Resolution of gouty inflammation 223

$\begin{array}{ll}7.5 \text { Neutrophils in gout } & 223\end{array}$

7.6 Inhibiting superoxide production as a therapy for gouty inflammation $\quad 227$

7.7 Dissecting the roles of inflammatory cells in gout 228

$\begin{array}{ll}7.8 \text { Future directions } & 229\end{array}$

$\begin{array}{ll}7.9 \text { Conclusion } & 231\end{array}$

$\begin{array}{ll}\text { Chapter 8. References } & 235\end{array}$

$\begin{array}{ll}\text { Appendix } & 253\end{array}$ 


\section{List of Figures}

\section{Chapter 1. General Introduction}

Figure 1.1 Monosodium urate crystals.

Figure 1.2 Acute and chronic gout. 6

Figure 1.3 Simplified uric acid production and excretion. 8

Figure 1.4 Schematic of urate handling in the kidney. 10

Figure 1.5 Steps catalysed by xanthine oxidase (XO) in the breakdown of 17 purines into uric acid.

Figure 1.6 The formation of reactive oxygen species is dependent on superoxide. 30

Figure 1.7. Equations outlining the reactions in the formation of ROS and the enzymes involved

Figure 1.8 A proposed model for the involvement of monocytes and macrophage in acute gout.

\section{Chapter 3. Neutrophil activation in MSU-induced inflammation}

Figure 3.1 The NADPH oxidase enzyme.

Figure 3.2 Current model of neutrophil activation in gout.

Figure 3.3 MSU stimulated production of superoxide by human neutrophils. $\quad 79$

Figure 3.4 The effect of different media conditions on background superoxide $\quad 80$ production by human neutrophils.

Figure 3.5 The effect of serum on the production of superoxide by human neutrophils.

Figure 3.6 The effect of non-autologous serum on the production of superoxide by human neutrophils.

Figure 3.7 The effect of MSU on neutrophil viability and cytokine production. 86

Figure 3.8 The effect of other leukocytes on MSU-stimulated neutrophil viability. 
Figure 3.9 The effect of soluble mediators derived from MSU-stimulation on $\quad 94$ neutrophil viability.

Figure 3.10 The effect of soluble mediators derived from MSU-stimulation on 96 neutrophil IL-8 production.

Figure 3.11 Histogram comparing the effect of soluble mediators and MSU on 99 neutrophil IL-8 production.

Figure 3.12 The effect of MSU-stimulated soluble mediators on neutrophil superoxide production.

Figure 3.13 Production of superoxide by MSU recruited neutrophils.

Figure 3.14 Neutrophil viability over the course of the acute gout response. 103

Figure 3.15 Proposed model of neutrophil activation in gout.

\section{Chapter 4. Inhibiting neutrophil inflammation using sesquiterpene dialdehydes}

Figure 4.1 Sesquiterpene compound structures

Figure 4.2 The effect of sesquiterpene compounds on neutrophil viability after 116 30 minutes.

Figure 4.3 The effect of sesquiterpene compounds on neutrophil apoptosis.

Figure 4.4 The effect of sesquiterpene compounds on neutrophil inflammation in vivo.

Figure 4.5 The effect of serum on superoxide production from HUVECs.

Figure 4.6 The effect of sesquiterpene compounds on superoxide production by HUVECs.

Figure 4.7 The effect of DMSO on MSU-stimulated cytokine production.

Figure 4.8 The effect of sesquiterpene compounds on MSU-stimulated cytokine production.

\section{Chapter 5. Macrophages in the initiation of acute gout}

Figure 5.1 Identification of cell types involved in the MSU crystal 
Figure 5.2 Morphology of cells expressing the myeloid antigens 7/4 and Gr-1.

Figure 5.3 MSU induces cellular infiltration and cytokine production in vivo. $\quad 138$

Figure 5.4 IL-6 production by cells infiltrating the peritoneum in response to MSU.

Figure 5.5 TNF $\alpha$ production by cells infiltrating the peritoneum in response to MSU.

Figure 5.6 Infiltrating leukocytes are unresponsive MSU.

Figure 5.7 Leukocytes infiltrating in response to thioglycollate are unresponsive MSU.

Figure 5.8 MSU-elicited monocytes and neutrophils are unresponsive MSU. $\quad 148$

Figure 5.9 Macrophages are the major producers of MSU-induced pro-inflammatory cytokines in lavaged cells of naïve mice.

Figure 5.10 Resident macrophages disappear from the peritoneal lavage following MSU administration.

Figure 5.11 MSU crystals become associated with cells as clumps in vivo.

Figure 5.12 Resident macrophages associate with MSU crystals in vitro.

Figure 5.13 Resident macrophages adhere to the epithelial membrane in response to MSU.

Figure 5.14 Resident macrophages are a source of pro-inflammatory cytokines in response to $\mathrm{MSU}$.

Figure 5.15 Depletion of resident macrophages inhibits MSU-induced neutrophil infiltration.

Figure 5.16 Depletion of resident macrophages inhibits MSU-induced cytokine production.

Figure 5.17 Differential production of pro-inflammatory cytokines by peritoneal leukocytes and membrane following MSU exposure.

Figure 5.18 Mesothelial cells produce MCP-1 in response to MSU. 


\section{Chapter 6. Profiling monocyte differentiation in acute MSU-induced inflammation}

Figure 6.1 Marker expression on monocytes over the course of an

MSU-induced acute inflammatory response.

Figure 6.2 Morphology of mononuclear phagocytes over the course of the

MSU-induced acute inflammatory response.

Figure 6.3 Cell width of mononuclear phagocytes over the course of the

MSU-induced acute inflammatory response

Figure 6.4 Expression of the dendritic cell marker CD11c over the course of an

MSU-induced acute inflammatory response.

Figure 6.5 PKH26 labelling of mononuclear phagocytes.

Figure 6.6 Marker expression on $\mathrm{PKH} 26^{+}$monocytes over the course of an

MSU-induced acute inflammatory response

Figure 6.7 F4/80 expression on differentiating monocytes over the course of an

MSU-induced acute inflammatory response

Figure 6.8 Responsiveness of peritoneal exudate cells to MSU restimulation

Figure 6.9 Stimulation of sorted macrophage populations ex vivo

Figure 6.10 In vivo restimulation with a second injection of MSU

Figure 6.11 Hematoxylin and Eosin staining of peritoneal tissue

Figure 6.12 Expression of the mannose receptor CD206 on $\mathrm{F} 4 / 80^{+}$cells over the course of an MSU-induced acute inflammatory response.

Figure 6.13 Optimisation of bead concentration for use in the phagocytosis assay 195

Figure 6.14 Phagocytosis of fluorescent beads by monocyte-macrophages 196

Figure 6.15 Enumeration of beads taken up by monocyte-macrophages

Figure 6.16 Phagocytic capacity of macrophages from mice $72 \mathrm{~h}$ after in vivo MSU treatment

Figure 6.17 Peroxidase staining of peritoneal exudate cells

Figure 6.18 Percentage of myeloperoxidase positive $\left(\mathrm{MPO}^{+}\right)$monocyte- 
Figure 6.19 IL-1 $\beta$ production from peritoneal leukocytes following neutrophil 206 uptake

Figure 6.20 TGF $\beta$ levels in the peritoneum over the course of an MSU-induced 208 inflammatory response.

Figure 6.21 Summary of the phenotypic and functional changes during the 209 differentiation of monocytes to macrophages over the course of MSU-induced inflammation.

\section{Chapter 7. General Discussion}

Figure 7.1 Model of acute gouty inflammation 


\section{List of Tables}

Table 1.1. Expression levels of selected markers on resident and inflammatory monocytes expression markers.

Table 4.1 $\mathrm{IC}_{50}$ values of sesquiterpene compounds based on PMA- and MSU-stimulated superoxide production by neutrophils.

Table 4.2 A comparison of sesquiterpene $\mathrm{IC}_{50}$ values for neutrophil superoxide production with sesquiterpene $\mathrm{TC}_{50}$ values.

Table 4.3 Comparison of $\mathrm{IC}_{50}$ values of the sesquiterpene compounds from HUVEC superoxide production, with $\mathrm{IC}_{50}$ PMA- and MSU-stimulated neutrophil production.

Table 5.1 Cell composition of the peritoneum during an inflammatory response to MSU crystals.

Table 6.1 Differential cell counts in peritoneal cells from naïve mice and from mice treated with MSU i.p. for $48 \mathrm{~h}$. 


\section{List of Abbreviations}

\begin{tabular}{|c|c|}
\hline ADP & Adenosine diphosphate \\
\hline AMP & Adenosine monophosphate \\
\hline ASC & $\begin{array}{l}\text { Apoptosis-associated speck-like protein containing a caspase recruitment } \\
\text { domain }\end{array}$ \\
\hline ATP & Adenosine triphosphate \\
\hline bFGF & Bovine fibroblast growth factor \\
\hline BSA & Bovine serum albumin \\
\hline CBA & Cytokine bead array \\
\hline Clodronate & Dichloromethylenediphosphonic acid disodium salt \\
\hline $\mathrm{CNS}$ & Central nervous system \\
\hline $\mathrm{ddH}_{2} \mathrm{O}$ & Double-distilled (MilliQ) water \\
\hline DIC & Differential interference contrast \\
\hline DMSO & Dimethyl sulfoxide \\
\hline D-PBS & Dulbecco's Phosphate-Buffered Saline \\
\hline DPI & Diphenyleneiodonium chloride \\
\hline EGF & Epidermal growth factor \\
\hline FACS & Fluorescence activated cell sorter \\
\hline FBS & Fetal bovine serum \\
\hline FGF & Fibroblast growth factor \\
\hline fMLP & formyl-methionyl-leucyl-phenylalanine \\
\hline G-CSF & Granulocyte colony stimulating factor \\
\hline GLUT9 & Glucose transporter 9 \\
\hline GM-CSF & Granulocyte macrophage colony stimulating factor \\
\hline HBSS & Hank's balanced salt solution \\
\hline HEPES & 4-(2-hydroxyethyl)-1-piperazineethanesulfonic acid \\
\hline HuSer & Human serum \\
\hline HUVEC & Human umbilical vein endothelial cells \\
\hline IFN $\gamma$ & Interferon gamma \\
\hline IL-1 $\beta$ & Interleukin-1 beta \\
\hline
\end{tabular}


$\mathrm{KC} \quad$ Kertinocyte-derived chemokine

L. monocyto- Listeria monocytogenes

$$
\text { genes }
$$

LPS Lipopolysaccharide

M199 Medium 199

MCP-1 Monocyte chemoattractant protein-1

M-CSF Macrophage colony stimulating factor

MIP Macrophage inhibitory protein

MMP-1 Matrix metalloproteinase-1

MRP4 Multidrug resistence protein 4

MSU Monosodium urate

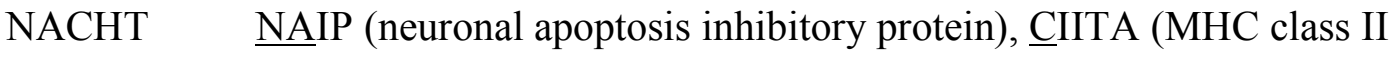
transcription activator), HET-E (incompatibility locus protein from

Podospora anserina) and TP1 (telomerase-associated protein)

NADPH Nicotinamide adenine dinucleotide phosphate (reduced)

NALP3 NACHT-leucine rich repeat-pyrin domain-containing protein-3

(NALP)-3 (cryopyrin)

NLR Nod-like receptors

NOX Nicotinamide adenine dinucleotide phosphate oxidase

OAT Organic anion transporter

PBMC Peripheral blood mononuclear cells

PBS Phosphate buffered saline

PFA Paraformaldehyde

PSGL1 P-selectin glycoprotein ligand 1

Phox Phagocytic oxidase

PI Propidium iodide

PMA Phorbol 12-myristate 13-acetate

PMN Polymorphonuclear cells

PMS 1-methoxy-5-methylphenazinium methylsulfate

RANKL Receptor activator of nuclear factor kappa B

RBC Red blood cells 
TG2 Transglutaminase-2

TBS Tris buffered saline

TNF $\alpha \quad$ Tumor necrosis factor alpha

TLR Toll-like Receptor

TREM Triggering receptor expressed on myeloid cells

SLC22A12 Solute carrier 22 (organic anion/cation transporter), member 12

URAT-1 Urate transporter-1

WBC White blood cells

WST-1 2-1[2-(4-Iodophenyl)-3-(4-nitrophenyl)-5-(2,4-Disulfophenyl)-2Htetrazolium, monosodium salt] 
Chapter 1

General Introduction 


\section{Chapter 1 \\ General Introduction}

\subsection{A history of gouty disease}

Gout is a form of arthritis with a documented history spanning several thousands of years. Observed by the ancient Egyptian medical practitioner Imhotep as early as 2640BCE (Schwartz, 2006), it was also described several centuries later by the Greek physician Hypocrates (400BCE), the father of modern medicine, who referred to it as podagara, or "the unwalkable disease" (Nuki and Simkin, 2006).

The pre-eminence of gout in historical texts is due not only to its enduring incidence but also to its prevalence among the wealthy and those of high society. Several kings and notable historical figures are included in the cadre of sufferers: from Alexander the great, to Henry VIII, Christopher Columbus, Leonardo DaVinci, Isaac Newton, Benjamin Franklin (Johnson and Rideout, 2004). The pedigree of sufferers often led to the erroneous notion that gout was a natural and unavoidable affliction of high breeding that correlated with a person's level of intelligence (Porter and Rousseau, 1998). Despite this belief, it did not escape observation that it was the lifestyle rather than the pedigree of the rich that was the likely cause for the disease. In fact, it was often said that gout was a punishment for "old men ... after passing the best part of their life in ease and comfort, indulging freely in high living, wine and other generous drinks." (Sydenham, 1683)

In the present day, gout is no longer restricted to the circles of affluent society. In fact, the striking increase in the number of gout sufferers across the full range of socioeconomic groups highlights the emergence of gout as a common disease in western society. Gout prevalence in the US increased from 2.9/1000 in 1990 to 5.2/1000 in 1999 (Wallace et al., 2004). Another based on research in England during the same period, placed prevalence at 9.5/1000 (Harris et al., 1995). An even more impressive rise was observed in New Zealanders of European descent, which increased from 3.1/1000 in 
1958 to 29/1000 in 1992 (Lennane et al., 1960, Klemp et al., 1997). These statistics reflect a global trend clearly indicating that gout is on the rise worldwide.

The alarming acceleration of gout incidence has emphasised the need for a deeper understanding of gout as a disease. The following pages aim to provide an overview of what is currently known about gout. This review will describe the cause, symptoms and stages of gout; discuss the known risk factors and management practices; and outline key background information on the cellular events known to be involved in gouty inflammation. Finally, the research questions of this thesis will be presented. These questions will address some of the important unknown aspects of cellular inflammation in gout. Ultimately, it is the goal of this thesis to provide a significant advance in the understanding of the cellular events that characterise gout inflammation.

\subsection{Monosodium urate crystals}

Although needle-like crystals were observed in gout afflicted joints as early as the $17^{\text {th }}$ century (Gibson et al., 1984), it was not until the 1960s that these crystals, called monosodium urate or MSU, were experimentally confirmed as the inducing agent in a gout attack by Faires and $\mathrm{M}^{\mathrm{c}}$ Carty. Using MSU they had prepared synthetically, they injected crystals into the synovial space of their own knees. The result was a full-blown gout attack (Faires and McCarty, 1962).

MSU crystals are the biological form of crystallised uric acid. They are needle-like in appearance and have the ability to split incident light; a quality called birefringence that is observed microscopically using plane polarised light (Fig. 1.1). MSU crystals can be identified in synovial fluid extracted from joints affected by gout even after the gout attack has resolved and there is no clinical evidence of inflammation (Pascual, 1991, Pascual and Jovani, 1995). Thus, once uric acid has precipitated in the joint it takes a long time for MSU crystals to be redissolved by the body as soluble uric acid. 

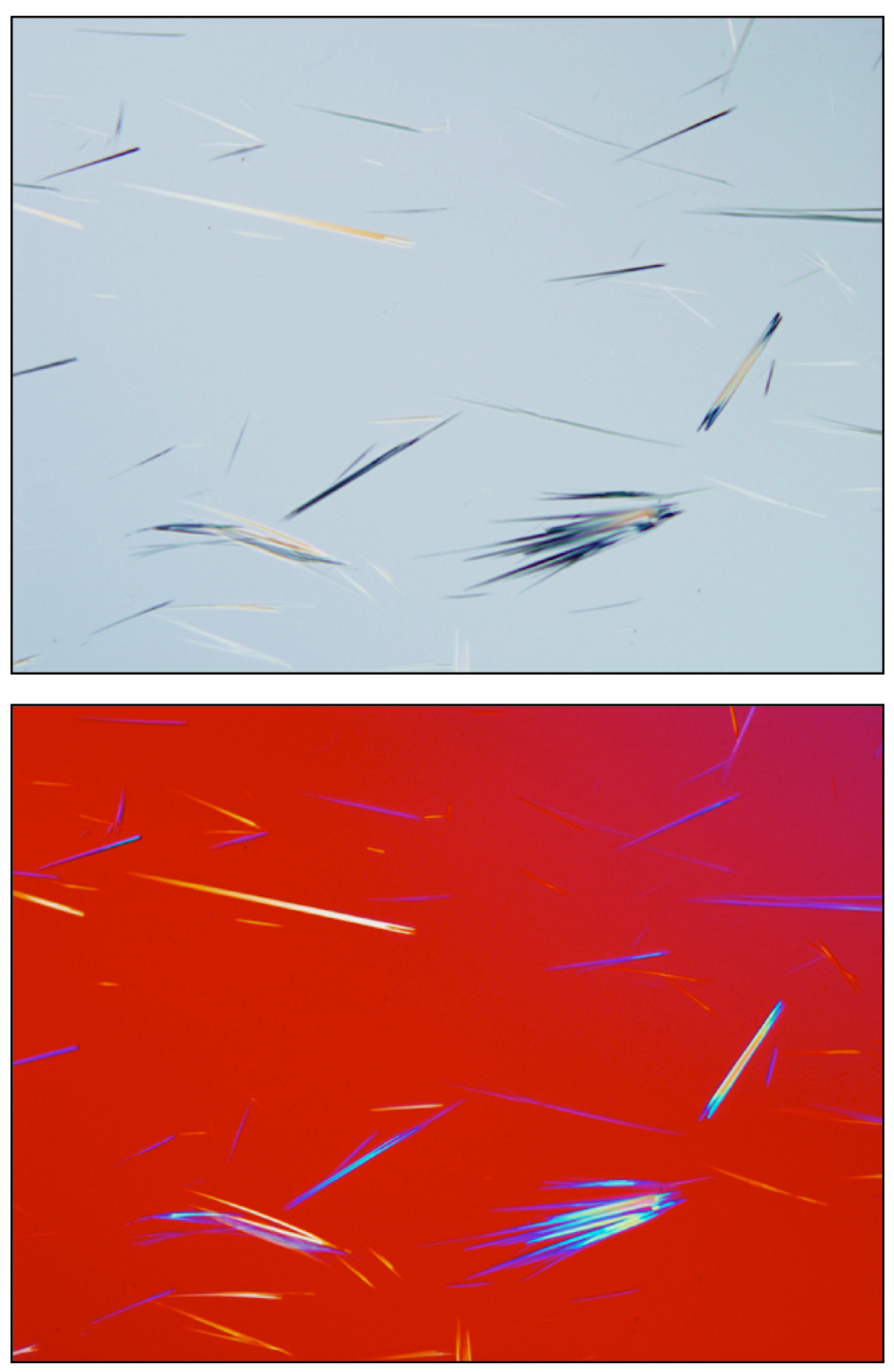

Figure 1.1. Monosodium urate crystals. Monosodium urate crystals (MSU) as visualised under a microscope in normal (top) and polarised (bottom) light fields. 


\subsection{Acute gouty arthritis}

In 1683, the physician Thomas Sydenham provided what is now the classic description of gout. Speaking from his own experience, he described:

"The victim goes to bed and sleeps in good health. About two o'clock in the morning, he is awakened by a severe pain in the great toe; more rarely in the heel, ankle or instep. This pain is like that of a dislocation, and yet the parts feel as if cold water were poured over them. Then follows chills and shiver and a little fever. The pain, which at first moderate, becomes more intense. After a time this comes to full height, accommodating itself to the bones and ligaments of the tarsus and metatarsus. Now it is a violent stretching and tearing of the ligaments - now it is a gnawing pain and now a pressure and tightening. So exquisite and lively meanwhile is the feeling of the part affected, that it cannot bear the weight of bedclothes nor the jar of a person walking in the room." (Sydenham, 1683)

This description underpins the major aspects of acute gout: that it occurs suddenly, in one or more joints, and is extremely painful. During a gouty episode the affected joint exhibits all of the classic symptoms of inflammation (Fig. 1.2A). The joint swells and reddens as vascular permeability increases, allowing fluids to drain into the area. This inflammatory reaction is accompanied by intense pain and heat in the affected joint. The symptoms of oedema, rubor, heat and pain, will continue for several days, marking the rich influx of neutrophils - a key cell in gout - along with monocytes and other proinflammatory leukocytes into the area. Even in the absence of therapy, the inflammatory symptoms will subside within 7-10 days (Poor and Mituszova, 2003). The abrupt onset and spontaneous resolution of gout are characteristic of this acute response. In terms of frequency of acute attacks, 60\% of individuals who experience their first gout attack experience a second attack within the space of a year (Ferraz and O'Brien, 1995). 

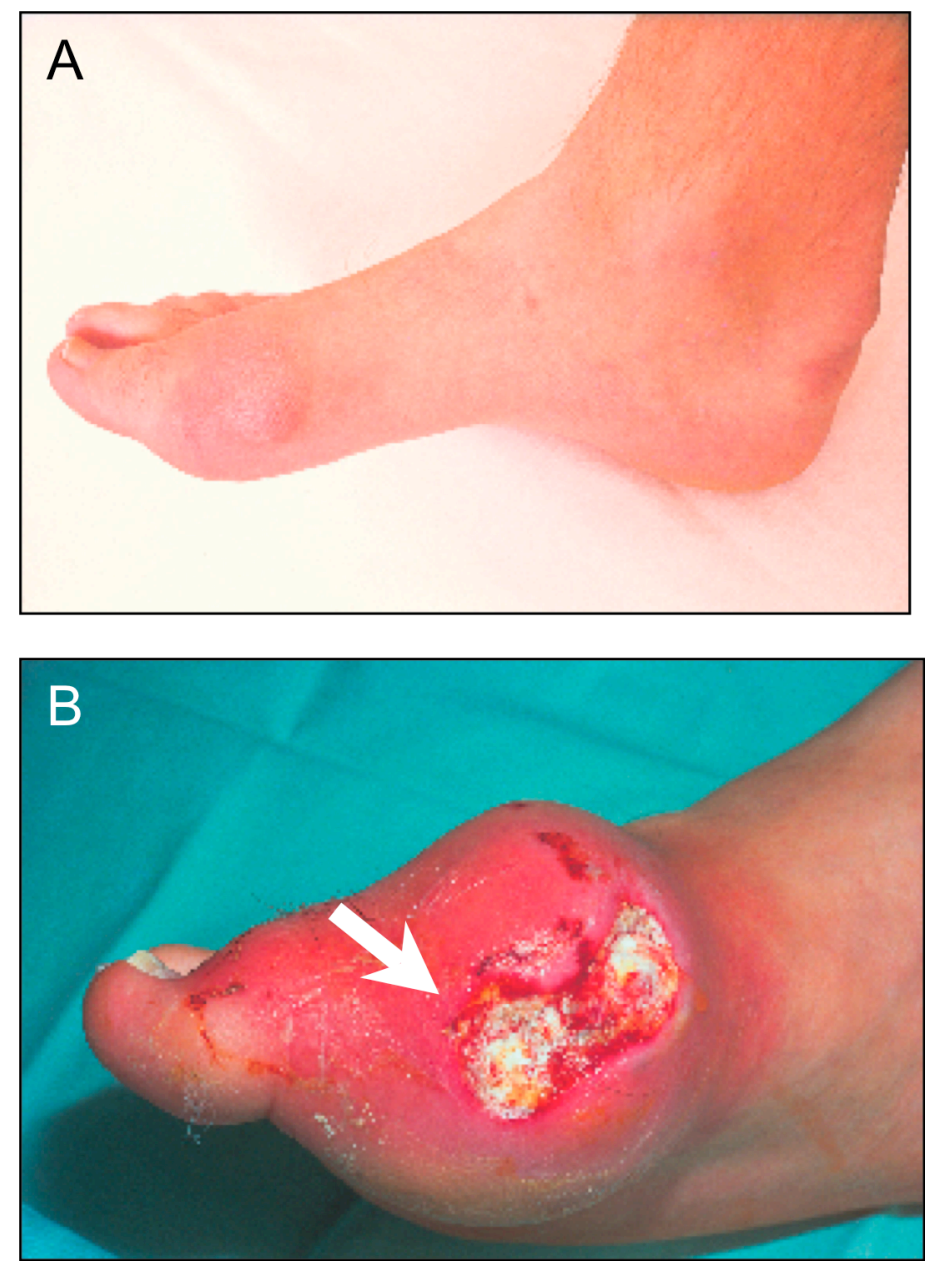

Figure 1.2. Acute and chronic gout. Pictures of joints from patients with (A) acute gout and (B) chronic tophaceous gout. The white arrow indicates the MSU crystals of a tophus that has ruptured through the skin and caused ulceration. Pictures used with permission from PHARMAC, NZ. 


\subsection{Chronic gout}

Sufferers of acute gout may experience periodical attacks for several years over which time these attacks can become more frequent and severe. This increase in intensity can be accompanied by the formation of lumps or nodules, called tophi: collections of MSU crystals and immune cells that have accumulated in the joints or soft tissue (Fig. 1.2B). The formation of tophi around the joints leads to polyarticular bone erosions and deformations (Nakayama et al., 1984). The development of chronic gout is often debilitating, with some individuals never experiencing complete freedom from ongoing pain. In addition, individuals will continue to experience periodic attacks of inflammation, known as gout flare.

\subsection{Uric acid}

The greatest predictor of gout risk and development is the concentration of uric acid in the serum. Uric acid levels commonly fall into the range of $2.4-6.0 \mathrm{mg} / \mathrm{dL}$ in women and $3.4-7.0 \mathrm{mg} / \mathrm{dL}$ in men (Dincer et al., 2002). Clinical hyperuricemia is diagnosed when serum uric acid exceeds $7 \mathrm{mg} / \mathrm{dL}$. At this elevated level the uric acid load approaches the solubility limit, moving individuals into a high risk category of contracting gout (Campion et al., 1987).

Uric acid is routinely generated in the body as a breakdown product of purines (Poor and Mituszova, 2003). Purines are an essential building block in many essential proteins, enzymes, structures and energy carrying molecules including DNA, NAD, NADP and ATP. The purine requirements of the body can mostly be synthesised endogenously and maintained through purine recycling and salvage pathways (Murray, 1971). Anything that increases the rate of purine synthesis or breakdown, such as surgery, increased cell turnover, cell damage and necrosis, or high intake of fructose or alcohol (Section 1.7.2), potentially affects serum uric acid level. Purines can also come from exogenous sources through the intake of purine rich foods such as seafood and meat (Section 1.7.2). A simplified version of the causes of uric acid generation is shown in Fig. 1.3. 
nucleotide synthesis

or metabolism
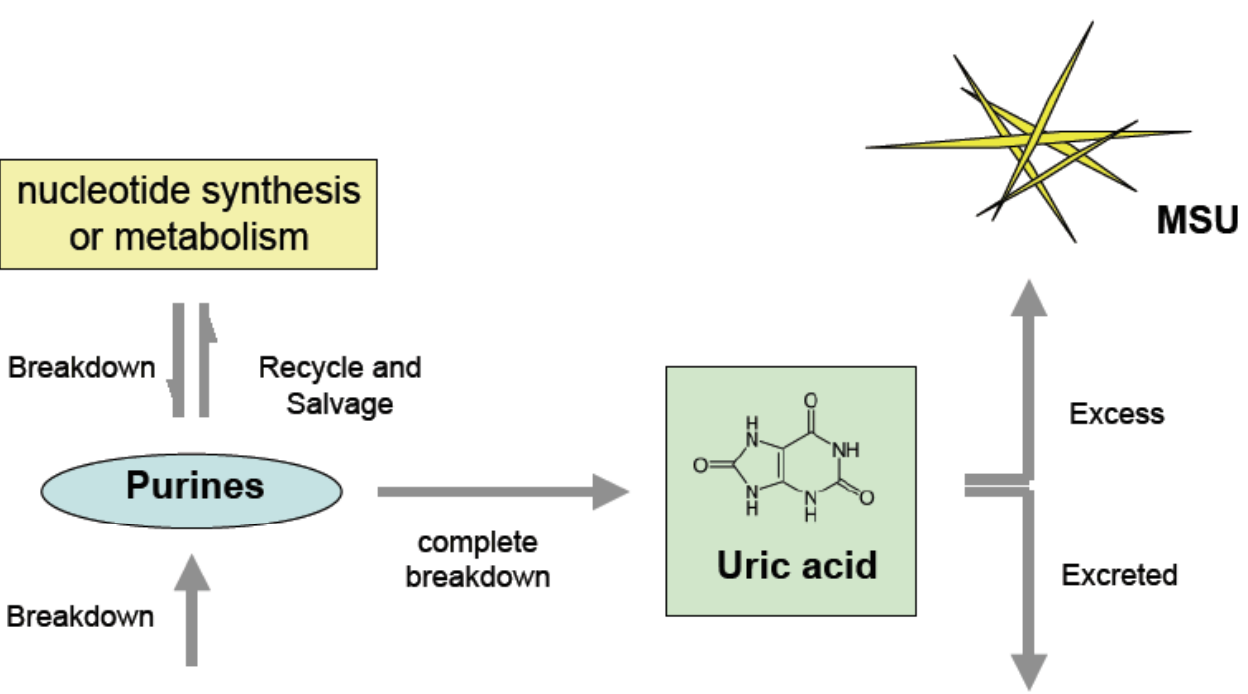

diet
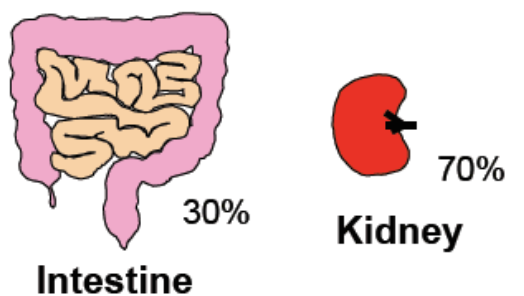

Kidney

Figure 1.3. Simplified uric acid production and excretion. Both dietary and metabolic sources contribute to the overall purine pool within the body. Excess and unsalvageable purines are broken down into uric acid, which is excreted through the kidney (70\%) and intestine (30\%). When the uric acid load becomes too high, MSU crystals may begin to form in the joint. Adapted from Roddy, Zhang et al. 2007. 
In humans, renal excretion is the main process for uric acid extinction, accounting for approximately $70 \%$ of uric acid excretion. The remainder of urate is excreted through the intestine into the faeces (Fig. 1.3). Together these two paths are entirely responsible for controlling uric acid excretion in humans (Poor and Mituszova, 2003).

Kidney urate handling is a key contributor to high serum uric acid (Terkeltaub et al., 2006). In the kidney, uric acid handling is a multi-step process that results in the excretion of only $10 \%$ of the uric acid filtered through the kidney. Urate in the blood is filtered at the glomeruli and is almost completely reabsorbed into the bloodstream in the upper section of the kidney tubules. Urate is then re-secreted into the tubules followed by post-secretory re-absorption before urate is finally excreted in the urine (Fig. 1.4). This multi-step process is controlled by a number of transport proteins (e.g. URAT-1, OAT-1, OAT-3 and MRP4) suggesting that there are a number of points at which susceptibility to hyperuricemia due to kidney function may be due to differences in one or more of these proteins leading to overly efficient re-absorption of uric acid into the blood, or under-efficient re-secretion into the kidney tubules: under-excretion (Hediger et al., 2005, Taniguchi and Kamatani, 2008)

\subsection{Loss of uricase}

Unlike humans, most other mammals have an additional enzyme for regulating serum urate known as uric acid oxidase, or uricase. Uricase efficiently converts uric acid to the more soluble allantoin, which is readily passed out in the urine. The efficacy of this enzyme is highlighted by the fact that mammals with functional uricase do not contract gout (Johnson and Rideout, 2004). The uricase gene still exists within the human genome, however, the gene for human uricase is inactive due to a mutation that occurred in humans during the Miocene era, around 10 to 22 million years ago (Wu et al., 1989, Wu et al., 1992). This loss of uricase activity explains the large difference in serum uric acid levels observed in uricase sufficient mammals, which maintain a low serum urate level of less than 1mg/dL (Wu et al., 1992). 


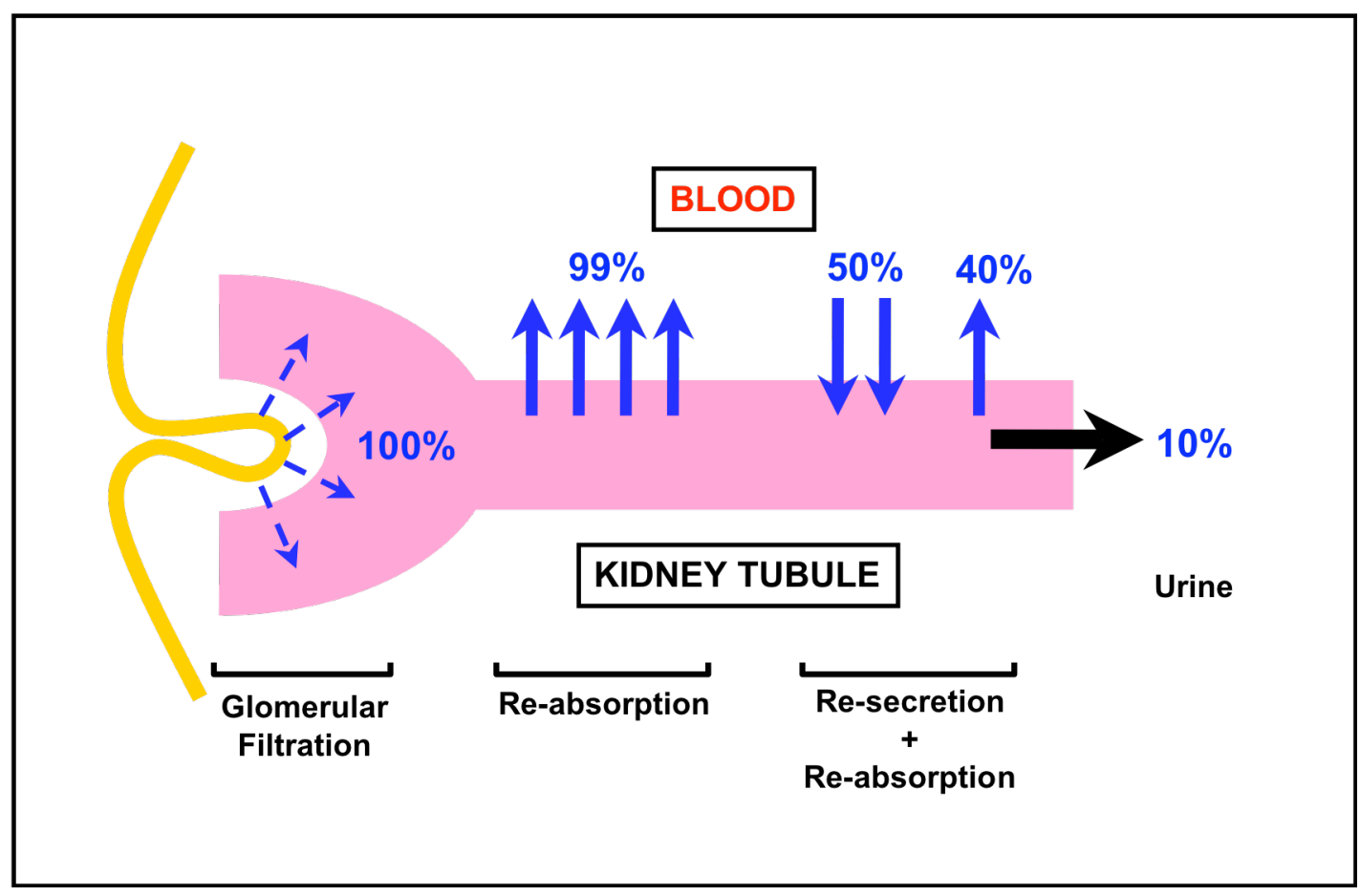

Figure 1.4. Schematic of urate handling in the kidney. Approximately $99 \%$ of the filtered urate (blue arrows) is reabsorbed back into the blood stream by transport proteins that line the lumen of the tubule. Urate is then re-secreted and re-absorbed by further transport proteins involved in the exchanging of various ions resulting in a net $90 \%$ retention of urate. The other $10 \%$ is excreted through the urine (black arrow). Blue arrows show the movement of urate across tissues. Adapted from Poor et al. 2003. 
There are other mutations of the uricase gene in other higher primates, independent of the human mutation, implying that the lack of uricase and a heightened serum uric confers some selective advantage (Wu et al., 1992, Watanabe et al., 2002). One possible benefit of a loss in uricase during that period may have been an advantageous increase in blood pressure. It is thought that during the Miocene era, when the mutations in uricase arose, primates would have maintained a largely vegetarian diet, which was low in sodium, resulting in low blood pressure. Low blood pressure is problematic when spending a large amount of time "standing" erect, as blood flow to the brain is less efficient. Rat studies have shown that a low sodium diet causes low blood pressure, however an increase in uric acid levels by inhibiting rat uricase caused an increased in blood pressure to normal levels (Mazzali et al., 2001). A small human study has also shown that therapy aimed at lowering serum uric acid also lowered blood pressure (Feig and Johnson, 2007). It is ironic that this evolutionary advantage may have become disadvantageous in the current environment of a modern western diet.

Another possible advantage of a loss in uricase was to harness the function of uric acid as an antioxidant. Uric acid is the most abundant antioxidant present in the body, and as such, is likely to play an important role in eliminating errant reactive oxygen species in the circulation and tissues (Ames et al., 1981 \& Hochstein, 1981, Becker, 1993). For example, it has been hypothesized that this antioxidant effect is important in protection from the oxidative damage in the central nervous system (CNS) caused by peroxynitrite. The administration of uric acid to mice with experimental allergic encephalomyelitis, a disease used to model human multiple sclerosis, has been shown to delay and even reverse clinical symptoms of the disease (Hooper et al., 1998). Meanwhile, patients with Parkinson's disease have demonstrated a lower plasma uric acid level than healthy individuals, suggesting that the loss of antioxidant protection is involved with the progress of CNS damage (Annanmaki et al., 2007). The antioxidant effects of uric acid may be particularly important in humans, since humans lost the ability to sythesize ascorbate, an important antioxidant in most other organisms (Proctor, 1970). Therefore, 
it is highly likely that heightened uric acid levels in humans serves as a general protective mechanism against the generation of oxidative damage under both homeostatic and inflammatory conditions.

\subsection{Gout risk factors}

Even though the precipitation of MSU crystals is predicted by hyperuricemia, only $25 \%$ of hyperuricemic individuals develop gout (Campion et al., 1987). Some individuals can be in a state of asymptomatic hyperuricemia for several years before suffering a gout attack, while in the vast majority high uric acid will never precipitate into an attack. MSU crystals can also be present within the joint between attacks without elaborating an inflammatory response (Pascual et al., 1999). The reasons for these observed phenomena are currently unknown and demonstrates that there are still a number of mysteries surrounding the onset of gout that have yet to be unravelled.

\subsubsection{Gender}

A prime risk factor in gout is gender. Gout is the most common form of inflammatory arthritis in men over 40 (Roubenoff, 1990). In fact, gout is heavily a predominantly male disease where prevalence in men is typically four-fold that observed in women (Hall et al., 1967). It is currently believed that women are protected from gout due to the uricosuric effects of estrogen and consistent with this idea is the observation that it is rare for women to suffer gout prior to menopause (Nicholls et al., 1973). Testosterone is also known to increase the expression of URAT1, a key protein in urate reabsorption in the kidney, further implicating sex hormones as the cause of gender differences (Li et al., 2004).

\subsubsection{Diet}

Dietary purine intake is a significant contributor to serum uric acid levels (Griebsch and Zollner, 1974, Coe et al., 1976). The intake of high purine foods is often sufficient to induce the onset of a gout attack in hyperuricemic individuals, making diet a major risk factor in gout. Purine rich foods include shellfish (mussels, oysters, clams), seafood (sardines, anchovy, trout), red meat, offal foods (liver, heart, tongue, kidney, brain), 
asparagus, mushrooms and beer. In particular, prospective studies have shown that individuals with high meat and/or seafood intake have elevated serum urate levels and are at the highest risk of developing gout (Choi et al., 2004b, Choi et al., 2005).

Other foods associated with increased risk, including fructose (Choi and Curhan, 2008, Choi et al., 2008) and some alcoholic beverages (Choi et al., 2004a, Choi and Curhan, 2004), increase serum uric acid indirectly. For example, fructose, which is a common sweetener in soft drinks and fruit juices, is a major contributor to uric acid levels by interfering with ATP recycling, causing ATP to be converted to AMP and subsequently degraded to uric acid rather than being recycled from ADP back to ATP (Fox and Kelley, 1972, Fox et al., 1987). In addition, this reduction in ATP stores causes the body to increase the rate of purine synthesis in the liver to replenish the degraded ATP, further raising the pool of purines available for degradation to uric acid (Raivio et al., 1975). Alcohol also leads to an increase in purines by increasing ATP turnover during the metabolism of ethanol (Faller and Fox, 1982, Puig and Fox, 1984), the increased turnover leading to a similar elevated purine breakdown as observed in fructose consumption.

\subsubsection{Genetic and race-related risk}

As mentioned previously, genetics also play a part in gout risk. Since intrinsic overproduction of purines is rare, increased genetic risks are likely to be due to differences in renal excretion. Recently, genetic studies have identified two genes and their respective proteins as having a strong influence on the regulation of serum urate: URAT1 and GLUT9. Both proteins are involved in urate reabsorption following glomerular filtration. Polymorphisms in SLC22A12, the gene that encodes URAT1, have been shown to be associated with both hyperuricemia and hypouricemia (Ichida et al., 2004, Shima et al., 2006, Vazquez-Mellado et al., 2007), while a particular allele of GLUT9 has been associated with a reduction in serum urate in Italian, UK, German and Croatian populations (Li et al., 2007, Stark et al., 2008). These genes are a good starting point for exploring racial and genetic susceptibilities, and since uric acid handling in the kidney is a multi-step process, other genetic susceptibilities are likely to be revealed. 
The over-representation of gout in certain racial groups implies that there may be genetic causes to explain the high prevalence. As a case example, Maori have more than twice the incidence of gout than non-Maori in New Zealand (Klemp et al., 1997). It has not yet been ruled out that this high incidence is due to factors other than genetics. Gout, for instance, had been notably absent within Maori prior to 1880 (Lennane et al., 1960). Yet after the conversion of the Maori diet from a high vegetable and carbohydrate diet to a high meat and high fat diet, gout quickly emerged in extreme prevalence (Lennane et al., 1960), where now approximately 10\% of adult Maori males contract gout (Klemp et al., 1997).

\subsubsection{An aging population}

The risk of contracting gout increases with age (Wallace et al., 2004). Increased longevity in industrialised nations may therefore account for some of the increase in gout prevalence that has been observed (Saag and Choi, 2006). This is influenced by a decrease in renal function with advancing age and further complicated by some medications commonly used by the elderly that have diuretic side effects that are also capable of raising serum urate levels (De Leonardis et al., 2007). The treatment of gout within the elderly is often complicated by renal failure, intolerance to certain treatments and the presence of other health conditions that can limit the medications for gout (De Leonardis et al., 2007).

\subsection{Co-morbid diseases}

If the need for increased blood pressure was indeed a contributing factor in the loss of uricase in humans, it is unfortunate that the modern western diet now drives hypertension to almost epidemic proportions. However, it does provide an explanation for the existence of hyperuricemia and hypertension as co-morbid diseases (Messerli et al., 1980). In fact, several studies have linked hyperuricemia not only to hypertension, but also to other metabolic syndrome-based disorders including cardiovascular disease (Puig and Ruilope, 1999), making it an associated risk factor for a wider collection of globally increasing diseases. 


\subsection{Treatment and management of gout}

There are three main approaches in the management of gout. The first is based on a lifestyle approach, focused on controlling serum uric acid by moderating food intake. The second involves the use of drugs aimed to lower serum urate and tends to be a longterm regimen. The third uses non-steroidal anti-inflammatory drugs to alleviate the symptoms of inflammation associated with a gout attack.

\subsubsection{Diet}

There are a number of studies showing that the intake of certain foods are associated with heightened serum urate levels. Limiting the intake of purine-rich foods and uric acid promoting substances such as alcohol are recommended to prevent raising uric acid levels and precipitating gout attacks (Choi et al., 2004b, Choi et al., 2004a). Conversely, there are a number of dietary items associated with small reductions in serum uric acid and lower gout risk. Low fat-dairy (Choi et al., 2004b), cherries (Jacob et al., 2003), vitamin C (Huang et al., 2005, Gao et al., 2008) and long term coffee drinking (Choi and Curhan, 2007, Choi et al., 2007) are examples, although the cause of the urate lowering effects of these treatments are currently unknown.

Research has shown that being overweight increases the risk of gout (Choi et al., 2005). A small study has also shown that weight loss can improve gout symptoms. In this research weight loss of as little as 5 to $8 \mathrm{~kg}$ in obese gout sufferers improved serum urate levels by $11 \%$ and reduced the average attacks from 2.1 to 0.6 episodes per month within the groups of patients observed (Dessein et al., 2000). 


\subsubsection{Anti inflammatory drugs}

Gout attacks are intensely painful. For this reason, anti-inflammatories are used to alleviate the symptoms of gout attacks. This includes the use of glucocorticoids, but more commonly involves the use of non-steroidal anti-inflammatory drugs (NSAIDs) including Indomethacin, Naproxen and Ibuprofen (Jordan et al., 2007). NSAIDs can be used as a prophylaxis to disease or to alleviate the symptoms of inflammation during or immediately before the onset of an attack.

Colchicine is also used for the treatment of gout. It is the active component of the plant Autumn crocus, which has been used for several centuries as a remedy for gout prior to colchicine isolation in 1820 (Ahern et al., 1987). Colchicine can be used to alleviate the symptoms of an attack or as a low dose prophylactic regime. It has a number of known therapeutic activities including the inhibition of microtubule polymerisation that contributes to the inhibition of neutrophil migration and activation (Sternlicht and Ringel, 1979, Chia et al., 2008). In the past, the use of colchicine was often controversial due to its reputation as a potent poison. It has a narrow therapeutic window as a number of toxic side effects can be observed during treatment, such as diarrhoea, gastrointestinal damage, fever, vomiting and in a few extreme cases, death (Bonnel et al., 2002, Sussman et al., 2004). Since colchicine has the potential for these side effects it is not well tolerated in some individuals that are already physiologically compromised such as the elderly, or patients with renal failure.

\subsubsection{Uric acid lowering drugs}

The lowering of serum uric acid can also be achieved through the use of drugs that work by a number of mechanisms to lower serum urate, acting as a prophylaxis to gout attacks. These drugs accomplish their effect by either lowering the production of uric acid (uricostatic drugs) or by increasing renal excretion (uricosuric drugs). Allopurinol is a commonly prescribed uricostatic pro drug that is converted in the liver to the active metabolite oxypurinol (Becker et al., 2005). Oxypurinol prevents uric acid from being generated by inhibiting xanthine oxidase, the enzyme responsible for the last 


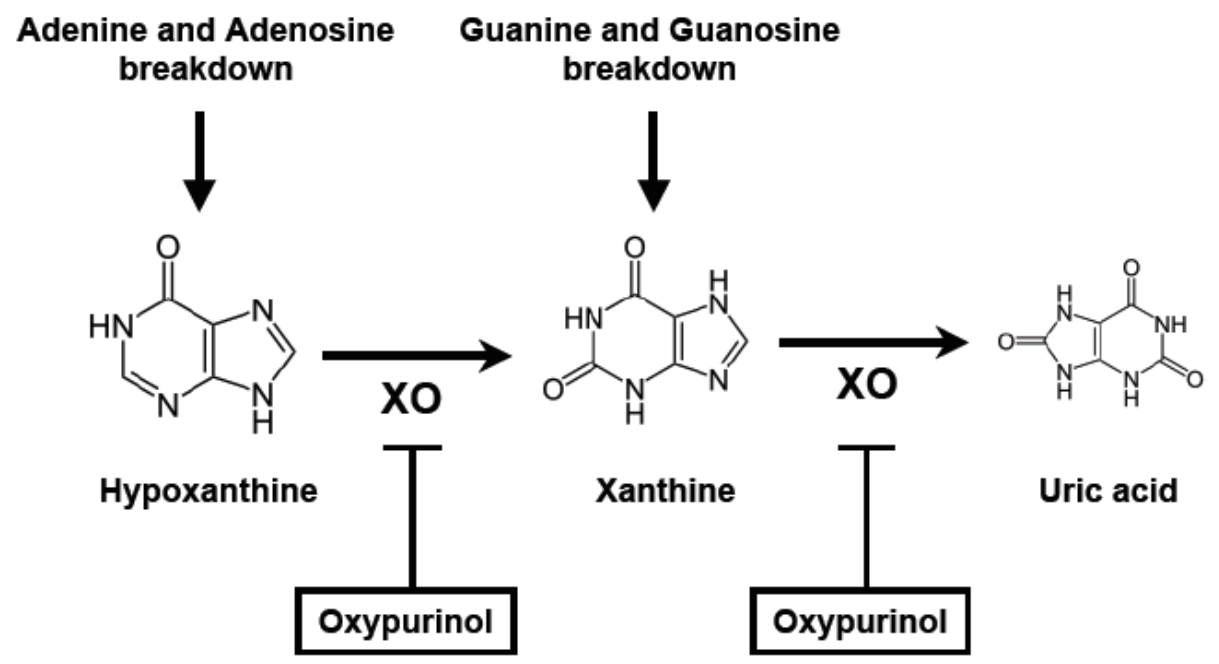

Figure 1.5. Steps catalysed by xanthine oxidase (XO) in the breakdown of purines into uric acid. The figure shows the steps that are inhibited by oxypurinol. (Adapted from Pacher et al., 2006). 
step in conversion of purines from hypoxanthine and xanthine to uric acid (Fig. 1.5). Febuxostat and Y-700 are additional examples of xanthine oxidase inhibitors (Bomalaski and Clark, 2004, Becker et al., 2005). Uricosuric drugs function by preventing reabsorption of uric acid in the kidney so that more uric acid gets excreted. Examples of these are probenecid, which inhibits the reabsorption of uric acid by competitive inhibition of the OAT urate transporter protein in proximal tubules in the kidney (Sweet et al., 2003); and benzbromarone, which inhibits URAT-1 mediated reabsorption of uric acid in tubules (Enomoto et al., 2002).

\subsubsection{Limitations of current therapies}

Despite the usefulness of these therapies, they are not without their limitations. For instance, the use of NSAIDs is often associated with side effects such as gastric ulcers (Conaghan and Day, 1994); some individuals with poor renal function cannot tolerate some medications (Mikuls et al., 2004); and the need for daily dosing of uricosuric drugs often leads to low compliance (Sarawate et al., 2006). In addition, gout often goes misdiagnosed or poorly managed. Thus, a deeper understanding of gouty disease may provide insights into new and more effective ways to identify and treat gout. It may suggest ways in which at risk individuals can be more easily identified and protected from gout attack, or it may identify the cell types that must be targeted in order to mitigate the inflammatory response or speed resolution. Indeed, there are many questions on the "bare bones" cellular level that may be key in revealing the important cellular inflammatory events that can be targeted for an improved clinical benefit.

\subsection{Cellular inflammation in acute gout inflammation}

In order for gout to be initiated, cells have to recognise and respond to MSU crystals, and induce inflammation. The nature of that response depends on the cell types recognising those crystals, and on the cells subsequently recruited to the site of inflammation. Some of the important cells and cellular mediators in gout have been 
identified, but much remains to be answered. This section summarises what is currently known about the cellular responses in gout inflammation providing the backdrop for the research that was undertaken in this thesis.

\subsubsection{Initiation of gout inflammation}

MSU initiates the activation of inflammatory cells in gout. When MSU forms within a joint, the local cells are activated to initiate an inflammatory response. Mast cells, monocytes, and synoviocytes are thought to contribute by producing inflammatory molecules including IL-1 $\beta$, TNF $\alpha$, IL-8, IL-6 and S100 proteins (Di Giovine et al., 1987, Guerne et al., 1989, di Giovine et al., 1991, Getting et al., 1997, Ryckman et al., 2003a). Proteins already present in the serum are also thought to be involved in activating inflammatory cells; for instance, complement proteins are thought to be activated at the surface of MSU crystals (Russell et al., 1982). Collectively these inflammatory mediators facilitate the recruitment of leukocytes via the upregulation of adhesion molecules on epithelial cells and the cognate adhesion molecules on responding leukocytes, enabling the recruitment of blood leukocytes into the joint space. Once neutrophils invade the joint, they are able to interact with and phagocytose MSU crystals to produce IL-8 and S100 proteins, which further augment leukocyte recruitment during the pro-inflammatory stage of the inflammatory response (Ryckman et al., 2004). Contact of neutrophils with MSU has also been shown to trigger the production of reactive oxygen species that contribute to the destructive inflammatory environment in gout (Abramson et al., 1982).

\subsubsection{The necessity of MSU recognition by immune cells as a "danger signal"}

MSU is a non-infectious agent and so induces a sterile inflammatory response. As it is a sterile response it may be difficult to understand why MSU would induce the inflammatory response just described. Recent findings have indicated a potential beneficial role of the inflammatory response to MSU crystals.

It has long been understood that in order to induce an acquired immune response, stimulation of the immune response by an antigen must occur in the presence of an 
adjuvant or "danger signal" to amplify immune signalling and avoid tolerance (Matzinger, 1994). Bacterial and yeast cell wall components or viral RNA are known to provide the required danger signal through Toll-like receptor (TLR) signalling, which recognise the presence of foreign agents and initiate the appropriate immune defence to invading organisms (Akira et al., 2001). Dead cells are also known to provide adjuvant effects (Shi et al., 2000, Shi and Rock, 2002). Through fractionation of the components of dead cells, Rock and his colleagues identified that uric acid released from dead cells was associated with the observed adjuvant effect (Shi et al., 2003). The presence of uric acid was able to activate dendritic cells and facilitate the expansion of CD8 T-cells. This adjuvant effect was only observed when uric acid was present at concentrations high enough for crystals to form $(7 \mathrm{mg} / \mathrm{dL})$. Not only does this identify the important function of uric acid to provide a danger signal around areas of tissue damage, it also suggests that the response to MSU in a gout attack may simply occur as a result of an overactive adjuvant response that, in the absence of any secondary antigenic stimulus, resolves of its own accord.

\subsubsection{Innate cell recognition of MSU to induce an inflammatory response.}

In order for inflammation or a "danger signal" to be initiated by innate cells responding to MSU, there must be some means of recognition of MSU by innate cells. In part, some of the inflammation can be due to an indirect activation of cells, via the cleaving and activation of complement proteins at the surface of MSU crystals (Russell et al., 1982). However, in the absence of serum, MSU is still able to activate immune cells to produce pro-inflammatory molecules indicating a role for direct recognition of MSU by innate cells. Currently the receptors responsible for crystal recognition are not definitively known. TLR2 and TLR4 have been shown to play a role in MSU neutrophil recruitment in a murine air pouch model (Liu-Bryan et al., 2005). Recognition of MSU by the same pattern recognition receptors that recognise bacterial endotoxin has been strengthened by evidence showing that the endotoxin scavenging receptor and TLR2/TLR4 adaptor molecule CD14 can bind directly to MSU (Scott et al., 2006). The absence of CD14 causes a substantial reduction in both neutrophil recruitment and IL-1 $\beta$ production (Scott et al., 2006). In contrary research using a murine peritoneal model of 
acute gout, TLRs were dispensable in MSU inflammation (Chen et al., 2006). As a result of these data, the exact relevance of TLRs to MSU inflammation remains unclear.

More recently, the production of IL-1 $\beta$ by macrophages has been shown to be dependent upon the rupturing of phagosomes within cells that have phagocytosed MSU crystals (Hornung et al., 2008). Clearly, cell activation by MSU must occur through more than one pathway and the local site may heavily affect the nature of the incipient response. With the identification of the importance of MSU in both inflammation and immunity, elucidation of the recognition processes for MSU is likely to be a subject of extensive research.

\subsubsection{Production of cytokines by MSU-stimulated innate cells}

Innate cells stimulated with MSU crystals will produce a number of inflammatory molecules. Neutrophils and monocytes exposed to MSU produce large amounts of IL-8, a principal chemokine in neutrophil recruitment and priming. Mice, which do not produce IL-8, fulfil these functions via other pleiotropic mediators, such as S100 proteins as well as other IL-8R agonists such as KC and MIP proteins (Lee et al., 1995, Ryckman et al., 2003b). The production of other cytokines including TNF $\alpha$ and IL-1 $\beta$ by stimulated monocytes primes neutrophils to produce superoxide and augments their recruitment by inducing increased expression of adhesion molecules on endothelial cells (Burt and Jackson, 1997, Chapman et al., 1997). The production of MCP-1 causes the further release of monocytes from the bone marrow and their subsequent recruitment to the inflammation site (Randolph and Furie, 1995, Serbina and Pamer, 2006). Hence, there are a number of cytokines that contribute to the inflammatory response in gout. 


\subsubsection{IL-1 $\beta$ and the inflammasome}

MSU crystals stimulate the secretion of IL-1 $\beta$ from macrophages (Martinon et al., 2006). Recent research has highlighted a key role for IL-1 $\beta$ in gouty inflammation whereby the blockade of IL-1 $\beta$ abrogates inflammation (Chen et al., 2006).

The cleaving of preformed stores of proIL-1 $\beta$ into active IL- $1 \beta$ requires the assembly of a multi-protein complex called the inflammasome (Martinon et al., 2002). In macrophages, the inflammasome is comprised of the intracellular pattern recognition receptor NACHT-LRR-PYD-containing protein-3 (NALP3), the accessory protein ASC, and procaspase-1. Following activation by MSU through a process that is currently unknown, NALP3 oligomerises and recruits ASC, which in turn binds procaspase-1 leading to autocatalytic processing and activation (Mariathasan et al., 2004). Activated caspase- 1 cleaves the inactive proIL-1 $\beta$ allowing it to be secreted from the cell in its active form (Martinon et al., 2002).

Mice deficient in components of the inflammasome (ASC, caspase-1 and NALP3) or in IL-1 $\beta$ receptor (IL-1R) show a reduced neutrophil recruitment (Chen et al., 2006). Furthermore, using a combination of mouse knockouts and bone marrow chimeras, Myd88 signalling induced by IL-1R engagement on non-hemopoietic cells has been shown to be the important functional activity of IL-1 $\beta$ in neutrophil recruitment (Chen et al., 2006). Although it has not yet been established, it is likely that the nonhemopoietic cells in question were endothelial cells, given that IL-1 $\beta$ has been shown to upregulate adhesion molecules on endothelial cells, a process that is crucial for neutrophil recruitment (Chapman et al., 1997).

Another study illustrating the importance of IL-1 $\beta$ in gout tested the effectiveness of IL$1 \beta$ blockade in acute gout patients. Anakinra, a recombinant version of IL-1 receptor antagonist, was administered to ten patients who had either failed, or could not tolerate standard therapies. All patients responded rapidly to this therapy, with $9 / 10$ patients showing complete resolution of signs of arthritis three days following the initiation of 
the therapy (So et al., 2007). Collectively, these findings provide a body of evidence for the importance of IL- $1 \beta$ in gout.

\subsubsection{Resolution in gout}

A characteristic feature of a gout attack is the spontaneous resolution of inflammation. The cessation of leukocyte infiltration and the reduction in neutrophil numbers to background levels occurs over the space of seven to ten days (Poor and Mituszova, 2003). Although the cause of this spontaneous resolution in gout is not conclusively known, there are lines of evidence that mononuclear phagocytes mediate resolution in gout via Transforming Growth Factor $\beta$ (TGF $\beta)$.

The first line of evidence comes from a study showing that extraneous TGF $\beta$ inhibits MSU-induced cellular infiltration in a rat model subcutaneous air pouch (Liote et al., 1996). However, this study did not identify any endogenous sources of TGF $\beta$ in relation to the gouty disease.

The second line of evidence comes from experiments by the Haskard group showing that monocyte-like primary cells and cell lines produced pro-inflammatory cytokines (e.g. IL-6, IL-1 $\beta, \mathrm{TNF} \alpha$ ) when exposed to MSU in vitro, while mature macrophage-like primary cells and cell lines showed reduced levels of these pro-inflammatory cytokines and instead produced large amounts of TGF $\beta$ (Yagnik et al., 2004). This led to the hypothesis that the differentiation state of the monocyte-macrophage dictates the nature of the response of a mononuclear phagocyte to MSU. When mixed leukocyte populations were harvested from human blisters formed by a chemical irritant, this same pattern of switching from a "pro" to an "anti" inflammatory response was also observed when these cells were stimulated with MSU ex vivo suggesting that monocytemacrophages alter their responsiveness to MSU over the course of an inflammatory response (Yagnik et al., 2004).

The third line of evidence comes from mice that lack tissue transglutaminase, Transglutaminase 2 (TG2). Along with a host of other activities, TG2 is important in 
the uptake of apoptotic cells, and as such, macrophages from $\mathrm{TG}^{-/-}$mice are defective in the ability to take up apoptotic cells (Rose et al., 2006). There appears to be some involvement of TGF $\beta$ in this system as exogenous active TGF $\beta$ restores the phagocytic capacity of $\mathrm{TG}^{-/-}$macrophages to take up apoptotic cells. Conversely, the depletion of TGF $\beta$ inhibits apoptotic cell uptake in $\mathrm{TG}^{+/+}$macrophages. In terms of gout, $\mathrm{TG} 2^{-/-}$ mice treated with MSU by intraperitoneal injection exhibited a prolonged and more intense neutrophil infiltration implying that macrophages mediate neutrophil uptake via TG2 in a system that implicates TGF $\beta$. Unexpectedly, there were no salient differences in the level of soluble TGF $\beta$ in the surrounding milieu of $\mathrm{TG}^{-/-}$and $\mathrm{TG} 2^{+/+}$mice measured over the course of the in vivo response, implying that TGF $\beta$ may have been exerting effects at levels below detection.

The fourth line of evidence for the involvement of mononuclear phagocytes in the resolution of acute gout comes from the observation that macrophages that ingest apoptotic neutrophils (but not necrotic neutrophils) produce anti-inflammatory mediators including TGF $\beta$ as well as $\mathrm{PGE}_{2}$ and IL-10, meanwhile downregulating proinflammatory cytokines (such as TNF $\alpha$ ) (Fadok et al., 1998). In a way, this fourth line of evidence incorporates all of the phenomena discussed in the above paragraphs, as mononuclear cell differentiation is likely to occur concomitantly with the accumulation of neutrophils in vivo. The question as to which of these possibilities actually occurs in gout has yet to be demonstrated in vivo.

\subsubsection{Key innate cells in gout}

It is proposed that neutrophils, monocytes and macrophages play key roles over the course of acute gout inflammation. Monocytes and macrophages are the source of important chemokines and cytokines in acute gout, while neutrophils produce inflammatory molecules such as superoxide that augment the inflammatory response and cause physiological damage. As important cells in gout, the activities of these three cell types will be investigated in this thesis. 


\subsubsection{Neutrophils}

Neutrophils, also known as polymophonuclear leukocytes (PMN), are the most numerous white blood cells in humans comprising 50-70\% of circulating white blood cells. Under homeostatic conditions they have a very short lifespan. Following release from the bone barrow, neutrophils will undergo spontaneous apoptosis within 6-10 hours (Athens et al., 1961, Dancey et al., 1976) and are cleared from the circulation by liver and splenic macrophages (Shi et al., 2001).

Neutrophils are an important cell type in host defence and are usually the first cells recruited to a site following infection or tissue damage. Once recruited to a site, they are involved in the uptake of infectious and particulate matter, pathogens and damaged tissue, and produce hazardous molecules to kill and digest the offending organisms and dead tissue (Cassatella, 1995).

\subsubsection{Neutrophil recruitment}

The recruitment of leukocytes such as neutrophils from the blood into a space such as the synovium requires a number of events that broadly fit into four stages: leukocyte rolling; leukocyte activation; leukocyte arrest; and transendothelial migration.

The first stage, leukocyte rolling, is dependent on adhesion molecules called selectins. Even under non-inflammatory conditions, free flowing neutrophils can undergo contact with endothelial cells through the interaction of selectin adhesion molecules P-selectin, E-selectin and L-selectin on leukocytes (Kansas, 1996), with P-selectin glycoprotein ligand-1 (PSGL-1) and other adhesion molecules expressed on endothelial cells (McEver and Cummings, 1997). In particular, the interaction of L-selectin with PSGL-1 allows the capture and tethering of neutrophils to the endothelial wall in a manner that is strengthened under shear stress caused by blood flow (Marshall et al., 2003). The subsequent rolling of leukocytes along the endothelium is achieved through the formation of new selectin-PSGL-1 interactions concomitant with the breaking of old ones (Yago et al., 2007 McEver, \& Zhu, 2007). 
The activation of leukocytes at the next stage is connected to the activation of endothelial cells. Under inflammatory situations, endothelial cells are stimulated by cytokines to express adhesion molecules and present chemoattractants on their luminal surface (Campbell et al., 1998, Campbell et al., 1996). These chemoattractants can be produced directly by the endothelial cells or generated at the site of inflammation - for instance, by activated mast cells - and transported to endothelial cells as circulating microparticles (Huo et al., 2003, von Hundelshausen et al., 2001). Chemoattractants transferred to the luminal endothelium may be found bound to glycosaminoglycans, which allow chemoattractants to be presented to rolling leukocytes (Johnson et al., 2005 ).

Exposure of leukocytes and the endothelium to chemokines activates both cell types and leads to the rapid arrest of leukocyte rolling. Leukocyte arrect is dependent on integrins, including $\beta_{1}$-integrin VLA4, and the $\beta_{2}$-integrin LFA1 (Constantin et al., 2000, Laudanna et al., 2002, Shamri et al., 2005). Integrins are typically present on leukocytes in an inactive conformation, however, chemoattractant stimulation of leukocytes induces cellular signals via specific G-protein-coupled receptors that lead to a rapid conformational change of integins. The altered integrin structures exhibit a highly increased affinity for their cognate molecules on endothelial cells (Constantin et al., 2000, Laudanna et al., 2002, Shamri et al., 2005). Typically, these cognate molecules are immunoglobulin super family members such as ICAM1 and VCAM1 (Campbell et al., 1998, Campbell et al., 1996). Interactions between integrins with ICAM1 and VCAM1 are strong, and lead to full arrest of leukocytes.

The final stage involves the movement of leukocytes through endothelial cells and the basement membrane. Neutrophils begin to crawl through endothelial cells junctions in a manner that is dependent on MAC1 and ICAM1 (Phillipson et al., 2006, Schenkel et al., 2004). Signalling through ICAM1 causes the release of intracellular $\mathrm{Ca}^{2+}$, the activation of p38 mitogen activated protein kinase (MAPK) and RAS homologue (RHO) GTPase which together leads to endothelial contraction facilitating the opening of interendothelial junctions (Greenwood et al., 2003, Huang et al., 1993, Millan and Ridley, 
2005, Muller, 2003). This is further aided by the release of inter-endothelial cell adhesion, through the diminishment of contact of vascular endothelial cadherin (VEcadherin) on neighbouring endothelial cells (Shaw et al., 2001). Adhesion molecules within the junction promote leukocyte-endothelial cell interactions. Transmigration through the junction occurs through a number of adhesion molecules, including homophilic interactions of platelet/endothelial cell adhesion molecule 1 (PECAM1) (Petri and Bixel, 2006), and also CD99, as well as interactions of junction adhesion molecules (JAMs) with LFA1, VLA4, MAC1 (Lou et al., 2007, Mamdouh et al., 2003, Muller, 2003, Nourshargh et al., 2006 , Schenkel et al., 2002, Vestweber, 2002). Finally, movement through the basement membrane occurs at gaps between pericytes (cells wrapped around post-capillary venules) and at areas of low protein deposition within the extracellular matrix (Sixt et al., 2001, Wang et al., 2006). Movement of neutrophils through the basement membrane and between pericytes appears to involve $\alpha 6 \beta 1$-integrin (Dangerfield et al., 2002) as well as proteases such as matrix metalloproteinases (Adair-Kirk et al., 2003) and neutrophil elastase (Wang et al., 2005) expressed on the cell surface.

\subsubsection{Recruitment of neutrophils in gout}

A neutrophil rich synovial infiltrate is a feature of a gout attack where particular cytokines and chemokines have been shown to play a pivotal role in neutrophil recruitment. Animal models of gout have shown an absolute requirement for the IL-8 receptor (CXCR-2) (Terkeltaub et al., 1998) and of IL-1 $\beta$ (Chen et al., 2006, Martinon et al., 2006) in the recruitment of neutrophils, while other molecules such as TNFa, GM-CSF (Hachicha et al., 1995) S100A8 and S100A9 (Ryckman et al., 2003a) and complement proteins (Tramontini et al., 2004, Russell et al., 1982) are thought to augment recruitment. One of the major roles of these soluble mediators is the upregulation of adhesion. Indeed, TNF $\alpha$ and IL-1 $\beta$ generated by MSU-activated monocytes has been shown to upregulate the adhesion molecules E-selectin, ICAM-1 and VCAM-1 on human endothelial cells allowing the tethering of neutrophils to endothelial cells in vitro (Chapman et al., 1997). 
Several cell types produce the chemokines and cytokines required for neutrophil recruitment in gout. MSU-induced IL-8 is likely to come from a number of sources including monocytes, macrophages and neutrophils (Terkeltaub et al., 1991, Hachicha et al., 1995). Macrophages are a potential source of IL-1 $\beta$ in gout (Martinon et al., 2006) while mast cells also contribute to neutrophil recruitment in MSU inflammation through histamine and PAF release (Getting et al., 1997). These studies indicate that the recruitment of neutrophils is dependent on a number of cell types that collectively contribute to the overall neutrophil response in acute gout.

\subsubsection{Activation and survival of neutrophils in inflammation}

Exposure of neutrophils to cytokines and growth factors (IL-1 $\beta$, TNF $\alpha$, IFN $\gamma, \mathrm{G}-\mathrm{CSF}$, GM-CSF) at the site of inflammation has been shown to prolong the survival of neutrophils from several hours to several days in vitro (Akgul et al., 2001). The implication of this is that neutrophils that would usually be short-lived will persist in the inflamed tissue for an extended period of time allowing for the accumulation of sizeable numbers of neutrophils at an inflammatory site and therefore a prolonged proinflammatory activity.

The neutrophil receptors that recognise MSU are currently unknown. In other inflammatory conditions, the recognition of foreign particles, bacteria, or other inflammatory agents occurs via pattern recognition receptors such as TLRs and NLRs (Ozinsky et al., 2000, Martinon and Tschopp, 2005). Foreign agents may also be detected and phagocytosed by first becoming opsonised with antibodies and complement factors that allows the particle to be taken up into the neutrophil through interactions with $\mathrm{F}_{\mathrm{c}}$ and complement receptors respectively (Tjelle et al., 2000).

The result of phagocytic uptake is the containment of the particle within specialised structures called phagosomes (Tjelle et al., 2000). Phagosomes are formed during the invagination of the membrane and the remodelling of the neutrophil actin cytoskeleton around the foreign particle during internalisation. Phagosomes are only slightly larger than the ingested particle and become the focus site for intracellular inflammatory attack 
and pathogen neutralisation (Rozenberg-Arska et al., 1985). Cytoplasmic granules fuse with the phagosome to release proteolytic enzymes and bactericidal proteins into the phagosome in a process called degranulation (Hirsch and Cohn, 1960). The respiratory burst enzyme, NADPH oxidase, also assembles at the phagosome membrane to produce reactive oxygen species (Hampton et al., 1998). In addition to superoxide production within the phagosome, the activation of NADPH oxidase also leads to the production of extracellular superoxide from the cell surface that can also inflict biological damage upon microbes as well as surrounding tissue (Tan and Berridge, 2000).

The recognition and phagocytosis of inflammatory particles initiates the production and release of additional cellular pro-inflammatory cytokines, most commonly IL-8 and S100A8/A9, which recruit further neutrophils to amplify the inflammatory response (Harada et al., 1994, Ryckman et al., 2003b). The level of cytokines such as IL-8 is commonly used as a measure of neutrophil activation (Harada et al., 1994).

\subsubsection{Production of superoxide}

A fundamental function of neutrophils in inflammation is the production of superoxide in a process called the "respiratory burst." Superoxide $\left(\mathrm{O}_{2}{ }^{--}\right)$is a major component of host defence and plays a pivotal role in the generation of all the other reactive oxygen species (Fig. 1.6) (Segal, 2005). Superoxide itself is only a mild oxidant. However, spontaneous and enzyme mediated dismutation of superoxide to hydrogen peroxide $\left(\mathrm{H}_{2} \mathrm{O}_{2}\right)$ facilitates the production of much more powerful oxidants. For instance, $\mathrm{H}_{2} \mathrm{O}_{2}$ forms the highly reactive hydroxyl radial $\left(\mathrm{OH}^{*}\right)$ in the presence of transition metals such as iron; and $\mathrm{H}_{2} \mathrm{O}_{2}$ also forms hypohalous acids such as hypochlorous acid ( $\mathrm{HOCl}$ ) after reacting with halide ions in the presence of myeloperoxidase (Fig. 1.7). In addition, superoxide can react with nitric oxide (NO) to produce highly reactive peroxynitrite $\left(\mathrm{ONOO}^{\circ}\right)$ (Fig. 1.7). The primary role of these ROS is as bactericidal and

fungicidal molecules (Segal, 2005). The importance of these compounds is illustrated in Chronic Granulomatous Disease (CGD), a rare genetic disorder whereby neutrophils are 


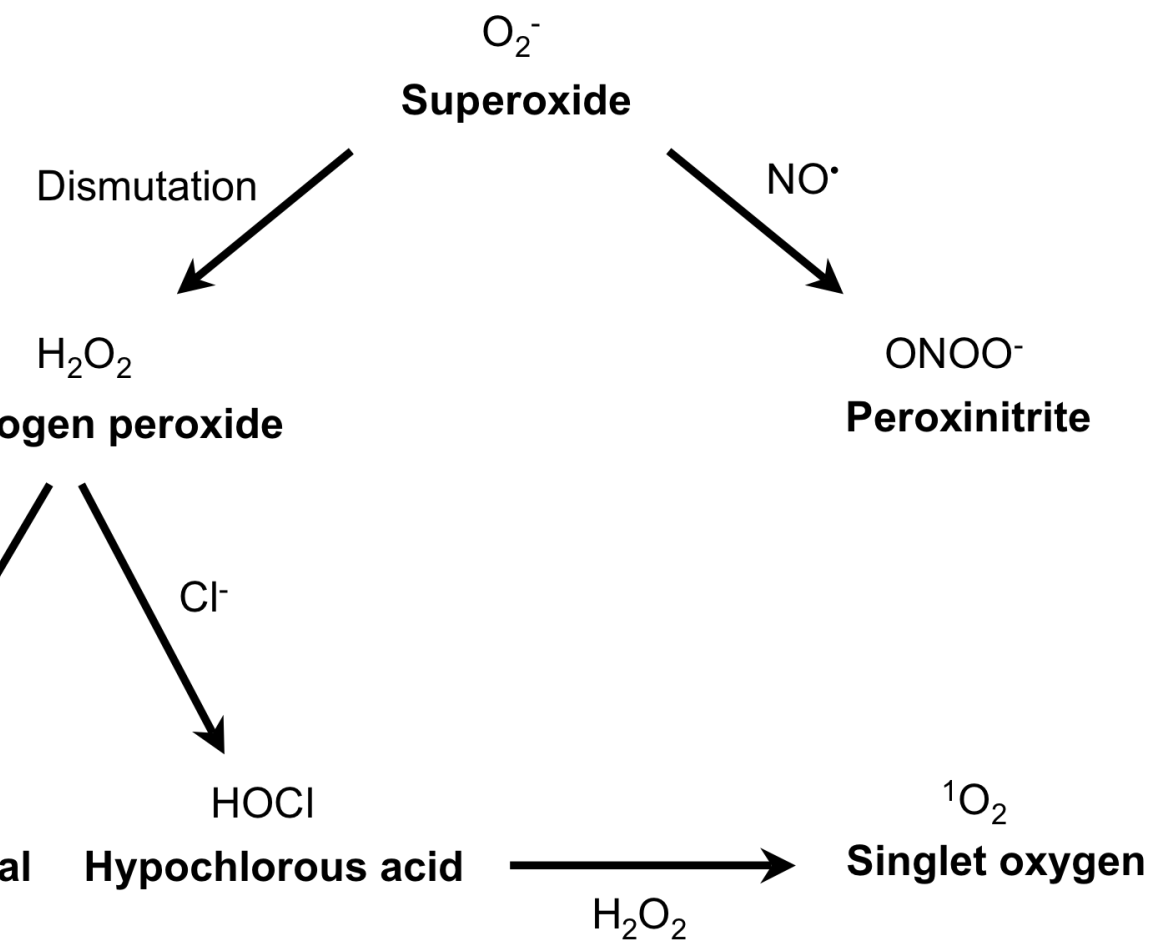

Figure 1.6. The formation of reactive oxygen species is dependent on superoxide. This flow chart summarises how superoxide is used to generate the other reactive oxygen species. 
unable to produce a respiratory burst and the subsequent ROS. As a result, CGD individuals frequently suffer from life threatening bacterial and fungal infections (Segal et al., 2000).

\subsubsection{Collateral damage through excessive ROS production}

The down side of ROS production and indeed, of other neutrophil inflammatory activities, is the collateral damage caused to the host during defensive ROS generation. Classically, NADPH oxidase activation occurs following contact and phagocytosis of foreign particles or bacteria and, as this process occurs within the phagosome, a significant amount of superoxide production is intracellular (Hampton et al., 1998). However, high levels of extracellular superoxide can also be measured following the activation of neutrophils. In addition to this, $\mathrm{H}_{2} \mathrm{O}_{2}$ formed by superoxide dismutation is cell permeable and can pass into the extracellular space. Errant ROS are indeed problematic, leading to oxidation of host enzymes, proteins and lipids (a process that itself induces a chain reaction of further oxidation), and ultimately tissue damage, as observed in the joint during inflammatory arthritis (Kroger et al., 1996, Miesel et al., 1996). This problem is particularly relevant in chronic inflammation and in situations in which the generation of superoxide is physiologically irrelevant, such as in gout, where MSU triggers a sterile inflammatory response.

\subsubsection{Neutrophil investigation}

Neutrophils are recruited in high numbers in gout and are known to produce large amounts of superoxide in addition to pro-inflammatory cytokines following exposure to MSU while the intensity of neutrophil infiltration is considered a correlate of the severity of gout response in animal models. Since neutrophils are able to induce damage to host tissues, the recruitment of neutrophils and their activation has been a subject of interest in gout research. 
Generation of superoxide:

$$
2 \mathrm{O}_{2}+\mathrm{NADPH}
$$

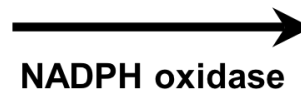

$2 \mathrm{O}_{2}^{-}+\mathrm{NADP}^{+}+\mathrm{H}^{+}$

Production of reactive molecules from superoxide:

$$
\begin{gathered}
2 \mathrm{O}_{2}^{-}+2 \mathrm{H}^{+} \underset{\text { Superoxide dismutase }}{\longrightarrow} \mathrm{O}_{2}+\mathrm{H}_{2} \mathrm{O}_{2} \\
\mathrm{O}_{2}^{-}+\mathrm{NO}^{-} \longrightarrow \mathrm{ONOO}^{-}
\end{gathered}
$$

Production of reactive molecules from hydrogen peroxide:

$$
\begin{array}{lll}
\mathrm{H}_{2} \mathrm{O}_{2}+\mathrm{Cl}^{-} & \text {Myeloperoxidase } & \mathrm{OCl}^{-}+\mathrm{H}_{2} \mathrm{O} \\
\mathrm{H}_{2} \mathrm{O}_{2}+\mathrm{Fe}^{2+} & \longrightarrow \mathrm{OH}^{-}+\mathrm{OH}^{-}+\mathrm{Fe}^{3+} \\
\mathrm{H}_{2} \mathrm{O}_{2}+\mathrm{OCl}^{-} & \longrightarrow{ }^{1} \mathrm{O}_{2}+\mathrm{H}_{2} \mathrm{O}+\mathrm{Cl}^{-}
\end{array}
$$

Figure 1.7. Equations outlining the reactions in the formation of ROS and the enzymes involved. 
A common research approach has been to use purified human blood neutrophils, yet neutrophils are never an isolated player during an inflammatory gout response. Therefore, a major area for investigation is how this cell fits into the network of cellular responses during a gout attack, working in concert and cooperation with other inflammatory cells, such as monocytes and macrophages.

\subsubsection{The mononuclear phagocyte system}

Monocytes and macrophages are part of a highly diverse group of cells that make up the mononuclear phagocyte system (MPS). The MPS is a set of related cells that includes blood monocytes, tissue macrophages, kupffer cells, langerhans cells, dendritic cells, osteoclasts, and microglia (Hume et al., 2002). These cells play a key role in inflammation and host defence, mediating a wide number of processes from phagocytosis to antigen presentation, production of anti- and pro-inflammatory mediators, immunosuppression and tissue repair. In gouty arthritis, monocytes and macrophages appear to play a key role in both pro- and anti-inflammatory responses to MSU.

\subsubsection{Monocytes}

Monocytes are phagocytic mononuclear cells that comprise approximately 5\% of white blood cells in circulation. Although they can respond to inflammatory stimuli such as LPS and produce inflammatory mediators (e.g. IL-8, TNF $\alpha$, IL-6) they are considered to be an immature precursor cell (van Furth and Cohn, 1968). Once monocytes are recruited from the circulation into tissues they have the capacity to differentiate into a number of cell types depending on factors including tissue type (joint, skin, bone, brain) and the chemical mediators present in the local environment. The developmental sensitivity of monocytes to chemical mediators is clearly displayed in vitro where monocytes cultured in M-CSF will predominantly differentiate into macrophages (Warren and Vogel, 1985), monocytes cultured in GM-CSF and IL-4 will become dendritic cells (Zhou and Tedder, 1996), while monocytes cultured with RANKL and M-CSF become osteoclasts (Udagawa et al., 1990). 
Monocytes consist of two populations that are easily distinguished from each other using a combination of markers such as Gr-1 and 7/4 in mice, and CD14 and CD16 in humans (Geissmann et al., 2003). Additional markers $\mathrm{CX}_{3} \mathrm{CR} 1, \mathrm{CDC} 62 \mathrm{~L}$ and CCR2 can also be used (Table 1.1). When monocytes first egress the bone marrow they are $\mathrm{Gr}^{+} 1^{+}$(mouse) or $\mathrm{CD}_{1} 4^{+}$(human). Large numbers of these monocytes are recruited to sites of inflammation and so have been termed "inflammatory" monocytes (Geissmann et al., 2003). Although inflammatory monocytes can differentiate into both macrophages and dendritic cells in vitro, they appear to be predisposed to becoming dendritic cells following an in vivo insult, such as Listeria monocytogenes (Auffray et al., 2007). This is supported by experiments showing that these monocytes are also responsible for replenishing langerhans cells following an inflammatory insult to the skin (Ginhoux et al., 2006).

The second population of monocytes are $\mathrm{Gr}-1^{-}$in mouse and $\mathrm{CD} 14^{\text {lo }}$ in humans. Studies depleting circulating monocytes and observing monocyte reconstitution suggest that Gr$1^{-}$monocytes are derived from Gr- $1^{+}$monocytes (Sunderkotter et al., 2004). Adoptive transfer studies have shown that Gr-1 monocytes invade most tissues under homeostatic conditions and are thus thought to replenish resident macrophage and dendritic cell populations under steady state conditions (Geissmann et al., 2003). Due to this they are termed "resident monocytes." Resident monocytes have also been shown to patrol the vascular wall of blood vessels and respond to inflammatory stimuli such as $L$. monocytogenes in vivo (Auffray et al., 2007). Resident monocytes can differentiate into dendritic cells in vitro with exposure to GM-CSF and IL-4, but gene expression profiles show that these monocytes are predisposed to becoming macrophages following an inflammatory challenge in vivo (Auffray et al., 2007). 


\begin{tabular}{|c|c|c|}
\cline { 2 - 3 } \multicolumn{1}{c|}{} & \multicolumn{2}{c|}{ Human monocytes } \\
\cline { 2 - 3 } \multicolumn{1}{c|}{} & Resident & Inflammatory \\
\hline CD14 & $\mathrm{lo}$ & $\mathrm{hi}$ \\
CD16 (FCgRII) & + & - \\
CX ${ }_{3}$ CR1 & $\mathrm{hi}$ & $\mathrm{lo}$ \\
CCR2 & - & + \\
CD62L & - & + \\
\hline
\end{tabular}

\begin{tabular}{|c|c|c|}
\cline { 2 - 3 } \multicolumn{1}{c|}{} & \multicolumn{2}{c|}{ Mouse monocytes } \\
\cline { 2 - 3 } \multicolumn{1}{c|}{} & Resident & Inflammatory \\
\hline Ly-6C $(\mathrm{Gr}-1)$ & - & + \\
$7 / 4$ & - & + \\
$\mathrm{CX}_{3} \mathrm{CR} 1$ & $\mathrm{hi}$ & $\mathrm{lo}$ \\
$\mathrm{CCR} 2$ & - & + \\
$\mathrm{CD} 62 \mathrm{~L}$ & - & + \\
\hline
\end{tabular}

Table 1.1. Expression levels of selected markers on resident and inflammatory monocytes expression markers. Expression of human and mouse monocytes markers are shown. Information taken from Gordon 2005. 
Although monocytes can respond to inflammatory stimuli, their main contribution is likely associated with the cell type into which they differentiate following recruitment. For instance, dendritic cells will be efficient at presenting antigen, influencing Tcellresponses and engaging the adaptive immune response (Banchereau and Steinman, 1998); whereas macrophages will efficiently phagocytose pathogens and initiate a range of responses from inflammatory mediator production, to bactericidal activities, to tissue repair (Hume et al., 2002); while osteoclasts will adhere to and resorb bone (Udagawa et al., 1990). The factors that govern the differentiation fate of monocytes in vivo are complicated, yet the influence of those factors on monocytes is intimately involved in the nature of an inflammatory response. Therefore, an important aspect to understanding an inflammatory disease is to understand monocyte fate and function within the context of that disease.

\subsubsection{Monocytes in gout}

Understanding the role of monocytes in gout has come almost exclusively from in vitro experiments with murine cell lines and human blood monocytes. In one study, a number of murine monocyte-macrophage cell lines were put in an order of differentiation status based on the expression of the F4/80 antigen, then stimulated with MSU (Yagnik et al., 2000). Based on this arrangement, cell lines that were more monocyte-like produced large amounts of TNF $\alpha$ when exposed to MSU, while the cell lines that were more macrophage-like did not. In a follow up study, freshly isolated $\mathrm{CD} 14^{+}$human blood monocytes produced TNF $\alpha$ and IL- $\beta$ when stimulated with a single dose of MSU. However, $\mathrm{CD} 14^{+}$monocytes were allowed to differentiate into macrophages by simple cell adhesion and stimulated with MSU crystals subsequently lost the ability to produce TNF $\alpha$ and IL- $\beta$ and began to produce TGF $\beta$, indicating a switch from a proinflammatory to an anti-inflammatory phenotype (Landis et al., 2002, Yagnik et al., 2004). This led to the idea that monocytes interacting with MSU drive the inflammatory phase in gout, while macrophages are involved in resolution (Fig. 1.8). 


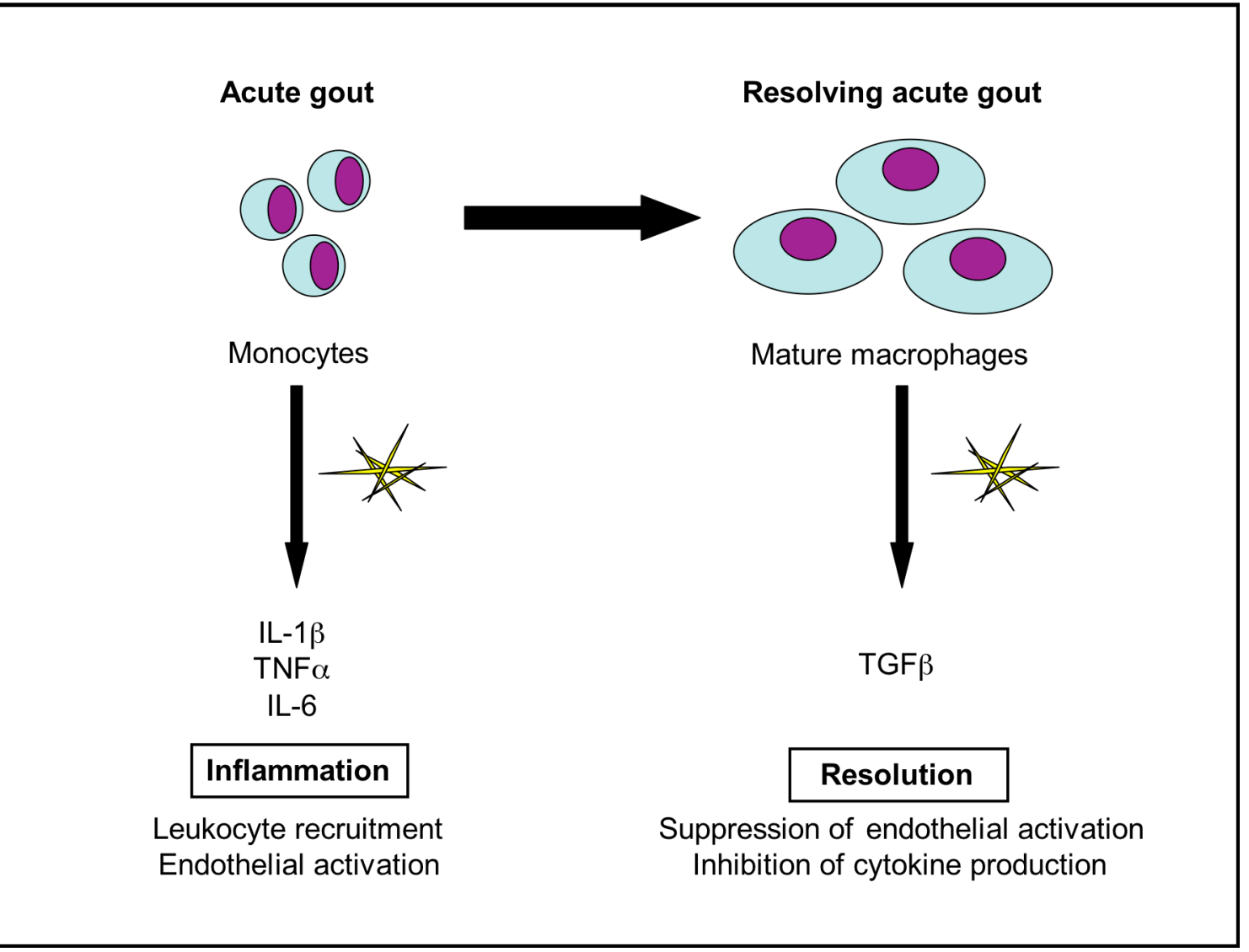

Figure 1.8 A proposed model for the involvement of monocytes and macrophages in acute gout. The Haskard model of acute gouty inflammation proposes monocytes to be key inflammatory cells in the initiation of acute gouty inflammation, producing proinflammatory cytokines in response to MSU crystals; and mature macrophages to be important in the resolution of gout through the production of TGF $\beta$ following exposure to MSU. Figure adjusted from Dalbeth and Haskard, 2005. 
In the only in vivo based model used to demonstrate the response of monocytes to MSU, skin blisters were induced on human skin using the irritant cantharidin. During the course of the inflammatory response unpurified leukocyte infiltrates were removed from the blisters and exposed to MSU (Yagnik et al., 2004). Restimulation of the heterogeneous cell populations with MSU exhibited the generation of TNF $\alpha$ from $16 \mathrm{~h}$ blisters, whereas cells from $48 \mathrm{~h}$ blisters produced no TNF $\alpha$ and instead produced antiinflammatory TGF $\beta$. Although these cells exhibited a cytokine 'switching' following stimulation with MSU in vitro, no phenotyping of the cell types was undertaken.

The results from the above in vitro experiments show consistency but have clear limitations. First, the primary criterion used to classify the murine cell lines as monocyte- or macrophages-like was a combination of F4/80 and BM8 expression, yet it is now known that the BM8 antibody also recognises the F4/80 antigen (Schaller et al., 2002). A close examination of the F4/80 expression levels within the study also reveals that there was very little difference between most of the cells lines, making F4/80 a poor correlate of differentiation between these cells (Yagnik et al., 2000). In the absence of appropriate differentiation or activation markers it is not possible to ascribe functions to stages of differentiation. This lack of appropriate phenotypic markers also applies to $\mathrm{CD}^{+} 4^{+}$monocytes and cantharidin induced leukocytes, for which there was no characterisation of phenotype.

A second limitation is that the in vitro studies cannot account for the phenotypic changes that occur during recruitment. Interaction of monocytes with endothelium leads to an increase in expression of several genes in monocytes, including MMP-1, MCP-1, TG2 caveolin-1 and CD4 (Thomas-Ecker et al., 2007). Infiltrating monocytes are exposed to a complex mixture of cytokines, serum exudate, cells and tissues which provide a very different condition for differentiation than those provided in vitro, where monocytes are routinely differentiated only using M-CSF, GM-CSF or by adherence to plastic plates (Fleetwood et al., 2007). 
Together these factors indicate that cell lines and in vitro differentiated monocytes are potentially very different to monocytes recruited in vivo. This hypothesis requires examination in a relevant in vivo model.

\subsubsection{Macrophages}

The term macrophage refers to a highly diverse cell type that is found in most tissues throughout the body that share a common stem cell progenitor. They include kupffer cells of the liver, alveolar macrophages of the lung, serosal macrophages of the peritoneum, type A cells in the joint synovium, histiocytes of connective tissue, and microglia of the central nervous system (Gordon and Taylor, 2005). Although they can differ greatly in their phenotype and responsiveness depending on their environment, they share common cardinal features including phagocytosis, cytokine and chemokine production (IL-1 $\beta$, TNF $\alpha$, IL-6, IL-12, IL-10, IFN $\gamma$, etc) (Leenen and Campbell, 1993). They are often identified by the expression of the M-CSF receptor (Sasmono et al., 2003, Sweet and Hume, 2003). In mice, F4/80 is a marker for macrophage identification (Hume et al., 1983).

Macrophages are laid down in tissues during oocytogenesis and are either maintained by local progenitor cells or replenished by circulating monocytes during steady state conditions (Lichanska and Hume, 2000). During inflammatory conditions it is believed that they are replenished solely by monocytes (Geissmann et al., 2003).

The most characteristic feature of macrophages is a high level of plasticity in terms of the range of responses and phenotypes they are able to employ. Macrophages are very sensitive to environmental signals and will adjust their responses according to stimuli that are present (Stout and Suttles, 2004). In this way, the specific response of macrophages to a particular stimulus can depend on a complex interplay between the stimulus, the extracellular signals in the surrounding milieu, the length of time that the macrophage has been exposed to those signals, and the sequential changes in the microenvironment, including those initiated by the macrophage itself (Leenen and Campbell, 1993). The complexity of possible responses from macrophages necessitates 
the investigation of macrophage responses in an environment that faithfully reflects the inflammatory condition that is being modelled.

\subsubsection{Macrophages in gout}

Based on in vitro studies outlined in Section 1.10.10.1, macrophages are thought to be involved in the resolution of a gout attack, through the production of the antiinflammatory molecule TGF $\beta$ and the absence of pro-inflammatory cytokines following stimulation with MSU. However, macrophages have also been reported to produce IL$1 \beta$, TNF $\alpha$, MCP-1, IL-18, iNOS and upregulate the expression of TREM-1 in response to MSU (Jaramillo et al., 2004a, Jaramillo et al., 2004b, Chen et al., 2006, Martinon et al., 2006, Murakami et al., 2006). These responses suggest the potential for macrophages to play a pro-inflammatory role in vivo. These contrasting reports highlight that macrophage function in gout is still not clearly understood, and that further investigation into this area is needed.

\subsubsection{Using research models to investigate gout}

In order to study acute gouty inflammation, it has been essential to develop research models. The first substantiated animal model for gout was developed by Faires and McCarty, in which they injected synthetic MSU into dog knees (Faires and McCarty, 1962). Using this model, they were able to reproduce the same symptoms that they had observed when they had self administrated MSU, such as pain and joint swelling and cellular infiltration. Following up on this experiment McCarty used this model to show that the intensity of pressure within the inflamed joint correlated to neutrophil infiltration and was dependent on the dose of MSU administered (McCarty et al., 1966, Phelps and McCarty, 1966). This not only confirmed MSU as the direct etiological agent in gout but also corroborated the use of animal models to study gout. Since then, hamster and rabbit joints have also been used as models of gouty inflammation.

In the last 20 years, mouse and rat models have also been developed to study gout. The size of the murine joint precludes an effective study of gout by intrasynovial injection of MSU, and so murine models have been established using alternative tissue sites to 
mimic the joint synovial space. In these models, either the peritoneum or the subepidermis is used as an MSU injection site to follow inflammatory responses to MSU.

\subsubsection{Murine peritoneal model of gout}

The peritoneal model was first used in gout research by Getting and colleagues (Getting et al., 1997). The peritoneum contains cell types that are similar in nature to cells in the joint, including macrophages, epithelial cells, mast cells, fibroblasts and lymphocytes. In this model, MSU crystals are injected into the peritoneum which induces a strong leukocyte infiltration, including neutrophils, as well as the production of cytokines and chemokines known to be associated with gout. A limitation of this model is the absence of certain cell types that are specific to bone niches including osteoclasts and chondrocytes, and tissue such as cartilage and bone. The relatively large amount of space within the peritoneum also prevents the observation of mechanically induced algesia due to oedema, a phenomenon that accompanies a gout attack within the enclosed space of the joint. Despite these disadvantages, outcomes of this model have been carried through to clinical studies in gout confirming the usefulness and relevance of this model.

\subsubsection{Murine air pouch model}

In the murine air pouch model a sac is formed on the back of rats (or mice) by a subcutaneous injection of sterile air several days before MSU challenge. This faux space is formed to mimic the joint synovium. Injection of MSU into this space induces the recruitment of leukocytes. A description of MSU-induced recruitment in this model has been reported by Schiltz and colleagues (Schiltz et al., 2002).

This model has the same limitation as the peritoneal model: an absence of a few cell types that would be found in the bone niche. However, another limitation of this model is that the inflammation caused by the formation of the air pouch may set up a background inflammatory environment, either in cell number or cell phenotype, which may affect subsequent inflammatory challenges. In this way, it is difficult to compare 
the quality of MSU induced inflammation in a previously uninflamed environment, as would be expected in an acute attack of gout.

In this research, the MSU-peritonitis model has been chosen as the inflammatory model for gout. This is due to my interest in the onset of acute gout against an unmanipulated naïve background of known phenotype. It induces a cellular infiltration that mimics clinical gout, is widely used in the literature and has shown relevancy in clinical followthrough experiments. For these reasons I feel confident that the findings from the use of this model will be relevant to acute gouty disease.

\subsection{Aims of this study}

The research summarised in this chapter implicates neutrophils, monocytes and macrophages as key players in acute gout. It is my intent that this thesis will deepen and clarify the roles of these cells in acute gout and address aspects of cellular inflammation in gout that require further investigation.

The aims of this study were:

Aim 1 To determine whether inflammatory factors - other than MSU - that are present in the inflammatory environment during an acute gout attack contribute to the activation of neutrophils.

Aim 2 To assess whether neutrophil activation in MSU-inflammation can be inhibited by the novel anti-inflammatory compounds, sesquiterpene dialdehydes.

Aim 3 To determine the role of monocytes and macrophages in the initiation of MSU-induced inflammation in vivo.

Aim 4 To profile the differentiation pathway of MSU-elicited monocytes during the course of acute MSU-induced inflammation in vivo. 
Chapter 2

Materials and Methods 


\section{Chapter 2}

\section{Materials and Methods}

\subsection{Reagents}

\section{Buffer/Media}

Dulbecco's Phosphate-Buffered Saline (D-PBS)

Hanks’ Balanced Salt Solution (HBSS)

Medium 199 (M199)

Phosphate Buffered Saline (PBS)

RPMI-1640

\section{Source}

Invitrogen, $\mathrm{NZ}$

Invitrogen, NZ,

Sigma-Aldrich, NZ

Invitrogen, NZ

Invitrogen, NZ

Invitrogen, NZ

\section{Chemical/Miscellaneous materials Source}

\section{$0.25 \%$ trypsin/EDTA}

$10 \%$ neutral buffered formalin

Acetone

Alexa488 Streptavidin

Alexa555 Streptavidin

Bovine fibroblast growth factor (bFGF)

Bovine serum albumin (Fraction V, IgG free)

Calcium chloride

Clodronate

Dimethyl sulfoxide (DMSO)

Diphenyleneiodonium chloride (DPI)

DNaseI (Grade II)

Epidermal growth factor

Ethanol
Invitrogen, NZ

Sigma-Aldrich, NZ

BDH chemicals NZ Ltd.

Invitrogen, NZ

Invitrogen, NZ

Invitrogen, NZ

Invitrogen, NZ

BDH chemicals NZ Ltd.

Sigma-Aldrich, NZ

Sigma-Aldrich, NZ

Biolab Scientific, Australia

Roche, NZ

Roche, NZ

BDH chemicals NZ Ltd. 
Fetal bovine serum

FITC Annexin-V

FITC Streptavidin

FluoSpheres fluorescent microspheres

(0.5um; 505/515nm; 2\% solids)

Gelatin

Glutamax

GolgiStop

Heparin

HEPES

Hoechst 33342 (bisbenzamide)

Horseradish peroxidase

Hydrochloric acid

L-a-Phosphatidylcholine

Lipopolysaccharide, E.coli 0111:B4 (LPS)

Luminol

Macrophage colony stimulating factor (M-CSF)

Methanol

$o$-dianisidine

Penicillin-streptomycin

Phorbol 12-myristate 13-acetate (PMA)

Polymorphprep

Propidium iodide

Puregene RBC lysis buffer

R-PE Streptavidin

Saponin

Sodium azide

Sodium chloride

Sodium chloride 5M (Cell culture and endotoxin tested)

Sodium hydroxide
Sigma-Aldrich, NZ,

Invitrogen, NZ

BD Bioscience, NZ

eBioscience, San Diego, USA

Invitrogen, NZ

Merck, Australia

Invitrogen, NZ

BD Bioscience, NZ

Wellington Hospital, NZ

Sigma-Aldrich, NZ

Sigma-Aldrich, NZ

Sigma-Aldrich, NZ

BDH chemicals NZ Ltd.

Sigma-Aldrich, NZ

Biolab Scientific, Australia

Sigma-Aldrich, NZ

Peprotech, New Jersey, USA

BDH chemicals NZ Ltd.

Sigma-Aldrich, NZ

Invitrogen, NZ

Sigma-Aldrich, NZ

Medica Pacifica Ltd.

BD Bioscience, NZ

ProGENZ, NZ

eBioscience, San Diego, USA

Sigma-Aldrich, NZ

Sigma-Aldrich, NZ

BDH chemicals NZ Ltd.

Sigma-Aldrich, NZ

BDH chemicals NZ Ltd. 
Thioglycollate Medium

Uric acid

WST-1

Zinc fixative
Difco, Detroit, MI, USA

Sigma-Aldrich, NZ

Dojindo, Kumamoto, Japan

BD Bioscience, NZ

\subsubsection{Sesquiterpene dialdehydes}

The dialdehyde compounds used in this study and shown in Figure 4.1 were kindly provided by Nigel Perry of the Plant and Extracts Research Unit (PERU) at Plant and Food Research in Otago, New Zealand.

\subsection{Antibodies}

All antibodies for use in flow cytometry or immunofluorescent histology were titrated before use to determine the ideal concentration for use. Antibodies of the same isotype as the treatment antibody, conjugated to the same fluorophore as the treatment antibodies, yet with specificity to an irrelevant, non-present antigen were used as control in stainings.

\section{BD Bioscience (NZ)}

APC Mouse anti human CD15

APC Hamster anti mouse CD11c

APC Rat anti mouse Gr-1

Mouse $\operatorname{IgG} 2_{\mathrm{b}}, \mathrm{k}$ Isotype control

PerCP-Cy5.5 Rat anti mouse CD11b

R-PE Armenian hamster $\operatorname{IgG}_{1}, \mathrm{k}$ Isotoype control

R-PE Hamster anti mouse MCP-1

R-PE Mouse anti human IL-8

R-PE Rat anti mouse IL-6

R-PE Rat $\operatorname{IgG}_{1}$ Isotype control 


\section{eBioscience (San Diego, USA)}

Anti mouse IL-6

Armenian hamster anti mouse/rat IL-1 $\beta$

Biotin Mouse anti-rat IgG

Biotin Rabbit anti mouse IL-1 $\beta$

Biotin Rat anti mouse IL-6

Biotin Rat $\operatorname{IgG}_{2 \mathrm{a}}$ Isotype control

FITC Goat anti armenian hamster IgG

Rat anti mouse TNF $\alpha$

Rat $\mathrm{IgG}_{1}$ Isotype control

R-PE Rat anti mouse F4/80

R-PE Rat anti mouse TNF $\alpha$

\section{Serotec (Oxford,UK)}

Alexa488 Rat anti mouse F4/80

FITC Rat anti mouse CD68

FITC Rat anti mouse neutrophils (7/4)

R-PE Rat anti mouse CD206

\section{Other}

Anti-mouse FcyRII antibody (24g.2) was kindly provided by Thomas Backstrom from the Malaghan Institute of Medical Research.

\subsection{Kits}

Biotin blocking kit

Bioplex bead array kits

Cytokine bead array (CBA) kits

\section{Source}

Invitrogen, NZ

Biorad, NZ

BD Bioscience, NZ 
Diff-Quik kit

IL-1 $\beta$ ELISA kit

Limulus amebocyte lysate kit

Lincoplex bead array kits

PKH26 red fluorescent phagocytic cell linker kit

TNF $\alpha$ and IL-6 OptiEIA ELISA kits
Dade Behring, Newark, USA

R\&D Systems, Minneapolis, MN, USA

Associates of Cape Cod, Inc, Falmouth, MA, USA

Abacus ALS, NZ

Sigma-Aldrich, NZ

BD Bioscience, NZ

\subsection{Disposables}

$0.5 \mathrm{~mL} / 1.5 \mathrm{~mL}$ microfuge tubes

$15 \mathrm{~mL}$ polystyrene tubes

$50 \mathrm{~mL}$ polystyrene tubes

$75 \mathrm{~cm}^{2}$ culture flasks

96- and 24-well low cell binding plates

96-well plates

Sodium-heparin $10 \mathrm{~mL}$ vacutainers

\section{Source}

\author{
Axygen, Union City, CA,USA \\ Falcon (BD Bioscience, NZ) \\ Falcon (BD Bioscience, NZ) \\ Falcon (BD Bioscience, NZ) \\ Nunc, Rochester, NY, USA \\ Falcon (BD Bioscience, NZ) \\ BD Bioscience, NZ
}

\subsection{Buffers and Media}

\section{Annexin-V binding buffer}

The efficient binding of annexin- $\mathrm{V}$ to phosphatidylserine on the surface of apoptotic cells requires a high calcium-containing buffer. This binding buffer was composed of 10mM HEPES, $140 \mathrm{mM} \mathrm{NaCl}$ and $2.5 \mathrm{mM} \mathrm{CaCl}_{2}$ at $\mathrm{pH}$ 7.4. The annexin- $\mathrm{V}$ binding buffer was filter sterilized and used fresh.

\section{FACS buffer}

FACS buffer, used as the staining media to label cell surface antigens with a fluorescently labeled cognate antibody. This solution contained 0.1\% Bovine serum albumin and $0.01 \%$ sodium azide in PBS (pH 7.4). 


\section{Hanks' balanced salt solution, HBSS}

There were two sources of HBSS used:

1. HBSS powder was purchased from Sigma-Aldrich, and made up to the appropriate volume with $\mathrm{ddH}_{2} \mathrm{O}$ and the $\mathrm{pH}$ adjusted to 7.4 according to the manufacturer's instructions, followed by filter sterilisation. This HBSS was used for procedures that did not require HBSS to be endotoxin free.

2. Endotoxin-tested HBSS was also purchased from Invitrogen in liquid form as Dulbecco's HBSS. This HBSS was used to wash and prepare cells that were going to be used in culture. (For composition refer to Appendix).

\section{Red Blood Cell (RBC) lysis buffer}

$\mathrm{RBC}$ lysis buffer was used to remove contaminating red blood cells from blood samples and peritoneal lavage fluids as required. Briefly, cell suspensions with contaminating RBCs were pelletted for 5 minutes at $500 \times g$ and the media carefully removed. 1 to $3 \mathrm{~mL}$ of RBC lysis buffer was added to the pellet and the cells gently resuspended by mild agitation. Cells were then incubated for 10 minutes at $37^{\circ} \mathrm{C}$, washed twice in HBSS and resuspended in the appropriate liquid medium. If RBC lysis was incomplete, the process was repeated.

Two lysis buffers were used.

1. Lysis buffer was prepared in the lab as two solutions: Solution $1\left(0.16 \mathrm{M} \mathrm{NH}_{4} \mathrm{Cl}\right)$ and Solution 2 (0.17M Tris). These buffers were filter sterilized and maintained under sterile conditions. A working RBC lysis buffer solution was prepared by mixing Solution 1 and Solution 2 at a 9:1 ratio immediately before use.

2. Endotoxin-free Puregene RBC lysis buffer was purchased from ProGENZ and was preferentially used instead of the in-house lysis buffer when the cells collected were used in further cell culture experiments. 
RPMI-1640

Endotoxin-tested RPMI-1640 was purchased in liquid form from Invitrogen. (For composition refer to Appendix). In all cases in which the term "complete RPMI-1640" appears, it refers to RPMI-1640 supplemented with 10\% FBS, 100U/mL penicillinstreptomycin and $2 \mathrm{mM}$ glutamax.

Phosphate buffered saline, PBS

There were two sources of PBS used:

1. PBS powder was purchased from Invitrogen, made up to the appropriate volume in $\mathrm{ddH}_{2} \mathrm{O}$ water and the $\mathrm{pH}$ adjusted to 7.4 according to the manufacturer's instructions, followed by filter sterilisation. This PBS was used for procedures that did not require PBS to be endotoxin free, such as for FACS buffer and saponin buffer solutions.

2. Endotoxin-tested PBS was also purchased from Invitrogen in liquid form as Dulbecco's PBS. This PBS was used to harvest and wash cells that were going to be used in culture. (For composition refer to Appendix).

\section{Saponin buffer}

Saponin is a detergent that creates pores within cell membranes making cells permeable to labeling antibodies and as such was used for staining intracellular cytokines with fluorescent antibodies. Saponin buffer was composed of $0.1 \%$ saponin, $0.1 \%$ bovine serum albumin and $0.01 \%$ sodium azide in PBS.

\section{Sodium phosphate buffer (50mM)}

Sodium phosphate buffer $(300 \mathrm{~mL})$ was prepared by mixing $65.8 \mathrm{~mL}$ of a $0.2 \mathrm{M} \mathrm{NaH}_{2} \mathrm{PO}_{4}$ solution with $9.2 \mathrm{~mL}$ of a $0.2 \mathrm{M} \mathrm{Na}_{2} \mathrm{HPO}_{4}$ solution. The mixture was made up to $300 \mathrm{~mL}$ with $\mathrm{ddH}_{2} \mathrm{O}$ and the $\mathrm{pH}$ adjusted to $\mathrm{pH}$. The solution was used immediately. 


\subsection{Preparation of MSU}

Monosodium urate crystals were prepared by crystallization of a supersaturated solution of uric acid under mildly basic conditions. Briefly, $250 \mathrm{mg}$ uric acid was added to $45 \mathrm{~mL}$ of $\mathrm{ddH}_{2} \mathrm{O}$ containing $300 \mu \mathrm{L}$ of $5 \mathrm{M} \mathrm{NaOH}$ and the solution boiled until the uric acid was dissolved. The solution was passed through a $0.2 \mu \mathrm{M}$ filter and $1 \mathrm{~mL}$ of $5 \mathrm{M} \mathrm{NaCl}$ was added to the hot solution that was then stored at $26^{\circ} \mathrm{C}$ to allow crystallisation. After 7 days the resulting MSU crystals were washed with ethanol and acetone over a Buchner flask and allowed to air dry under sterile conditions. Crystals showed typical physical characteristics of MSU, including a needle-like shape and optical birefringence. The typical crystal length was $5-20 \mu \mathrm{m}$ and MSU was confirmed as endotoxin-free by LAL assay $(<0.01 \mathrm{EU} / 10 \mathrm{mg})$.

\subsection{Endotoxin levels}

Endotoxin can activate innate immune cells and as such stringent efforts were taken to ensure that reagents and glassware used for cell culture were not only sterile but endotoxin free. All culture tubes, flasks, microplates and pipettes were explicitly pyrogen (endotoxin) free. Reagents used were either certified as low endotoxin or measured for endotoxin levels by LAL assay.

\subsubsection{Limulus amebocyte lysate (LAL) assay tested}

The following were tested for endotoxin levels by LAL assay. The assay kit was run according to manufacturer instructions

MSU crystals

MilliQ water $\left(\mathrm{ddH}_{2} \mathrm{O}\right)$

Cleaned and autoclaved glassware
$<0.01 \mathrm{EU} / 10 \mathrm{mg}$ $<0.01 \mathrm{EU} / \mathrm{mL}$ $<0.01 \mathrm{EU} / \mathrm{mL}$

(The inside of a $250 \mathrm{~mL}$ piece of glassware was rinsed with $10 \mathrm{~mL}$ of water provided in the LAL kit and the $10 \mathrm{~mL}$ of water was tested) 


\subsubsection{Purchased reagents}

$5 \mathrm{M} \mathrm{NaCl}$

D-PBS

HBSS

RPMI-1640

M199

Puregene RBC lysis buffer

Fetal bovine serum

$\begin{array}{ll}\text { Sigma-Aldrich, NZ } & <0.3 \mathrm{EU} / \mathrm{mL} \\ \text { Invitrogen, NZ } & <0.03 \mathrm{EU} / \mathrm{mL} \\ \text { Invitrogen, NZ } & <0.03 \mathrm{EU} / \mathrm{mL} \\ \text { Invitrogen, NZ } & <0.03 \mathrm{EU} / \mathrm{mL} \\ \text { Invitrogen, NZ } & <0.03 \mathrm{EU} / \mathrm{mL} \\ \text { ProGENZ, NZ } & <0.03 \mathrm{EU} / \mathrm{mL} \\ \text { Invitrogen, NZ } & <0.08 \mathrm{EU} / \mathrm{mL} \\ \text { Sigma-Aldrich, NZ } & <0.13 \mathrm{EU} / \mathrm{mL}\end{array}$

Sigma-Aldrich, NZ $<0.3 \mathrm{EU} / \mathrm{mL}$

Invitrogen, NZ $\quad<0.03 \mathrm{EU} / \mathrm{mL}$

Invitrogen, NZ $<0.03 \mathrm{EU} / \mathrm{mL}$

Invitrogen, NZ $\quad<0.03 \mathrm{EU} / \mathrm{mL}$

ProGENZ, NZ $\quad<0.03 \mathrm{EU} / \mathrm{mL}$

Sigma-Aldrich, NZ $<0.13 \mathrm{EU} / \mathrm{mL}$

\subsection{Isolation of cell types}

\subsubsection{Human neutrophils}

Neutrophils were purified from the peripheral blood of healthy volunteers by sedimentation with Polymorphprep. Briefly, human blood was collected into $10 \mathrm{~mL}$ Vacutainer tubes (anti-coagulated with heparin) by venous puncture and then diluted with an equal volume of HBSS. $10 \mathrm{~mL}$ of diluted blood was then carefully layered over $4 \mathrm{~mL}$ of Polymorphprep in a $15 \mathrm{~mL}$ tube and centrifuged with no brake at $500 \mathrm{x} g$ for 30 minutes at $19^{\circ} \mathrm{C}$. This treatment resulted in two distinct white bands: an upper band, containing mononuclear cells; and a lower band which contained polymorphonuclear cells. The polymorphonuclear cells were removed by aspiration and washed twice with an equal volume of PBS ( $\mathrm{pH} 7.4$ ), centrifuged at $500 \mathrm{x} g$ for 10 minutes at $19^{\circ} \mathrm{C}$ and then resuspended in the appropriate experiment buffer or medium. This procedure yielded $>90 \%$ neutrophils as determined by Diff-Quik staining of cytospin samples.

\subsubsection{Human peripheral blood mononuclear cells}

Peripheral blood mononuclear cells (PBMCs) were purified from the peripheral blood of healthy volunteers by sedimentation with Polymorphprep. Briefly, cells were prepared in the same way as neutrophils in 2.8.1 except the upper band of white blood cells was instead collected. PBMCs were $>95 \%$ pure (free of polymorphonucleocytes) by DiffQuik staining of cytospin samples. 


\subsubsection{Total human white blood cells}

Blood was collected from volunteers into heparinized Vacutainer tubes. The blood was then centrifuged at $500 \times \mathrm{g}$ for 10 minutes after which the layer of white blood cells (buffy coat) above the RBC layer was clearly visible and collected by aspiration. Contaminating red blood cells were lysed by treatment with RBC lysis buffer (Section 2.5), washed twice with HBSS and suspended in the appropriate medium for the relevant experiment.

\subsubsection{Enrichment of naïve mouse peritoneal lymphocytes and macrophages}

Peritoneal cells were harvested from naïve mice by peritoneal lavage as described (Section 2.16.3). Cells were retrieved from the lavage fluid by centrifugation ( $500 \times g, 5$ mins) and washed three times with PBS containing 10\% FCS and 10U/mL DNaseI. The cell types were then separated using a modified version of a method that has been previously described (DaMatta et al., 1995). Briefly, peritoneal leukocyte cells were resuspended in $70 \%(\mathrm{v} / \mathrm{v})$ Percoll solution containing DNaseI $(10 \mathrm{U} / \mathrm{mL})$ to a concentration of $2 \times 10^{6}$ cells $/ \mathrm{mL}$. The Percoll gradient was then prepared by overlaying the $70 \%$ Percoll-cell preparation $(3 \mathrm{~mL})$ with a $51 \%$ Percoll solution $(10 \mathrm{~mL})$, then overlaying Medium $199(2 \mathrm{~mL})$ containing DNaseI $(10 \mathrm{U} / \mathrm{mL})$ as the uppermost layer. The gradient preparation was centrifuged with no brake $\left(18 \mathrm{mins}, 680 \times \mathrm{g}, 4^{\circ} \mathrm{C}\right)$ and macrophages recovered from the $8-15 \mathrm{~mL}$ band (purity greater than $90 \%$, viability greater than 98\%), while lymphocytes were recovered from the $4.5-8 \mathrm{~mL}$ band (purity greater than $80 \%$, viability greater than $90 \%$ ). Cells were then washed twice in HBSS containing DNaseI $(10 \mathrm{U} / \mathrm{mL})$ and resuspended in complete RPMI-1640 to give a final concentration of $1 \times 10^{6}$ cells $/ \mathrm{mL}$. 


\subsubsection{Enrichment of peritoneal mast cells}

Peritoneal cells were harvested from naïve mice by peritoneal lavage as described (Section 2.16.3). Cells were retrieved from the lavage fluid by centrifugation $(500 \times g, 5$ mins) and washed three times with PBS containing 10\% FCS and 10U/mL DNaseI. The cells were then resuspended in $8 \mathrm{~mL}$ of $70 \%$ Percoll solution $(6.3 \mathrm{~mL}$ Percoll Plus, 3.7 $\mathrm{mL}$ of $2.5 \mathrm{x}$ PBS, $\mathrm{pH} 7.2$ ) and overlayed with $2 \mathrm{~mL}$ of RPMI-1640 (5\% FBS). The gradient was centrifuged with no brake (15 min, $500 \mathrm{x} \mathrm{g}$, room temperature), the Percoll solution was removed and the cell pellet was washed in complete RPMI-1640. The cells were re-suspended in complete RPMI-1640 to give a final concentration of $1 \times 10^{5}$ mast cells/mL. Purity was greater than $95 \%$ while viability was at least $98 \%$ as determined by Trypan Blue.

\subsubsection{Sorting of murine leukocyte populations}

Antibodies against F4/80 and Gr-1, and the antibody clone 7/4 were used to discriminate macrophages, monocytes and neutrophils as previously described (Austyn and Gordon, 1981, Henderson et al., 2003). Mice were administered with an i.p. injection of MSU (3mg, 0.5mL PBS) and at different time points cells were harvested as described (Section 2.16.3) and stained with F4/80, Gr-1 and 7/4 antibodies. Resident macrophages were identified from naïve mice as $\mathrm{F} 4 / 80^{\mathrm{hi}}$, Gr-1 ${ }^{-}$, 7/4 ${ }^{-}$cells; monocytes were identified from mice 4 or 8 hours after MSU treatment as F4/80 $0^{\mathrm{lo}}$, Gr- $1^{\text {int }}, 7 / 4^{+}$cells; neutrophils were identified from mice 8 hours after MSU treatment as F4/80- Gr-1 ${ }^{\text {hi }}, 7 / 4^{+}$cells; and immature macrophages were identified 48 hours after MSU treatment as F4/80 ${ }^{\text {lo }}, \mathrm{Gr}-1^{-}$, 7/4 cells. Cells were sorted using a FACSDiva flow cytometer and determined to be $>99 \%$ pure by flow cytometry and Diff-Quik staining.

\subsection{Cell lines and culture}

\subsubsection{Human umbilical vein endothelial cells (HUVEC)}

HUVEC cells are primary cells harvested from the umbilical cord of newborn human babies and are endothelial/epithelial in nature. HUVECs were harvested and prepared to passage three by Dr. Sarah Gunningham at the (Christchurch School of Medicine and 
Health Science, University of Otago) and sent to the Malaghan Institute of Medical Research (Victoria University of Wellington). HUVECs were re-suspended in Medium 199 (supplemented with 10\% FBS, $1 \mathrm{ng} / \mathrm{mL}$ bFGF) and cultured in $75 \mathrm{~cm}^{2}$ culture flasks coated with $0.1 \%$ gelatin, seeding the flask with $2 \times 10^{5}$ cells. Cells were grown at $37^{\circ} \mathrm{C}$ and $5 \% \mathrm{CO}_{2}$ until just before confluent. The HUVEC monolayer was then washed twice with HBSS and $10 \mathrm{~mL}$ of $0.25 \%$ trypsin/EDTA was added. The HUVEC culture was then returned to the incubator $\left(37^{\circ} \mathrm{C}, 5 \% \mathrm{CO}_{2}\right)$ for 5 minutes, after which HUVEC detachment could be observed by microscopic examination. 10mL of FBS was added to inactivate the trypsin enzyme activity and then cells were collected into a $50 \mathrm{~mL}$ Falcon tube. HUVECs were washed twice in HBSS, re-suspended in Medium 199 (supplemented with $10 \% \mathrm{FBS}, 1 \mathrm{ng} / \mathrm{mL}$ bFGF) and then split into three separate flasks after which cells were allowed to grow to confluence again. HUVECs were cultured in this way to passage 7 and then used experimentally.

\subsubsection{RAW 264.7 cells}

Murine macrophage cell line RAW 264.7 were seeded at $2 \times 10^{5}$ cells $/ 75 \mathrm{~cm}^{2}$ culture flask in complete RPMI-1640. Cells were grown to confluence at $37^{\circ} \mathrm{C}$ and $5 \% \mathrm{CO}_{2}$ after which the cell monolayer was washed with HBSS and then harvested using a rubber scraper. Cells were then washed again and resuspended in complete RPMI-1640 at a cell concentration of $1 \times 10^{6}$ cells $/ \mathrm{mL}$ and plated out into 96 well plates at $200 \mu \mathrm{L}$ per well for the subsequent experiment.

\subsubsection{Culture of primary mouse mesothelial cells}

Mouse mesothelial cells were harvested and grown based on previously described methods(Bot et al., 2003, Muller and Yoshida, 1995). C57/BL6 mice were euthanised by $\mathrm{CO}_{2}$ and the mice sprayed liberally with $70 \%$ ethanol and placed into a sterile safety hood. The skin and fur covering the peritoneum were carefully separated without puncturing the peritoneal lining. This skinning technique allowed clean exposure of the peritoneal tissue without puncturing the peritoneal cavity and completely preventing fur from coming into contact with the peritoneal tissue. Peritoneal leukocytes were removed by injecting $10 \mathrm{~mL}$ of PBS into the peritoneal cavity, injecting near the inguinal 
fat pads to allow the resealing of the injection site. After gently massaging the inflated peritoneum, the PBS (containing the resident leukocytes) was removed, and the whole lavage process repeated. Next, $10 \mathrm{~mL}$ of $0.25 \%$ trypsin/EDTA warmed to $37^{\circ} \mathrm{C}$ was injected into the peritoneum and allowed to sit for 15 minutes under a heat lamp with occasional massaging. The outside of the peritoneum was kept moist with PBS. The trypsin/EDTA was then harvested and washed twice in complete RPMI-1640. The harvested cells were then resuspended in RPMI-1640 containing 20ng/mL epithelial growth factor and $5 \mu \mathrm{M}$ hydrocortisone and cultured in $25 \mathrm{~cm}^{2}$ collagen-coated flasks. Cells were cultured and split three times before use in experiments and were confirmed as mesothelial cells by their squamous cobblestone appearance and positive staining for cytokeratin.

\subsection{Histology}

\subsubsection{Morphological stain for differential cell counts}

Diff-Quik staining was used to visualize the morphology of white blood cells harvested from blood or peritoneal lavage samples allowing differential counts. Cells were placed onto slides by a cytospin cytocentrifuge and were air-dried, fixed for 5 seconds in DiffQuik fixative (1.8mg/L Triarylmethane dye methyl alcohol) stained for 10 seconds in Diff-Quik Solution I (1g/L xanthine dye) and then stained for 7 seconds in Diff-Quik Solution II (0.625g/L Azure A, 0.625g/L Methylene blue). Alternatively, cells were fixed for 20 minutes in 10\% buffered formalin, stained for 10 seconds in Diff-Quik Solution I, followed by a 4 second staining with Diff-Quik Solution II. Excess dye was then washed off with water. For differential cell counts, 200 cells were counted per sample.

\subsubsection{Hematoxylin and Eosin $(H \& E)$ staining of peritoneal tissue}

Mice were administered with an i.p. injection of MSU (3mg; 0.5mL PBS) and 72 hours after treatment, peritoneal membrane tissue was excised and placed into $10 \%$ neutral buffered formalin. Peritoneal membrane tissue was also taken from naïve mice. Tissue 
cross sectioning and H\&E staining was then performed at the Department of Pathology at the Wellington School of Medicine, NZ.

\subsubsection{Visualisation of MSU crystal and cell clumping}

Clumping of MSU crystals with cells was observed in two ways. In the first method, clumps of MSU crystals were harvested from the peritonea of MSU-treated mice. Clumps were placed into suspension in PBS and then sedimented onto slides using a cytocentrifuge. The mesh of crystals and cells were stained with Diff-Quik after which they were examined using an Olympus BX51 microscope under bright field and polarized light conditions. In the second method, peritoneal leukocytes $\left(1 \mathrm{x} 10^{6}\right.$ cells $/ \mathrm{mL}, 200 \mu \mathrm{L}$ ) from naïve mice were cultured in 8-well chamber slides for one hour following which $200 \mu \mathrm{g} / \mathrm{mL}$ MSU was added to the wells. After 30 minutes, the slides were carefully washed with PBS and the cells fixed with zinc fixative. After 20 minutes, slides were carefully washed three times with PBS after which they were stained with Alexa 488-anti-F4/80 and Hoechst 33342 in staining buffer (5\% FBS, 0.01\% sodium azide, PBS; pH 7.4). Cells were then examined by fluorescent microscopy.

\subsubsection{Immunofluorescent histology of resident peritoneal macrophages}

Resident peritoneal cells from naïve mice were suspended in complete RPMI-1640 and cultured at $1 \times 10^{6}$ cells $/ \mathrm{mL}$ in 8 -well chamber slides. After incubation at $37^{\circ} \mathrm{C}$ for 1 hour, 1:1500 GolgiStop was added. Cells were stimulated with either 200 $\mathrm{g} / \mathrm{mL} \mathrm{MSU}$, $1 \mu \mathrm{g} / \mathrm{mL}$ LPS, or PBS and incubated for 4 hours, then treated with zinc fixative for 30 minutes. Saponin buffer was used to permeabilise the cells and then endogenous biotin blocked using a biotin blocking kit as per manufacturer's instructions. Non-specific IgG binding sites were then blocked using 5\% mouse serum or 5\% FBS and incubated overnight with either anti-mouse IL-6, TNF $\alpha$, biotinylated anti-mouse IL-1 $\beta$, or the appropriate isotype control. Cells treated with anti-mouse IL-6 or TNF $\alpha$ were then incubated with a biotinylated mouse-anti-rat IgG antibody. Excess antibody was removed by washing with PBS and the samples stained with streptavidin-Alexa555, anti-F4/80-FITC and Hoechst 33342, mounted in Vectashield antifade and examined using an Olympus BX51 fluorescence microscope. 


\subsubsection{Staining of visceral peritoneal tissue}

Mice were treated with an i.p. injection of $3 \mathrm{mg}$ MSU as described above. After 4 hours the skin was removed from the abdomen, the peritoneum was cleared of leukocytes by lavage with $3 \mathrm{~mL}$ PBS and the ventral tissue covering the peritoneal cavity was excised and placed into cold (incomplete) RPMI-1640. The tissue was rinsed in PBS and fixed in acetone for 10 minutes at $-20^{\circ} \mathrm{C}$. The visceral lining of the peritoneum was harvested from the fixed tissue by carefully stripping the lining off as a single piece. The harvested visceral tissue was cleaned of fascia and non-specific IgG binding sites were blocked with a solution containing 10\% FBS. Endogenous biotin was blocked using a kit. The tissue was stained for the surface markers 7/4 and F4/80 and mounted onto slides with Vectashield antifade. Slides were analysed by fluorescence microscopy and images processed using AnalySIS Life Science extended focal imaging software.

\subsection{Measurement of superoxide production}

Measurement of neutrophil superoxide production was performed using using the tetrazolium salt, WST-1. WST-1 is commonly used in the presence of 1-methoxy-5methylphenazinium methylsulfate (PMS) to measure cell proliferation. However, Tan and Berridge showed that in the absence of PMS, WST-1 reduction is indicative of superoxide production in neutrophils (Tan and Berridge, 2000).

\subsubsection{PMA stimulation of neutrophils}

Purified human neutrophils were suspended in the appropriate medium condition (PBS, HBSS, phenol red-free RPMI-1640 supplemented 100U/mL penicillin-streptomycin, $2 \mathrm{mM}$ glutamax, \pm human serum) and added to a 96 well plates at $1 \times 10^{5}$ neutrophils per well with $250 \mu \mathrm{g} / \mathrm{mL}$ WST-1 and stimulated with $0.2 \mu \mathrm{g} / \mathrm{mL}$ PMA in a final total volume of $100 \mu \mathrm{L}$ per well. Immediately following PMA addition, the plate was loaded into a Versamax spectrophotometer preheated and maintained at $37^{\circ} \mathrm{C}$ and the maximum rate of change of absorbance $\left(\mathrm{V}_{\max }\right)$ at $450 \mathrm{~nm}$ measured over 20 minutes. For experiments with the sesquiterpene dialdehyde compounds, the compounds were added 30 minutes prior to stimulation with PMA. 


\subsubsection{MSU stimulation of neutrophils}

Purified human neutrophils were suspended in HBSS or the relevant conditioned media and placed into $0.5 \mathrm{~mL}$ microfuge tubes at $1.5 \times 10^{5}$ neutrophils per tube in a total volume of $140 \mu \mathrm{L}$ per well. If neutrophils were being cultured in conditioned media, then neutrophils were subsequently incubated for 2 hours at $37^{\circ} \mathrm{C}$ and $5 \% \mathrm{CO}_{2}$ to allow the neutrophils to adjust to the media condition. For neutrophils in HBSS, this incubation step was not required. WST-1 $(250 \mu \mathrm{g} / \mathrm{mL})$ and a range of concentrations of MSU in either HBSS or phenol red-free RPMI-1640 (depending on whether HBSS or RPMI-1640 was the neutrophil culture media) were then added to a final total volume of $150 \mu \mathrm{L}$. Tubes were then capped and placed into an incubator on a shaker $(200 \mathrm{rpm}$, $15 \mathrm{~mm}$ radius) at $37^{\circ} \mathrm{C}$. After 1 hour MSU crystals were removed from the supernatant by centrifugation and $100 \mu \mathrm{L}$ of the crystal-free supernatant was transferred to a 96 well plate. The plate was then loaded into a Versamax spectrophotometer and the absorbance measured at $450 \mathrm{~nm}$.

\subsubsection{Serum stimulation of neutrophils}

Purified human neutrophils were suspended in various concentrations (0 to 10\%) of human serum or FBS, suspending in phenol-red free RPMI-1640. The effect of autologous versus the use of non-autologous human serum was tested as part of specific experiments and is outlined in chapter 3 (Section 3.2.2); otherwise, non autologous human serum was used. Human and bovine serum that had been heat inactivated at $56^{\circ} \mathrm{C}$ for 30 minutes was also tested. $1.5 \times 10^{5}$ neutrophils were added to $0.5 \mathrm{~mL}$ microfuge tubes, following which, WST-1 $(250 \mu \mathrm{g} / \mathrm{mL})$ was then added to the tubes to a final total volume of $150 \mu \mathrm{L}$. Tubes were then capped and placed into a shaking incubator $(200 \mathrm{rpm}, 15 \mathrm{~mm}$ radius $)$ at $37^{\circ} \mathrm{C}$. After 1 hour cells were removed from the supernatant by centrifugation and $100 \mu \mathrm{L}$ of the supernatant was transferred to a 96 well plate. The plate was then loaded into a Versamax spectrophotometer and the absorbance measured at $450 \mathrm{~nm}$ 


\subsubsection{Superoxide production by ex vivo murine peritoneal neutrophils}

Mice received an i.p. injection of the sesquiterpene dialdehyde compound in $50 \mu \mathrm{L}$ of DMSO or $50 \mu \mathrm{L}$ of DMSO alone (control) to one side of the peritoneum following which an i.p. injection of $\mathrm{MSU}(3 \mathrm{mg}, 0.5 \mathrm{~mL}$ PBS) was immediately administered to the opposite side of the peritoneum as described (Section 2.16.3). After four hours, peritoneal leukocytes were harvested by peritoneal lavage with $3 \mathrm{~mL}$ PBS containing $25 \mathrm{U} / \mathrm{mL}$ heparin. Cells were then washed and the concentration adjusted to $1 \times 10^{6}$ cells $/ \mathrm{mL}$ in HBSS. $100 \mu \mathrm{L}$ of the cell suspension was added to 96-well plates without the addition of any stimulus ex vivo, following which WST-1 added $(250 \mu \mathrm{g} / \mathrm{mL})$. Cells from a naïve mouse, which do not contain neutrophils, were also included as a negative control. The plate was then loaded into a Versamax spectrophotometer that had been preheated and maintained at $37^{\circ} \mathrm{C}$ and the maximum rate of change of absorbance at 450nm measured over 20 minutes. Cytospin samples of each cell suspension were also prepared and differential counts obtained by Diff-Quik staining. $\mathrm{V}_{\max }$ values obtained from the Versamax readings were then adjusted for neutrophil percentages to give a $\mathrm{V}_{\max }$ value per $1 \times 10^{6}$ cells. For mice treated with sesquiterpene dialdehyde compounds, the final $\mathrm{V}_{\max }$ value was normalized to the Vmax of cells from mice that had been treated with the control carrier solvent.

\subsubsection{Luminol chemiluminescence}

The measurement of NADPH oxidase generated ROS from HUVECs was measured using peroxidase catalyzed luminol chemiluminescence. HUVECs suspended in phenol red-free Medium 199 containing 0.5\% FBS (or higher \% FBS for serum studies) were plated to a 96-well white plate at $2 \times 10^{5}$ cells/well. Sesquiterpene dialdehyde compounds, DPI, L-NAME or controls were added to the cells and the cells incubated for 1 hour at $37^{\circ} \mathrm{C}$ and $5 \% \mathrm{CO}_{2} . \quad 10 \mathrm{U} / \mathrm{mL}$ horseradish peroxidase and $10 \mu \mathrm{M}$ luminol were added to the cells with a final volume of $200 \mu \mathrm{L} /$ well. The plate was then placed in a $\mathrm{BMG}$ fluostar microplate reader that had been pre-warmed to $37^{\circ} \mathrm{C}$. The total emitting light was measured over 1 hour without any emission filter and the resulting $\mathrm{V}_{\max }$ values (Units/sec) normalized to the DMSO controls. 


\subsection{Viability stains}

\subsubsection{Trypan blue}

Cell membranes are impermeable to the dye trypan blue. Once cells have died and lost cell membrane integrity trypan blue can then be taken up by cells that then become blue in colour. Cells were diluted 1:1 and then enumerated using a hemocytometer. The cell concentration was determined by taking the number of cells in a quadrant and multiplying by $2 \times 10^{4}$ to give cells $/ \mathrm{mL}$. Alternatively cells were diluted 1:9 (cell suspension:Trypan blue dye) and multiplied by $1 \times 10^{5}$ to give cells $/ \mathrm{mL}$. Cells were not in trypan blue suspension for more than 5 minutes to reduce the possibility of false positives.

\subsubsection{Neutrophil trypan blue assay for sesquiterpene dialdehyde compounds}

Purified human neutrophils were suspended in HBSS and cultured in 24 well plates at a concentration of $1 \times 10^{6}$ cells $/ \mathrm{mL}$ and at a volume of $500 \mu \mathrm{L}$ per well. Sesquiterpene dialdehyde compounds or DMSO controls were then added. After 30 minutes, a sample of each well was diluted in an equal volume of trypan blue, then the total live cells (clear in appearance) and dead cells (blue in appearance) enumerated using a hemocytometer. 200 cells were counted.

\subsubsection{Annexin-V and propidium iodide (PI) double staining}

Cellular apoptosis is associated with a number of cellular changes, one of which is the externalization of phosphatidylserine (PS) from the inner leaflet of the plasma membrane to the outer leaflet. The exposure of PS makes it available for binding to annexin-V, a calcium-dependent phospholipids-binding protein that has a high affinity for PS. Because of this, a fluorescently labeled annexin-V protein can be used to determine the presence of apoptotic cells in the presence of a high calcium-containing buffer (Section 2.5) (Vermes et al., 1995). However, both necrotic and late stage apoptotic cells can appear as annexin- $\mathrm{V}^{+}$as a loss of cell integrity allows annexin- $\mathrm{V}$ to penetrate and stain intracellular PS. To make the distinction of the different modes of cell death, PI is also used as a viability stain. PI binds to DNA but only when a cell membrane has lost integrity and become permeable (Nicoletti et al., 1991). When 
observed over time, changes in annexin-V and PI staining can be indicative of the process of cell death. Double negatives indicate viable cells, while annexin-V single positives indicate early apoptotic cells. PI single positives are necrotic cells while annexin-V/PI double positives are late stage dying and dead cells. Hence, annexin-V/PI staining not only indicates the proportion of cells undergoing each different stage of viability/cell death, but also the mode of cell death depending on whether the cells first go through an annexin-V single positive stage, or a PI single positive stage.

\subsubsection{Measuring the effect of sesquiterpene dialdehyde compounds on neutrophil viability by annexin-V/PI staining}

Purified human neutrophils were suspended in complete RPMI-1640 and cultured in 24 well plates at a concentration of $1 \times 10^{6}$ cells $/ \mathrm{mL}$ and at a volume of $500 \mu \mathrm{L}$ per well. Sesquiterpene dialdehyde compounds (ranging from 0.01 to $10 \mu \mathrm{M}$ in concentration) or DMSO controls were then added. After four hours, neutrophils were collected and washed and stained with FITC labeled annexin-V $(5 \mu \mathrm{L} / 100 \mu \mathrm{L}$ cells $)$ and $10 \mu \mathrm{g} / \mathrm{mL}$ PI in annexin-V binding buffer. Staining was then determined by flow cytometry and viability determined as described in Section 2.12.3.

\subsection{Cytokine analyses}

Cytokine levels in harvested lavage fluid, serum and from culture supernatants were assayed either by Cytokine Bead Array (CBA) and analysed on a FACSCalibur flow cytometer, by Bio-Rad multiplex array systems and analysed on a Bio-Plex flow cytometer, or by ELISA. Each measurement system was used according to manufacturer's instructions. 


\subsection{Human neutrophil, mononuclear cell and white blood cell experiments}

\subsubsection{Neutrophil and WBC apoptosis and viability assay}

Fresh human neutrophils or WBCs were suspended in complete RPMI-1640 and were added to 96 well plates at $2 \times 10^{5}$ cells/well in the presence of MSU $(200 \mu \mathrm{g} / \mathrm{mL})$ or PBS $(10 \mu \mathrm{L})$ in a final total volume of $200 \mu \mathrm{L}$. Neutrophils were then incubated at $37^{\circ} \mathrm{C}$ and $5 \% \mathrm{CO}_{2}$. At various times, neutrophils were harvested and stained immediately with FITC labeled annexin-V $(5 \mu \mathrm{L} / 100 \mu \mathrm{L}$ cells $)$ in annexin-V binding buffer. Anti human CD15 antibody was also included in the staining mix. CD15 is a carbohydrate adhesion molecule with a high expression on neutrophils, thus anti CD15 can be used to identify neutrophils in mixed WBC populations (Kerr and Stocks, 1992). Five minutes before analysis on the flow cytometer, $10 \mu \mathrm{g} / \mathrm{mL}$ PI was added and then annexin-V/PI staining determined using a FACSCalibur flow cytometer. When testing for the effect of sesquiterpene dialdehyde compounds on cell viability, sesquiterpene dialdehyde compounds were added at time $0 \mathrm{~h}$ and the neutrophils and $\mathrm{WBC}$ recovered and stained with annexin-V $(5 \mu \mathrm{L} / 100 \mu \mathrm{L}$ cells $), 10 \mu \mathrm{g} / \mathrm{mL}$ PI and anti-CD15 in annexin-V binding buffer, 4 hours and 24 hours after incubation.

\subsubsection{Neutrophil and WBC soluble cytokine production}

Fresh human neutrophils or WBCs were suspended in complete RPMI-1640 and were added to 96 well plates at $2 \times 10^{5}$ cells/well in the presence of MSU $(200 \mu \mathrm{g} / \mathrm{mL})$ or PBS $(10 \mu \mathrm{L})$ in a final total volume of $200 \mu \mathrm{L}$. Neutrophils were then incubated at $37^{\circ} \mathrm{C}$ and $5 \% \mathrm{CO}_{2}$ and at various times the supernatants were collected and the cytokines in the serum assayed by CBA array multiplex kit.

\subsubsection{Preparation of conditioned media}

Purified human PBMCs were suspended at $5 \times 10^{5}$ cells $/ \mathrm{mL}$ in phenol red free RPMI1640 supplemented with $10 \%$ autologous human serum, $100 \mathrm{U} / \mathrm{mL}$ penicillinstreptomycin and $2 \mathrm{mM}$ glutamax. $40 \mathrm{~mL}$ volumes of the cell suspension were then transferred to $75 \mathrm{~cm}^{2}$ cell culture flasks. MSU crystals $(200 \mu \mathrm{g} / \mathrm{mL})$ were then added to the cell culture flasks to prepare "MSU-conditioned media," while PBS $(500 \mu \mathrm{L})$ was 
added to flasks to prepare "conditioned media." The cell cultures were then incubated for 16 hours at $37^{\circ} \mathrm{C}, 5 \% \mathrm{CO}_{2}$. A $40 \mathrm{~mL}$ volume of the same RPMI-1640 media, which contained no cells, was also transferred into a $75 \mathrm{~cm}^{2}$ cell culture flask and incubated with the other cell cultures overnight to serve as a negative control that was termed "unconditioned media." After incubation, the supernatants were collected and all MSU crystals and cells removed by centrifugation. Supernatants were then stored at $-20^{\circ} \mathrm{C}$ until analysis.

\subsubsection{Intracellular IL-8 staining of neutrophils}

Human neutrophils were suspended in the various conditioned media in 24 well low cell binding plates at a concentration of $1 \times 10^{6}$ cells $/ \mathrm{mL}$ in a total volume of $500 \mu \mathrm{L}$. MSU $(200 \mu \mathrm{g} / \mathrm{mL})$ or PBS $(20 \mu \mathrm{L})$ was added and neutrophils incubated for 4 hours at $37^{\circ} \mathrm{C}$ and $5 \% \mathrm{CO}_{2}$. Neutrophils were then fixed in $10 \%$ buffered formalin for 15 minutes, permeabilised with saponin buffer and then stained with fluorescently labeled anti human IL-8 or an Isotype control. Saponin buffer and excess antibody was then washed off by washing three times with FACS buffer. The percentage of IL- $8^{+}$neutrophils suspended in FACS buffer was then determined by flow cytometry.

\subsubsection{Measuring the effect of soluble mediators on neutrophil viability}

Human neutrophils were suspended in the various conditioned media in 24 well low cell binding plates at a concentration of $1 \times 10^{6}$ cells $/ \mathrm{mL}$ in a total volume of $500 \mu \mathrm{L}$ and incubated for 24 hours at $37^{\circ} \mathrm{C}$ and $5 \% \mathrm{CO}_{2}$. Neutrophils were collected and washed and stained with fluorescently labeled annexin-V and PI in annexin-V binding buffer. Staining was then determined by flow cytometry and viability determined as described in Section 2.12.3.

\subsubsection{Measuring the effect of soluble mediators on MSU-stimulated superoxide production}

This was performed as outlined in Section 2.11.2. 


\subsection{Phagocytosis assays}

\subsubsection{Phagocytosis of fluorescent beads}

Peritoneal exudates cells were harvested from MSU treated mice at various times after MSU administration as described (Section 2.16.3). Cells were suspended at $1 \times 10^{6} / \mathrm{mL}$ in RPMI-1640 with $10 \%$ FBS and $2 \mathrm{~mL}$ of the cell suspension placed in $10 \mathrm{~mL}$ falcon tube. Fluorescent beads (fluospheres, 2\% solids) were separately diluted in PBS, and $50 \mu \mathrm{L}$ of diluted beads added to the cells to the give the final dilution factor. That is, a dilution factor of $5 \times 10^{-4}$ represented that beads in the $2 \mathrm{~mL}$ cell suspension were 50,000 times more dilute than in the original purchased beads stock solution ( $2 \%$ solids). The bead and cell suspension was then placed on a shaker in an incubator $(200 \mathrm{rpm}, 15 \mathrm{~mm}$ radius) at $37^{\circ} \mathrm{C}$ for 30 minutes. Cells were then layered over the top of $1 \mathrm{~mL}$ of FBS and the suspension centrifuged for 5 minutes at $500 \times g$. This procedure pelleted the cells while unphagocytosed beads remained at the serum-medium interface. The supernatant was then removed and the cells washed twice in FACS buffer, stained for the appropriate cell surface markers and analysed by flow cytometry.

\subsubsection{Peroxidase staining for phagocytosed neutrophils}

Peritoneal exudate cells were harvested from MSU treated mice at various times after MSU administration as described (Section 2.16.3). Cells were suspended at $1 \times 10^{6}$ cells/mL in complete RPMI-1640 and split into two groups, the first of which was processed immediately following cell harvest. The second group was placed in a shaking incubator (160 rpm, $15 \mathrm{~mm}$ radius) and $37^{\circ} \mathrm{C}$ in $50 \mathrm{~mL}$ Falcon tubes to allow for the

phagocytosis of apoptotic neutrophils for 4,18 or 22 hours (depending on the experiment), after which the cells were processed in the same way as the previous group. Harvested cells were washed and re-suspended in PBS and samples of the cells prepared by cytospin. The cells were then fixed with $10 \%$ buffered formalin for 20 minutes at $37^{\circ} \mathrm{C}$ and washed twice with PBS. Cells were then stained with peroxidase stain $(1 \mathrm{mM}$ $o$-dianisidine, $5 \mathrm{mM} \mathrm{H}_{2} \mathrm{O}_{2}, 50 \mathrm{mM}$ sodium phosphate buffer, $\mathrm{pH}$ 6) for 30 minutes at $37^{\circ} \mathrm{C}$ and then washed twice more in PBS followed by a counter stain with Diff-Quik. Monocyte-macrophages were then scored for their level of peroxidase staining in the cytoplasm based on a three tier scale: Negative, containing no staining; Low, containing 
a few speckled traces of staining in the cytoplasm; and High, containing easily visible phagocytosed cells or extensive staining through the cytoplasm.

\subsection{Animal studies}

\subsubsection{Ethical approvals}

All experimental procedures were approved by the Victoria University Animal Ethics Committee in accordance with their guidelines for the care of animals.

\subsubsection{Mice}

C57BL/6 male mice were bred and housed in a conventional animal facility at the Malaghan Institute of Medical Research, Wellington, New Zealand. All animals used for experiments were aged between 8-10 weeks.

\subsubsection{MSU-induced peritonitis}

C57BL/6 mice were administered with an i.p. injection of a $3 \mathrm{mg}$ slurry of MSU in $0.5 \mathrm{~mL}$ PBS. At different time points mice were euthanased by $\mathrm{CO}_{2}$ and the blood harvested by cardiac puncture. Mice were then dowsed with $70 \%$ ethanol and the fur covering the peritoneum carefully removed without puncturing the peritoneal membrane. A $3 \mathrm{~mL}$ volume of PBS containing $25 \mathrm{U} / \mathrm{mL}$ heparin was then injected into the peritoneum, injecting near the inguinal fat pads to allow the injection hole to re-seal on extraction of the syringe needle. The inflated peritoneum was then massaged for 30 seconds following which the syringe needle was re-introduced into the peritoneum and the PBS (containing peritoneal exudate cells) were withdrawn. The peritoneal lavage fluid was expelled into a sterile tube containing $200 \mu \mathrm{L}$ FBS, unless the lavage fluid was to be measured for TGF $\beta$ in which case FBS was excluded. Serum and lavage fluid was

retained for cytokine analysis. Cells were retrieved from the lavage fluid by centrifugation (500 x $g, 5$ mins) and were subsequently washed twice with PBS before further culture or before antibody staining for analysis by flow cytometry. Cytospin samples of the cells were prepared by sedimentation using a cytocentrifuge, after which they were stained with Diff-Quik. For differential counts using these samples, 200 cells were examined per slide. 


\subsubsection{Thioglycollate-induced peritonitis}

C57BL/6 mice were administered with an intraperitoneal (i.p.) injection of $4 \%$ thioglycollate $(0.5 \mathrm{~mL})$. After six hours the mice were euthanased by $\mathrm{CO}_{2}$ and the peritoneal exudate cells harvested as described for MSU crystal-induced peritonitis above.

\subsubsection{Testing of sesquiterpene dialdehyde compounds in the in vivo gout model}

Mice were treated with a $50 \mu \mathrm{L}$ i.p. injection of the sesquiterpene dialdehyde compound or $50 \mu \mathrm{L}$ of DMSO (control) to one side of the peritoneum following which an i.p. injection of MSU (3mg, 0.5mL PBS) was immediately administered to the opposite side of the peritoneum. Peritoneal leukocyte cells were harvested 4 hours after MSU administration by lavage with $3 \mathrm{~mL}$ of PBS containing $25 \mathrm{U} / \mathrm{mL}$ heparin and $10 \% \mathrm{FBS}$. In addition to the required cell number and differential counts, peritoneal leukocytes were retained to measure the levels of superoxide being produced by infiltrating neutrophils (Section 2.11.4).

\subsubsection{PKH26 experiments}

PKH26-PCL is a dye that is selectively taken up phagocytes; principally macrophages, and to a lesser extent, monocytes and neutrophils (Bellingan et al., 1996). PKH26-PCL dye was prepared according to manufacturer's instructions. Mice received an injection of $0.5 \mathrm{~mL}$ of the PKH26 dye solution i.p. and one day later the mice received an injection of MSU i.p. (3mg, 0.5mL PBS). Mice were sacrificed at various time points after MSU treatment and the peritoneal exudates cells harvested by peritoneal lavage as described (Section 2.16.3). Cells were then stained for Gr-1 and F4/80 and analysed by flow cytometry.

\subsubsection{Preparation of clodronate-loaded liposomes}

Clodronate liposomes were prepared as previously described (van Rooijen et al., 1996). Briefly, $86 \mathrm{mg}$ phosphatidylcholine and $8 \mathrm{mg}$ of cholesterol were dissolved in $10 \mathrm{~mL}$ of chloroform and added to a round-bottom flask. The flask was placed onto a rotary evaporator at high vacuum to form a thin film on the inside of the flask. $10 \mathrm{~mL}$ of $0.7 \mathrm{M}$ 
clodronate ( $\mathrm{pH}$ 7.1) was added and then the film gently dispersed by gentle rotation for 10 minutes. The flask was then removed from the rotary evaporator, flushed with nitrogen and incubated overnight at $4{ }^{\circ} \mathrm{C}$ (still under nitrogen). The resulting liposomes were then harvested by centrifugation $2000 \times \mathrm{g}$ for 40 minutes, washed twice and made up to $4 \mathrm{~mL}$ in PBS. Liposomes were stored at $4^{\circ} \mathrm{C}$ and used within three days of production.

\subsubsection{Clodronate liposome depletion of resident macrophages}

Mice were treated with $200 \mu \mathrm{L}$ i.p. injection of either clodronate liposomes, PBS loaded liposomes or PBS. Three days following the first injection, mice were challenged with an i.p. injection of $3 \mathrm{mg}$ of MSU following which cells were harvested as described by lavage as above (Section 2.16.3).

\subsection{Intracellular staining of ex vivo cells}

Total peritoneal exudate cells from mice treated with MSU for 4 hours were harvested by peritoneal lavage and quickly suspended at $1 \times 10^{6}$ cells $/ \mathrm{mL}$ in complete RPMI-1640 and 1:1500 GolgiStop and transferred into 24 well low cell binding plates. Positive controls were stimulated with $1 \mu \mathrm{g} / \mathrm{mL}$ LPS after which all cells were incubated for a further 4 hours at $37^{\circ} \mathrm{C}$ to allow for accumulation of intracellular cytokine levels. Cells were then stained for the surface markers F4/80, Gr-1 and 7/4 and intracellular IL-6. Cells were analysed by flow cytometry using a BD FACSCalibur. Cytospin samples of exudate cells were also prepared by Diff-Quik staining to complement differential cell counts determined by flow cytometry.

\subsection{Restimulation assays}

Harvested peritoneal exudate cells from MSU- or thioglycollate-treated mice and cells purified by cell sorting were cultured in complete RPMI-1640 at $1 \times 10^{6}$ cells $/ \mathrm{mL}$ in 96 well plates and treated with either PBS, $200 \mu \mathrm{g} / \mathrm{mL} \mathrm{MSU}$, or $100 \mathrm{ng} / \mathrm{mL}$ LPS overnight at $37^{\circ} \mathrm{C}$. Supernatants were then harvested after 16 hours for cytokine analysis. 


\subsection{Whole peritoneal tissue and peritoneal leukocyte culture}

Naïve mice were euthanased by $\mathrm{CO}_{2}$ and the skin removed from the abdomen under sterile conditions. The naïve peritoneal leukocytes were collected from the mouse by a gentle lavage of the cavity with $3 \mathrm{~mL}$ PBS containing $25 \mathrm{U} / \mathrm{mL}$ heparin. Next, the ventral tissue covering the peritoneal cavity was excised and placed into cold RPMI-1640. Naïve peritoneal leukocytes were then washed twice in PBS and then re-suspended in complete RPMI-1640 at a concentration of $1 \times 10^{6}$ cells $/ \mathrm{mL}$ while the peritoneal tissue was placed into complete RPMI-1640 that had been pre-warmed at $37^{\circ} \mathrm{C}$. MSU $(200 \mu \mathrm{g} / \mathrm{mL})$ or PBS (control) were added to either peritoneal leukocytes, tissue cells or a combination of both in a 96 well plate containing $200 \mu \mathrm{L}$ of complete RPMI-1640. In experiments involving the peritoneal tissue, the visceral side of the tissue was placed face down over the top of the wells and then the lid of the 96-well plate was placed over the top so as to form a seal between the tissue and the well. The plate was then inverted to allow the media, cells and MSU to contact the peritoneal tissue. In treatments involving only leukocytes, cells were prepared in a separate 96-well plate and were not inverted. Cells were then incubated at $37^{\circ} \mathrm{C}, 5 \% \mathrm{CO}_{2}$ for 4 hours following which the supernatants were collected and assayed for cytokine levels by ELISA and cytokine bead array.

\subsection{Cytokine production from macrophages following uptake of neutrophils}

Peritoneal exudate cells from mice treated with MSU in vivo for 8 and 18 hours were harvested by lavage as described (Section 2.16.3), suspended to 1 x $10^{6}$ cells $/ \mathrm{mL}$ in complete RPMI-1640 and then split into three equal aliquots. The first aliquot of cells was used for culturing immediately $(0 \mathrm{~h})$, while the second and third groups were placed in vented $50 \mathrm{~mL}$ Falcon tubes and then put on a shaker (160 rpm, 15mm radius) in an incubator at $37^{\circ} \mathrm{C}$ for 4 and 22 hours respectively to allow for the phagocytosis of apoptotic neutrophils; after which the incubated cells were cultured in the same way as the first group. When culturing the groups of cells, cells were washed in complete RPMI-1640, the cell concentration adjusted to $1 \times 10^{6}$ cells $/ \mathrm{mL}$, and plated into 96 wells after which they were stimulated with $200 \mu \mathrm{g} / \mathrm{mL}$ MSU, $100 \mathrm{ng} / \mathrm{mL}$ LPS or $10 \mu \mathrm{L}$ PBS (control). Supernatants were collected at 8 and 24 hours after in vitro stimulation. 
Cytospin samples of cell cultures prior to in vitro stimulation were prepared and treated with Diff-Quik and peroxidase stain as described above.

\subsection{Inter-experimental variability}

Experiments measuring the generation of superoxide via WST-1 reduction had optical density readings that varied up to as much as $20 \%$ between different neutrophil donors but nevertheless maintained the same patterns of superoxide activity. Measurements involving the stimulation by MSU conditioned media for an improvement in neutrophil viability (Figure 3.9) and of IL-8 production in neutrophils (Figure 3.10) varies up to $30 \%$ between different neutrophil donors but also maintains the same pattern of differences between experiments.

\subsection{Image analyses and editing}

Microscope images of cells and tissues were acquired and optimized using AnalySIS Life Imaging Software. AnalySIS software was also used to determine width and area of cells on cytospin slides. Photoshop software was used on microscopic images to remove debris from the acquired images.

\subsection{Statistical analysis}

The Student's $t$-test was used to determine statistical significance when there were less than three treatment groups within an experiment being compared against each other. In experiments for which there were three or more groups, a one-way ANOVA and Tukey multiple comparison test was used. Statistical significance was determined when $P<$ 0.05 . 
Chapter 3

Neutrophil activation in MSU-induced inflammation 


\section{Chapter 3 \\ Neutrophil activation in MSU-induced inflammation}

\subsection{Introduction}

Neutrophils were identified as important cells in gout almost 40 years ago when Phelps and McCarty performed a key experiment demonstrating the association of neutrophils with gout using the canine joint. Dogs treated with an intrasynovial injection of MSU crystals experienced a rich leukocyte infiltrate comprised of around 80\% neutrophils (Phelps and McCarty, 1966). In this model, the degree of joint swelling was shown to be directly proportional to the amount of neutrophils infiltrating into the joint space. This was most clearly demonstrated when circulating neutrophils were selectively depleted in the blood by an intravenous treatment with the drug vinblastine: dogs that had been completely depleted of blood neutrophils displayed a complete abolishment of an acute gout inflammatory response.

Since finding that neutrophils are important in gout, researchers have undertaken to unravel the contribution of neutrophils to gout inflammation. A number of changes in neutrophil activity have been noted in in vitro conditions modelling gout inflammation. Under homeostatic conditions, neutrophils have a short lifespan undergoing apoptosis after approximately ten hours (Athens et al., 1961, Dancey et al., 1976). However, upon exposure to MSU, neutrophils delay their apoptotic program and extend their lifespan by several hours (Akahoshi et al., 1997, Tudan et al., 2000). In addition to a delay in apoptosis, direct contact with MSU crystals results in the secretion of a number of inflammatory molecules including IL-8 (Hachicha et al., 1995), $\mathrm{PGE}_{2}$ (Gilbert et al., 2003) and the release of lysosomal enzymes (Weissmann and Rita, 1972).

One of the major inflammatory products resulting from the contact of MSU with neutrophils is superoxide (Abramson et al., 1982, Simchowitz et al., 1982). Superoxide is a reactive oxygen species (ROS) that is the basis for the production of 
a host of ROS generated by neutrophils to fight infection (Section 1.10.7.4)(Babior, 2000). Since MSU crystals are a non-infectious, non-living solid material, the production of ROS is without notable benefit. The result of ROS production under these circumstances is that errant ROS production can inflict damage upon host tissue (Sies, 1997).

The enzyme responsible for the ROS production as part of the respiratory burst is the phagocytic NADPH oxidase (Phox), a functional enzyme complex that is dependent on the co-localisation of both membrane and cytosolic subunits (Nauseef et al., 1991). The membrane element, flavocytochrome $b_{558}$, is composed of a stabilising complex of $\mathrm{p} 22^{\text {phox }}$ with the flavin-centred gp91 ${ }^{\text {phox }}$. In the cytosol, there are four cytosolic components: $\mathrm{p} 40^{\text {phox }}, \mathrm{p} 47^{\text {phox }}, \mathrm{p} 67^{\text {phox }}$, which exist in a heterotrimeric complex; and the small GTPase, rac. In resting neutrophils, Phox is inactive and in an unassembled state (Fig. 3.1A). Once neutrophils become activated, there are at least three signalling pathways that result in the assembly of all of the subunits at the membrane to create the enzyme complex (Fig. 3.1B). Phosphorylation of $\mathrm{p} 47^{\text {phox }}$ by protein kinases such as protein kinase $\mathrm{C}$ and AKT exposes its $\mathrm{p} 22^{\text {phox }}$-binding domain, localising the complex to bind to flavocytochrome $b_{558}$. The production of lipid mediators by phosphatidylinositol 3-kinase (PI3K) and phospholipase D provide lipids that help bind $\mathrm{p} 40^{\text {phox }}$ and $\mathrm{p} 47^{\text {phox }}$ to the membrane. Rac dissociates from the inhibitory protein RhoGDI following guanine nucleotide exchange in the cytosol and moves to the membrane, binding to $\mathrm{p} 67^{\text {phox }}$. Assembly of all the enzyme subunits at the membrane initiates the two-electron transfer from cytosolic NADPH to the bound flavin adenine dinucleotide (FAD) (Fig. 3.1C). Two haem molecules at the centre of gp91 ${ }^{\text {phox }}$ act as electron carriers through the reduction and reoxidation of iron at the haem centres. In the final step, electrons are transferred from haem to phagosomal or extracellular oxygen in sequential one-electron transfers (Lambeth, 2004). Generation of superoxide can be observed within 30 seconds of neutrophil stimulation, demonstrating the speed at which these processes can occur and the readiness of neutrophils to respond to inflammatory stimuli. 
Figure 3.1. The NADPH oxidase enzyme. (A) The inactive enzyme complex consists of a membrane component (gp91 ${ }^{\text {phox }}$ and $\mathrm{p} 22^{\text {phox }}$ ) and a number of cytosolic components ( $\mathrm{p} 40^{\text {phox }}, \mathrm{p} 47^{\text {hox }}, \mathrm{p} 67^{\mathrm{phox}}$ and the small GTPase rac). (B) Three events occur once neutrophils are stimulated: p47phox is phosphorylated by protein kinases such as protein kinase $\mathrm{C}$ and AKT, causing p47phox to bind to p22phox at the membrane; Phosphatidylinositol 3-kinase and phospholipase D produce phosphorylated phosphatidylinositols (PtdInsP) and phosphatidic acid facilitating binding of p40phox and p47phox to the membrane complex; and rac is released from the inhibitory protein RhoGDI by an enzyme mediated exchange of GDP for GTP on the rac protein, following which, rac binds to the enzyme complex at the membrane. (C) Assembly of the cytosolic and membrane components form the active enzyme complex. The enzyme catalyses the two electron transfer from NADPH to FAD and through the heme centres by a process catalysed by iron reduction and re-oxidation. Single electrons are then transferred to oxygen molecules resulting in the generation of two superoxide molecules per NADPH into the phagosome or extracellular space. Figure adapted from (Lambeth, 2004). 

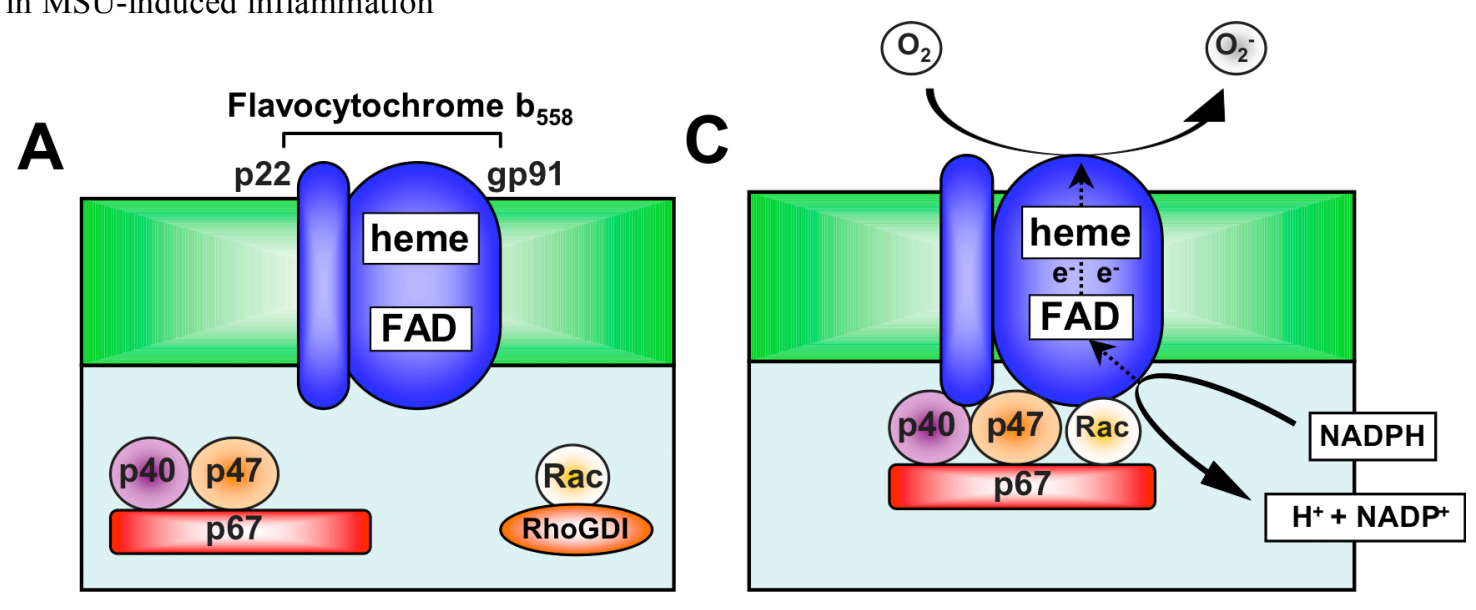

B

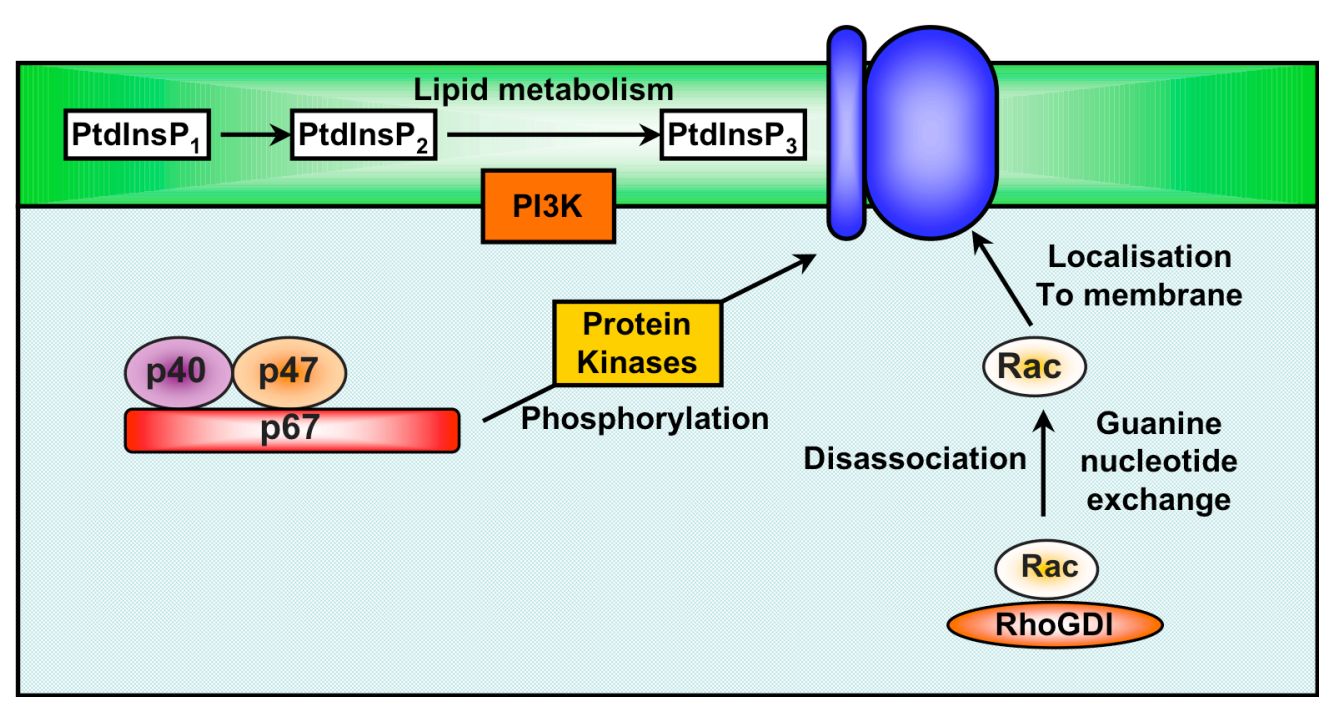


The process by which MSU crystals activate NADPH oxidase is currently unknown. One potential pathway of activation by MSU is through the phosphorylation of PKC and the activation of phospholipase D that leads to the activation of NADPH oxidase (Fig. 3.1B) (Naccache et al., 1993). However, PKC inhibitors cause only a moderate reduction in MSU-induced superoxide activity (Naccache et al., 1991) and as such, a number of pathways are likely to be involved.

The current model of neutrophil recruitment and activation in gout is outlined in Figure 3.2. Once MSU crystals have formed in the joint, local innate immune cells interact with the crystals and produce cytokines and chemokines that recruit neutrophils into the joint space (Section 1.10.7). When neutrophils infiltrate into the joint they are exposed to MSU crystals, resulting in the activation and subsequent production of neutrophil IL-8, superoxide and a delay in apoptosis. Together, these activities augment the accumulation and activation of neutrophils and induce the production of ROS that inflict inflammatory symptoms and physical damage in the local joint environment.

The studies leading up to this model have primarily focussed on a single aspect of neutrophil activation, that is; either viability (Akahoshi et al., 1997), IL-8 production (Hachicha et al., 1995), or superoxide production alone (Simchowitz et al., 1982), emphasizing the engagement of those activities via direct contact with MSU. However, neutrophils are likely to be exposed to inflammatory mediators prior to contact with MSU that may influence neutrophil responses. This idea has been partially explored in a study showing that extraneous $\mathrm{TNF} \alpha$ can prime neutrophil superoxide production in response to MSU (Burt and Jackson, 1997). However, these authors did not investigate the effect of MSU-generated cytokines on neutrophil viability or neutrophil cytokine production. This chapter explores how human neutrophil responses to MSU-induced inflammation are affected by inflammatory factors that are present in the environment prior to direct contact with MSU. 


\section{Blood vessel}

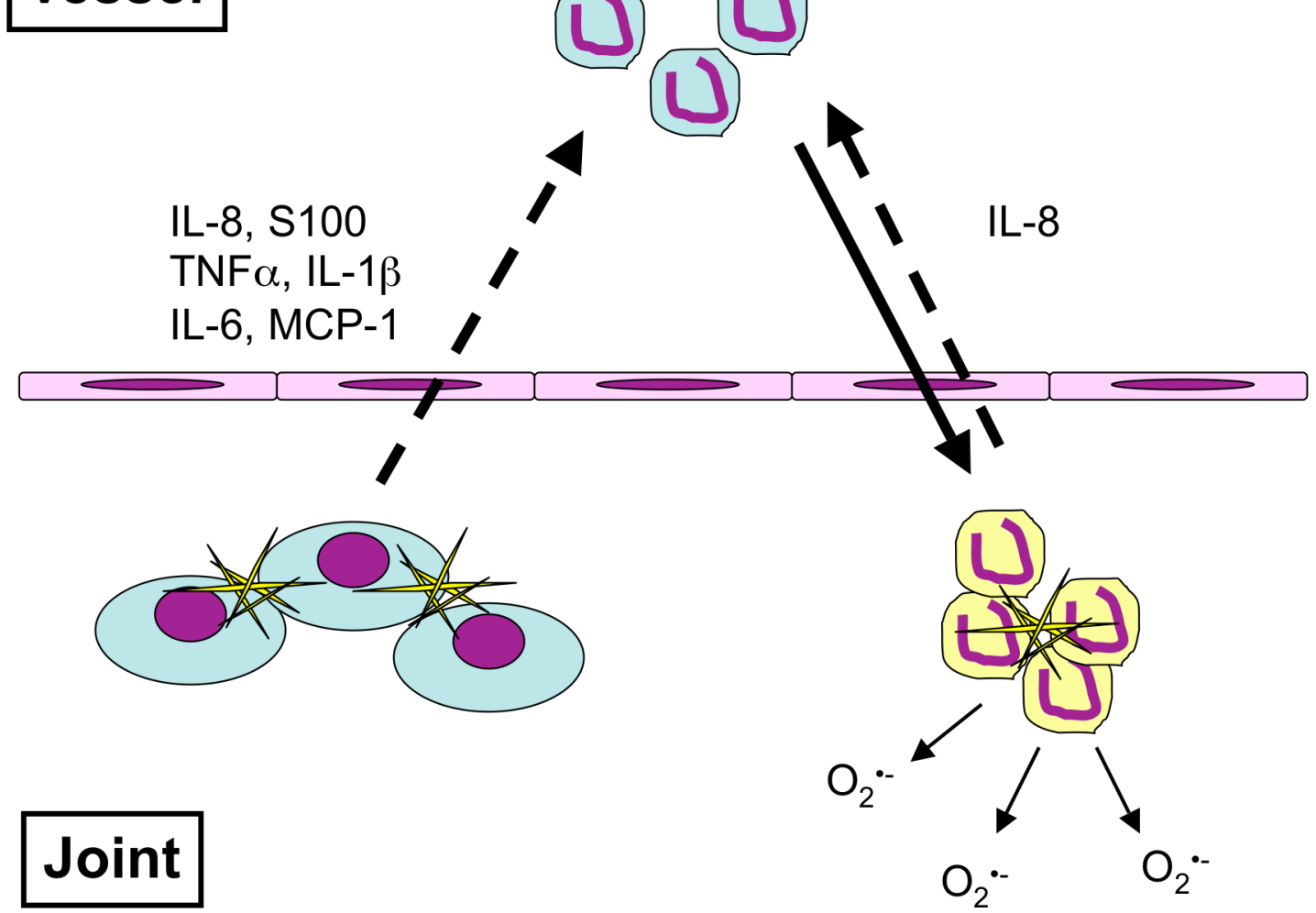

Figure 3.2. Current model of neutrophil activation in gout. MSU crystals stimulate local cells in the joint to produce a number of pro-inflammatory cytokines that recruit neutrophils into the joint space. Neutrophils then make contact with MSU and are activated (yellow) to produce IL-8 and superoxide. Contact with MSU also results in an improvement in neutrophil viability. 


\subsection{Results}

\subsubsection{Measuring neutrophil superoxide production}

Activation of neutrophils is associated with the production of superoxide. Previous research has shown that MSU crystals stimulate neutrophils to produce superoxide. To confirm this, human neutrophils were cultured in HBSS and exposed to MSU. Superoxide production was measured using the dye, WST-1. Consistent with previous research, neutrophils produced superoxide in response to MSU (Fig. 3.3). Superoxide was produced in a dose-dependent manner that reached a maximal response at $0.5 \mathrm{mg} / \mathrm{mL}$.

Next, various culture conditions were tested to optimise the conditions for superoxide production. PBS and RPMI-1640 were explored as alternative assay media, with superoxide production compared against neutrophils activated in HBSS. In addition, different serum supplementations were tested, including different serum concentrations and serum from different species.

First, the effect of different media conditions on the background level of superoxide production by unstimulated human neutrophils was compared: In the absence of serum, background production of superoxide by neutrophils in RPMI-1640 was twice that of neutrophils in PBS and HBSS (Fig. 3.4). The addition of autologous human serum below 10\% caused an unexpected increase in background superoxide production. This effect was more pronounced in more complex media, to the extent that neutrophils in RPMI-1640/serum produced a greater amount of superoxide than in HBSS/serum, which produced more than in PBS/serum (See Appendix for formulations of media). A substantial increase in background superoxide production peaked at $0.22 \%$ in all media, indicating that very low concentrations of serum stimulated superoxide production. At 10\% serum, background levels were closer to those observed in neutrophils cultured in the absence of serum. The stimulatory effect of low-level serum appeared to be species specific as the same activity was not observed in the presence of FBS. In fact, even the smallest amount of FBS $(0.11 \%)$ completely inhibited the background production of superoxide. This revealed that the stimulatory effect of low human serum on human neutrophil superoxide production is not a universal feature of any type of serum. 


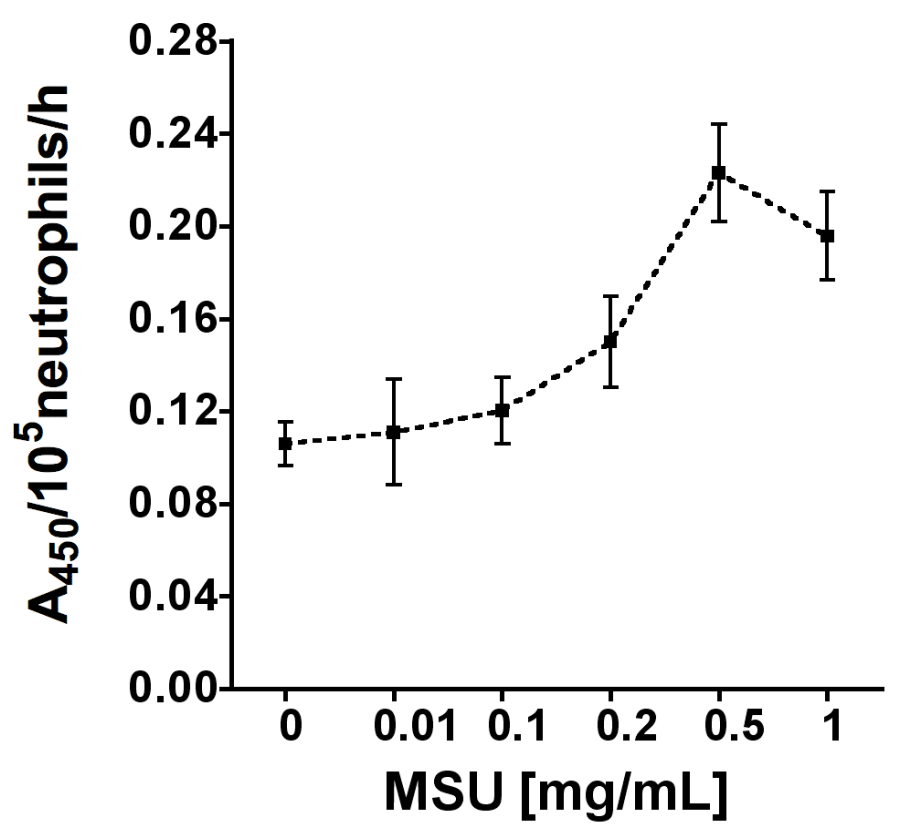

Figure 3.3. MSU stimulated production of superoxide by human neutrophils. Neutrophils were isolated from peripheral blood and suspended in serum-free HBSS $\left(1.5 \times 10^{5}\right.$ cells, $\left.150 \mu \mathrm{L}\right)$. Neutrophils were stimulated with $200 \mu \mathrm{g} / \mathrm{mL}$ MSU in the presence of the dye WST-1 $(250 \mu \mathrm{g} / \mathrm{mL})$. The production of superoxide was measured in $100 \mu \mathrm{L}$ of harvested cell- and MSU-free supernatant by WST-1 reduction at absorbance $450 \mathrm{~nm}\left(\mathrm{~A}_{450}\right)$ after $1 \mathrm{~h}$. Measurements were performed in triplicate. Values represent mean \pm S.E.M. Results are representative of three separate experiments. 


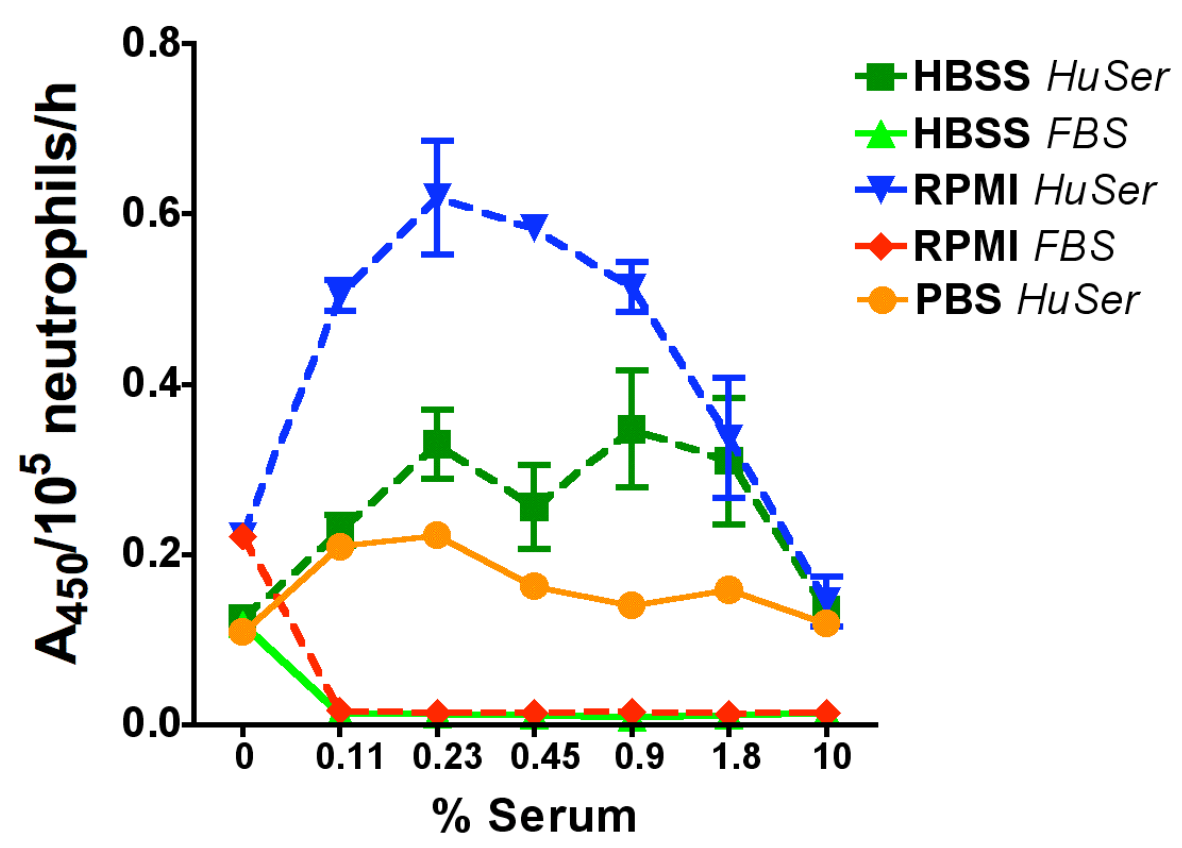

Figure 3.4. The effect of different media conditions on background superoxide production by human neutrophils. Neutrophils were isolated from peripheral blood and cultured in a number of different media conditions $\left(1.5 \times 10^{5}\right.$ cells, $150 \mu \mathrm{L})$ in the absence of stimuli. Culture conditions included combinations of PBS, HBSS or RPMI-1640 (RPMI) with autologous human serum (HuSer) and fetal bovine serum $(F B S)$. The production of superoxide was measured by WST-1 reduction $(250 \mu \mathrm{g} / \mathrm{mL})$ at absorbance $450 \mathrm{~nm}\left(\mathrm{~A}_{450}\right)$ in $100 \mu \mathrm{L}$ of the cell- and MSUfree supernatant after $1 \mathrm{~h}$. Measurements were performed in triplicate. Values represent mean \pm S.E.M. $\left(\mathrm{V}_{\max }\right)$. Results are representative of three separate experiments. 
In summary, the results above suggested that the complexity of the medium and the concentration and source of serum were factors that affected superoxide production by neutrophils. In an in vivo setting, neutrophils are likely to have a number of nutrients present in the surrounding milieu (modelled by RPMI-1640), and not just a few salts (modelled by PBS and HBSS). Therefore, despite an elevated level of background superoxide production, RPMI-1640 was chosen as the most appropriate culture medium to measure superoxide activity.

\subsubsection{Superoxide production by MSU-stimulated neutrophils is affected by human serum concentration.}

As serum concentration had an obvious effect on otherwise unstimulated neutrophils, this raised the question of how human serum concentration alters the superoxide response by neutrophils stimulated with MSU crystals.

It is already known that serum proteins can affect MSU-induced superoxide production. Most of this previous research is based on the idea that MSU becomes coated by serum proteins both in vivo and in vitro, and these proteins (including apolipoprotein B) inhibit neutrophil responses to MSU (Terkeltaub et al., 1984, Ortiz-Bravo et al., 1993). It has also been shown that MSU crystals can activate complement proteins in plasma, leading to an enhanced superoxide response (Boogaerts et al., 1983). However, it has never been considered that serum alone may play a role in neutrophil activation in gout. The effect of low serum levels has so far remained undiscovered because MSU stimulation of human neutrophils has typically been performed either in the absence of serum (Burt and Jackson, 1997, Barabe et al., 1998) or at 10\% human serum (Abramson et al., 1982). Therefore, the level of human serum present in the culture medium may be an important aspect in modelling conditions that correctly reflect the in vivo situation.

In the circulation, neutrophils are exposed to very high levels of serum and to low level serum in a swollen joint in inflammatory conditions (Schmid and Macnair, 1956, Kushner and Somerville, 1971). To incorporate serum effects into MSU stimulated superoxide production, purified human neutrophils were cultured in the presence of different concentrations of autologous serum and stimulated with MSU. 
A concentration of $500 \mu \mathrm{g} / \mathrm{mL}$ is commonly used to induce a maximal superoxide response (Fig. 3.3); however, to avoid missing possible potentiation as well as suppression of superoxide by serum, responses were tested at a suboptimal MSU concentration of $200 \mu \mathrm{g} / \mathrm{mL}$. The addition of PBS to the culture medium instead of MSU was used to determine the effect of serum alone.

As shown in Figure 3.5, unstimulated neutrophils exhibited the same background response to serum concentration that was observed in the previous experiment (Fig. 3.4). A low level of human serum caused an increase in the base level production of superoxide, and as serum concentration was increased, the base level superoxide production decreased, re-affirming that serum itself directly affects superoxide production. Interestingly, when neutrophils were stimulated with MSU they produced significantly lower levels of superoxide compared with serum alone up to a concentration of $2.5 \%$. At serum concentrations above $2.5 \%$ serum MSU stimulation increased the amount of superoxide produced by neutrophils compared to serum alone returning to background levels at $10 \%$ serum concentration.

To determine whether the serum effect was due to proteins present in the serum, this experiment was repeated using human serum that had been heat inactivated at $56^{\circ} \mathrm{C}$ for 30 minutes. Heat inactivation of serum abrogated the increase in base level superoxide observed at low serum concentrations, indicating the presence of stimulating proteins in the serum that were sensitive to heat treatment (Fig. 3.5). Neutrophils cultured in heat inactivated serum displayed a similar pattern of responsiveness to MSU as neutrophils cultured in normal serum; that is: in low serum concentrations, MSU reduced background superoxide production, while in higher serum concentrations MSU increased superoxide production (Fig. 3.5). To determine if the complex effects of serum and MSU on neutrophil superoxide production were dependent on autologous serum, neutrophils from a single donor were exposed to serums from four different individuals and then stimulated with MSU. Neutrophils showed the same pattern and degree of both background and MSU-stimulated superoxide production as neutrophils cultured in autologous serum 


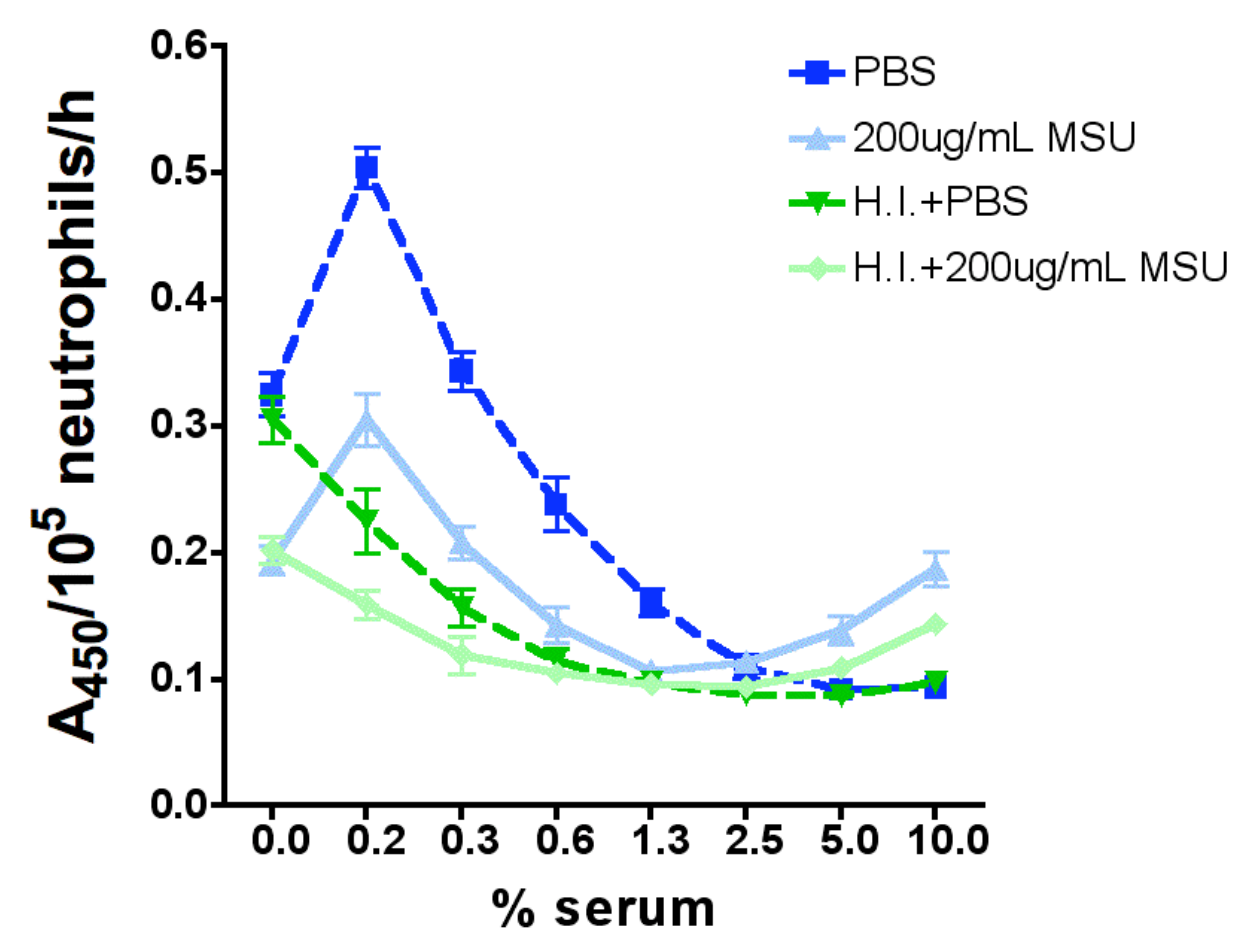

Figure 3.5. The effect of serum on the production of superoxide by human neutrophils. Neutrophils were isolated from peripheral blood and cultured $\left(1.5 \times 10^{5}\right.$ cells, $150 \mu \mathrm{L}$ RPMI) in normal autologous serum or heat inactivated autologous serum (H.I.) and were unstimulated (10 $\mu \mathrm{L}$ PBS) or stimulated with $200 \mu \mathrm{g} / \mathrm{mL} \mathrm{MSU}$ crystals. The production of superoxide was measured by WST-1 reduction $(250 \mu \mathrm{g} / \mathrm{mL})$ in $100 \mu \mathrm{L}$ of cell- and MSU-free supernatant after $1 \mathrm{~h}$ at absorbance $450 \mathrm{~nm}\left(\mathrm{~A}_{450}\right)$. Values represent mean \pm S.E.M. where $\mathrm{n}=10$ readings per datapoint. Results are representative of three separate experiments. 


\section{Serum 1}

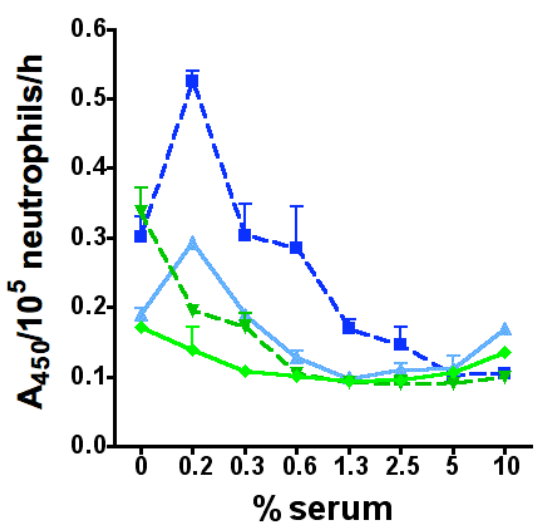

Serum 3

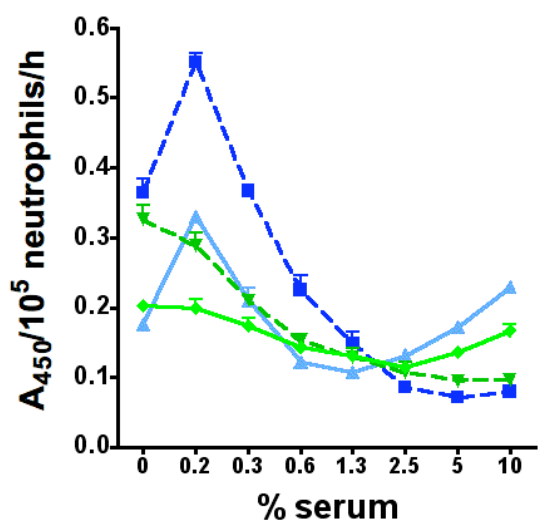

Serum 2

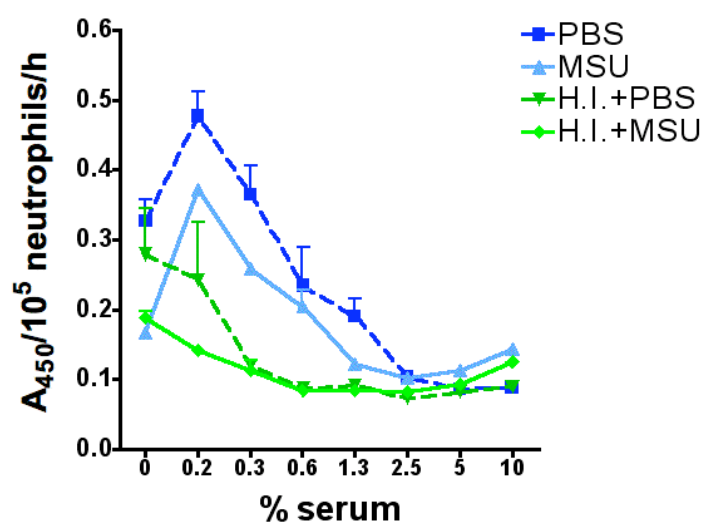

Serum 4

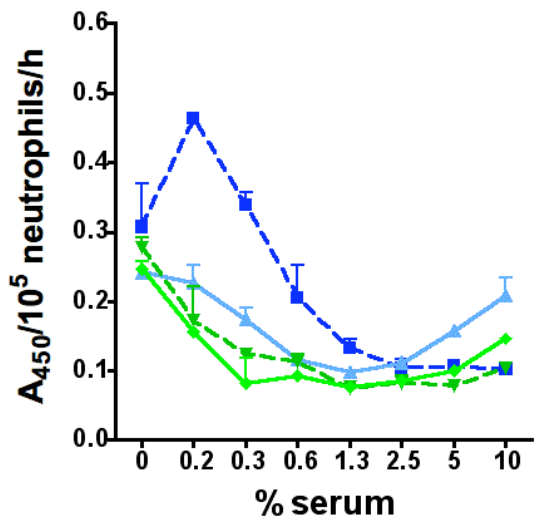

Figure 3.6. The effect of non-autologous serum on the production of superoxide by human neutrophils. Neutrophils were isolated from peripheral blood and cultured $\left(1.5 \times 10^{5}\right.$ cells, $150 \mu \mathrm{L}$ RPMI) in normal and heat inactivated (H.I) serum from four separate donors. Serum 1 represents the results from neutrophils in the presence of autologous serum, while Serums 2-4 represent responses from neutrophils incubated with serums from heterologous donors. Neutrophils were unstimulated (10 $\mu \mathrm{L}$ PBS) or stimulated with $200 \mu \mathrm{g} / \mathrm{mL}$ MSU crystals. The production of superoxide was measured by WST-1 reduction $(250 \mu \mathrm{g} / \mathrm{mL})$ in $100 \mu \mathrm{L}$ of cell- and MSU-free supernatant after $1 \mathrm{~h}$ at absorbance $450 \mathrm{~nm}\left(\mathrm{~A}_{450}\right)$. Measurements were performed in triplicate. Values represent mean + S.E.M. 
(Fig. 3.6). This indicated that the effect of human serum on human neutrophil production of superoxide was not dependent on the autologous donor serum.

The data presented in the previous section indicate that stimulation of neutrophils by MSU in vitro can cause conflicting results depending on the chosen culture conditions, ranging from inhibitory (low serum conditions) to stimulatory (high serum conditions). The implications of this will be discussed later (Section 3.3). Since neutrophils are exposed to different concentrations of serum in vivo depending on their location (peripheral blood, joints, etc.), the most appropriate conclusion from these results is that although neutrophils are clearly stimulated by MSU to produce superoxide, the magnitude of the response is influenced by presence of serum and other proteins.

\subsubsection{The effect of MSU on neutrophil viability and IL-8 production}

Neutrophil superoxide production is only one measurement of neutrophil activation by MSU. Neutrophil activation can also be observed as a delay in neutrophil apoptosis - as measured by an improvement in neutrophil viability - in combination with an increase in the production of pro-inflammatory cytokines, such as IL-8. Hence, the effect of MSU on neutrophil survival and cytokine production was determined. To do this, purified human neutrophils were cultured with or without MSU over 24 hours, and the effect on neutrophil viability and cytokine production quantified. As soluble cytokines were being measured, cells were cultured in $10 \%$ FBS rather than human serum, eliminating the likely complication of high background levels of human cytokines present in human donor serum.

Neutrophil viability was measured by flow cytometry using propidium iodide (PI), and fluorescently labelled Annexin- $\mathrm{V}$. The combination of these stains clearly delineated viable from apoptotic cells (Fig. 3.7A). Viable cells were Annexin- $\mathrm{V}^{-} / \mathrm{PI}^{-}$; whereas apoptotic cells were Annexin- $\mathrm{V}^{+} / \mathrm{PI}^{-}$; necrotic cells were Annexin- $\mathrm{V}^{-} / \mathrm{PI}^{+}$; and late stage dead cells from both apoptosis and necrosis were Annexin- $\mathrm{V}^{+} / \mathrm{PI}^{+}$. 
Figure 3.7. The effect of MSU on neutrophil viability and cytokine production. Human neutrophils $\left(2 \times 10^{5}\right.$ cells; $\left.200 \mu \mathrm{L}\right)$ purified from peripheral blood were cultured with or without $200 \mu \mathrm{g} / \mathrm{mL}$ MSU. The effect of MSU on neutrophil viability was measured by flow cytometry. A. Gating strategy for determining neutrophils that are viable (double negative), apoptotic (Annexin- $\mathrm{V}^{+}, \mathrm{PI}^{-}$) and dead $\left(\mathrm{PI}^{+}\right)$. B. $\mathrm{Viability}$ profile of unstimulated neutrophils cultured over 24h. C. Viability profile of MSU stimulated neutrophils over 24h. D. Cytokines produced by unstimulated neutrophils. E. Cytokines produced by MSU stimulated neutrophils. Cytokine production from neutrophils was measured by cytokine bead array. Measurements were performed in triplicate. Values represent mean \pm S.E.M. Results are representative of three separate experiments. 
A

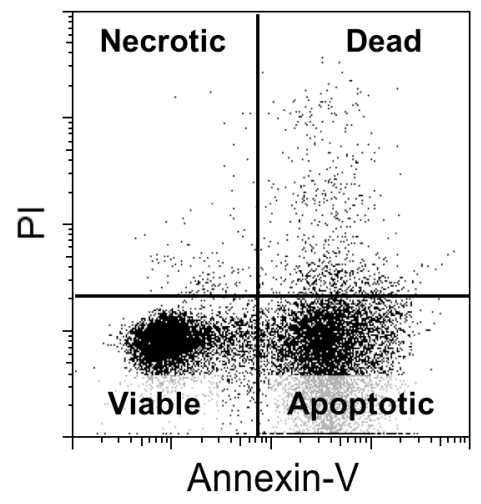

B

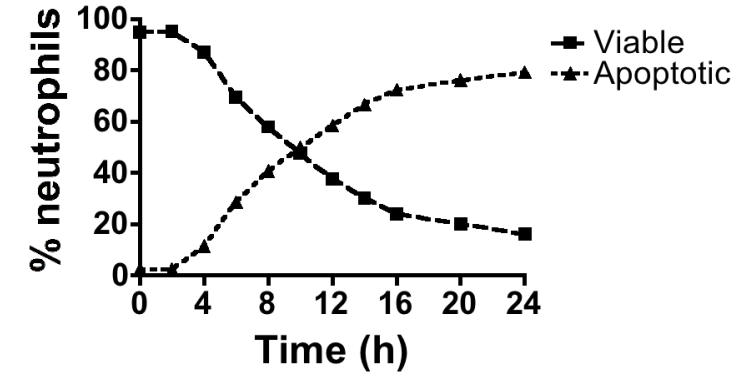

D

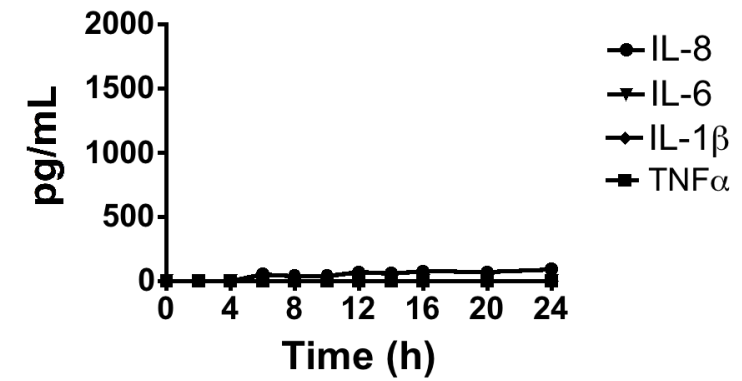

c
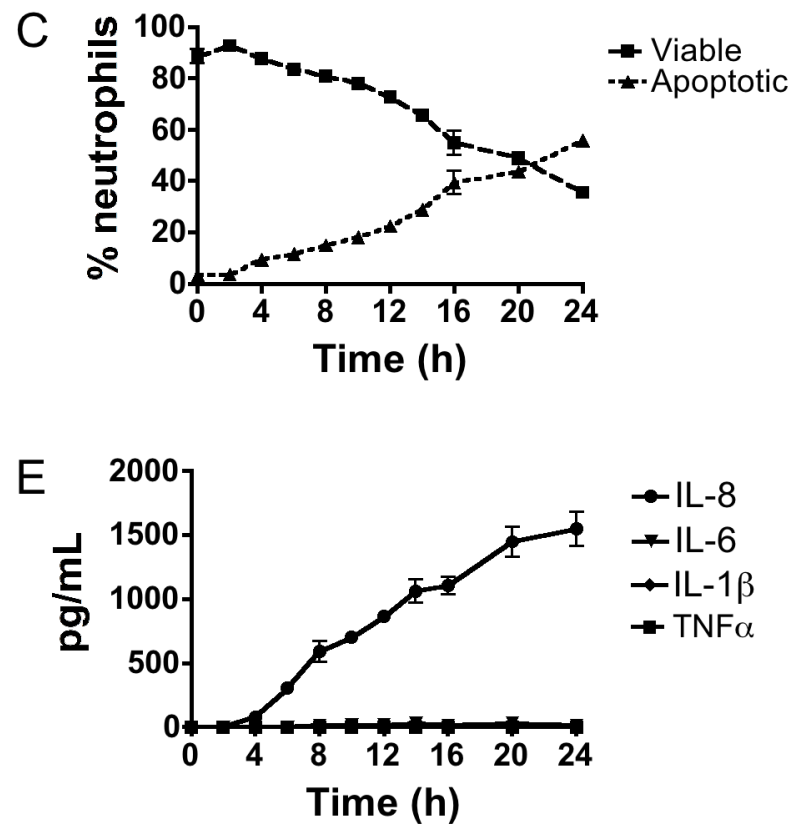
In unstimulated neutrophil cultures the number of apoptotic neutrophils began to increase after 2 hours; and after 10 hours, 50\% of neutrophils were apoptotic (Fig. 3.7B). In neutrophils that were cultured with MSU, neutrophil apoptosis was delayed such that $50 \%$ of cells were still viable at 20 hours (Fig. 3.7C). This indicated that exposure to MSU approximately doubled the half-life of neutrophils in culture. In addition, after 24 hours survival was twice as high in MSU-stimulated neutrophils than in unstimulated neutrophils (Fig. 3.7B-C). The increase in survival time following exposure to MSU was consistent with reports from other researchers (Akahoshi et al., 1997).

To determine the effect of MSU on neutrophil cytokine production, supernatants from the neutrophil viability experiments above were assayed for the production of a number of classical inflammatory cytokines. Of the cytokines assayed (TNF $\alpha$, IL$1 \beta$, IL-6 and IL-8), only IL-8 was detectable in the culture supernatant of neutrophils (Fig. 3.7D and E). Unstimulated neutrophils produced a low background level of IL8, whereas neutrophils produced a large amount of IL-8 in response to MSU (Fig. 3.7D and $\mathrm{E})$.

Together, these results show that direct exposure to MSU crystals activates neutrophils resulting in an increase in cell survival and the production of the proinflammatory cytokine IL-8.

\subsubsection{The effect of mixed cell populations on MSU-induced neutrophil activation}

In gout, neutrophils are not the sole leukocytes present in the joint during an acute inflammatory attack; several other cell types are present including monocytes, macrophages and lymphocytes, that may contribute to the inflammatory response and affect neutrophil activation and function. To mimic the effect of other cell types on neutrophil activation, total human white blood cells (WBCs) were used to model the mixture of inflammatory cells found in the joint over the course of an inflammatory response. Human blood WBCs are composed of 50-70\% neutrophils and contain a mixture of cells including monocytes and lymphocytes. One drawback is that WBCs do not contain macrophages, and so the predominant mononuclear phagocyte in the blood is the monocyte. Nevertheless, WBCs are the closest model 
of the mixture of inflammatory cells found in the human joint without implementation of complex mixtures of immortalised cell lines.

The effect of mixed cell populations on neutrophil viability, was measured by Annexin-V and PI staining. Identification of human neutrophils was performed by flow cytometry using anti-CD15 (Fig. 3.8A). As shown in Figure 3.8B, neutrophils survived longer in the presence of unstimulated WBCs than neutrophils alone $(50 \%$ survival after $20 \mathrm{~h}$ and $10 \mathrm{~h}$ respectively) (Figs. 3.7B and 3.8B). In fact, neutrophil survival in unstimulated WBCs was similar to the survival of MSU-stimulated purified neutrophils, with both sets of neutrophils displaying $50 \%$ viability at 20 hours (Figs. 3.7C and 3.8B). When mixed WBCs were stimulated with MSU, there was a dramatic improvement in neutrophil survival. After 24 hours, 80\% of neutrophils in MSU-stimulated WBCs were still viable (Fig. 3.8C). These results indicate that the presence of other leukocytes significantly enhances neutrophil survival in both the presence and absence of MSU.

Improved neutrophil survival is often associated with the presence of inflammatory cytokines. Therefore, the production of pro-inflammatory cytokines in the WBC cultures was measured. With respect to WBCs alone, only background levels of IL8 were observed (Fig. 3.8D). However, stimulation of WBCs with MSU produced greater than three fold higher levels of IL-8 than observed in MSU-stimulated neutrophils alone, as well as high levels of additional cytokines including IL-6, IL$1 \beta$ and TNFo (Fig. 3.8E). As WBCs were a mixed cell population, cell supernatants did not allow the identification of the cellular source of these cytokines. However, I had previously shown that isolated neutrophils only produced IL-8 (Fig. 3.7E) indicating that cells other that neutrophils were likely to be the major producers of IL-6, TNF $\alpha$ and IL-1 $\beta$ and may even be additional producers of IL- 8 because the contribution of neutrophil IL-8 cannot be determined directly. Despite this limitation, these results show that neutrophil survival was greatly increased in mixed cell populations and increased even further following stimulation with MSU, and that this increase is associated with the production of high levels of cytokines. 
Figure 3.8. The effect of other leukocytes on MSU-stimulated neutrophil viability. Total white blood cells (WBCs) $\left(2 \times 10^{5}\right.$ cells; $\left.200 \mu \mathrm{L}\right)$ were purified from peripheral blood and cultured with or without $200 \mu \mathrm{L}$ MSU. The effect of MSU on neutrophil viability in the mixed cell population was determined by flow cytometry, using CD15 to identify neutrophils. A. CD15 gating of neutrophils. B. Viability profile of neutrophils in unstimulated WBCs over 24h. C. Viability profile of neutrophils in MSU-stimulated WBCs over 24h. D. Cytokines produced by unstimulated WBCs. E. Cytokines produced by MSU stimulated WBCs. Cytokine production from WBCs was measured by cytokine bead array. Measurements were performed in triplicate. Values represent mean \pm S.E.M. Results are representative of three separate experiments. 


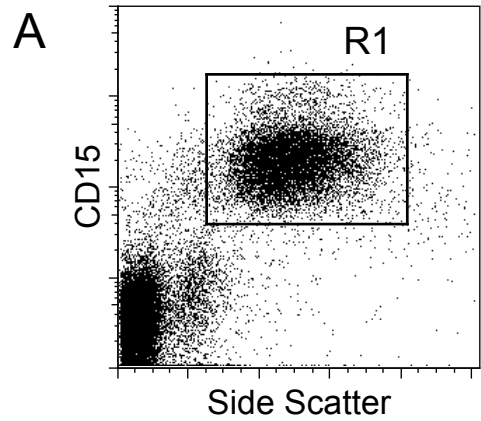

B

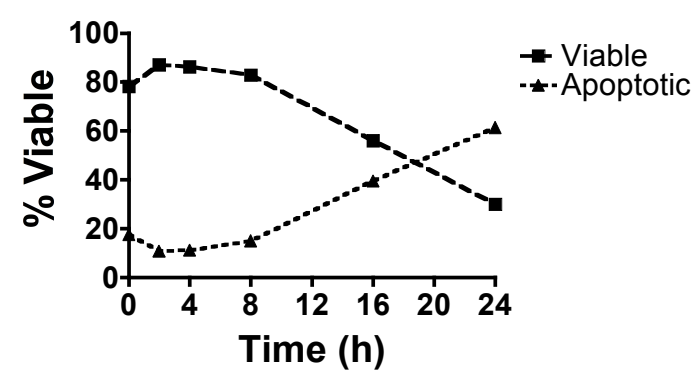

D

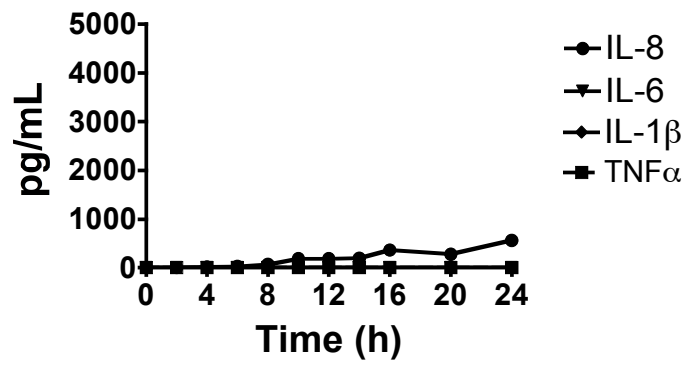

C

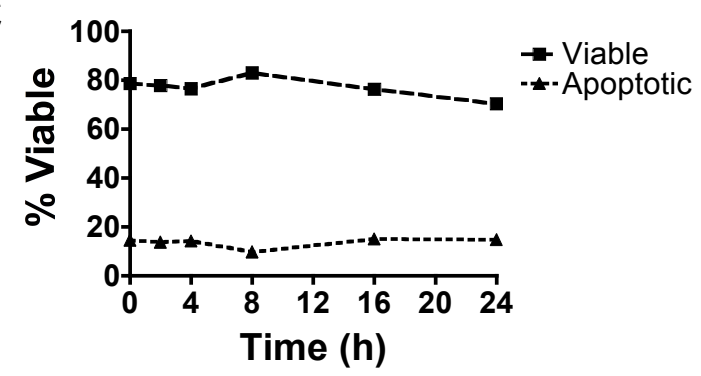

E

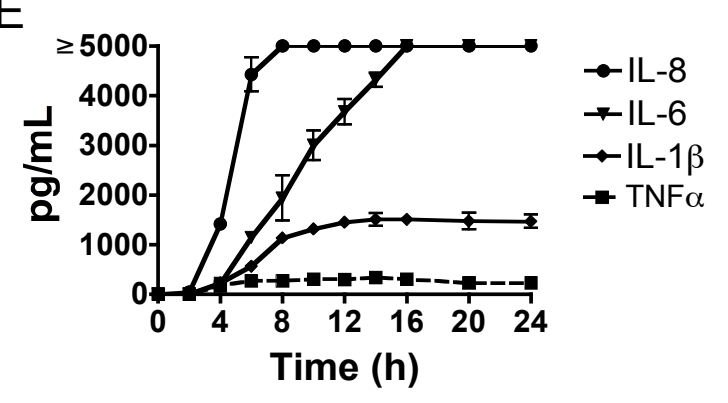




\subsubsection{The effect of soluble mediators on neutrophil activation and function}

A number of human studies have shown the potential of peripheral blood mononuclear cells (PBMCs), in particular of human monocytes, to produce proinflammatory cytokines following exposure to MSU (Guerne et al., 1989, di Giovine et al., 1991). Elevated production of cytokines by MSU-stimulated WBCs implicated PBMCs as the source of these cytokines. Enhanced neutrophil survival was associated with increased cytokine levels; however, neutrophil survival was enhanced even in the absence of MSU stimulation. Therefore, the effect of PBMCs on neutrophil survival may have either been due to the increased levels of inflammatory mediators, by cell-to-cell contact between neutrophils and PBMCs, or both.

In the process of neutrophil recruitment under MSU-stimulated conditions, circulating neutrophils are likely to encounter soluble signals released into the circulation by MSU-stimulated resident cells in the joint. This would occur prior to contact with activated cells in the joint, or with MSU. For this reason, MSU-induced soluble mediators seem likely candidates for the viability-enhancing effects of MSU on neutrophils in mixed cell populations. In addition to improved viability, MSUinduced soluble mediators may affect other aspects of neutrophil activation such as the production of IL-8 and superoxide.

To assess whether the observed increase in neutrophil survival required cell-cell contact or was orchestrated by soluble mediators, PBMCs were isolated from human blood and used to generate three types of media for experiments:

- Unconditioned media; a control media (RPMI-1640; 10\% human serum) that had not been exposed to cells nor MSU

- Conditioned media; media (RPMI-1640; 10\% human serum) that had been incubated with PBMCs in the absence of any stimulus but contained PBS as a control for MSU

- MSU-conditioned media; media (RPMI-1640; 10\% human serum) that had been derived from PBMCs that had been stimulated with $200 \mu \mathrm{g} / \mathrm{mL}$ MSU. 


\subsubsection{Neutrophil survival is improved by MSU-induced soluble mediators and} does not require cell-cell contact

To determine the effect of soluble mediators on neutrophil survival, neutrophils were exposed to the three different media described above. Approximately 50\% of human neutrophils cultured with unconditioned media were still viable after 24 hours compared to $68 \%$ of neutrophils cultured in conditioned media (Fig. 3.9, top left and top middle). This increased to $85 \%$ viability in neutrophils exposed to MSUconditioned media (Fig. 3.9, top right), indicating that the improvement in neutrophil survival observed in stimulated mixed cell populations was caused by soluble mediators, and did not require cell-to-cell contact. As in the earlier neutrophil experiment, viability of neutrophils in unconditioned media increased to $73 \%$ following MSU treatment (Fig. 3.7B and Fig. 3.9, bottom left). In contrast, neutrophils that were cultured in MSU-stimulated conditioned media showed no further improvement in viability when MSU was added to the culture (Fig. 3.9, bottom right). These results demonstrate that soluble mediator stimulation of neutrophils has an equal if not greater influence on neutrophil viability compared with direct interaction of neutrophils with MSU.

\subsubsection{Neutrophil IL-8 production is activated by MSU-induced soluble mediators}

Soluble mediators from MSU-stimulated PBMCs had such a profound effect on neutrophil viability that the effect of these mediators on neutrophil IL-8 production was next examined. Since soluble IL-8 may have been derived either from the prepared conditioned media or from newly stimulated neutrophils, intracellular cytokine staining was used to focus on neutrophil IL-8 production.

As shown in Figure 3.10, approximately 14\% of neutrophils cultured in conditioned and unconditioned media were IL- $8^{+}$compared to almost $60 \%$ IL- $8^{+}$neutrophils in MSU-conditioned media (Fig. 3.10, upper panels). This suggests that soluble mediators from MSU-stimulated cells have the capacity to activate neutrophils to produce IL-8, without neutrophils being in direct contact with MSU. When neutrophils in conditioned and unconditioned media were stimulated directly with MSU, only $26 \%$ of cells were IL- $8^{+}$compared with $59.8 \%$ of neutrophils cultured with MSU-conditioned media alone (Fig. 3.10, bottom left and middle, top right). 
Figure 3.9. The effect of soluble mediators derived from MSU-stimulation on neutrophil viability. Human neutrophils were purified from peripheral blood and cultured for $24 \mathrm{~h}\left(5 \times 10^{5}\right.$ cells, $\left.500 \mu \mathrm{L}\right)$ in the presence of untreated media (Unconditioned, left hand panels), media conditioned by unstimulated mononuclear cells (Conditioned, middle panels), or media conditioned by MSU-stimulated mononuclear cells (MSU-conditioned, right hand panels). Neutrophil cultures were also cultured in the presence of $200 \mu \mathrm{g} / \mathrm{mL}$ MSU (lower panels). Neutrophil viability was determined by Annexin-V/PI staining. Results are representative of three separate experiments. 

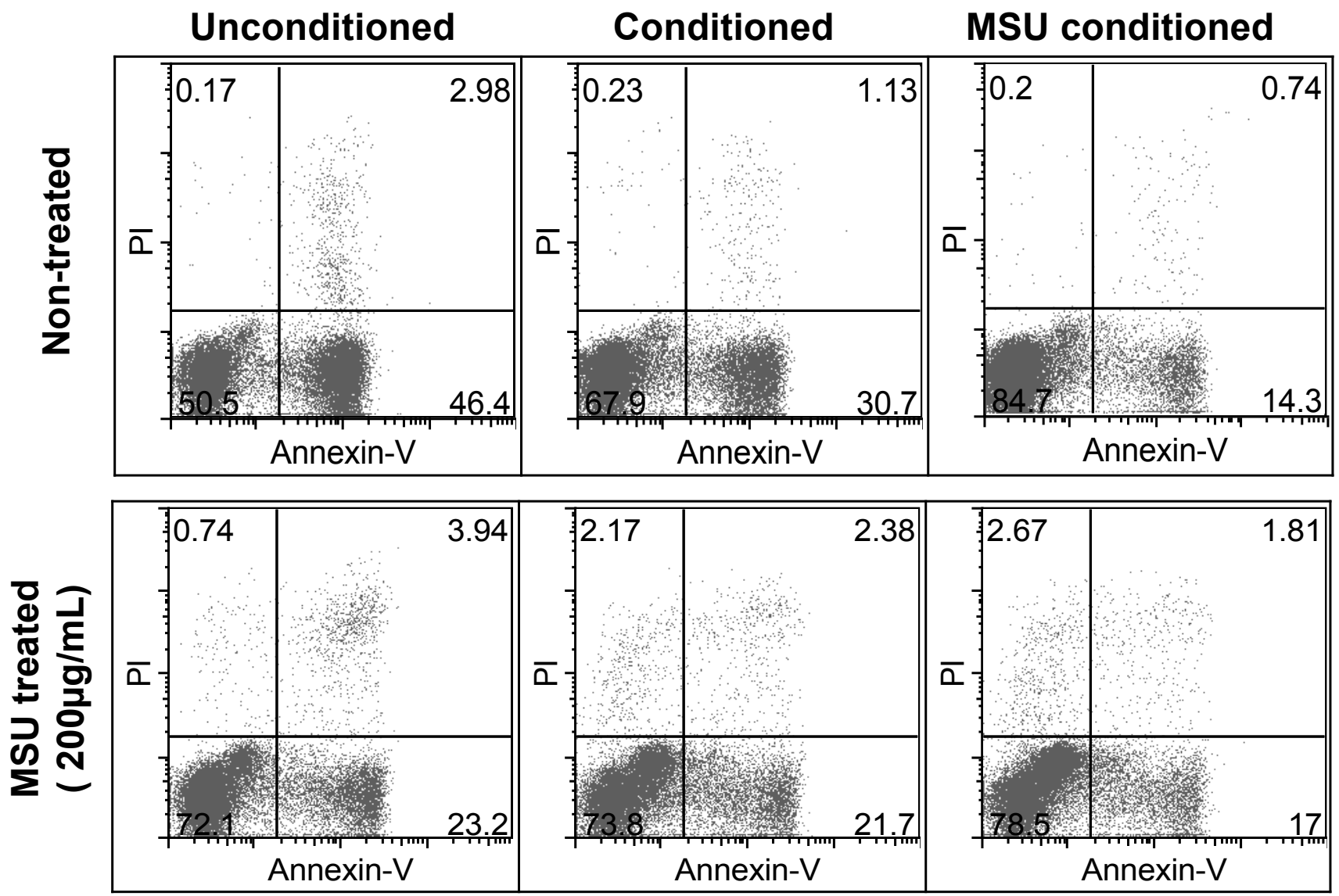
Figure 3.10. The effect of soluble mediators derived from MSU-stimulation on neutrophil IL-8 production. Human neutrophils were purified from peripheral blood and cultured $\left(5 \times 10^{5}\right.$ cells, $\left.500 \mu \mathrm{L}\right)$ for $4 \mathrm{~h}$ in the presence of untreated media (Unconditioned, second column panels), media conditioned by unstimulated mononuclear cells (Conditioned, third column panels), or media conditioned by MSU-stimulated

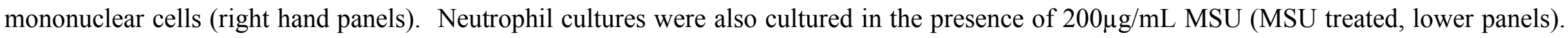
Neutrophil IL-8 production was then determined by intracellular staining. Results are representative of three separate experiments. 

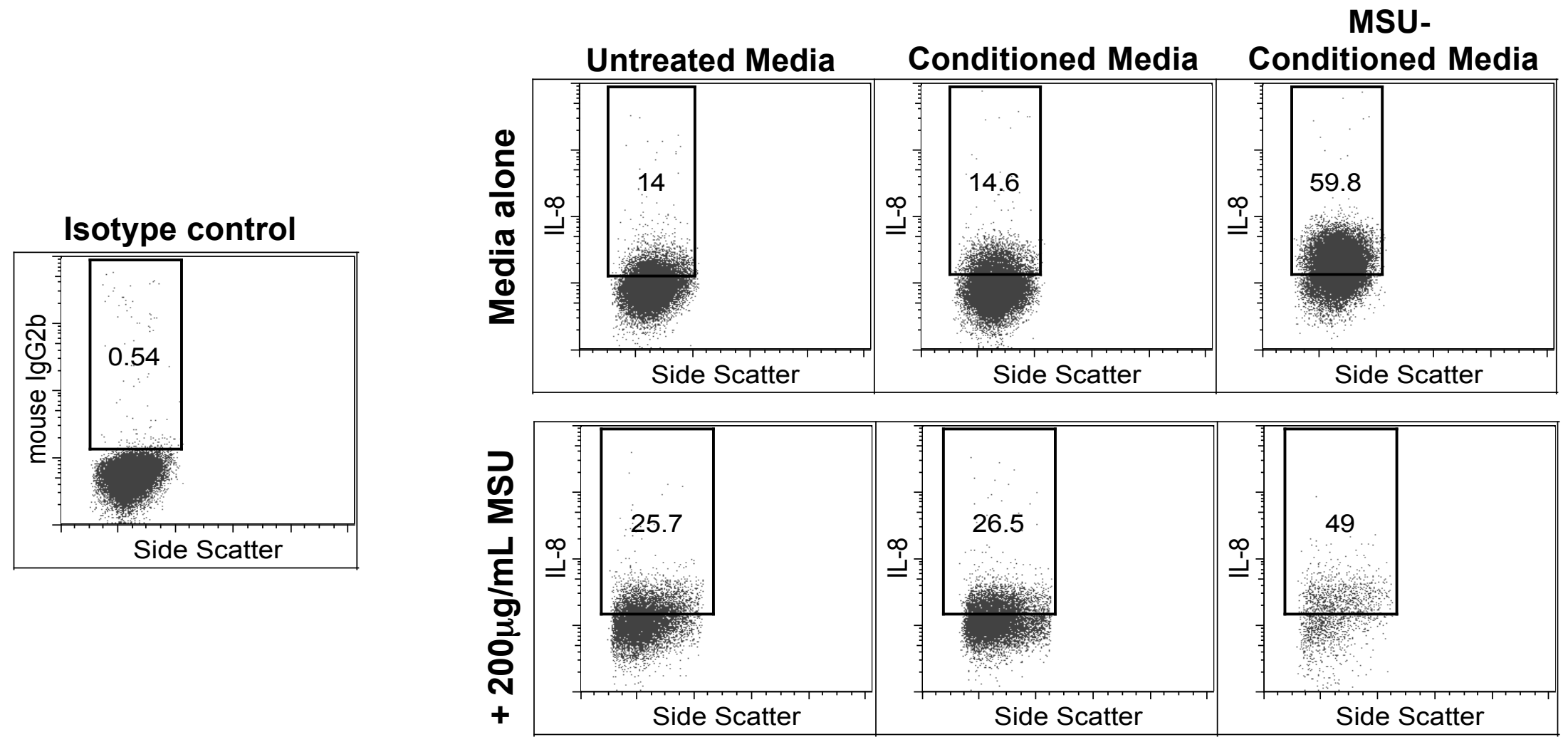
Neutrophils stimulated with MSU-conditioned media alone also produced a much higher level of IL-8 per cell than those stimulated with MSU alone, as shown by the difference in the mean fluorescence intensity (MFI) (Fig. 3.11). Neutrophils receiving both soluble mediators and direct MSU stimulation were difficult to recover but still showed a high proportion of IL- $8^{+}$cells $(49 \%)$ (Fig. 3.10, lower right). Stimulation with MSU provided no additional response in terms of IL-8 production compared to stimulation with soluble mediators alone (Fig. 3.10, top and bottom right).

In summary, soluble mediators from MSU-stimulated cells (represented by MSUconditioned media) exerted a much more potent effect on neutrophil IL-8 production than direct stimulation with MSU.

\subsubsection{MSU-induced soluble mediators prime human neutrophils to produce superoxide}

The effect of the conditioned media on neutrophil superoxide production was examined next. Neutrophils exposed to MSU-conditioned media alone induced a modest, statistically insignificant increase in superoxide production compared with neutrophils cultured in conditioned and unconditioned media (Fig. 3.12, 0mg/mL MSU). Although the maximum response to MSU was the same for all culture media, neutrophils stimulated with MSU in the presence of MSU-conditioned media were sensitised to low concentrations of MSU such that neutrophils produced more superoxide at significantly lower concentrations of MSU than neutrophils cultured in the other media (Fig. 3.12). Hence, in the case of superoxide activity, extracellular signals from MSU stimulated cells appear to play a significant role in neutrophil priming but have no stimulatory effect alone.

\subsubsection{Neutrophil activation and viability in vivo}

The outcomes of the in vitro experiments indicate that if neutrophils are exposed to high levels of pro-inflammatory cytokines prior to contact with MSU crystals, they may infiltrate the site of inflammation in a primed state. Indeed, in a mouse model of MSU-induced peritonitis, the administration of MSU into the peritoneum is associated with the production of pro-inflammatory cytokines both locally, and in 


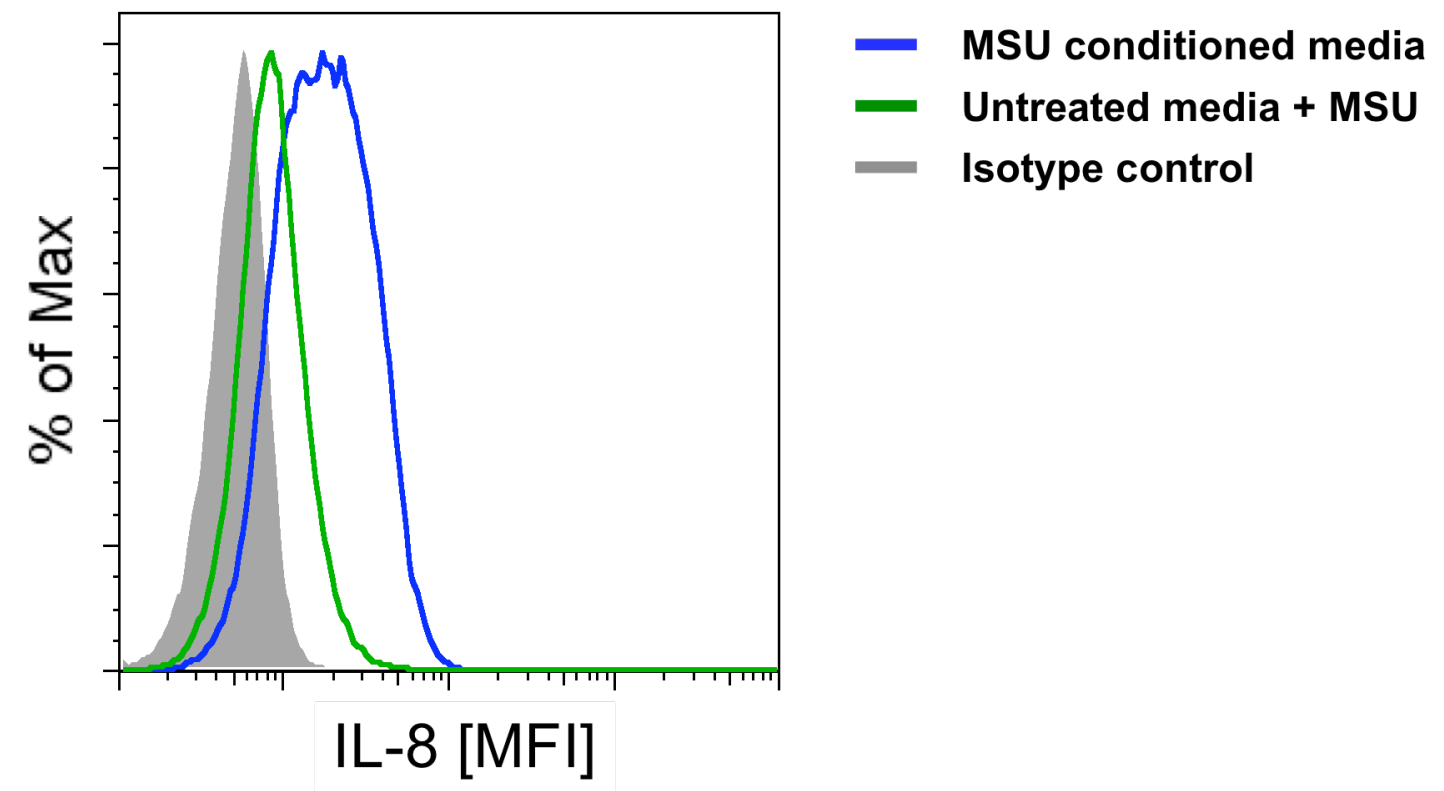

Figure 3.11. Histogram comparing the effect of soluble mediators and MSU on neutrophil IL-8 production. H uman neutrophils were purified from peripheral blood and cultured for $4 \mathrm{~h}\left(5 \times 10^{5}\right.$ cells, $\left.500 \mu \mathrm{L}\right)$ with either MSU-conditioned media, or $200 \mu \mathrm{g} / \mathrm{mL}$ MSU (in unconditioned media). The production of IL-8 by neutrophils was detected by intracellular staining. Results are representative of three separate experiments. 


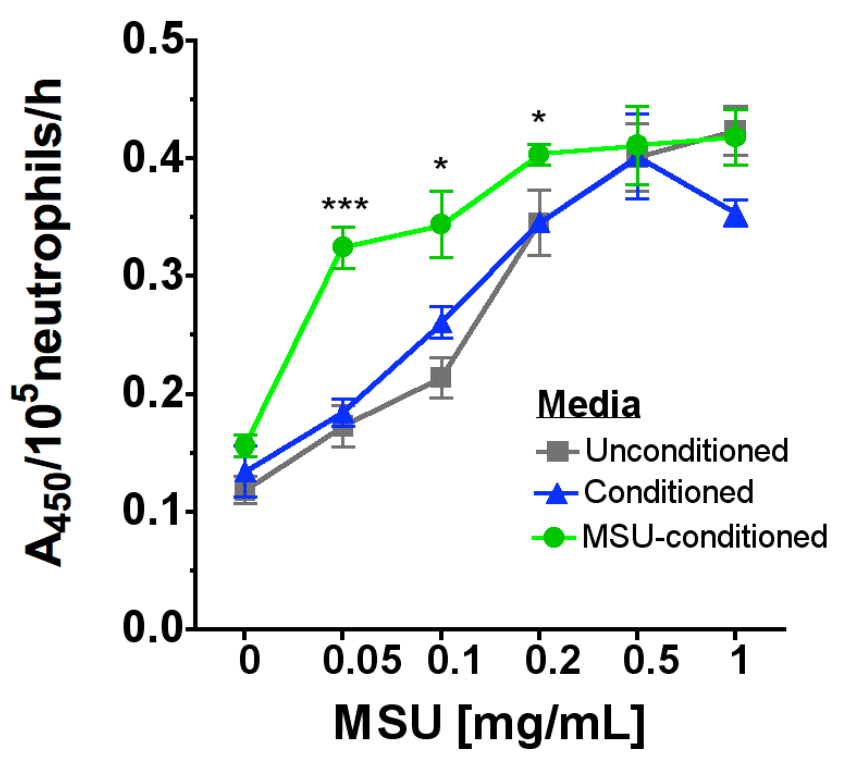

Figure 3.12. The effect of MSU-stimulated soluble mediators on neutrophil superoxide production. Human neutrophils were purified from peripheral blood and cultured for $4 \mathrm{~h}$ in the presence of untreated media (Unconditioned), media conditioned by unstimulated mononuclear cells (Conditioned), or media conditioned by MSU-stimulated mononuclear cells (MSU-conditioned). Neutrophils were then cultured $\left(1.5 \times 10^{5}\right.$ cells, $\left.150 \mu \mathrm{L}\right)$ with various concentrations of MSU in the presence of WST-1 $(200 \mu \mathrm{g} / \mathrm{mL})$. After $1 \mathrm{~h}$, superoxide production was measured by WST-1 dye reduction at absorbance $450 \mathrm{~nm}\left(\mathrm{~A}_{450}\right)$. Measurements were performed in triplicate. Values represent mean \pm S.E.M. ${ }^{*}=P<0.05$ and $* * *=P<0.001$ as determined by Student's $t$-test against the Unconditioned control at the relevant MSU concentration. Results are representative of three separate experiments. 
the serum (Figs. 5.3 and 5.16), which provides two points of exposure to neutrophilstimulating soluble mediators prior to direct contact with MSU. If neutrophils are exposed to these mediators, then they should already show signs of activation once they have infiltrated into the peritoneum.

To test whether neutrophils infiltrating into the peritoneum in response to MSU are activated and produce superoxide, mice were administered with MSU i.p. and four hours later, infiltrating neutrophils were tested for superoxide activity. As a control, a group of mice that had been pre-treated with a subcutaneous injection of colchicine were included (Chia et al., 2008). As a known inhibitor of neutrophil inflammation and a drug used in the treatment of gout, any reduction in neutrophil inflammation observed in colchicine-treated mice would represent a suppression of MSU-induced activation.

MSU crystals induced the recruitment of neutrophils, which was greatly inhibited by colchicine as has been demonstrated previously (Fig. 3.13A) (Chia et al., 2008). More importantly, neutrophils recruited by MSU stimulation produced superoxide in vitro without additional ex vivo stimulation, which was inhibited by colchicine treatment in mice (Fig. 3.13B). These data indicate that neutrophils recruited by MSU are in an activated state while in the peritoneum.

In order to answer the question of whether neutrophils showed improved viability in the peritoneum, the viability of neutrophils over the course of an MSU response in vivo was tested. The rate of neutrophil apoptosis remained steady at around $20 \%$ over the whole duration of the inflammatory response (Fig. 3.14). This was unexpected, as high levels of cytokines in the peritoneum within the first $8 \mathrm{~h}$ and very low cytokines from $8 \mathrm{~h}$ onwards (Fig. 5.3) would have suggested a higher viability in the early stages of recruitment. In addition to this, it would also be anticipated that contact with MSU crystals in the peritoneum would also improve neutrophil viability in the early stages of recruitment. The most likely reason for the stable rate of apoptosis (or viability) observed, is that neutrophils were fully activated by the time they had infiltrated the peritoneum, and as such, $80 \%$ viability may represent the upper limit of neutrophil viability in gout inflammation in vivo. 

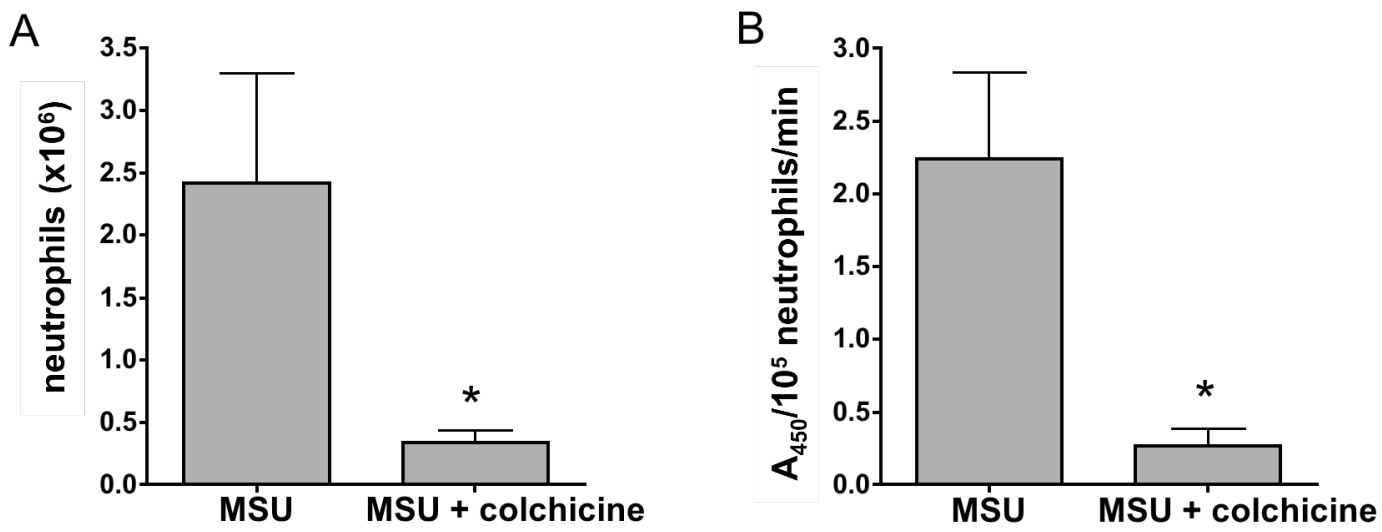

Figure 3.13. Production of superoxide by MSU recruited neutrophils. Mice were treated with an i.p. injection of MSU $(3 \mathrm{mg}, 0.5 \mathrm{~mL}$ PBS) and 4h later, the cells were harvested from the peritoneum by lavage ( $3 \mathrm{~mL}$ PBS). A control group of mice that received a subcutaneous injection of colchicine $(2 \mathrm{mg} / \mathrm{kg})$ prior to MSU administration were also included. (A) Total neutrophils in the peritoneum after $4 \mathrm{~h}$. (B) The rate of superoxide production was measured by WST-1 dye reduction $(200 \mu \mathrm{g} / \mathrm{mL})$ at absorbance $\mathrm{A}_{450}$ over $20 \mathrm{mins}$ with the rate adjusted to per $10^{5}$ neutrophils. Data represented by three mice per group. Values represent mean \pm S.E.M. ${ }^{*}=P<0.05$ as determined by Student's $t$-test. Results are representative of three separate experiments. 


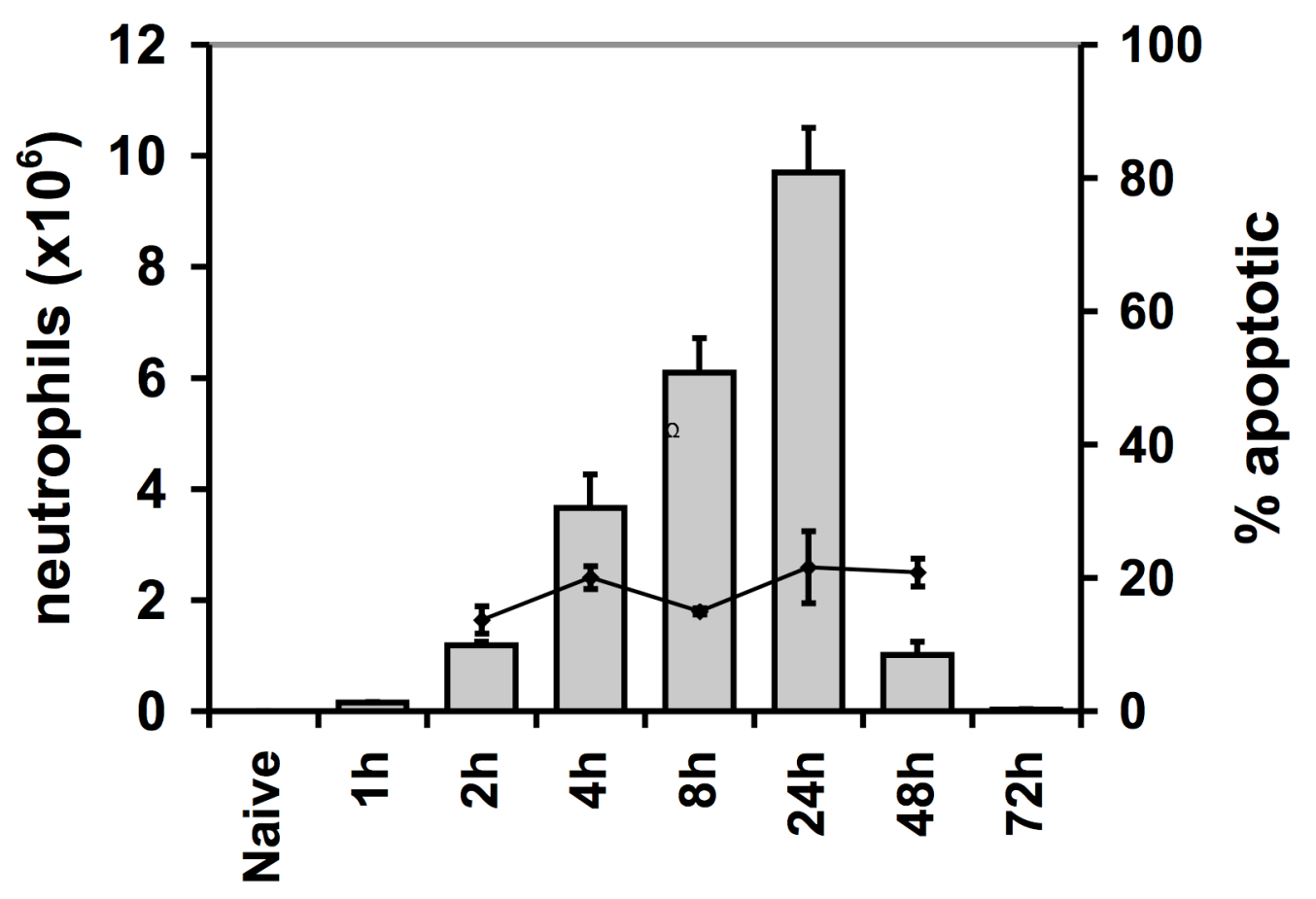

Figure 3.14. Neutrophil viability over the course of the acute gout response. Mice were treated with an i.p. injection of MSU $(3 \mathrm{mg}, 0.5 \mathrm{~mL}$ PBS) and at various times over the course of the response the cells were harvested from the peritoneum by lavage (3mL PBS). Neutrophil numbers (grey bars) were determined by cell count and Diff-Quik staining of cytospin slides. Neutrophil apoptosis (black line) was determined by annexin-V/PI staining of Gr- $1^{+}, F 4 / 80^{-}$cells. $n=3$ mice per group. Values represent mean + S.E.M. 
These in vivo results suggest that MSU-recruited neutrophils are in an activated state in the peritoneum, producing superoxide and are potentially at their limit of viability in vivo.

\subsection{Discussion}

As an important cell in the pathophysiology of gout, neutrophils have been the focus of many prior investigations that have described some of the responses of neutrophils to MSU including IL-8 production, the generation of ROS and an improvement in neutrophil viability. My results elaborate on these prior studies by demonstrating that the induction of these activities can occur independently of MSU contact via interaction with the cellular mediators generated by MSU-induced inflammation.

Cellular mediators were able to cause a delay in apoptosis and induce IL-8 production in neutrophils in the absence of direct contact with MSU crystals, and the level of activation was greater in magnitude than that observed after direct contact with MSU crystals. The results of this study contradict other MSU studies that have assumed that these activities require direct MSU contact. In fact, the presence of both soluble mediators and MSU crystals together provided no further improvement in neutrophil viability or IL-8 production over that achieved by soluble mediator activation alone. This suggests that soluble mediators play a more significant role in neutrophil viability and IL-8 production in gout inflammation than direct MSU contact. Neutrophil viability remained steady at $80 \%$ during an MSU challenge in vivo (Fig. 3.14). This appears to correlate well with the maximum viability of stimulated neutrophils in vitro, which was also $80 \%$ (Fig. 3.8C).

Physical contact with MSU was required for the generation of superoxide by neutrophils. This makes sense at a physiological level, as the premature generation of superoxide would cause unnecessary collateral damage to healthy body tissues if produced while neutrophils are in transit. The requirement of MSU contact would therefore restrict the production of damaging ROS to the site of inflammatory insult. Nevertheless, the requirement of MSU contact for inducing superoxide production did not exclude a role for soluble mediators in superoxide production. Exposure of neutrophils to soluble mediators had a priming effect, causing neutrophils to become 
sensitised to MSU. Although the maximal level of superoxide production of neutrophils did not change, a much lower concentration of MSU was required to reach the maximal level of superoxide production. Hence, soluble mediators affect the intensity of the inflammatory response in the presence of small amounts of MSU.

The priming of neutrophils to MSU-induced superoxide production has been explored in vitro previously, but that study used only purified TNF $\alpha$ and GM-CSF as priming cytokines. The research discussed in this chapter uses inflammatory mediators generated directly from MSU-stimulated mononuclear cells, making the soluble mediators directly relevant to MSU inflammation. I used PBMCs to model the production of soluble mediators by inflammatory cells. In an in vivo response, the source of these cytokines is likely to be MSU-stimulated resident cells. In fact, the generation of cytokines to an in vivo MSU response is explored in a later chapter of this thesis (Chapter 5). Nevertheless, PBMC-generated cytokines are known to be associated with gout inflammation (IL-8, IL-6, TNF $\alpha$, IL-1 $\beta$ ). Further work is still required to dissect the contribution of each cytokine to neutrophil priming as well as IL-8 production and neutrophil viability.

The studies described here further revealed a previously unreported effect of human serum on MSU-induced superoxide production. In the absence of serum, there was little background production of superoxide by neutrophils; however, the addition of very small levels of human serum alone $(<2 \%)$ significantly increased superoxide production. Background superoxide production returned to that observed in the absence of serum when serum levels were increased to $10 \%$. The effect on superoxide did not require autologous serum, suggesting these to be general effects of human serum but not FBS on neutrophil activation. In fact, FBS inhibited background levels of superoxide at all concentrations of serum tested. This suggests that the stimulatory effect of human serum is not a universal feature of all sera. The observed changes in serum-induced superoxide production were partially mediated by proteins that were heat labile $\left(56^{\circ} \mathrm{C}, 30 \mathrm{mins}\right)$, which may include complement proteins that are known to be inactivated by this treatment. It is possible that the response of neutrophils to low serum is a sensing mechanism to indicate movement from the high serum transit environment of the blood to the low serum environment 
of the inflammatory site such as the peritoneum or joint. In this way, a low serum environment may be a non-specific activating trigger for neutrophils to produce superoxide.

There may be two ways to stimulate the production of superoxide from human neutrophils: exposure to a low serum environment; and direct stimulation with MSU. It seems that physiologically this system has "covered its bases" whereby the production of neutrophil superoxide is certain following recruitment to an inflamed tissue. Ultimately, the observed effect is high superoxide output regardless of whether it is serum or MSU mediated.

The effects of serum on MSU-induced neutrophil superoxide imply that the contribution of serum components to neutrophil activation is a novel finding. Previous studies investigating the effects of serum on MSU inflammation used very different approaches. For instance, a previous study by Terkeltaub et al. showed that MSU crystals that had been pre-coated with serum proteins elicited a diminished superoxide response in neutrophils compared with uncoated MSU. This reduced response was due to apolipoprotein B coating the surface of crystals (Terkeltaub et al., 1984). Indeed, this area is not well studied, and as such, further investigations into the effects of serum on neutrophil function to MSU in vivo are required to understand the true mechanisms at play during gouty disease.

The alterations to neutrophil responsiveness by soluble mediators serve as a caveat to studying responses from neutrophils purified from individuals not undergoing an inflammatory response. Neutrophils from healthy individuals have received no priming or activating signals prior to exposure to MSU in vitro whereas neutrophils that have been recruited to a site of inflammation such as a joint will have received inflammatory signals that alter the responsiveness of those neutrophils when they finally make contact with MSU. Hence, neutrophils during gouty attacks and neutrophils under homeostatic conditions represent different starting points as far as a subsequent MSU challenge is concerned. As the use of human peripheral blood neutrophils is a routine practice, it is important to recognise these limitations. 


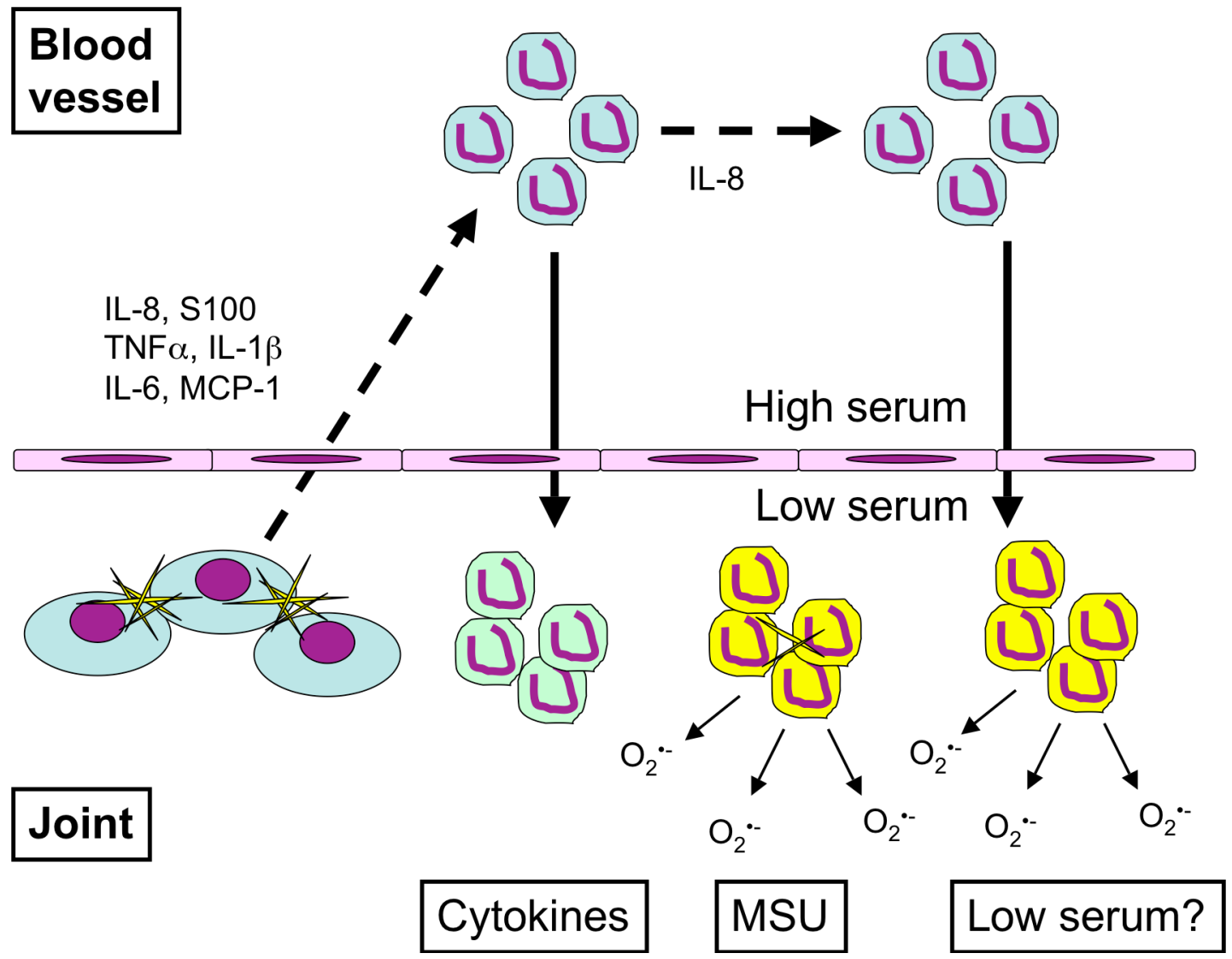

Figure 3.15. Proposed model of neutrophil activation in gout. MSU crystals stimulate local cells in the joint to produce a number of pro-inflammatory cytokines that stimulate the recruitment of neutrophils to produce IL-8 (augmenting further neutrophil recruitment). Exposure to cytokines in the joint also leads to a delay in neutrophil apoptosis and to neutrophils being primed to produce superoxide (green). Neutrophils infiltrating the joint space receive at least two additional stimuli to produce superoxide: sensitised neutrophils produce superoxide on contact with MSU (yellow), and/or they are stimulated to produce superoxide as a result of moving to a potentially low serum environment (yellow). 
The results presented in this chapter suggest an alternative model of neutrophil activation in gout that incorporates the effects of serum and soluble mediators as depicted in Figure 3.15. After the formation of MSU crystals, resident cells produce cytokines and chemokines that stimulate neutrophils and cause them to delay apoptosis, produce IL-8 and become primed to produce superoxide, all before making contact with MSU. These factors work together to amplify neutrophil numbers through recruitment (e.g. through IL-8 related activites) and allow their accumulation (due to an extended lifespan). Neutrophils recruited to the joint produce large amounts of superoxide, either on contact with small quantities of MSU, and/or producing superoxide spontaneously as a result of movement from the high serum environment of the blood to the potentially low serum environment of the joint. Together these events allow for the neutrophil rich inflammatory environment observed during an MSU inflammatory attack.

In summary, these results indicate that activation of neutrophils in gout is not simply initiated by contact of neutrophils with MSU crystals: activation begins prior to MSU contact through soluble mediators and low serum effects. Soluble mediators stimulate IL-8 production and cause a delay in neutrophil apoptosis. While they do not stimulate the release of superoxide, they heavily affect the magnitude of the response by exerting a priming effect. Low human serum conditions are able to stimulate the production of large amounts of superoxide even in the absence of MSU crystals. These findings highlight previously unreported pathways of neutrophil activation in gout that do not require contact with MSU and are likely to play a significant role in gout inflammation. 
Chapter 4

Inhibiting neutrophil inflammation using sesquiterpene dialdehydes 


\section{Chapter 4}

\section{Inhibiting neutrophil inflammation using sesquiterpene dialdehydes}

\subsection{Introduction}

Polygodial is a sesquiterpene dialdehyde that is an active component in Horopito (Pseudowintera colorata), a native medicinal plant in New Zealand; and in Winter's Bark (Drymis winteri), a medicinal plant used in South America (Morton, 1981, McCallion et al., 1982). These plants have been used traditionally to treat a number of symptoms including stomach pain, allergy, bronchitis and inflammation.

A number of studies have explored the antifeedant (Kubo and Ganjian, 1981), antifungal (Kubo et al., 2001 ), and antinociceptive properties of polygodial (Mendes et al., 2000); however, only one study has extensively explored polygodial as an anti-inflammatory. In this study, polygodial inhibited both swelling and leukocyte infiltration in mouse models of oedema and pleurisy using a number of proinflammatory stimuli (da Cunha et al., 2001). The broad range of anti-inflammatory activities highlighted polygodial as a promising compound for further investigation and potential development as an anti-inflammatory drug with broad applicability. To date the effect of polygodial on cellular function in the context of inflammation or acute gouty inflammation has not been investigated.

\subsubsection{Neutrophil infiltration and superoxide production in gouty inflammation}

Neutrophil recruitment is a key feature of gouty inflammation (Phelps and McCarty, 1966). Once recruited, neutrophils become activated and produce superoxide that is converted to other more destructive ROS (Section 1.10.7.4). Therefore, I investigated the potential of polygodial to block MSU-induced superoxide production by neutrophils as a novel therapeutic approach for treating gout, with possible application to other neutrophil-driven inflammatory conditions. To gain insight into the structural features required for inhibition, the anti-inflammatory activity of polygodial was compared against a group of structurally-related 


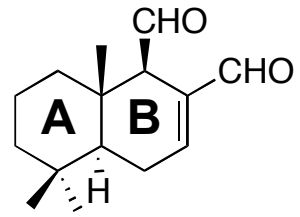

\section{Compound 1}

(polygodial)<smiles>C=C1[C@H](C)C[C@@H](O)[C@]2(C)[C@H](C=O)C=C[C@H](C=O)[C@H]12</smiles>

Compound 2<smiles>O=CC1=CCCCC1C=O</smiles>

Compound 5<smiles>C=C1C(C)CC(OC(=O)/C=C/c2ccccc2)C2(C)[C@@H](C=O)C(C=O)=CC[C@H]12</smiles>

Compound 3<smiles>O=Cc1ccccc1C=O</smiles>

Compound 6

(Phthalaldehyde)<smiles>C=C1C(C)[C@@H](O)CC(OC(=O)/C=C/c2ccccc2)C2(C)[C@H](C=O)C(C=O)C[C@H](O)[C@H]12</smiles>

Compound 4<smiles>C=C1C(C)CC(OC(=O)/C=C/c2ccccc2)[C@@]2(C)C1CC=C1C(OC)OC(OC)C12</smiles>

Compound 7

Figure 4.1 Sesquiterpene compound structures 
compounds, shown in Figure 4.1. The outcomes of this study provide basic information on the structure-function relationship of these compounds with a view to develop a more potent compound that can be used in neutrophil-based inflammatory diseases such as gout.

\subsection{Results}

\subsubsection{Inhibition of neutrophil superoxide production by sesquiterpene dialdehydes}

Neutrophils can be activated to produce superoxide by a variety of different inflammatory stimuli. To determine the ability of the sesquiterpenes to inhibit neutrophil superoxide production, the compounds were tested in human neutrophil cultures treated with either phorbol myristate acetate (PMA) or the gout-causing inflammatory stimulus MSU.

PMA activates superoxide production by directly activating intracellular protein kinase $\mathrm{C}$, thus representing a non-specific induction of superoxide production (Blumberg, 1988). PMA stimulation therefore provides information on the antiinflammatory capacity of the compounds in general. MSU activation of neutrophils, although not clearly understood, is known to be associated with microtubule stabilisation (Chia et al., 2008) and was used to provide a more physiologically relevant measure of superoxide production and inhibition.

\subsubsection{Structure-activity related inhibition of PMA-induced superoxide production}

As shown in Table 4.1, Polygodial (Compound 1) exhibited a concentrationdependent inhibition of superoxide production by neutrophils stimulated with PMA with an $\mathrm{IC}_{50}$ value of $0.16 \mu \mathrm{M}$. The introduction of methyl and alcohol functionalties to ring $\mathrm{A}$ of the polygodial structure and exchanging the dimethyl in ring $\mathrm{A}$ for an ethylene group (Compound 2) caused only a minor decrease in anti-inflammatory activity. Notably, the presence of a large cinnamate ester group (Compound 3) resulted in a 27-fold decrease in the anti-inflammatory activity compared to Compound 2. This suggested that steric hindrance from the large ester group around 


\section{$\mathrm{IC}_{50}$ values $(\mu \mathrm{M})$ for $\mathrm{O}_{2}^{-}$production}

\begin{tabular}{|c|c|c|}
\hline Compound & PMA & MSU \\
\hline 1 & $0.16 \pm 0.13$ & $0.78 \pm 0.53$ \\
2 & $0.39 \pm 0.07$ & $0.82 \pm 0.24$ \\
3 & $4.37 \pm 1.39$ & $2.00 \pm 0.58$ \\
4 & $0.50 \pm 0.22$ & $1.07 \pm 0.04$ \\
5 & $3.53 \pm 2.56$ & $7.49 \pm 2.31$ \\
6 & $3.68 \pm 1.21$ & $5.84 \pm 1.72$ \\
7 & $406 \pm 23.7$ & n.d. \\
\hline
\end{tabular}

Table 4.1 IC IC $_{50}$ values of sesquiterpene compounds based on PMA- and MSUstimulated superoxide production by neutrophils. Human neutrophils were purified from peripheral blood, suspended in HBSS $\left(10^{5}\right.$ neutrophils/well; $100 \mu \mathrm{L}$ HBSS) and incubated in the presence of the sesquiterpene compounds. Neutrophils were stimulated with $0.2 \mu \mathrm{g} / \mathrm{mL}$ PMA for 30 minutes or with $200 \mu \mathrm{g} / \mathrm{mL}$ MSU for $1 \mathrm{~h}$ and the production of superoxide measured by WST-1 dye reduction. $\mathrm{IC}_{50}$ values were calculated from the dose response curves. Results are a combination of $\mathrm{IC}_{50}$ values from three separate experiments, where PMA $\mathrm{IC}_{50}$ values are a combination of 2 experiments from E. Chia and one from the W. Martin; and MSU $\mathrm{IC}_{50}$ values were by experiments from the W. Martin alone. Values represent mean \pm SEM. 
the dialdehyde functionalities may have been the cause for loss of activity. Surprisingly, loss of inhibition observed for Compound 3 was 'rescued' by the introduction of an alcohol group to ring B (Compound 4) suggesting that the addition of the alcohol group overcomes the steric hindrance of the cinnamate group possibly by providing an alternative/additional active site or altering the conformation of the cinnamate group to free up the reactive aldehyde groups.

The importance of dialdehyde functionalities for activity was tested by using paxidal dimethyl acetal (Compound 7). Loss of the dialdehyde functionalities resulted in a 93-fold decrease in anti-inflammatory activity compared to Compound 3 confirming that the majority of the inhibitory activity of the compounds was associated with the dialdehyde groups.

The presence of the ring dilaldehydes alone was shown to be sufficient to inhibit neutrophil superoxide production. The absence of ring A from (Compound 5) resulted in a 22 fold loss of anti-inflammatory activity compared to polygodial and 10 fold loss compared to Compound 2. A similar loss in activity was also observed for the aromatic dialdehyde (Compound 6). Together these data indicated that both the dialdehyde and the A ring contribute to the inhibitory effect of this group of compounds.

\subsubsection{Structure-activity related inhibition of MSU-induced superoxide production}

After confirming that dialdehyde-containing compounds suppressed superoxide production by neutrophils stimulated with PMA, I sought to determine if these compounds could also inhibit neutrophil superoxide production induced by MSU crystals. As shown in Table 4.1 polygodial was less effective at inhibiting MSUinduced compared with PMA-induced superoxide production. In general all of the compounds tested were only slightly weaker inhibitors of MSU-induced superoxide production compared to PMA, the one exception being Compound 3, which exhibited slightly better inhibition of MSU-induced inflammation. The comparable 
pattern of inhibitory activity observed for PMA and MSU-induced superoxide production indicated that the compounds were likely blocking superoxide production via the same, as yet unknown, pathway.

\subsubsection{Cytotoxic activity of sesquiterpene dialdehydes}

To exclude the possibility that the anti-inflammatory effect exhibited by the compounds was not simply the result of cell death, the active compounds were tested for possible cytotoxic effects on neutrophils. Isolated human neutrophils were treated with the compounds for 30 minutes, and cell viability determined by trypan blue exclusion. With the exception of Compound 3, none of the compounds tested were cytotoxic at the concentrations required for anti-inflammatory activity indicating that the inhibitory activity observed was not due to cytotoxicity (Fig. 4.2).

Although no cell death occurred at concentrations required for anti-inflammatory activity, some cell death was observed at higher concentrations. To determine which pathway was being activated, apoptosis or necrosis, the highest compound dose was used from the above 30 minute assay to induce cell death in neutrophils, with an extended length of exposure to four hours to allow for the expression of markers to indicate the mode of cell death. Cells were then stained with annexin-V/PI. As shown in Figure 4.3, exposure to $10 \mu \mathrm{M}$ of each compound caused an increase in the annexin- $\mathrm{V}^{+} / \mathrm{PI}^{-}$cells, showing that the compounds induced apoptosis, rather than necrosis, in neutrophils.

After four hours, all of the compounds triggered concentration-dependent apoptosis of neutrophils with the concentrations required to observe $50 \%$ cell death (Toxic concentration $50 \%, \mathrm{TC}_{50}$ ) are shown with $\mathrm{IC}_{50}$ values in Table 4.2. Interestingly, lower anti-inflammatory activity was associated with high cell toxicity (Compounds 3, 5 and 6), whereas compounds with high anti-inflammatory activity displayed significantly lower toxicity (Compounds 1,2 and 4). This is illustrated when $\mathrm{IC}_{50}$ values are shown as a ratio of $\mathrm{TC}_{50}$ values in which high ratios indicated high antiinflammatory activity (Table 4.2). These results indicate that the structural 

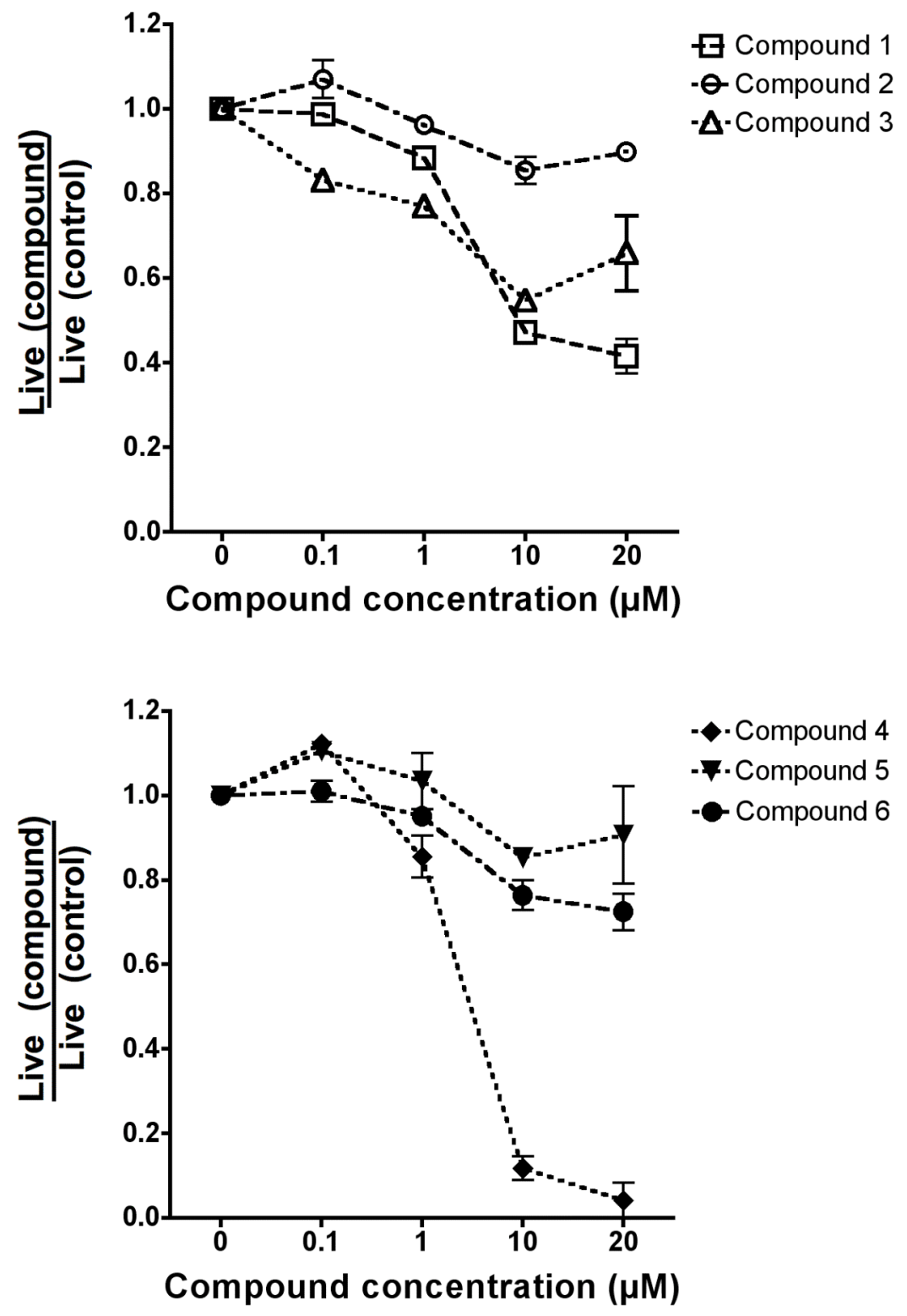

Figure 4.2 The effect of sesquiterpene compounds on neutrophil viability after 30 minutes. Human neutrophils were isolated from peripheral blood and cultured in HBSS $\left(5 \times 10^{5}\right.$ cells, $\left.500 \mu \mathrm{L}\right)$ in the presence of various concentrations $(0.1$ to $20 \mu \mathrm{M})$ of the sesquiterpene compounds. After 30 mins neutrophil viability was determined by trypan blue exclusion. Values were determined from duplicates. Results represent mean \pm S.E.M. Results are representative of three separate experiments. 


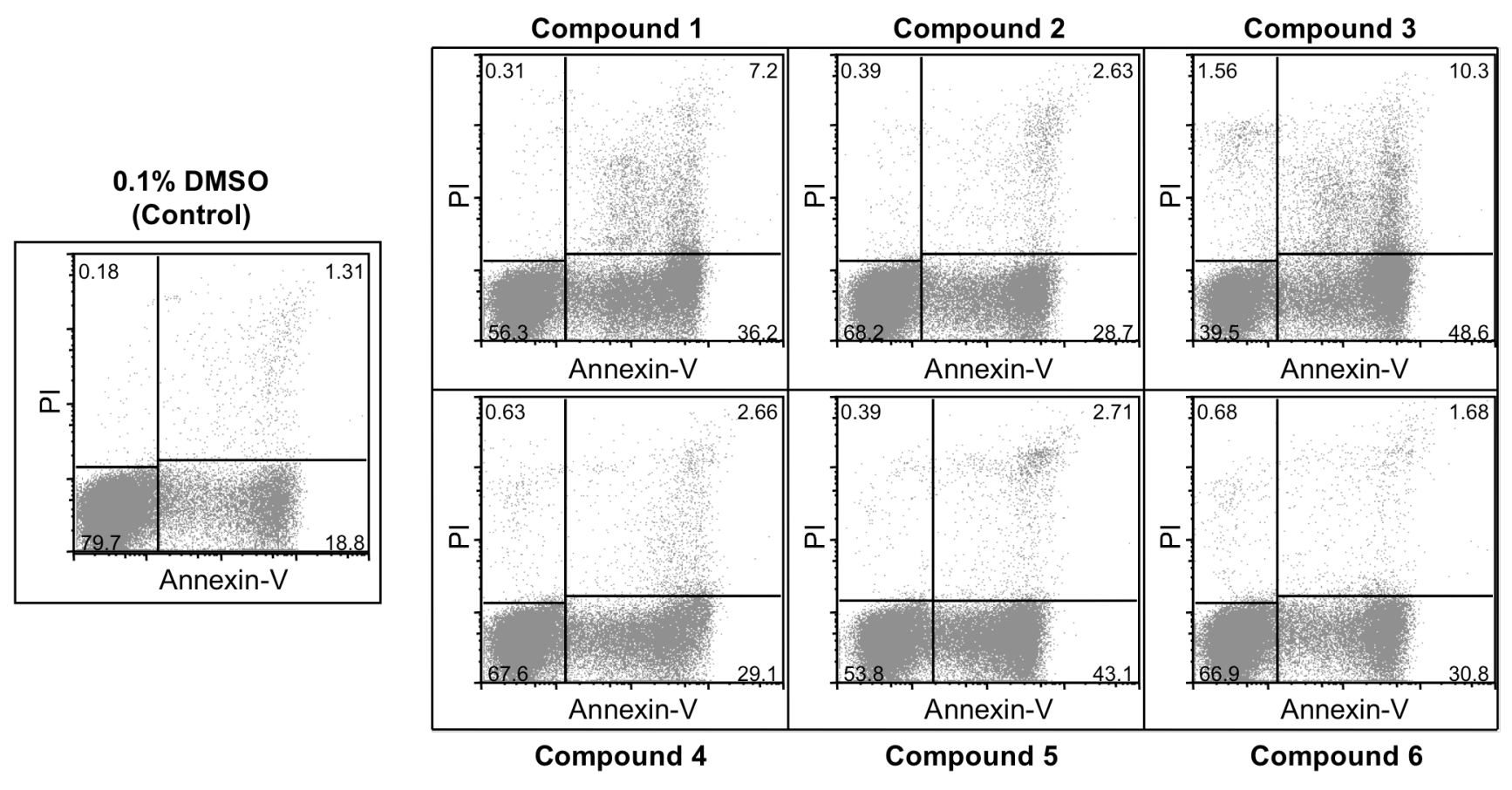

Figure 4.3 The effect of sesquiterpene compounds on neutrophil apoptosis. Human neutrophils were isolated from peripheral blood and cultured in RPMI-1640 (10\% human serum $)\left(5 \times 10^{5}\right.$ cells, $\left.500 \mu \mathrm{L}\right)$ in the presence of sesquiterpene compounds $(10 \mu \mathrm{M})$. After $4 \mathrm{~h}$ neutrophil viability was determined by Annexin-V/PI staining. Results are representative of three separate experiments. 


\begin{tabular}{|c|c|c|c|}
\hline Compound & $\begin{array}{c}\mathrm{IC}_{\mathbf{5 0}}[\mathrm{PMA}] \\
\boldsymbol{\mu M}\end{array}$ & $\mathrm{TC}_{\mathbf{5 0}}$ & {$\left[\mathrm{TC}_{\mathbf{5 0}} / \mathrm{IC}_{\mathbf{5 0}}\right]$} \\
\hline $\mathbf{1}$ & 0.16 & $>20$ & $>125$ \\
$\mathbf{2}$ & 0.39 & 17.5 & $\mathbf{4 5}$ \\
$\mathbf{3}$ & 4.37 & 10 & $\mathbf{2}$ \\
$\mathbf{4}$ & 0.5 & $>20$ & $>\mathbf{4 0}$ \\
$\mathbf{5}$ & 3.53 & 14 & $\mathbf{4}$ \\
$\mathbf{6}$ & 3.68 & 14.5 & $\mathbf{4}$ \\
\hline
\end{tabular}

\begin{tabular}{|c|c|c|c|}
\hline Compound & $\begin{array}{c}\mathrm{IC}_{50}[\mathrm{MSU}] \\
\boldsymbol{\mu M}\end{array}$ & $\mathrm{TC}_{\mathbf{5 0}}$ & {$\left[\mathrm{TC}_{\mathbf{5 0}} / \mathrm{IC}_{\mathbf{5 0}}\right]$} \\
\hline $\mathbf{1}$ & 0.78 & $>20$ & $>26$ \\
$\mathbf{2}$ & 0.82 & 17.5 & $\mathbf{2 1}$ \\
$\mathbf{3}$ & 2 & 10 & $\mathbf{5}$ \\
$\mathbf{4}$ & 1.07 & $>20$ & $>19$ \\
$\mathbf{5}$ & 7.49 & 14 & $\mathbf{2}$ \\
$\mathbf{6}$ & 5.84 & 14.5 & $\mathbf{2}$ \\
\hline
\end{tabular}

Table 4.2 A comparison of sesquiterpene $\mathrm{IC}_{50}$ values for neutrophil superoxide production with sesquiterpene $\mathbf{T C}_{50}$ values. $\left[\mathrm{TC}_{50} / \mathrm{IC}_{50}\right]$ values represent a ratio between the $\mathrm{TC}_{50}\left(50 \%\right.$ Toxicity Concentration) and $\mathrm{IC}_{50}(50 \%$ Inhibition Concentration) values, with high values representing high anti-inflammatory activity and low toxicity. The values shown in blue represent the minimum value that could be calculated from the $\mathrm{TC}_{50}$ values available. 
components that improve anti-inflammatory activity may also abrogate cytotoxic effects.

In summary, the data presented here confirm that the sesquiterpene dialdehyde compounds inhibit superoxide production rather than induce cell death. In addition, the presence of the dimethyl functionality on Polygodial on ring A, and the presence of an alcohol group on either ring A (Compound 2) or ring B (Compound 4) appears not only to increase anti-inflammatory activity, but lower cytotoxicity as well.

\subsubsection{Inhibition of neutrophil superoxide production in vivo}

Acute gouty arthritis is characterised by the recruitment and activation of neutrophils, and the production of superoxide, in response to the known inflammatory stimulus MSU. A peritoneal model of gouty arthritis was used to determine whether the in vitro anti-inflammatory activity of the compounds translated into an inhibitory effect in vivo. Compounds polygodial, Compound 2 and Compound 4 were selected for in vivo testing on the basis of their high antiinflammatory activity and low cytotoxic activity in vitro (Table 4.2). These compounds were tested in vivo in a peritoneal model of gouty inflammation and assessed for their efficacy at inhibiting both neutrophil recruitment and neutrophil superoxide production by infiltrating neutrophils.

Polygodial has been shown previously by others in our lab to inhibit both neutrophil infiltration and superoxide production in vivo at a dose of $25.6 \mu \mathrm{mol} / \mathrm{kg}$. Therefore, polygodial was used as a positive control to compare the activity of Compound 2 and Compound 4. Compound 4 exhibited dose-dependent inhibition of both neutrophil infiltration (Fig. 4.4A) and superoxide production (Fig. 4.4C) in vivo. Despite a clear trend, Compound 2 did not significantly inhibit neutrophil infiltration (Fig. 4.4B), however, Compound 2 exhibited significant inhibition of superoxide production (Fig. 4.4D). 
A

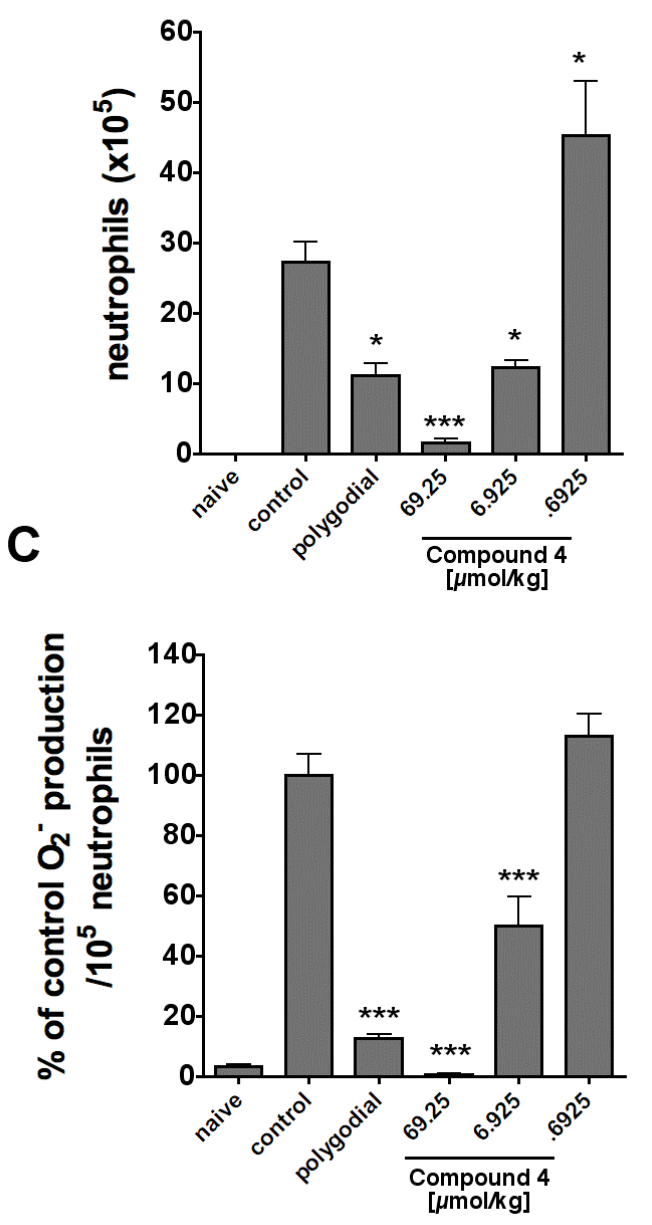

B
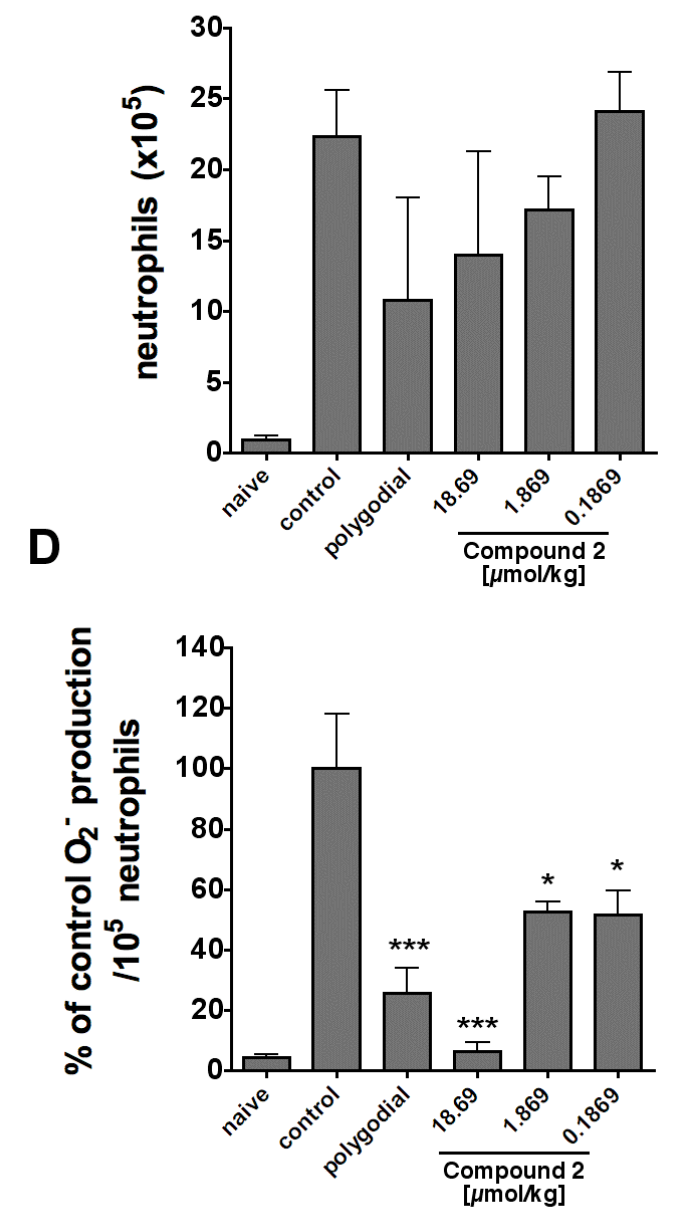

Figure 4.4 The effect of sesquiterpene compounds on neutrophil inflammation in vivo. Mice were treated with an i.p. injection of the sesquiterpene compounds, $25.6 \mu \mathrm{mol} / \mathrm{kg}$ of polygodial, or with $50 \mu \mathrm{L}$ of DMSO (control) and then administered with an i.p. injection of MSU $(3 \mathrm{mg}, 0.5 \mathrm{~mL})$. 4h later the peritoneal exudate cells were collected by lavage (3mL PBS, heparin). Cell counts and cytospin differentials were used to determine neutrophil infiltration of mice treated with (A) Compound 4 and (B) Compound 2. WST-1 dye reduction was followed over 20 mins at absorbance $450 \mathrm{~nm}$ as a measure of superoxide production by neutrophils from mice treated with (C) Compound 4 and (D) Compound 2. Mice that received no compound nor MSU (naïve) were included as negative controls. Measurements are the result of five mice per group. Values represent mean + S.E.M. $*=P<0.05$ and $* * *=P<0.001$ compared with the control group as determined by Tukey multiple comparisons test. Results are representative of at least two separate experiments. Experiments carried out by E. Chia and figure produced by the W. Martin. 
These in vivo studies showed that Compound 2 and Compound 4 had comparable or greater anti-inflammatory activity than polygodial in vivo and of the three compounds tested, Compound 4 had the greatest effect on neutrophil infiltration (Fig. 4.4B), while polygodial and Compound 2 had similarly high inhibitory activity on superoxide production at doses near $20 \mu \mathrm{mol} / \mathrm{kg}$ (Fig. 4.4C).

\subsubsection{Additional anti-inflammatory activities of sesquiterpene dialadehyde compounds}

One of the interesting findings from the in vivo experiments was the ability of the anti-inflammatory compounds to block MSU-induced neutrophil recruitment. Although polygodial has previously been shown to inhibit leukoctye recruitment in pleurisy models there has been no work undertaken to determine the mechanism for this inhibitory effect. Therefore, I sought to explore whether the compounds were able to affect the function of other cells known to be involved in neutrophil recruitment in MSU-induced inflammation.

\subsubsection{Sesquiterpene dialdehydes compounds inhibit superoxide production by endothelial cells}

Superoxide can be produced by a variety of cells that utilise different isoforms of NADPH oxidase (Nox 1-4) depending on the cell and tissue type and the size of the response required. For example the neutrophil isoform of NADPH oxidase, otherwise known as Nox2, is responsible for the production of high levels of superoxide required for host defense (Section 3.1). However, other isoforms of NADPH oxidase are known to produce much lower levels of superoxide. Vascular endothelial cells express the NADPH oxidase isofoms Nox4, which, instead of producing superoxide as an anti-microbial molecule, produce superoxide that functions as an intracellular signal (Ago et al., 2004). Inhibition of NADPH oxidase on vascular endothelial cells has been shown to inhibit the adherence of leukocytes to endothelial cells in culture, thereby blocking the recruitment of leukocytes in vivo. MSU crystals have been shown to stimulate the production of superoxide from endothelial cells (Falasca et al., 1993), therefore, the inhibition of vascular isoforms 
of NADPH oxidase during an inflammatory response could be contributing to the inhibition of neutrophil recruitment by the compounds in vivo.

To determine if the compounds were able to inhibit superoxide production by epithelial cells, luminol chemiluminescence was used to measure NADPH oxidase activity in human vascular endothelial cell (HUVEC) cultures. Superoxide production was stimulated in HUVECs by reducing the amount of serum in the culture medium to $0.5 \%$. This led to the production of large amounts superoxide compared with the normal culture conditions of $10 \%$ serum (Fig. $4.5 \mathrm{~A}$ ). To confirm that the production of superoxide was dependent on NADPH oxidase, HUVECs in $0.5 \%$ serum were cultured with the inhibitor diphenyleneiodonium sulfate (DPI). DPI is an inhibitor of flavin-containing enzymes such as NADPH oxidase, but does not inhibit the activity of non-flavin containing enzymes capable of ROS generation, such as xanthine oxidase (Cross and Jones, 1986). DPI inhibited superoxide production in a concentration-dependent manner confirming that the production of superoxide by HUVECs was likely due to NADPH oxidase activity, rather than other ROS sources (Fig. 4.5B).

All of the compounds inhibited the production of superoxide from HUVEC cells with $\mathrm{IC}_{50}$ values ranging from 2.5 to $8.4 \mu \mathrm{M}$ (Fig. 4.6). Similar to the inhibition of neutrophil NADPH oxidase, Compounds 2 and 4 showed the highest inhibitory activity. This result indicated that the inhibition of superoxide production by vascular cells may be a possible mechanism for the inhibition of neutrophil infiltration in vivo. However, a higher specificity for neutrophil NADPH oxidase is indicated when the $\mathrm{IC}_{50}$ values of HUVECs are compared with those of neutrophils, the high ratio showing that a higher concentration of compound is required to inhibit the HUVEC oxidase (Table 4.3) 

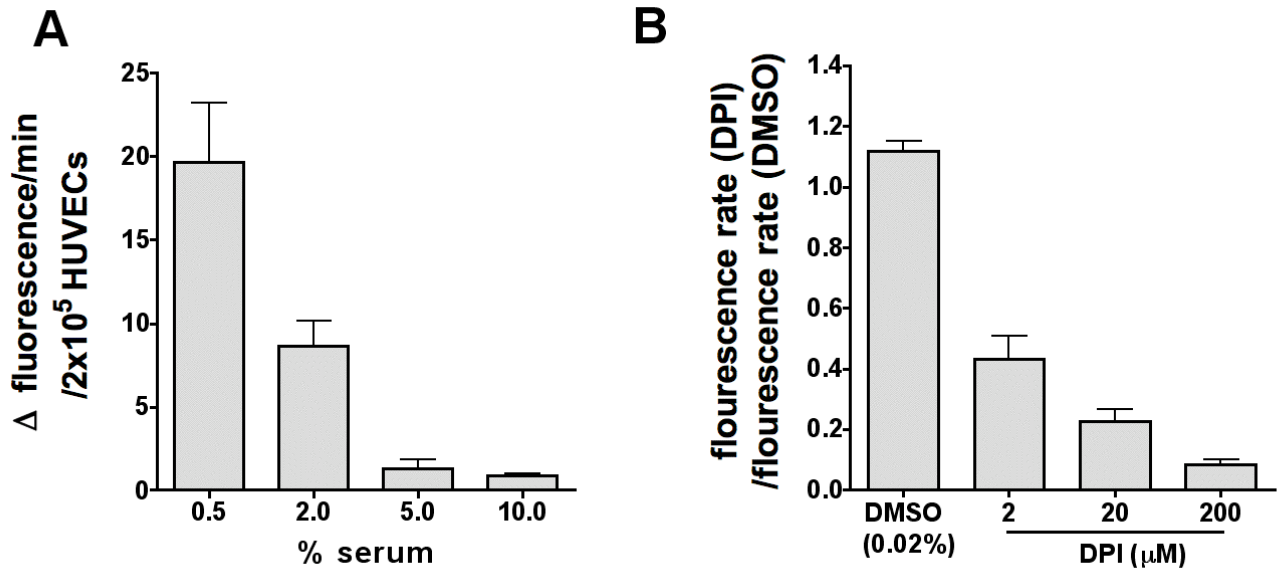

Figure 4.5 The effect of serum on superoxide production from HUVECs. A. HUVECs were cultured in the presence of various concentrations of fetal calf serum and the production of superoxide measured by luminol enhanced chemiluminescence. B. HUVECs were cultured in the presence of $0.5 \%$ serum in the presence of the NADPH oxidase inhibitor DPI. Measurements were performed in triplicate. Values represent mean + S.E.M. Results are representative of three separate experiments. 


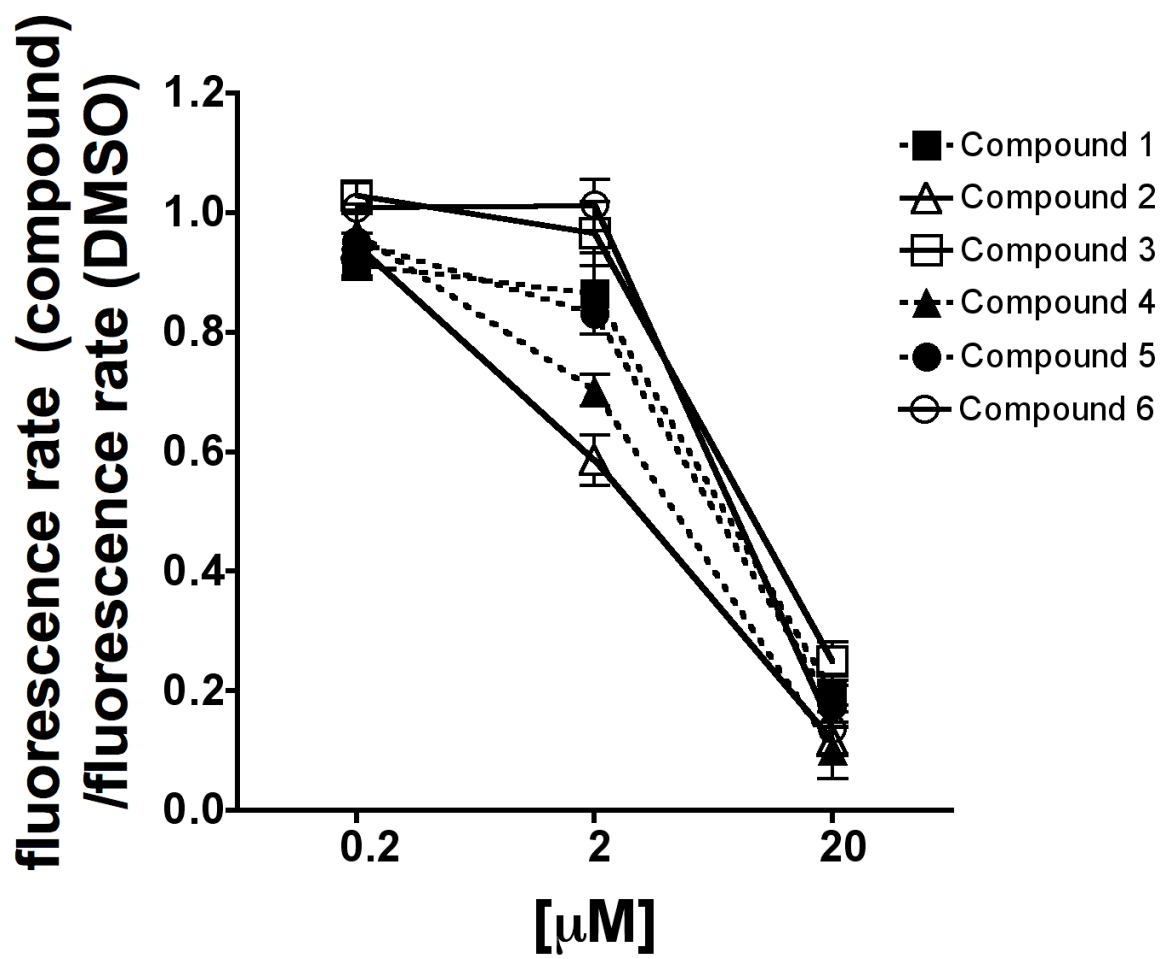

Figure 4.6 The effect of sesquiterpene compounds on superoxide production by HUVECs. HUVECs were cultured in $0.5 \%$ serum in the presence of sesquiterpene compounds and the production of superoxide measured by luminol enhanced chemiluminescence. Measurements were performed in triplicate. Values represent mean + S.E.M. Results are representative of two separate experiments. 


\begin{tabular}{|c|c|c|c|}
\hline \multirow{2}{*}{ Compound } & \multirow{2}{*}{$\begin{array}{l}\text { HUVEC } \\
\text { IC }_{50}(\mu \mathrm{M}) \\
\end{array}$} & \multicolumn{2}{|c|}{ HUVEC IC $_{50} /$ Neutrophil IC $_{50}$} \\
\hline & & $\mathrm{IC}_{50} / \mathrm{IC}_{50}$ PMA & $\mathrm{IC}_{50} / \mathrm{IC}_{50} \mathrm{MSU}$ \\
\hline$\overline{1}$ & 6.5 & 41.9 & 8.3 \\
\hline 2 & 2.5 & 6.4 & 3.1 \\
\hline 3 & 8.4 & 1.9 & 4.2 \\
\hline 4 & 4.1 & 8.2 & 3.8 \\
\hline 5 & 5.2 & 1.5 & 0.7 \\
\hline 6 & 7.5 & 2.0 & 1.3 \\
\hline
\end{tabular}

Table 4.3 Comparison of $\mathrm{IC}_{50}$ values of the sesquiterpene compounds from HUVEC superoxide production, with $\mathrm{IC}_{50}$ values from PMA- and MSUstimulated neutrophil production. 


\subsubsection{Sesquiterpene dialdehyde compounds inhibit proinflammatory} cytokine production in MSU-stimulated macrophages

Macrophages are important inflammatory cells that produce large amounts of cytokines and chemokines in response to inflammatory stimuli (Section 1.10.11). As discussed in Chapter 3, inflammatory mediators play a key role in the recruitment and activation of neutrophils in MSU-induced inflammation. Therefore, I asked whether these compounds could inhibit MSU-induced macrophage activation that could contribute to the inhibition of neutrophil infiltration in vivo. To determine if the compounds were able to inhibit macrophages stimulated with MSU, RAW 264.7 macrophage cells were exposed to MSU in the presence of the compounds, and the cytokines TNF $\alpha$ and IL-6 used as measurements of inflammation.

Due to poor aqueous solubility the compounds had to be dissolved in the organic solvent dimethyl sulfoxide (DMSO). Possible non-specific effects of DMSO upon RAW 264.7 cells were determined by activating RAW 264.7 cells with MSU in the presence of different concentrations of DMSO. Concentrations of DMSO above $0.0125 \%$ inhibited MSU-induced TNF $\alpha$ production, whereas IL-6 production was unaffected by concentrations up to $0.05 \%$ (Fig. 4.7). To avoid complications with higher DMSO levels, compounds were dissolved in 0.01\% DMSO which, due to low compound solubilities, restricted the compound concentrations in the assay to $1 \mu \mathrm{M}$.

As shown in Figure 4.8A, only Compound 3 and Compound 6 inhibited TNF $\alpha$ production by RAW 264.7 cells in vitro, whereas all the compounds, except Compound 6, inhibited IL-6 production to some extent, with Compound 2 exhibiting the greatest inhibitory effect (Fig. 4.8B). Although these compounds appear to alter the response of macrophages to MSU there was no obvious dose-dependent pattern of inhibition. As a result it is impossible to conclude whether or not the inhibition of MSU-induced neutrophil infiltration in vivo by polygodial and Compounds 2 and 4 (Fig. 4.4) might be linked to the inhibition of MSU-induced activation of macrophages. 


\subsection{Summary and Conclusion}

Polygodial has been shown to exert an inflammatory effect in a number of models of inflammation although a cellular basis for the anti-inflammatory activity was not explored in these models. The results presented in this chapter show that neutrophils are a target of polygodial anti-inflammatory activity and that this activity is also observed in additional sesquiterpene dialdehydes in this study. The sesquiterpene dialdehydes inhibit both PMA and MSU induced superoxide production in vitro. This inhibitory activity was largely dependent on the dialdehyde groups but was further enhanced with the presence of ring hydroxyl groups. Interestingly, removal of ring hydroxyl groups caused an increase in cytotoxicity suggesting that the hydroxyl groups have a dual effect of increasing inhibitory activity while preventing cytotoxicity.

The inhibitory activity observed in vitro was also observed in vivo with selected sesquiterpenes inhibiting neutrophil superoxide production. These compounds also had the unexpected effect of inhibiting neutrophil infiltration. Although it is unknown why the inhibition of neutrophil superoxide production may also affect neutrophil infiltration, the compounds were also shown to inhibit superoxide production on vascular cells: a process that is involved in neutrophil recruitment. Hence it is possible that inhibition of superoxide production by vascular cells may play a role in the inhibition of neutrophil infiltration.

It is also possible that the sesquiterpenes may be having an effect on neutrophil recruitment through influencing macrophage activation. Although a clear pattern relating chemical structure to the effects on RAW 264.7 cytokine production could not be established, nevertheless the compounds were able to affect the production of IL-6 and TNF $\alpha$ from MSU-stimulated RAW 264.7 cells further indicating that there are secondary effects of polygodial and the sesquiterpene dialadehydes on cells other than neutrophils. 


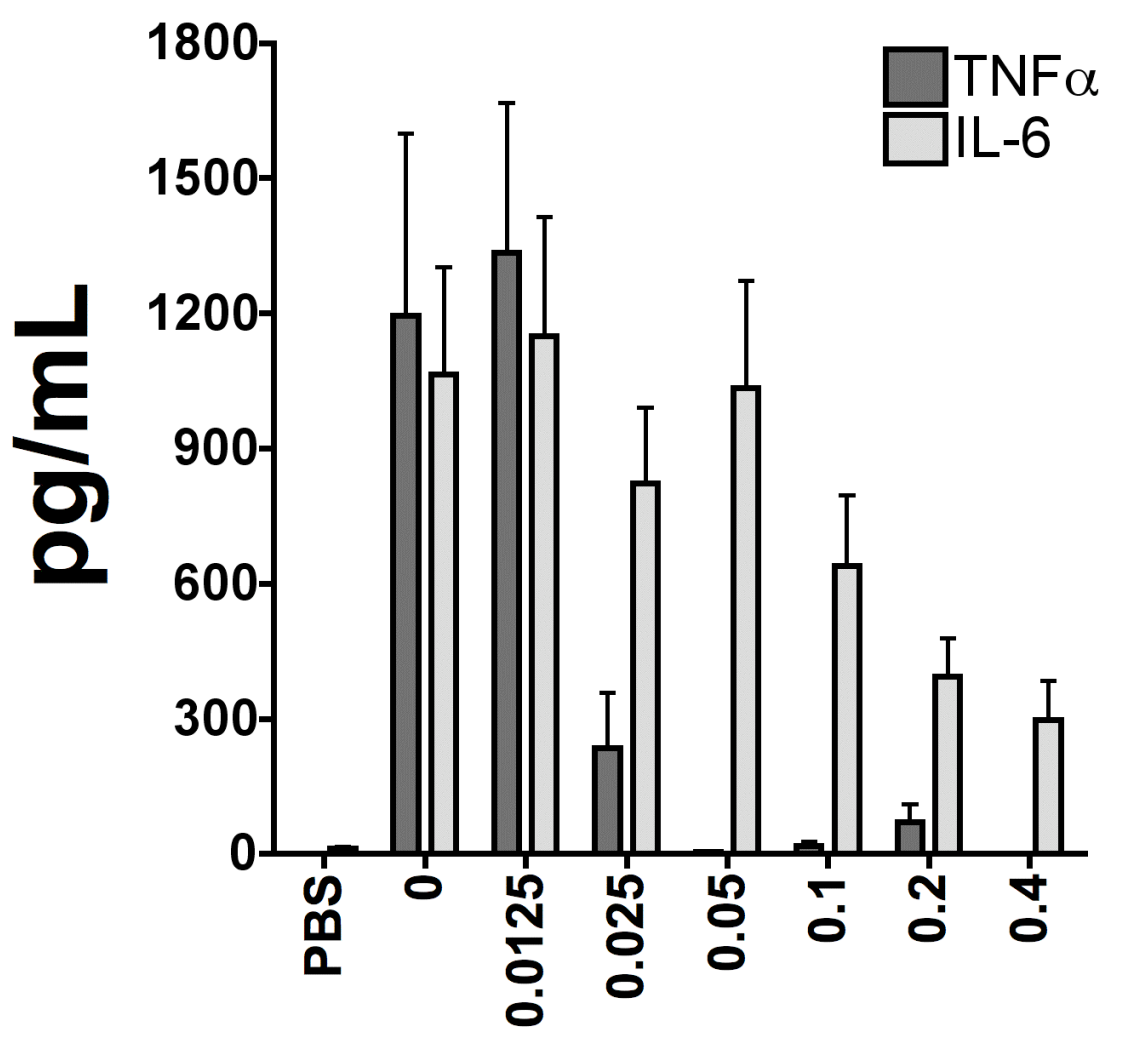

$\%$ DMSO (+200 $\mu \mathrm{g} / \mathrm{mL}$ MSU)

Figure 4.7 The effect of DMSO on MSU-stimulated cytokine production. RAW 264.7 cells were cultured with $200 \mu \mathrm{g} / \mathrm{mL}$ MSU in vitro for $24 \mathrm{~h}$ in the presence of different concentrations of DMSO. The production of TNF $\alpha$ and IL-6 were measured in the supernatants by ELISA. Measurements were performed in triplicate and values represent mean + S.E.M. Results are representative of three separate experiments. 
A
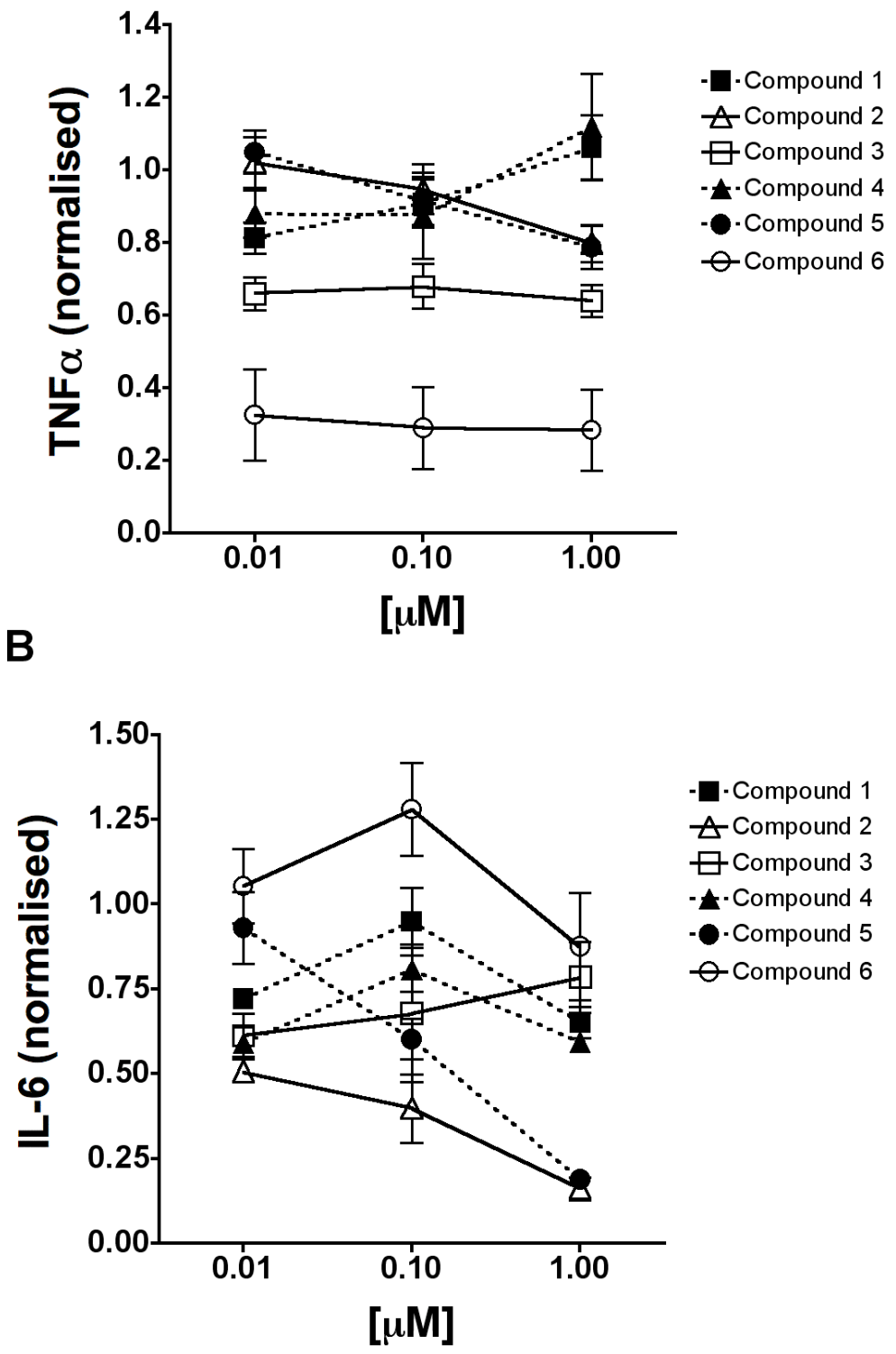

Figure 4.8 The effect of sesquiterpene compounds on MSU-stimulated cytokine production. RAW 264.7 cells were cultured with $200 \mu \mathrm{g} / \mathrm{mL}$ MSU in vitro for $24 \mathrm{~h}$ in the presence of different concentrations of sesquiterpene compounds. The production of (A) TNF $\alpha$ and (B) IL-6 were measured in the supernatants by ELISA. Values within each experiment were normalised to the concentration of TNF $\alpha$ or IL6 produced in the presence of MSU and no sesquiterpene compound. Values for three separate experiments were combined to give the above results. Measurement in each experiment were performed in triplicate and values above represent mean \pm S.E.M. 
The anti-inflammatory effect of sesquiterpene dialdehydes on both neutrophil infiltration and neutrophil activation identifies the clear potential for further study of these compounds as promising candidates for development as anti-inflammatory drugs for the treatment neutrophil-driven disease. Indeed, these results clearly indicate that these compounds are able to inhibit MSU-induced neutrophilia, immediately identifying acute gout as a possible disease target for the therapeutic development of these compounds.

The wide range of inflammatory effects of sesquiterpene dialdehydes leads to the question of how these compounds may be exerting their effect on a molecular level. Unfortunately, there is little information in the literature to give a clear indication of the mode of action of these compounds as anti-inflammatories. However, there does appear to be an indication that dialdehydes such as polygodial bind to thiol groups on proteins (Jansen and de Groot, 2004). If this is the case, then it is entirely possible that sesquiterpene dialdehydes accomplish a wide range of activities by reacting with more than one thiol-containing protein or enzyme. Whether proteins other than NADPH oxidase are additional targets for these compounds is a subject for future research.

In summary, polygodial and the other sesquiterpene dialdehyde compounds showed a number of anti-inflammatory activities including the inhibition of neutrophil superoxide production that translated into an in vivo model of gout. The activity of polygodial could be improved as shown by Compounds 2 and 4, making them favourable candidates for drug development for use in gout and other neutrophil based inflammatory diseases. 
Chapter 5

Macrophages in the initiation of acute gout 


\section{Chapter 5}

\section{Macrophages in the initiation of acute gout}

\subsection{Introduction}

Early inflammation in acute gout is associated with the production of proinflammatory cytokines (IL-6, TNF $\alpha$, IL-1 $\beta$ ) and a neutrophil-rich cellular infiltration (Di Giovine et al., 1987, Guerne et al., 1989, di Giovine et al., 1991, Schiltz et al., 2002). A number of cell types have been proposed to be orchestrators in the inflammatory events that surround gouty attacks; including monocytes, mast cells, and epithelial cells.

Previous in vitro and ex vivo studies have implicated monocytes as key cells involved in the inflammatory phase of gouty inflammation, whilst claiming macrophages as raising anti-inflammatory responses to MSU stimulation. The results of these studies were based on immortalised cell lines expressing monocytelike and macrophage-like phenotypes that were exposed to MSU in vitro. Monocytes were shown to produce pro-inflammatory cytokines such as TNF $\alpha$, IL-6 in response to MSU crystals whereas macrophage cultures failed to produce TNF $\alpha$ and IL-6, indicating a possible non-inflammatory response to MSU that could be associated with resolution of inflammation (Yagnik et al., 2000).

A similar pattern of monocyte pro-inflammatory responses and macrophage antiinflammatory responses has also been reported in studies using human CD14 monocytes that had been purified from peripheral blood and then differentiated into macrophages in vitro. Freshly isolated monocytes produced TNF $\alpha$ and IL-1 $\beta$ in response to MSU stimulation in vitro; whilst monocytes that had been differentiated into macrophages and then exposed to MSU 'switched' from producing TNF $\alpha$ and IL-1 $\beta$, to producing TGF $\beta$, an 'anti-inflammatory' cytokine commonly linked to resolution of inflammation (Yagnik et al., 2004). Isolated cellular infiltrates from catharidin-induced blisters in humans have also shown a switch to TGF $\beta$ production 
following restimulation of 16 hour and 48 hour infiltrates with MSU ex vivo (Yagnik et al., 2004).

Together, these results have led to the hypothesis that, in an acute gout attack, monocytes drive the inflammatory phase of the response while differentiated macrophages mediate its resolution. However there are limitations associated with these studies due to the artificial nature of the in vitro models used. In addition, despite indications of switching to TGF $\beta$ production in human inflammation the source of cytokine production was not identified and the monocyte/macrophage phenotypes were not characterised. Therefore, more thorough studies are required to establish which monocyte/macrophage phenotypes are specifically responsible for cytokine production in the context of MSU-induced inflammation in vivo.

In addition to the limitations associated with the studies described above, more recent literature now indicates a possible pro-inflammatory role for macrophages in gouty inflammation. Macrophages treated with MSU have been reported to induce pro-inflammatory cytokines such as IL-1 $\beta$ where signalling through the IL-1 receptor is required to activate non-hemopoietic cells to induce neutrophil infiltration (Chen et al., 2006, Martinon et al., 2006), a key component in gouty inflammation (Phelps and McCarty, 1966). Other pro-inflammatory activities such as the increase of TNF $\alpha$, MCP-1, IL-18, iNOS and the upregulation of TREM-1 on macrophages points to a potential involvement of this cell type in gout (Jaramillo et al., 2004b, Jaramillo et al., 2004a, Chen et al., 2006, Martinon et al., 2006, Murakami et al., 2006). This would be consistent with other acute inflammatory conditions that rely heavily on macrophages in the onset of inflammation (Knudsen et al., 2002, Cailhier et al., 2005).

Although both macrophages and monocytes appear to produce pro-inflammatory cytokines (IL-1 $\beta$, IL-6, TNF $\alpha$ ) following in vitro stimulation with MSU, these activities have not been confirmed in an in vivo model of gout. This would be an important step towards understanding the behaviour of these cells in vivo as the local inflammatory environment heavily influences the functional phenotype of monocytes and macrophages (Stout and Suttles, 2004). Hence, the in vitro 
experiments based on the stimulation of murine cell lines and differentiating CD14 human monocytes may not adequately simulate the complex in vivo conditions that are likely to influence both the development and the response of these cells during a gouty inflammatory response. In addition, blister exudates recruited in response to an irritant that is physiologically irrelevant to gout may represent inflammation and cell profiles that have very little to do with those induced by MSU crystals.

These considerations identify a requirement for further investigation into the role of mononuclear phagocytes in initiation and progression of gouty inflammation in vivo. In vivo studies using MSU as the inducing factor, and distinguishing between the different cell types and various phenotypes of mononuclear phagocyte populations over the course of the inflammatory response would provide a more accurate depiction of the function of these cells in the context of gouty arthritis.

This chapter therefore addresses these issues using a murine peritoneal model of acute gout. Using this model I sought to identify the responses of monocytes and macrophages during an inflammatory response to MSU, specifically the contribution these cells make to the early phase of gouty inflammation.

\subsection{Results}

\subsubsection{Characterising the murine peritoneal model of acute gout.}

Since no study had previously examined the role of monocytes and macrophages in gout in vivo, a combination of surface markers was sought to clearly distinguish monocytes from macrophages during MSU-induced inflammation.

To identify different cell populations, peritoneal leukocytes were harvested from mice that had been treated with MSU for four hours and were stained for the macrophage differentiation marker F4/80 and the myeloid differentiation antigens Gr-1 and clone 7/4 antigen (Fig. 5.2). Monocytes and neutrophils both expressed Gr-1 and 7/4, but were distinguishable by their different levels of expression. Neutrophils were identified as $7 / 4^{+}, \mathrm{Gr}-1^{\text {hi }}$ (Fig. 5.1A, gate R1) and monocytes as $7 / 4^{+}$, Gr- $1^{\text {int }}$ (Fig. 5.1A, gate R2) expressing low levels of F4/80 (Fig. 5.1B, gate R3). 

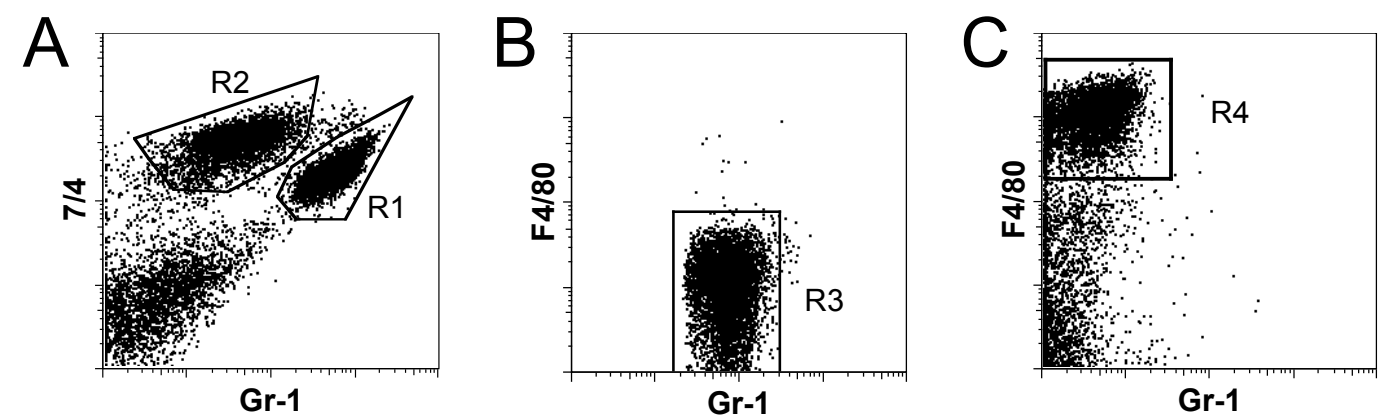

Figure 5.1 Identification of cell types involved in the MSU crystal response in vivo. Mice were treated with MSU crystals i.p. (3mg, $0.5 \mathrm{~mL}$ PBS). After 4 hours peritoneal cells were harvested by lavage $(3 \mathrm{~mL}, \mathrm{PBS})$ and the cell types identified by flow cytometry. (A) Neutrophils were identified as Gr-1 ${ }^{\text {hi }}, 7 / 4^{+}$(Gate R1) while monocytes were Gr-1 ${ }^{\text {int }}, 7 / 4^{+}(\mathbf{A})\left(\right.$ Gate R2) and F4/80 $0^{\text {lo }}$ (Gate R3)(B). (C) F4/80 hi resident macrophages (Gate R4) were identified in untreated mice. Results are representative of three separate experiments. 
Given that the mouse peritoneum in C57/BL6 mice is known to consist of 50-80\% resident peritoneal macrophages, peritoneal cells were harvested from naïve mice and stained with the same markers to distinguish macrophages in this model. Resident macrophages had quite a distinctive staining, expressing much higher levels of F4/80 than monocytes while lacking expression of both Gr-1 and 7/4 (Fig. 5.1C, gate R4).

To establish conclusively that the staining protocol was identifying the correct cell types, the above populations were sorted and the different cell types observed morphologically. Cells that were $7 / 4^{+}, \mathrm{Gr}-1^{\text {hi }}$ were confirmed as neutrophils, having nuclei with a "string" like appearance typical of polymorphonuclear cells (Fig. 5.2A); while $7 / 4^{+} / \mathrm{Gr}-1^{\text {int }}$ cells were monocytes, containing a more "bean" shaped nucleus (Fig. 5.2B). Interestingly, monocytes isolated during the early response were quite morphologically distinct from the sorted $\mathrm{F} 4 / 80^{\mathrm{hi}}$ resident macrophages, which had a larger cytoplasm and more rounded nuclei (Fig. 5.2C). These morphological observations confirmed the correct identification of the appropriate cell types of interest using expression of F4/80, Gr-1 and 7/4.

Using this method of distinguishing the cell types of interest, leukocyte infiltration and the production of pro-inflammatory cytokines (IL-1 $\beta$, IL-6, MCP-1 and TNF $\alpha$ ) was followed over 72 hours in order to establish the acute inflammatory profile of peritoneal inflammation in response to MSU. MSU induced a large cellular infiltration that peaked at $16 \mathrm{~h}$ after MSU administration (Fig. 5.3A). Cellular infiltrate was largely comprised of monocytes and neutrophils, both of which peaked at 16 hours after MSU administration (Fig. 5.3B). Neutrophil infiltration was notably faster and larger in number than that of monocytes. Cytokine analysis of the PBS lavage fluid revealed that IL-6, IL-1 $\beta$ and TNF $\alpha$ levels were elevated in the peritoneum of MSU-treated mice within 2 hours of MSU administration, peaking at four hours for all four cytokines (Fig. 5.3C). The inflammatory response was selflimiting with leukocyte cell numbers and cytokine levels returning to normal within 48 hours. (For a more comprehensive breakdown of cell types in the peritoneum during the response to MSU, see Table 5.1). 

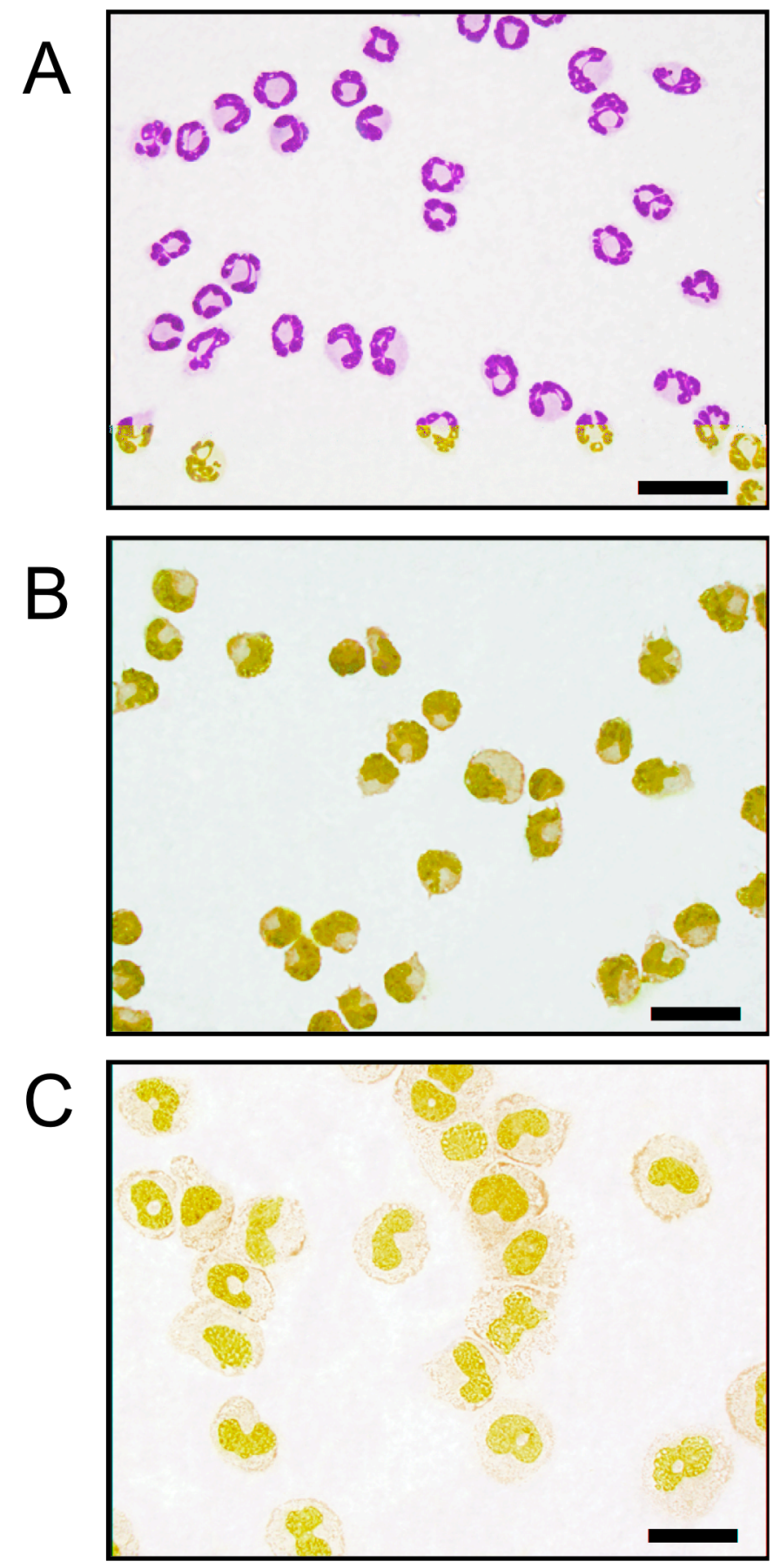

Figure 5.2 Morphology of cells expressing the myeloid antigens 7/4 and Gr-1.

Mice were treated with MSU crystals i.p. and after 8 hours peritoneal cells were harvested by lavage. Cells were then surface stained for Gr-1 and 7/4 and sorted based on levels of expression. (A) Gr- $1^{\mathrm{hi}}, 7 / 4^{+}$cells were neutrophils while (B) Gr$1^{\text {int }}, 7 / 4^{+}$cells had the morphology of monocytes. (C) $\mathrm{F} 4 / 80^{\mathrm{hi}}, \mathrm{Gr}-1^{-}, 7 / 4^{-}$resident macrophages were identified in untreated mice. Original magnification 100x. Scale bar represents $20 \mu \mathrm{m}$. Results are representative of three separate experiments. 
Figure 5.3 MSU induces cellular infiltration and cytokine production in vivo. Mice were treated with MSU i.p. (3mg; $0.5 \mathrm{~mL}$ PBS) and at various timepoints mice were sacrificed. Cells in the peritoneal cavity were harvested by lavage (3mL PBS). (A) Monocytes and neutrophils were identified by FACS using Gr-1 and 7/4. (B) Monocyte and neutrophil numbers were determined by FACS over 72 hours. (C) Supernatants from the peritoneal lavages were analysed for cytokines using CBA. Values represent mean \pm S.E.M. $(n=3$ mice per group). Results are representative of three separate experiments. 
A

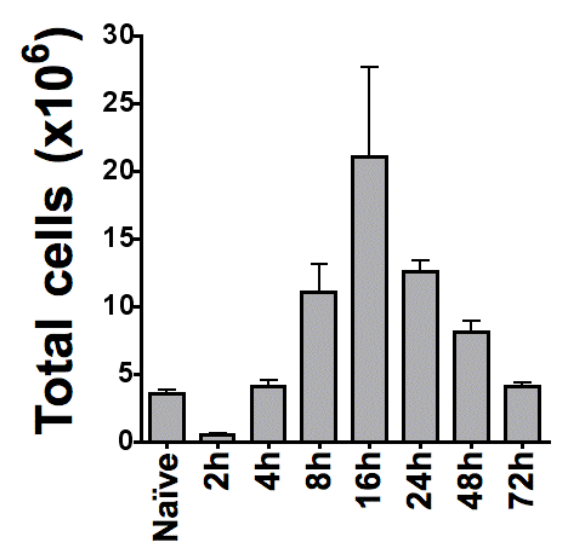

C
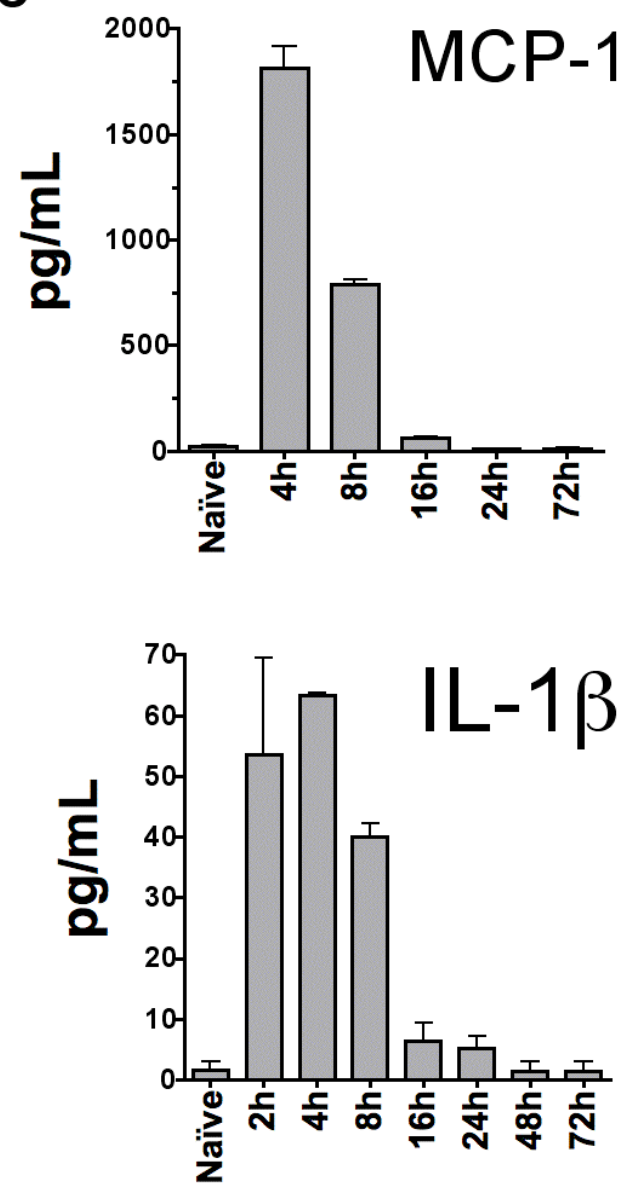

B
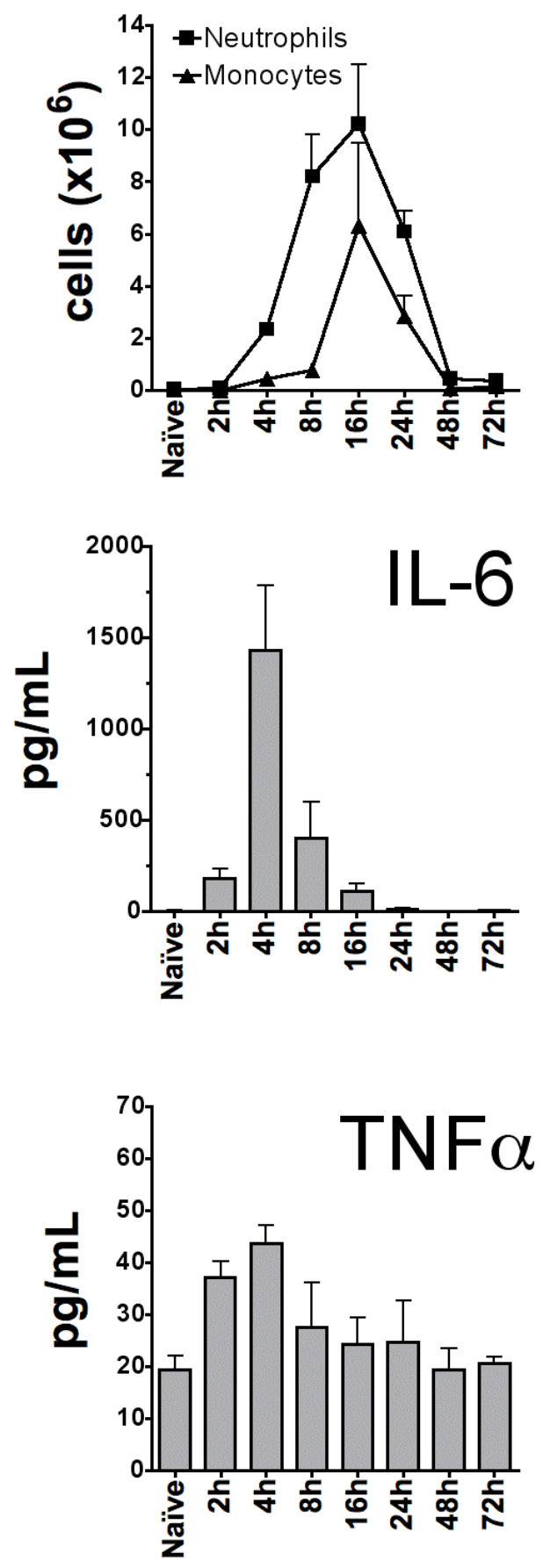


\begin{tabular}{|c|c|c|c|c|}
\cline { 2 - 5 } A & $\begin{array}{c}\text { Macrophage/ } \\
\text { Monocytes }\end{array}$ & Neutrophils & Lymphocytes & Mast cells \\
\hline Naïve & $83.9 \pm 1.4$ & $0.0 \pm 0.0$ & $14.1 \pm 1.2$ & $2.7 \pm 0.7$ \\
4h & $23.8 \pm 1.3$ & $67.4 \pm 5.2$ & $8.4 \pm 3.9$ & $0.0 \pm 0.0$ \\
8h & $30.4 \pm 2.4$ & $67.7 \pm 2.2$ & $1.2 \pm 0.3$ & $0.0 \pm 0.0$ \\
16h & $40.5 \pm 4.1$ & $56.4 \pm 3.0$ & $1.6 \pm 0.1$ & $0.2 \pm 0.2$ \\
24h & $85.9 \pm 3.3$ & $8.3 \pm 1.7$ & $3.1 \pm 1.0$ & $0.0 \pm 0.0$ \\
72h & $88.3 \pm 0.9$ & $3.6 \pm 0.9$ & $5.8 \pm 1.7$ & $0.2 \pm 0.1$ \\
\hline
\end{tabular}

\begin{tabular}{|c|c|c|c|c|}
\hline & $\begin{array}{c}\text { Macrophage/ } \\
\text { Monocytes }\left(\times 10^{6}\right)\end{array}$ & $\begin{array}{c}\text { Neutrophils } \\
\left(\times 10^{6}\right)\end{array}$ & $\begin{array}{c}\text { Lymphocytes } \\
\left(\times 10^{5}\right)\end{array}$ & Mast cells \\
\hline $\begin{array}{l}\text { Naïve } \\
4 \mathrm{~h} \\
8 \mathrm{~h} \\
16 \mathrm{~h} \\
24 \mathrm{~h} \\
72 \mathrm{~h}\end{array}$ & $\begin{array}{l}1.7 \pm 0.4 \\
0.7 \pm 0.3 \\
2.7 \pm 0.6 \\
4.6 \pm 1.4 \\
4.9 \pm 1.5 \\
3.3 \pm 1.2\end{array}$ & $\begin{array}{l}0.0 \pm 0.0 \\
2.4 \pm 0.7 \\
8.9 \pm 1.9 \\
8.6 \pm 1.1 \\
2.0 \pm 1.4 \\
0.2 \pm 0.1\end{array}$ & $\begin{array}{l}4.3 \pm 0.8 \\
2.3 \pm 0.6 \\
1.6 \pm 0.7 \\
2.4 \pm 0.3 \\
2.7 \pm 0.9 \\
2.6 \pm 0.5\end{array}$ & $<6 \times 10^{5}$ \\
\hline
\end{tabular}

Table 5.1 Cell composition of the peritoneum during an inflammatory response to MSU crystals. Mice were treated with MSU i.p. (3mg; 0.5mL PBS) as in Fig. 5.3, and at various timepoints mice were sacrificed. Cells in the peritoneal cavity were harvested by lavage ( $3 \mathrm{~mL}$ PBS). A. The percentage composition of the cells in the peritoneum at various times after MSU treatment. B. The absolute numbers of cells in the peritoneum at various times after MSU treatment. Values represent a combination of three separate experiments and show mean \pm S.E.M. 
Based on peak cytokine production combined with early leukocyte infiltration I chose to focus on the first 4 to 8 hours to determine which cell types, monocytes or macrophages, were driving the early inflammatory response to MSU.

\subsubsection{Production of pro-inflammatory cytokines by cells stimulated with MSU crystals}

Monocytes have been reported to play a pivotal role in the inflammatory response to MSU. To investigate the contribution of infiltrating monocytes to early inflammation following MSU exposure, peritoneal exudate cells were collected from mice four hours after treatment with MSU and examined for the production of the proinflammatory cytokines IL- 6 and TNF $\alpha$ by infiltrating $7 / 4^{+} / \mathrm{Gr}-1^{\text {int }}$ monocytes. Despite high levels of IL-6 being present in the peritoneum at 4 to $8 \mathrm{~h}$, the infiltrating monocyte population was not producing IL-6 although they were still able to produce IL-6 in response to LPS stimulation in vitro (Fig. 5.4, upper panels). In fact, none of the harvested cells appeared to be producing large amounts of IL-6 (Fig. 5.4, middle and lower panels). This indicated that although monocytes had the potential to produce IL-6 in vitro, they were not producing IL-6 when entering the peritoneum in vivo.

Although monocytes did not appear to be producing IL-6, they did, however, stain positively for TNF $\alpha$ with $11 \%$ of monocytes being $\mathrm{TNF}^{+}$(Fig. 5.5, upper panel). Although this implicated monocytes as a source of $\mathrm{TNF} \alpha$, positive staining was not a feature unique to monocytes, but was also present in a small percentage $(<4 \%)$ of neutrophils and the remaining cells in the peritoneal wash. It is possible that this general TNF $\alpha$ staining of cells was the result of the anti-TNF $\alpha$ antibodies binding to intracellular stores of TNF $\alpha$ rather than MSU-induced TNF $\alpha$ per se. In order to determine whether this positive staining represented inducible TNF $\alpha$, these cells were exposed to MSU ex vivo and the levels of cytokines actually released from the recruited cells assayed. As a comparison, cells from the peritonea of naïve mice were also tested. Cells from naïve peritonea contain mainly macrophages, in contrast to cells from MSU-treated peritonea which contain monocytes and neutrophils. 


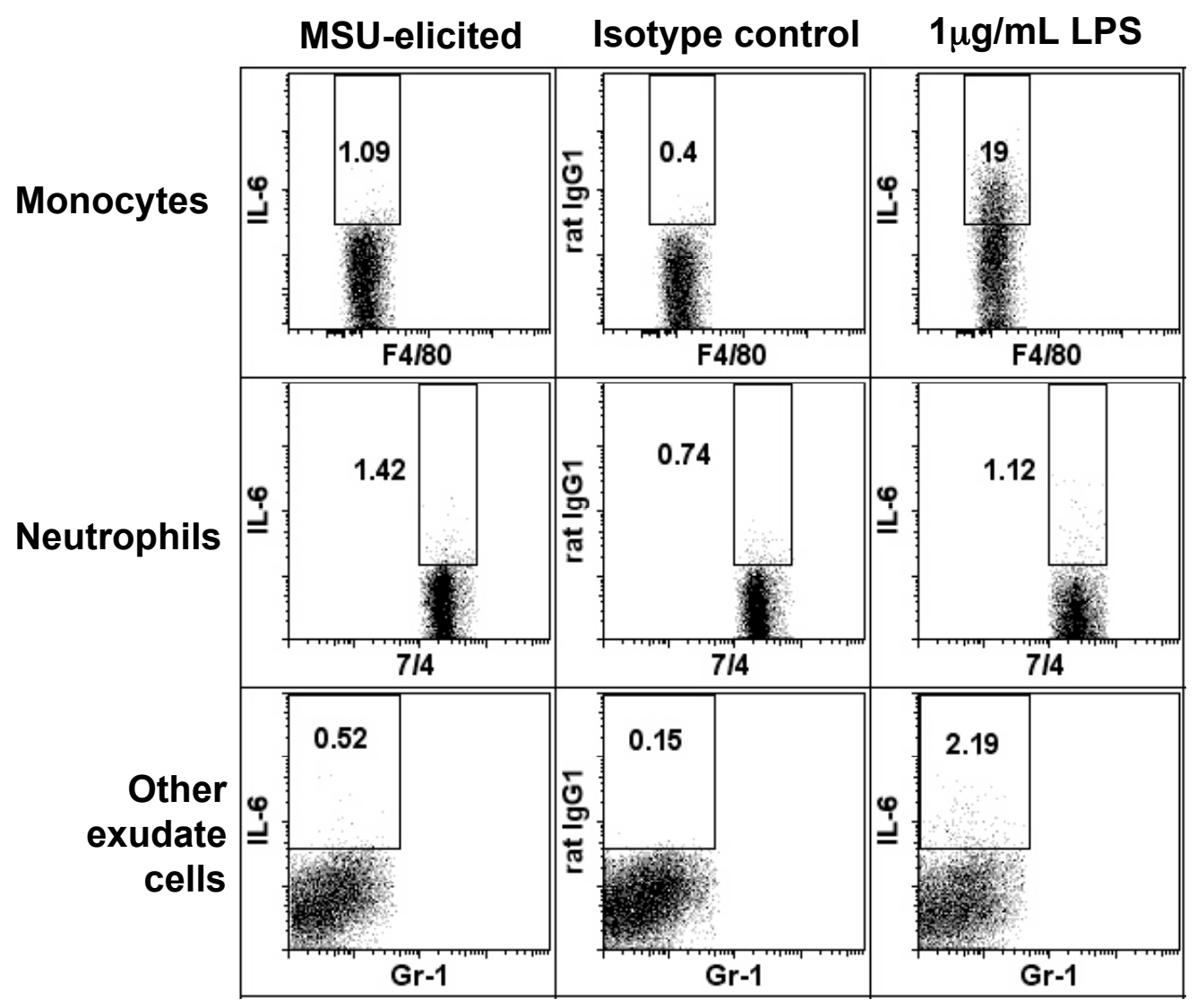

Figure 5.4 IL-6 production by cells infiltrating the peritoneum in response to MSU. Mice were treated with MSU i.p. (3mg; $0.5 \mathrm{~mL}$ PBS) and after 4 hours the cells in the peritoneal cavity were harvested by lavage ( $3 \mathrm{~mL}$ PBS). Peritoneal cells were analysed by FACS to identify IL-6 production by monocytes, neutrophils or the remaining leukocytes in the lavage. Results are representative of three separate experiments. 


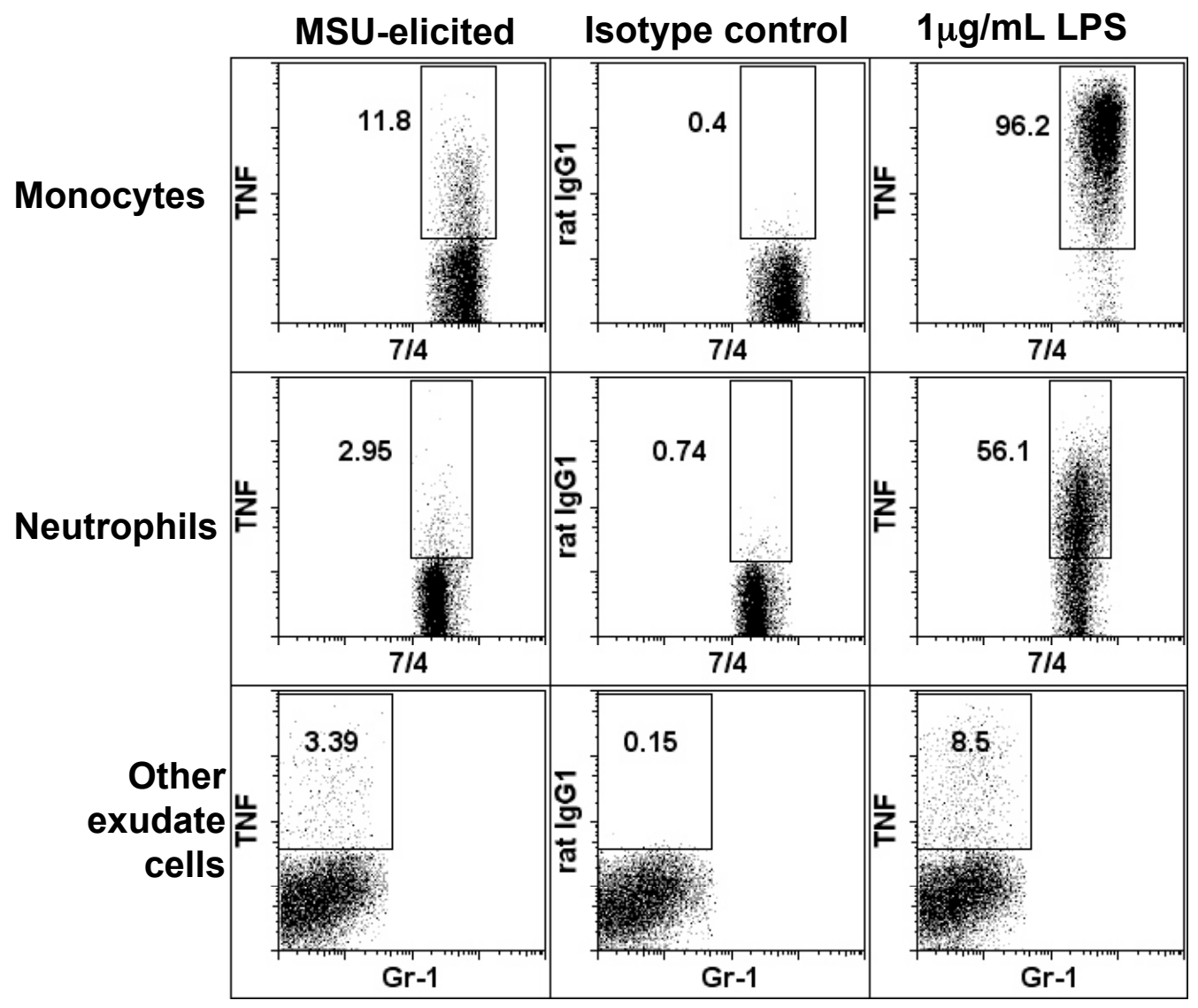

Figure 5.5 TNF $\alpha$ production by cells infiltrating the peritoneum in response to MSU. Mice were treated with MSU i.p. (3mg; 0.5mL PBS) and after 4 hours the cells in the peritoneal cavity were harvested by lavage (3mL PBS). Peritoneal cells were analysed by FACS to identify TNF $\alpha$ production by monocytes, neutrophils or the remaining leukocytes in the lavage. Results are representative of three separate experiments. 
Cells from MSU-treated mice could not be stimulated to release TNF $\alpha$ following exposure to MSU; nor did they produce IL-1 $\beta$, IL-6 or MCP-1 (Fig. 5.6). Although these cells produced background levels of each of these cytokines, these levels were no greater than the background levels observed from cells of naïve mice, which had received no inflammatory stimulation in vivo (Fig. 5.6). In contrast to recruited monocytes (and recruited cells in general at $4 \mathrm{~h}$ ) resident cells from naïve mice responded to MSU exposure, producing high levels of cytokines following exposure and revealing macrophages to be more pro-inflammatory than recruited monocytes.

These results revealed two unexpected features of monocytes newly recruited by MSU: they do not secrete the cytokines IL-6, TNF $\alpha$, IL-6 and MCP-1, and more surprisingly, they cannot be stimulated by MSU to produce these cytokines (Fig. 5.6). This led me to ask the question of whether this phenomenon was distinct to monocytes recruited by MSU or whether this was a general feature of inflammatory monocytes. To determine this, an alternative inflammatory stimulus was used to induce leukocyte recruitment to the peritoneum.

An i.p. injection of $4 \%$ thioglycollate broth induced the recruitment of both monocytes and neutrophils as soon as $4 \mathrm{~h}$ after administration (Fig. 5.7A). Similar to MSU-recruited exudate cells, thioglycollate-recruited cells were not responsive to MSU in vitro (Fig. 5.7B). These results indicated that non-responsiveness of newly recruited monocytes to MSU is likely to be a general characteristic of elicited monocytes regardless of the stimulus used to induce recruitment.

\subsubsection{Investigating cytokine responses of purified monocytes and neutrophils to MSU stimulation}

To confirm that monocytes were unable to produce cytokines (IL-1 $\beta$, TNF $\alpha$, IL-6, $\mathrm{MCP}-1$ ) in response to MSU, monocytes were isolated from mice $8 \mathrm{~h}$ after MSU treatment by FACS and restimulated with MSU ex vivo. The sorting procedure caused a high non-specific background cytokine production from monocytes; nevertheless, purified monocytes showed no additional response to MSU indicating that monocytes were once again an unlikely in vivo source of these cytokines (Fig. $5.8 \mathrm{~A})$. 

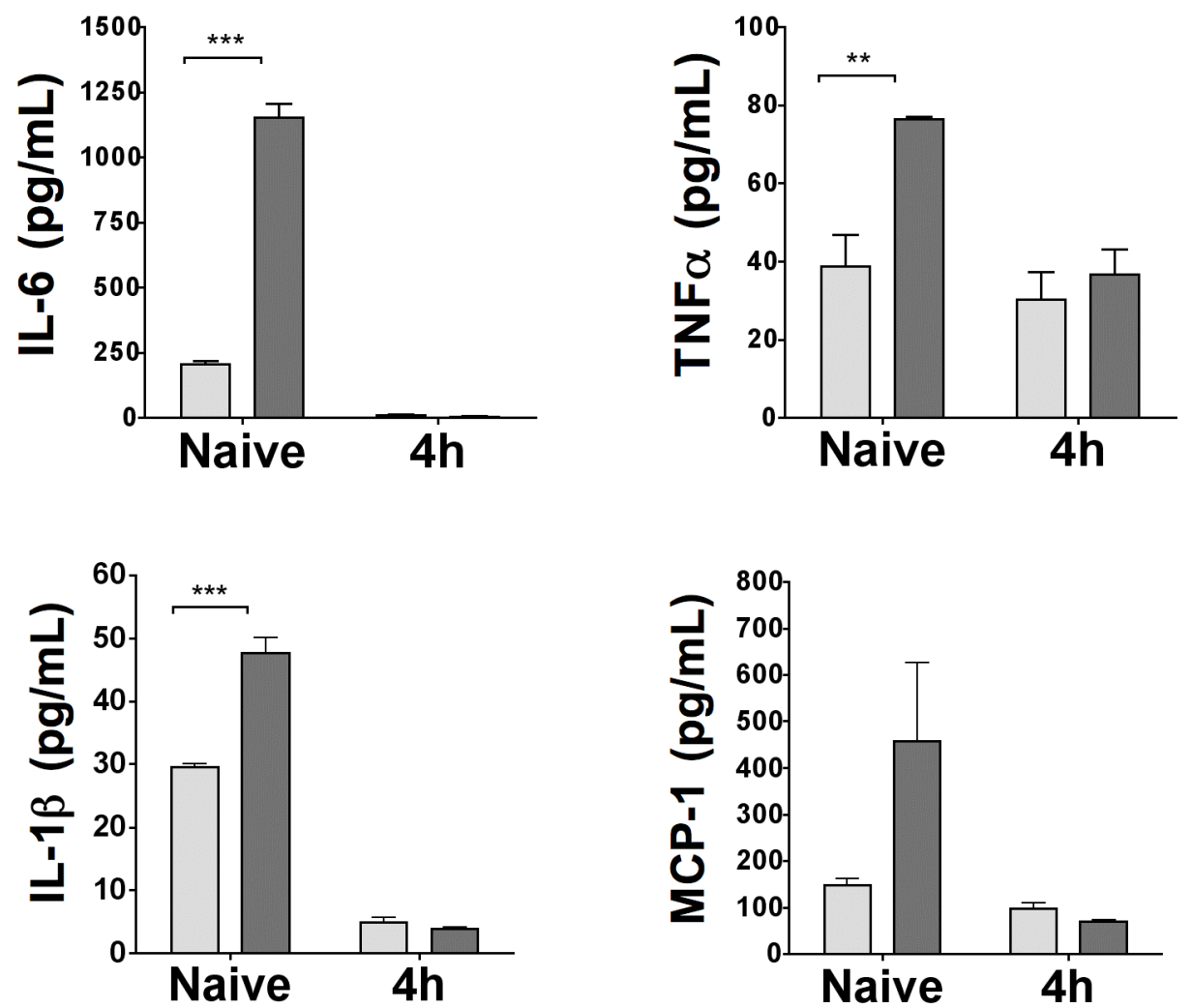

Figure 5.6 Infiltrating leukocytes are unresponsive to MSU. Peritoneal cells were isolated from mice 4 hours after administration of MSU i.p. or naïve mice and were restimulated with $200 \mu \mathrm{g} / \mathrm{mL}$ MSU ex vivo for $16 \mathrm{~h}$. Supernatants from cell cultures were analysed for IL-6, TNF $\alpha$, IL-1 $\beta$ and MCP-1 using ELISA and CBA. Dark bars represent cells restimulated with MSU crystals, while light bars represent PBS controls. Measurements were performed in quadruplicate. Values represent mean \pm S.E.M. ${ }^{* *}=P<0.01$ and $* * *=P<0.001$ as determined by Student's $t$-test against the indicated control. Results are representative of three separate experiments. 
A

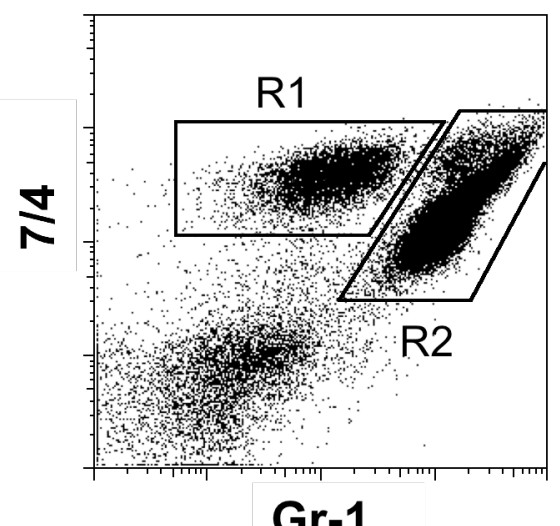

B

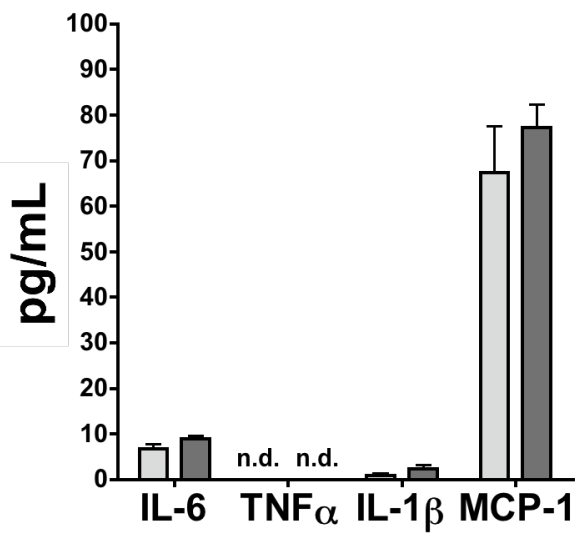

Figure 5.7 Leukocytes infiltrating in response to thioglycollate are unresponsive to MSU. Mice were treated with an i.p. injection of thioglycollate $(4 \%, 0.5 \mathrm{~mL})$ and after six hours peritoneal cells were isolated from mice by peritoneal lavage $(3 \mathrm{~mL}$ PBS). A, Peritoneal exudates cells contained both monocytes (R1) and neutrophils (R2). B, Harvested peritoneal leukocytes were restimulated with $200 \mu \mathrm{g} / \mathrm{mL} \mathrm{MSU}$ ex vivo and after $16 \mathrm{~h}$ the supernatants from cell cultures were analysed for cytokines using ELISA and CBA. Dark bars represent MSU-treated cells while light bars represent PBS controls. Measurements were performed in triplicate. Values represent mean \pm S.E.M. The experiment in Figure 5.7B was performed by $\mathrm{M}$. Walton. 
Because neutrophils were also present in high numbers in the mixed cellular exudate, purified recruited neutrophils were exposed to MSU in vitro and tested as a possible source of IL-1 $\beta$, TNF $\alpha$, IL-6, or MCP-1. Despite a high non-specific background production of MCP-1, neutrophils did not produce pro-inflammatory cytokines in response to MSU, ruling them out as a potential source of the cytokines observed in vivo (Fig. 5.8B).

Collectively these data provided strong evidence for the idea that neither monocytes nor neutrophils are significant contributors to the generation of the pro-inflammatory cytokines (TNF $\alpha$, IL-1 $\beta$, IL-6 or MCP-1) in MSU-induced inflammation.

\subsubsection{Identifying cells that produce pro-inflammatory cytokines after exposure to MSU crystals}

The discovery that monocytes were not capable of producing TNF $\alpha$, IL-1 $\beta$, IL-6 and MCP-1 during the inflammatory phase of gout in vivo led to the question of whether cells present locally at the time that inflammation was initiated would be involved in driving inflammation. I had previously noted that peritoneal leukocyte cells from naïve mice produced significant amounts of inflammatory cytokines following exposure to MSU ex vivo, implicating macrophages as a possible source of proinflammatory cytokines in vivo (Fig. 5.6).

Resident peritoneal leukocytes are a mixture of cells primarily composed of macrophages, and therefore it seemed reasonable to assume that macrophages were the source of IL- 6 , IL-1 $\beta$ and TNF $\alpha$ observed in stimulated peritoneal leukocyte cultures above. To confirm that macrophages were they key source of these cytokines in the peritoneal leukocytes from naïve mice, different resident cell populations were separated into macrophages, lymphocytes and mast cells and then exposed to MSU in vitro for $8 \mathrm{~h}$. Although lymphocyte and mast cell populations were able to produce low levels of IL-6, IL- $1 \beta$ and TNF $\alpha$, macrophages produced a much higher level of all three cytokines following MSU exposure (Fig. 5.9). The results identified macrophages as the primary source of IL-6, IL-1 $\beta$ and TNF $\alpha$ in response to MSU in the peritoneal leukocyte population. 


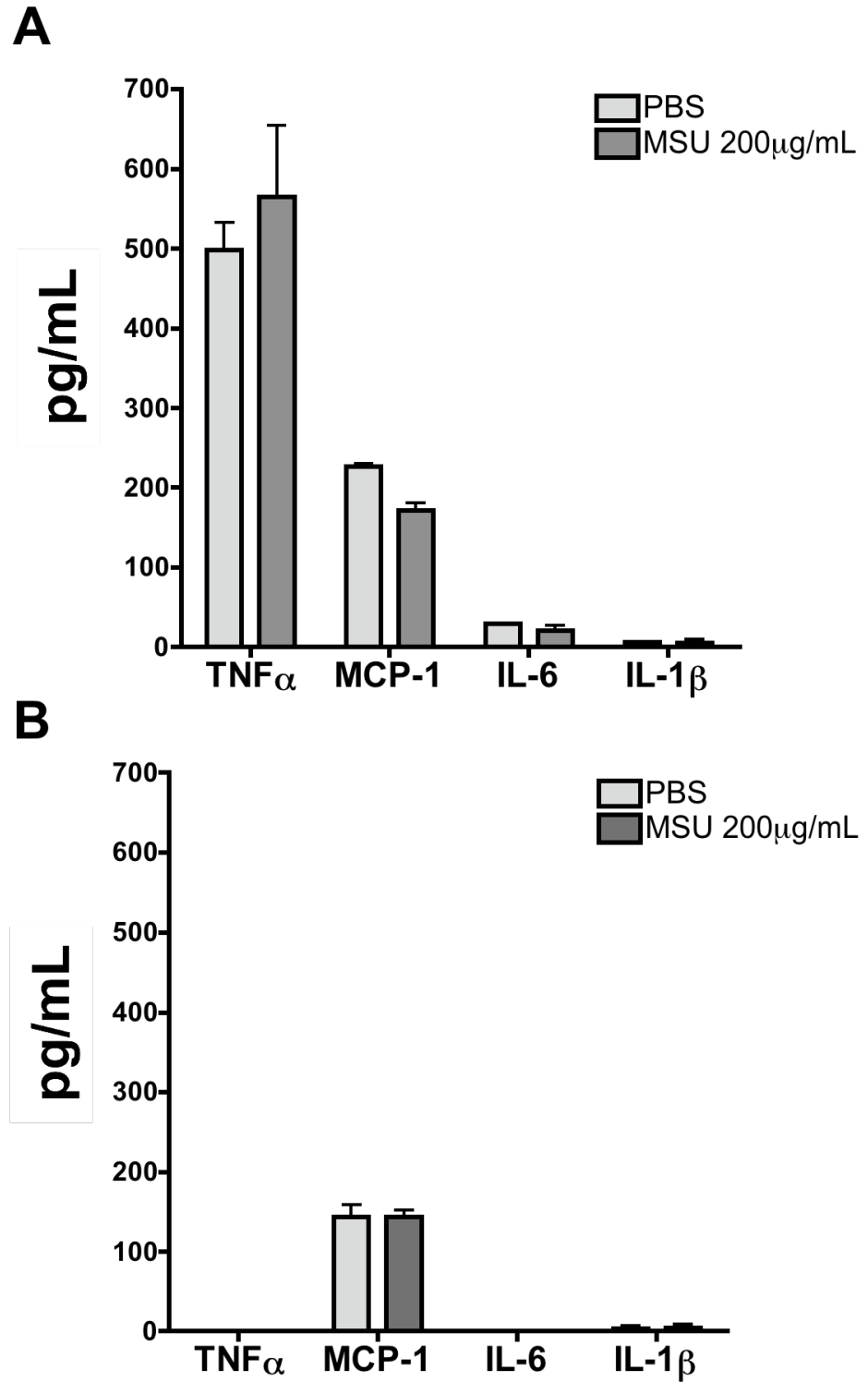

Figure 5.8 MSU-elicited monocytes and neutrophils are unresponsive to MSU. Mice were treated with an i.p. injection of MSU (3mg, $0.5 \mathrm{~mL}$ PBS) and eight hours later peritoneal cells were isolated by lavage $(3 \mathrm{~mL} \mathrm{PBS}) . \mathrm{F} 4 / 80^{\mathrm{lo}}, \mathrm{Gr}-1^{\mathrm{int}}, 7 / 4^{+}$ monocytes and neutrophils $\mathrm{F} 4 / 80^{-}, \mathrm{Gr}-1^{-}, 7 / 4^{+}$were purified by FACS and restimulated with $200 \mu \mathrm{g} / \mathrm{mL}$ MSU ex vivo. After $24 \mathrm{~h}$, supernatants were analysed for cytokines by ELISA and CBA. A. Cytokine production from purified monocytes. B. Cytokine production from purified neutrophils. Dark bars represent MSU treated monocytes while light bars represent PBS controls. Measurements were performed in triplicate. Values represent mean \pm S.E.M. Results are representative of three separate experiments. 
A

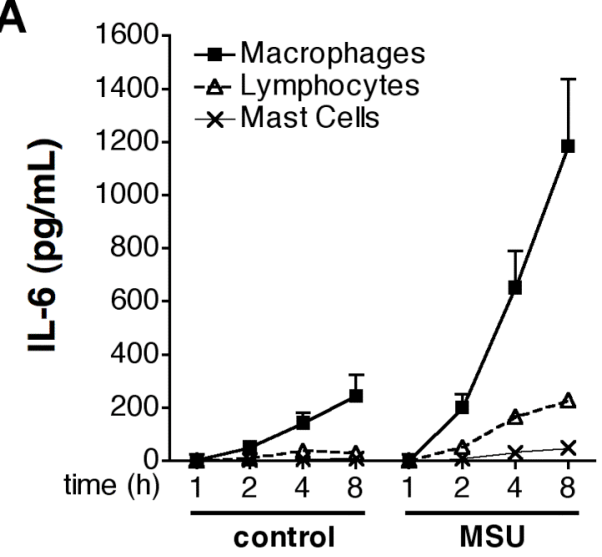

B

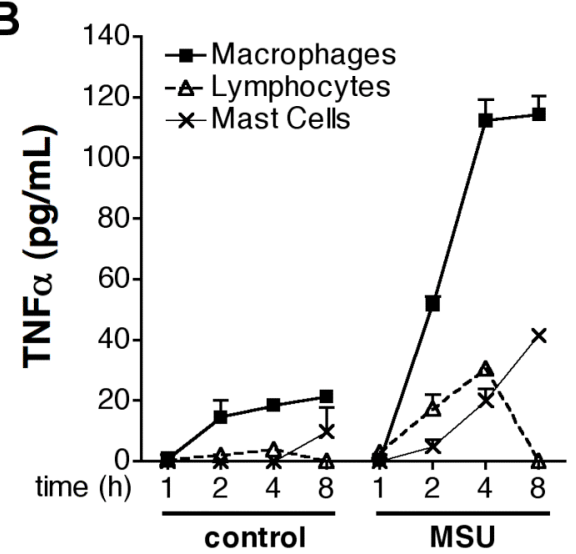

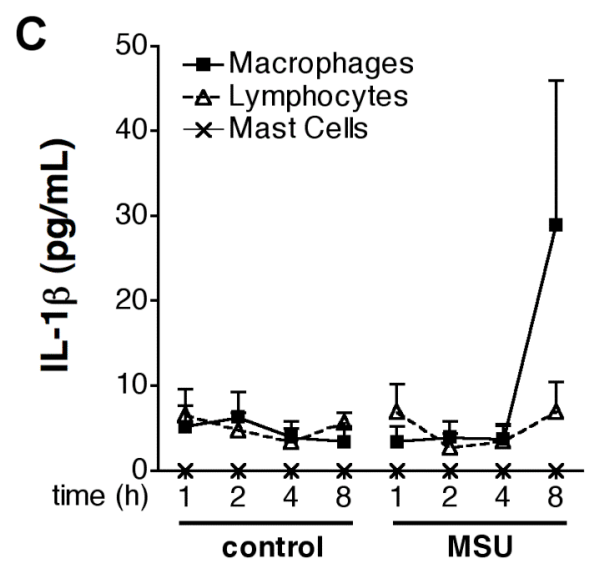

Figure 5.9 Macrophages are the major producers of MSU-induced proinflammatory cytokines in lavaged cells of naïve mice. Peritoneal leukocytes were harvested from naïve mice by peritoneal lavage $(3 \mathrm{~mL})$ and separated into subtypes by density gradient. Macrophages $\left(2 \times 10^{5}\right.$ cells, $\left.200 \mu \mathrm{L}\right)$, lymphocytes $(2 \mathrm{x}$ $10^{5}$ cells, $\left.200 \mu \mathrm{L}\right)$ and mast cells $\left(2 \times 10^{4}\right.$ cells, $\left.200 \mu \mathrm{L}\right)$ were exposed to MSU $(200 \mu \mathrm{g} / \mathrm{mL})$ and the cytokines measured by ELISA and BioPlex Array. Measurements performed in triplicate. Values represent mean + S.E.M. Results are representative of three separate experiments. Experiments were performed by E. Chia. 


\subsubsection{Investigating activation of macrophages following exposure to MSU.}

Although the results described above indicated that resident macrophages were a potential source of IL-1 $\beta$, TNF $\alpha$, and IL-6 in vivo, these cells were notably absent from the peritoneal lavage after MSU administration. The absence of cytokineproducing macrophages from the peritoneal lavage of MSU-treated mice led me to look at changes in macrophage numbers during the course of the inflammatory response to MSU. Flow cytometric analysis of the $\mathrm{F} 4 / 80^{\mathrm{hi}}$, Gr-1 ${ }^{-}, 7 / 4^{-}$resident macrophage population showed complete disappearance of this population from the peritoneal lavage 2 hours after MSU exposure (Fig. 5.10).

One possible reason for the lack of macrophage retrieval is the adherence of macrophages directly to MSU crystals. And indeed, after MSU treatment in vivo MSU became associated in clumps that appeared as a mesh-like glob of cells and crystals (Fig. 5.11A). Crystals could be visualised within the clumps using polarised light microscopy (Fig. 5.11B). However the density of these clumps made it difficult to identify different cell types within the clump using immunofluorescent markers. To determine the likelihood of resident macrophages adhering to MSU crystals resident leukocytes from the peritoneum of naïve mice were exposed to MSU in vitro and then stained for macrophages using F4/80. Resident peritoneal cells bound to MSU crystals, as indicated by Hoechst staining, and formed clumps of crystals and cells in vitro (Fig. 5.12). Cells within the mesh of crystals stained positively for F4/80 showing that the majority of the cells within the mesh were macrophages (Fig. 5.12). The results clearly showed that macrophages adhere to MSU following exposure to crystals and form clumps with crystals mimicking the clumping observed in vivo (Fig. 5.12; enlarged inset).

In addition to adhesion to crystals, adhesion to surrounding tissues is a common feature of macrophage activation and the absence of macrophages shortly after administration of inflammatory stimuli has been reported as the 'macrophage disappearance reaction' in other acute inflammatory models (Nelson and Boyden, 1963, Haskill and Becker, 1985, Melnicoff et al., 1989). This phenomenon may also have contributed to the reduction of retrievable macrophages from the peritoneum following MSU administration. 


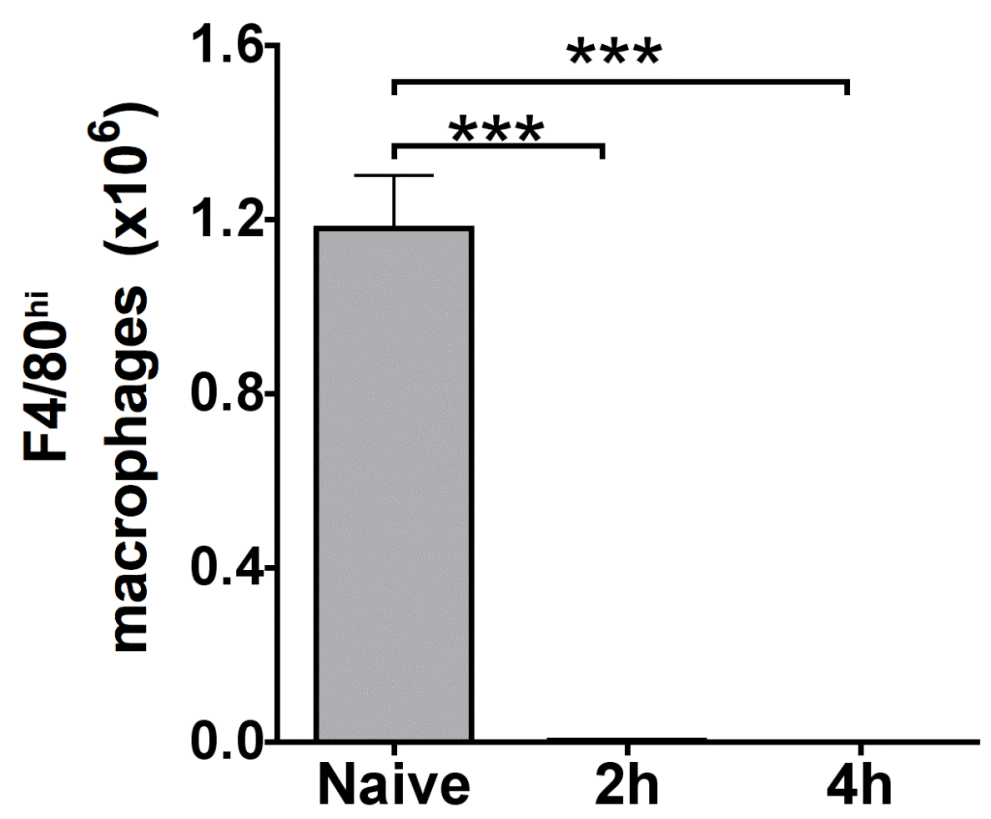

Figure 5.10 Resident macrophages disappear from the peritoneal lavage following MSU administration. Mice were treated with MSU i.p. (3mg; 0.5mL PBS) and at various timepoints mice were sacrificed. Cells in the peritoneal cavity were harvested by lavage (3mL PBS). Harvested peritoneal cells were analysed for the presence of $\mathrm{F} 4 / 80^{\mathrm{hi}}$ resident macrophages by FACS. Values represent mean + S.E.M. ( $\mathrm{n}=3$ mice per group). ${ }^{* * *}=P<0.001$ as determined by Student's $t$-test against the naïve time point. Results are representative of three separate experiments. 


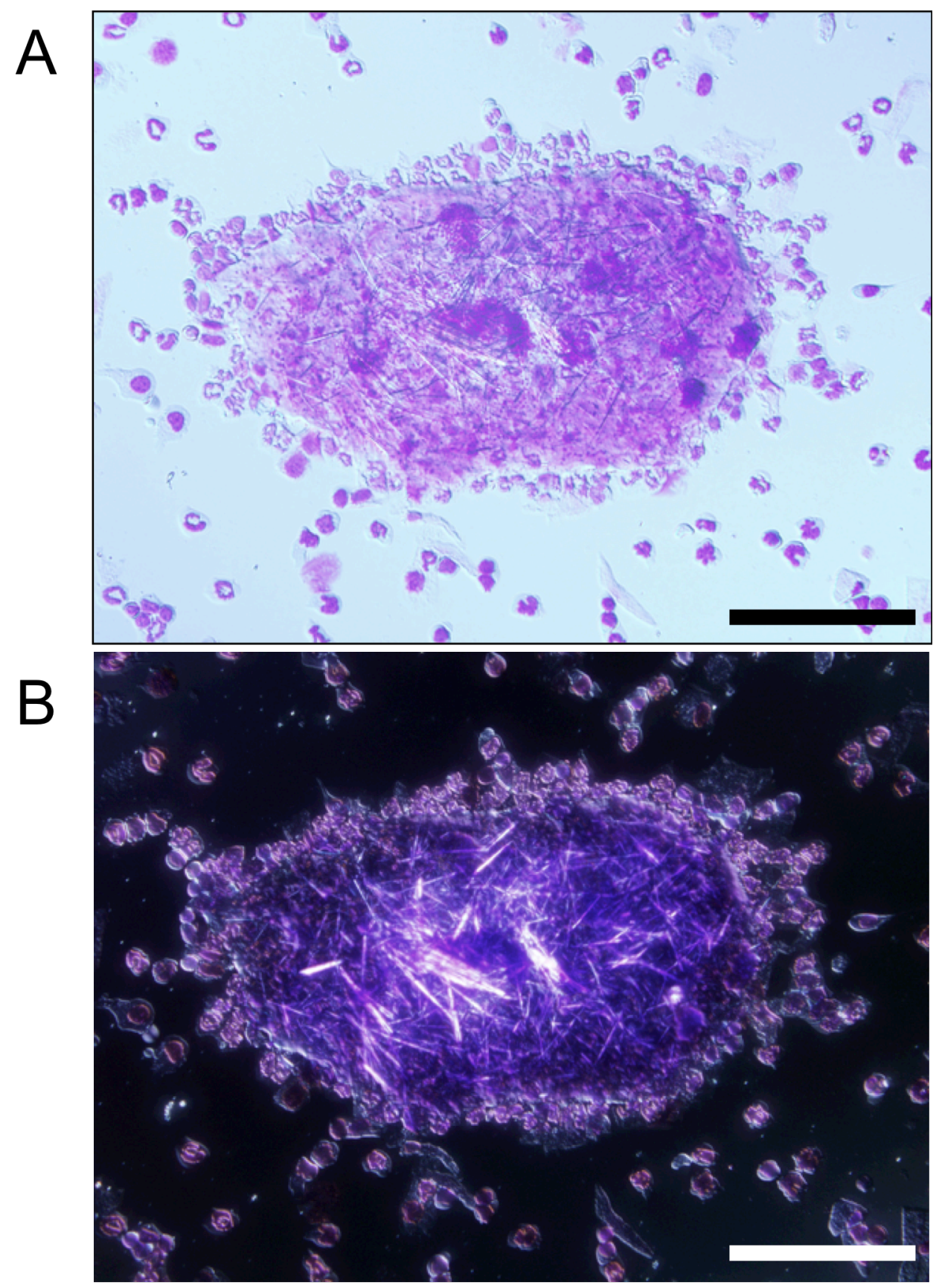

Figure 5.11 MSU crystals become associated with cells as clumps in vivo. Clumps found in the peritoneal wash of MSU-treated mice were sedimented by cytocentrifuge and stained with Diff-Quick. A, shows a clump of crystals under bright field. B, shows the same clump under polarized light. Original magnification 40x. Scale bar represents $100 \mu \mathrm{m}$. 


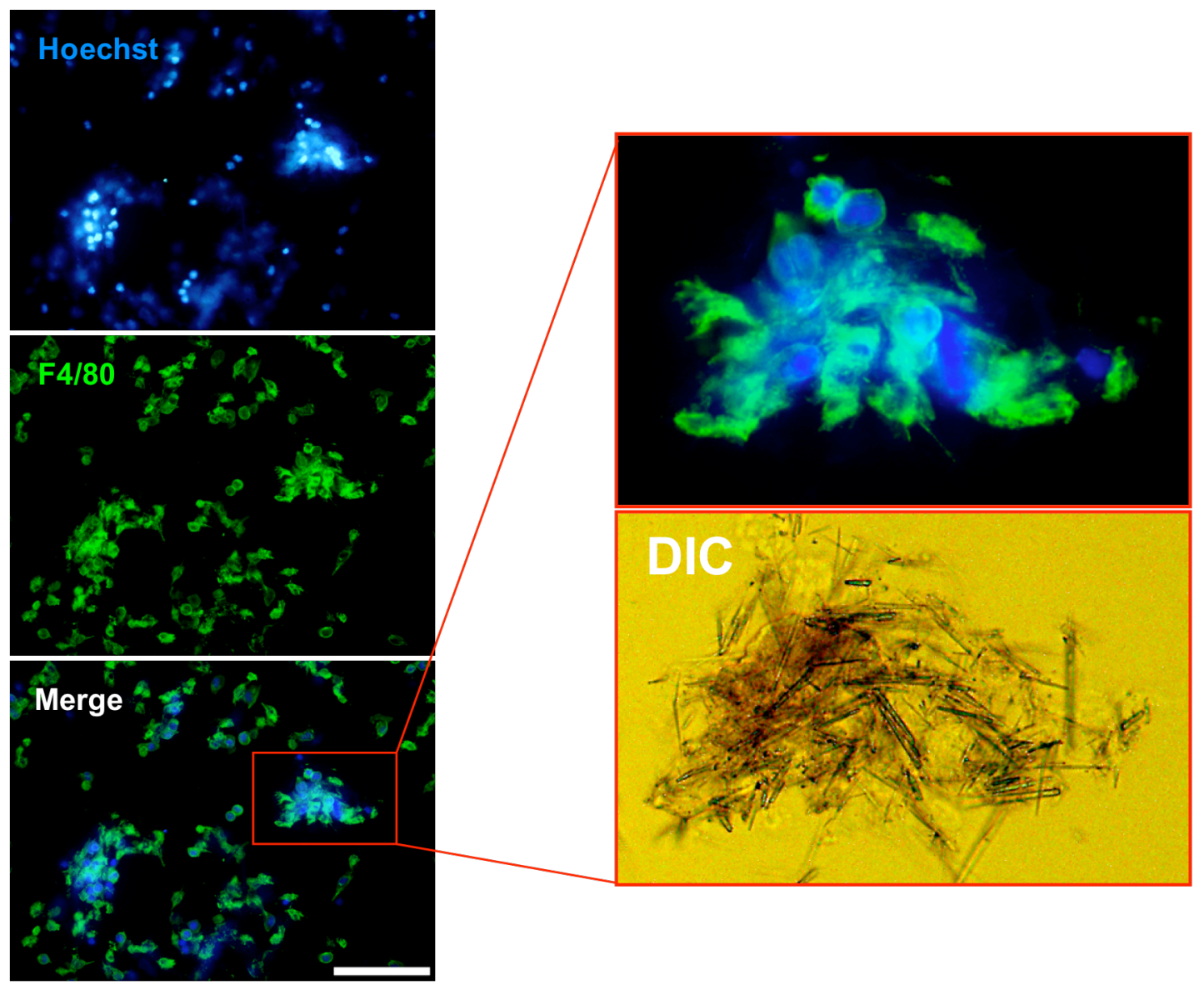

Figure 5.12 Resident macrophages associate with MSU crystals in vitro. Resident peritoneal leukocytes were harvested from naïve mice by lavage $(3 \mathrm{~mL}$ PBS) following which they were exposed to MSU in vitro. Cells were then stained for F4/80 (green) and the nuclear dye Hoechst 33342 (blue). The MSU/cell mesh within the red square is shown enlarged. MSU crystals within the MSU/cell mesh are also shown by differential interference contrast (DIC). Original magnification 40x. Scale bar represents $100 \mu \mathrm{m}$. Results are representative of three separate experiments. 
To confirm that macrophage adherence was occurring in vivo following MSU administration, visceral peritoneal membranes were isolated from mice four hours after MSU administration and stained for the presence of $\mathrm{F} 4 / 80^{\mathrm{hi}}, 7 / 4^{-}$resident macrophages using immunofluorescence. Following MSU treatment, adherent resident macrophages were observed on the peritoneal membrane (Fig. 5.13). No adherent macrophages were present on tissue from untreated mice, indicating that macrophage activation and adherence had occurred in response to treatment with MSU. As confirmation that adherent $\mathrm{F} 4 / 80^{\text {hi }}$ cells could produce cytokines to MSU, resident peritoneal macrophages were adhered to slides and then exposed to MSU. In vitro cultures of the naïve peritoneal cell population identified the $\mathrm{F} 4 / 80^{+}$resident macrophages as producing not only IL-6 but also TNF $\alpha$ and IL-1 $\beta$ in response to MSU exposure (Fig. 5.14) indicating that resident macrophages were a source of inflammatory cytokines following MSU treatment in vivo.

\subsubsection{Effect of macrophage depletion in vivo}

Next, I sought to confirm the importance of MSU-induced activation of resident macrophages to the inflammatory response in vivo by depleting the resident macrophage population in the peritoneum prior to MSU administration. Resident peritoneal macrophages were depleted using clodronate-loaded liposomes where liposome pre-treatment caused a complete reduction of $\mathrm{F} 4 / 80^{\mathrm{hi}}$ resident macrophages, with an overall 80 percent depletion of all F4/80 cells. The small proportion of remaining $\mathrm{F} 4 / 80^{\mathrm{lo}}$ cells observed represented a small number of infiltrating monocytes (Fig. 5.15A and 5.15C, 0h). Following MSU treatment, all $\mathrm{F} 4 / 80^{+}$cells were $\mathrm{F} 4 / 80^{\mathrm{lo}}$ monocytes, and the infiltration of these monocytes was unaffected in macrophage-depleted mice compared with non-depleted mice (Fig. 5.15C). As shown in Figures 5.15B and 5.15D, macrophage depletion was associated with a significant reduction in total cell numbers and neutrophil infiltration following MSU administration. Reductions in IL-6, IL-1 $\beta$ and MCP-1 production were also observed, while the level of TNF $\alpha$ was not affected (Fig. 5.16A). Cytokine levels also dropped in the serum, with a reduction in IL-6 and MCP-1 levels observed (Fig. 5.16B). These results confirmed that resident macrophages play an essential role in the inflammatory phase of gouty 

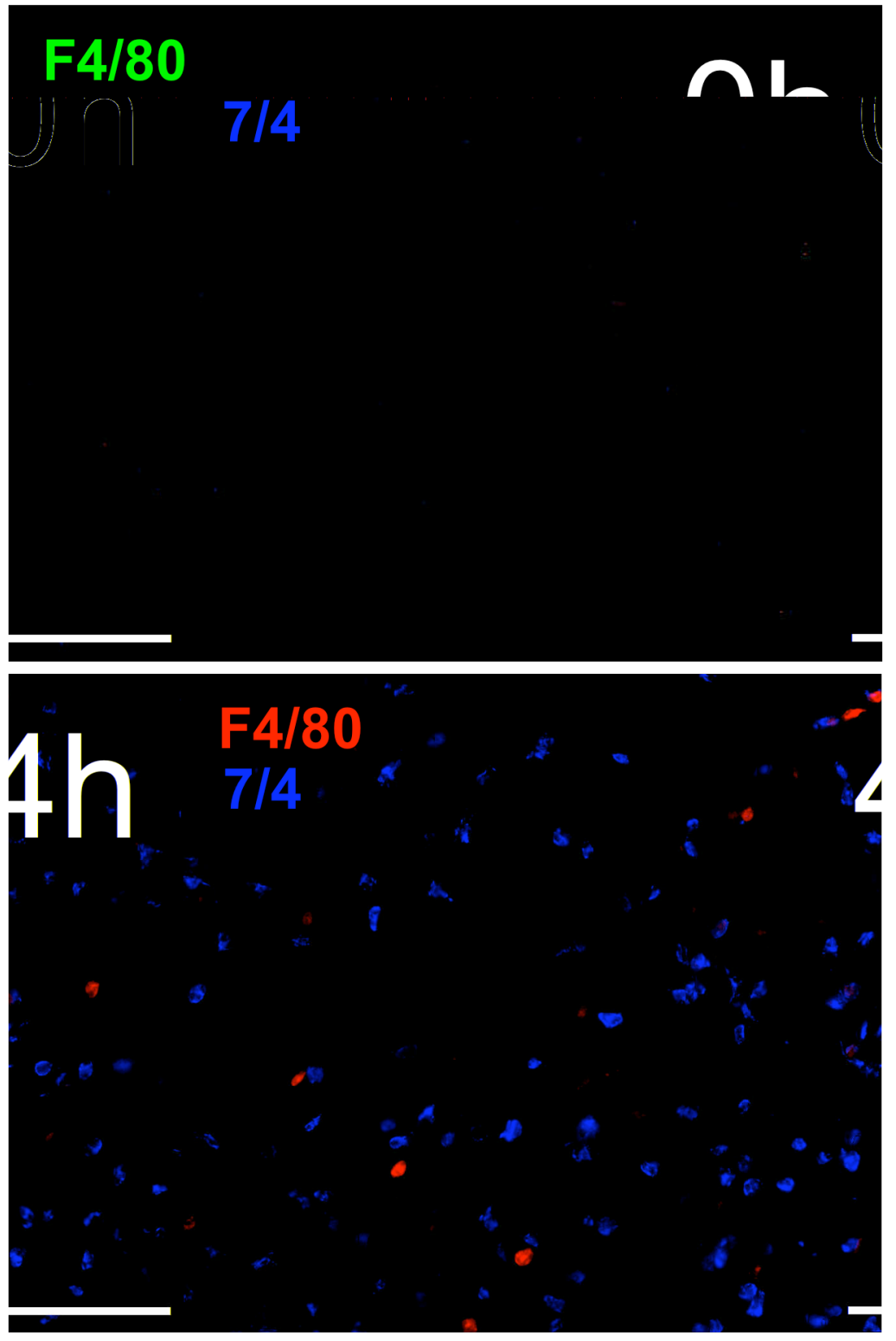

Figure 5.13 Resident macrophages adhere to the epithelial membrane in response to MSU. Mice received an i.p. injection of MSU (3mg; 0.5mL PBS) and after four hours the visceral epithelial lining of the peritoneum was harvested, fixed and then stained wrth anti-F4/80 and 7/4. Resident peritoneal cells appear as F4/80 $7 / 4^{-}$cells. Original magnification $40 x$. Scale bar represents $100 \mu \mathrm{m}$. Results are representative of three separate experiments. 

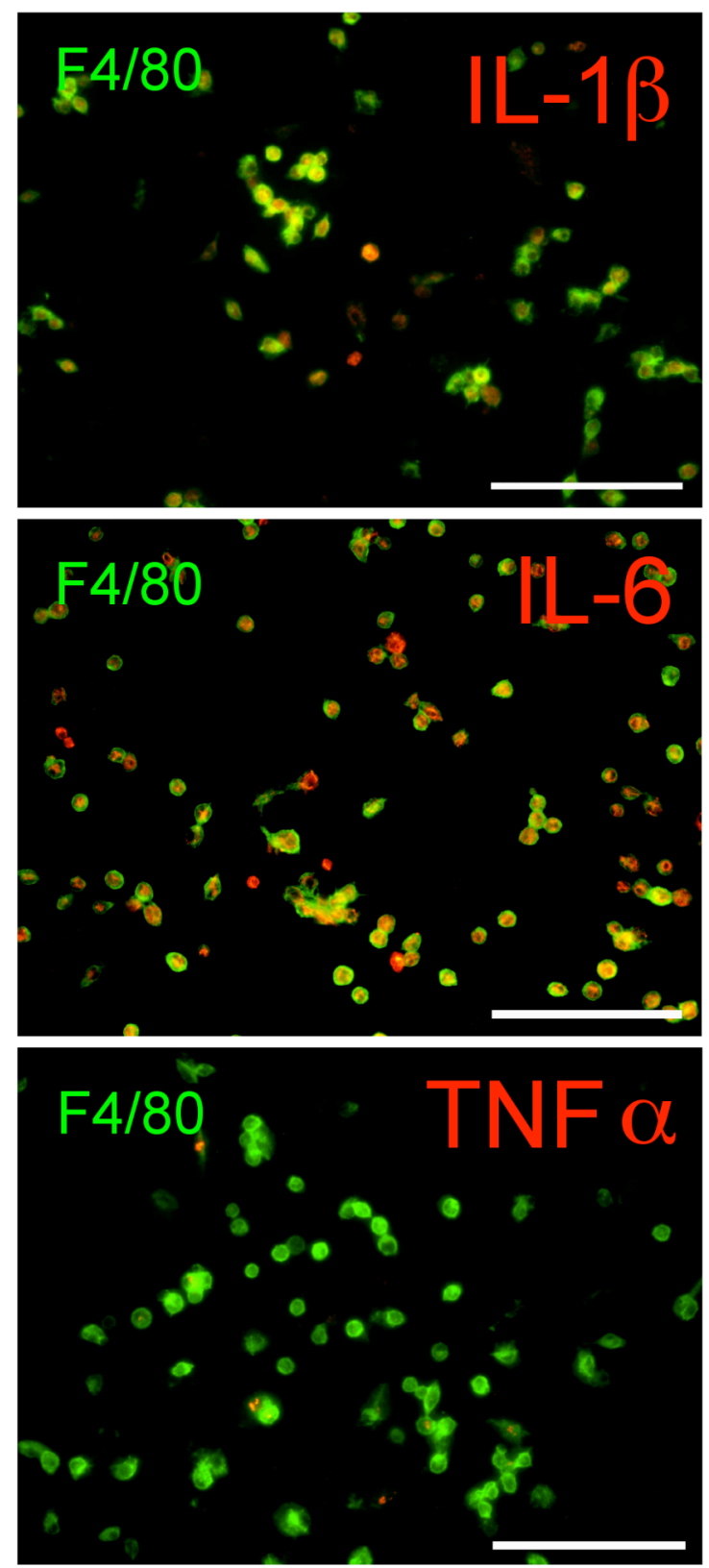

Figure 5.14 Resident macrophages are a source of pro-inflammatory cytokines in response to MSU. Resident peritoneal cells were harvested from naïve mice by lavage and exposed to $200 \mu \mathrm{g} / \mathrm{mL}$ MSU in the presence of GolgiStop for 4 hours. Production of the cytokines (red) IL-1 $\beta$, TNF $\alpha$ and IL- 6 by resident macrophages (anti F4/80; green) were then determined by fluorescent antibody staining. Original magnification 40x. Scale bar represents $100 \mu \mathrm{m}$. Results representative of three separate experiments. 
Figure 5.15 Depletion of resident macrophages inhibits MSU-induced neutrophil infiltration. Mice were pre-treated with clodronate liposomes three days before being challenged with an i.p. injection of MSU ( $3 \mathrm{mg}$; 0.5mL PBS). Cells in the peritoneal cavity were harvested by lavage (3mL PBS). A, The effect of the liposome depletion on resident macrophages was determined by FACS before an MSU challenge. $4 \mathrm{~h}$ and $8 \mathrm{~h}$ following MSU administration, the number of $\mathbf{B}$, total cells, $\mathbf{C}$, monocyte-macrophages and $\mathbf{D}$, neutrophils were determined in liposome treated and non-liposome treated mice. Light bars represent clodronate liposome-treated mice while dark bars represent undepleted control mice. Values represent mean \pm S.E.M. ( $\mathrm{n}=5$ mice per group). ${ }^{*}=P<0.05, * *=P<0.01$ and $* * *=P<0.001$ as determined by Student's $t$-test against the relevant indicated timepoint. Results are representative of three separate experiments. 
A

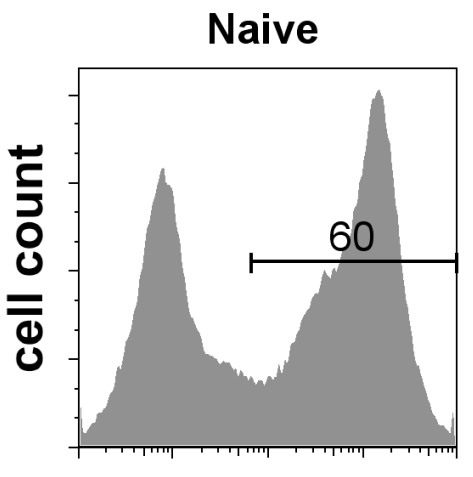

F4/80

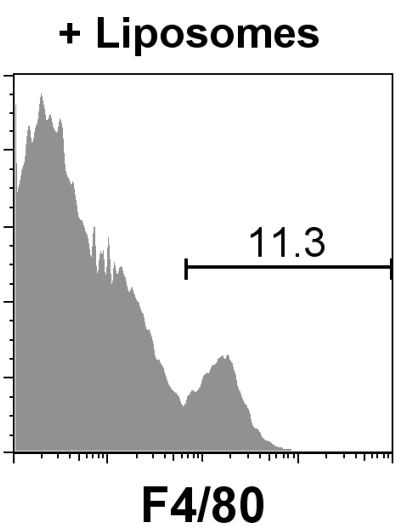

B

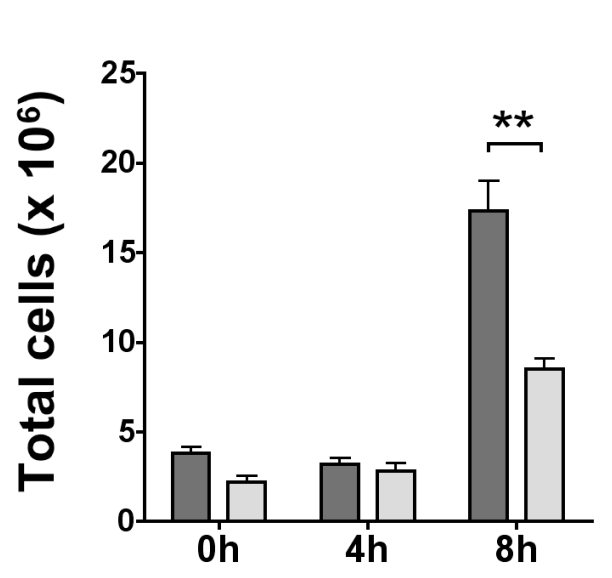

C

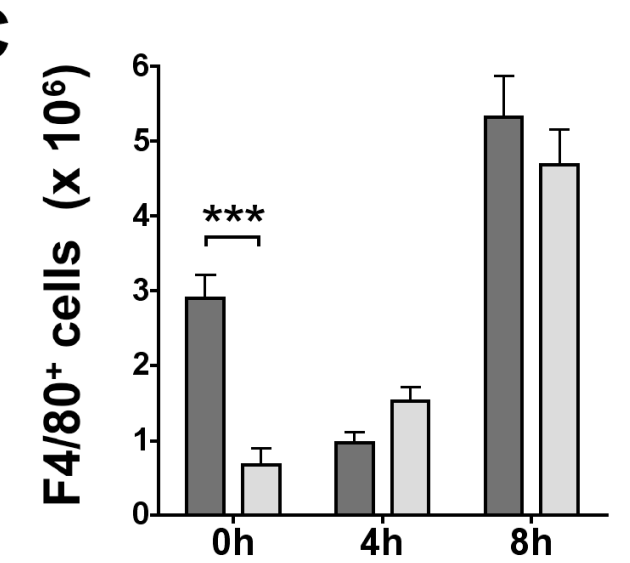

D

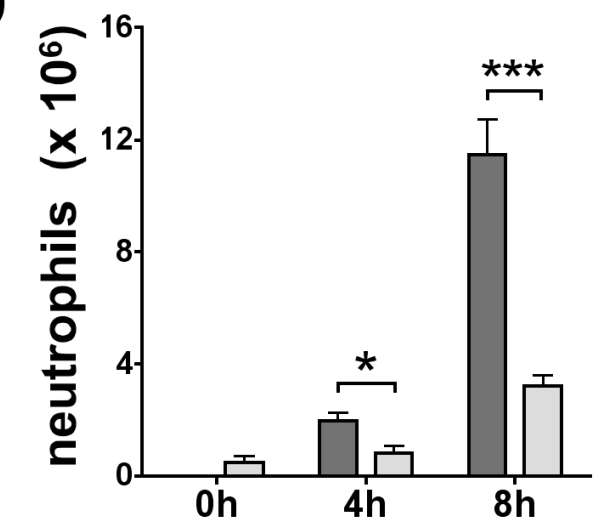



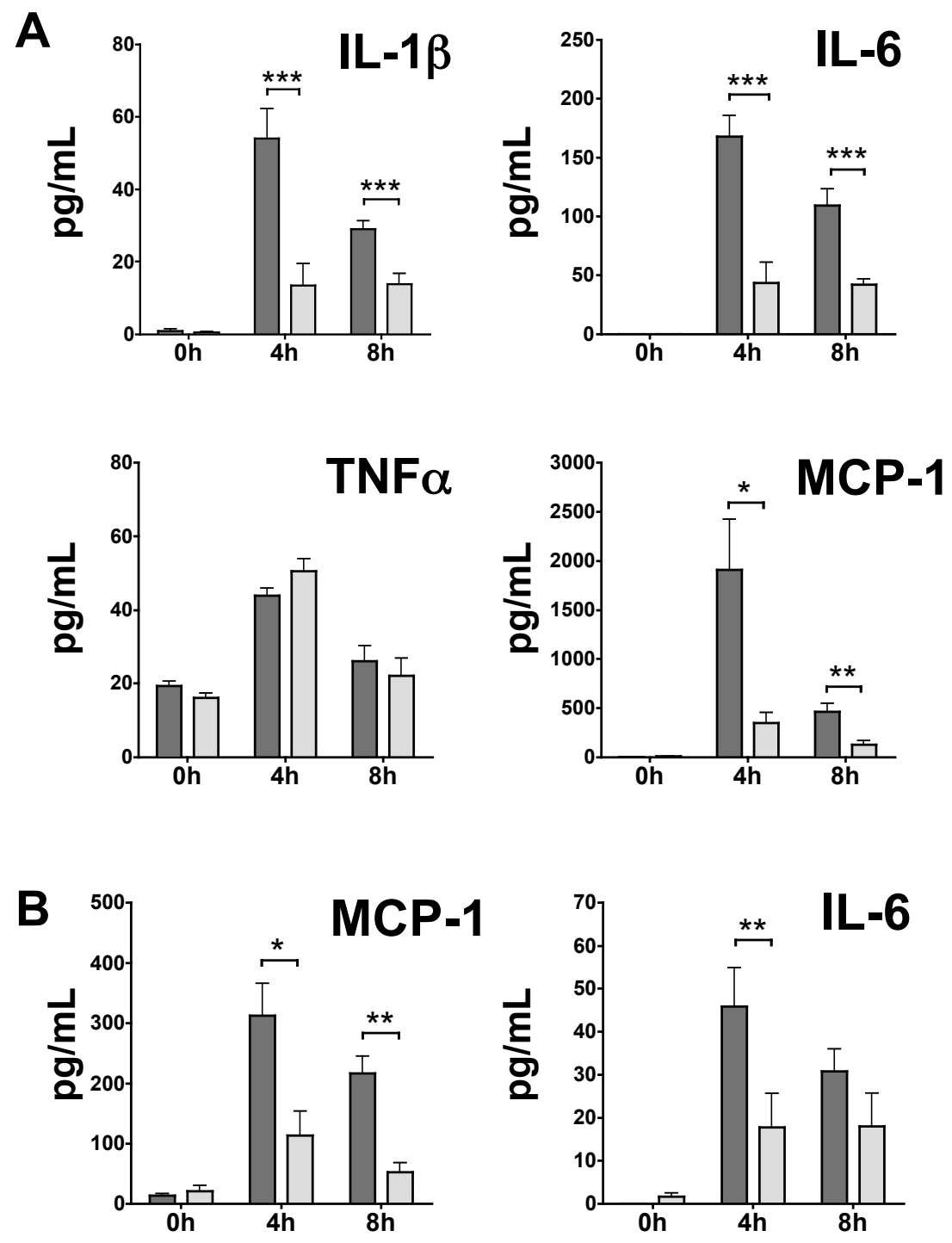

Figure 5.16 Depletion of resident macrophages inhibits MSU-induced cytokine production. Mice were pre-treated with clodronate liposomes three days before being challenged with an i.p. injection of MSU (3mg; $0.5 \mathrm{~mL}$ PBS). The peritoneal cavity was washed by lavage ( $3 \mathrm{~mL}$ PBS). A. Cytokine levels in the peritoneal lavage fluid. B. Cytokine levels in the circulating blood. Light bars represent clodronate liposome-treated mice while dark bars represent undepleted control mice. Values represent mean \pm S.E.M. $\left(\mathrm{n}=5\right.$ mice per group). ${ }^{*}=P<0.05, * *=P<0.01$ and $* * *=P<0.001$ as determined by Student's $t$-test against the relevant indicated control. Results are representative of three separate experiments. 
inflammation, producing key pro-inflammatory cytokines and recruiting of neutrophils.

\subsubsection{Differential cytokine production by peritoneal leukocytes and peritoneal epithelial cells}

The macrophage depletion studies above showed that although macrophages were responsible for recruiting neutrophils in MSU-induced inflammation they were not responsible for monocyte recruitment, which was unaffected by macrophage depletion (Fig. 5.15C and 5.15D). This indicated that monocyte recruitment in gout is controlled by a mechanism independent of macrophage activation.

Epithelial cells have been reported to respond to MSU crystals opening the possibility that non-hemopoietic cells may also be involved in the inflammatory response to MSU (Falasca et al., 1993). It is possible that epithelial cells may have contributed to the MSU-induced cytokines responsible for monocyte recruitment in vivo. However, the contribution of epithelial cells in the peritoneal gout model could not be determined in the previous experimental setup as epithelial cells are not retrievable by lavage. Therefore, to determine the contribution of both resident peritoneal epithelial cells and peritoneal leukocytes to MSU-induced cytokine production, whole peritoneal tissue and peritoneal leukocyte cells, harvested from naïve mice, were exposed to MSU both separately and together. The length of culture was limited to eight hours to coincide with the period at which maximum cytokine production was observed in vivo.

A differential expression of cytokine production was observed between peritoneal leukocytes and epithelial cells. Leukocytes produced TNF $\alpha$, IL-1 $\beta$ and IL-6 in response to MSU, but only showed a modest increase in MCP-1; while epithelial tissue exposed to MSU did not produce TNF $\alpha$, IL-1 $\beta$ and IL- 6 but produced MCP-1 (Fig. 5.17). Cytokine production was not increased when both cell types were cocultured and then exposed to MSU, which meant that there was no overlap or 
Figure 5.17 Differential production of pro-inflammatory cytokines by peritoneal leukocytes and membrane following MSU exposure. Peritoneal leukocytes were harvested from naïve mice by peritoneal lavage $(3 \mathrm{~mL}$ PBS) and the peritoneal membrane excised. Peritoneal leukocytes and peritoneal membrane were cultured together or separately in the presence $(+)$ or absence (-) of MSU $(200 \mu \mathrm{g} / \mathrm{mL})$ for $8 \mathrm{~h}$ following which the levels of A, IL-6 B, TNF $\alpha, \mathbf{C}, \mathrm{IL}-1 \beta$ and D, MCP-1 were determined by ELISA and BioPlex Array. Measurements performed in triplicate. Values represent mean \pm S.E.M. $*=P<0.05, * *=P<0.01$ and $* * *=P$ $<0.001$ as determined by Student's $t$-test against the relevant indicated control. Results are representative of three separate experiments. 


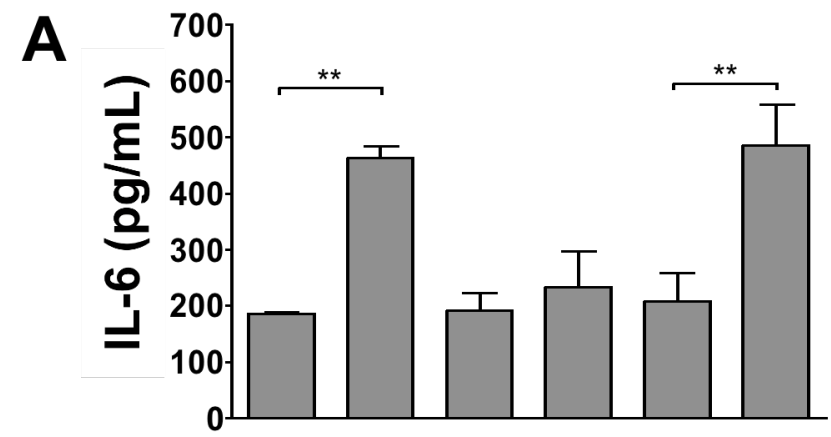

B

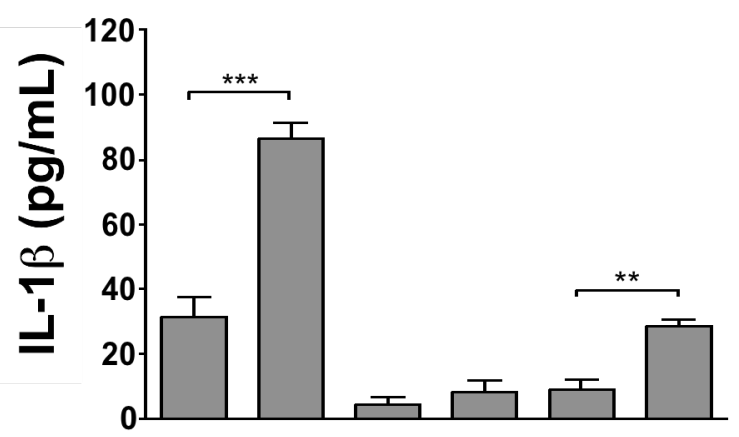

C

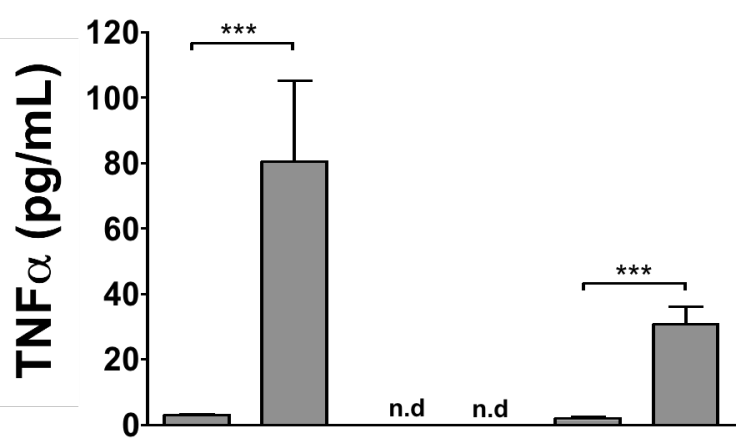

D

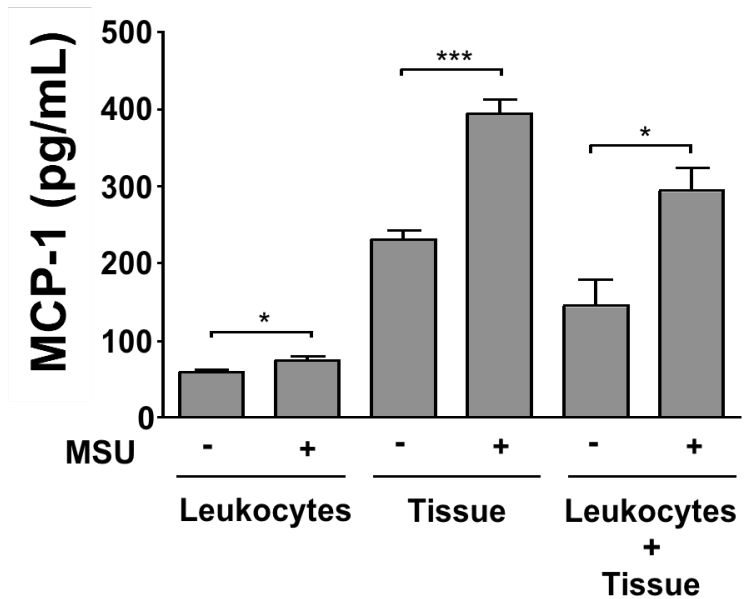


synergy in cytokine production by leukocytes versus epithelial cells. In some instances the cytokine response in combined leukocytes and epithelial cell cultures was reduced. However, examination of plates that had been inverted to allow the contact of leukocytes with tissue during culture revealed that a number of leukocytes had adhered to the bottom of the wells before plate inversion. Hence, when the plates were inverted these cells were excluded from the culture, resulting in diminished cytokine levels.

These results indicate that both leukocytes and epithelial cells in the peritoneum are involved in the production of pro-inflammatory cytokines in the initial phase of the inflammatory response, but produce different sets of cytokines. Resident peritoneal leukocytes produce IL-6, IL-1 $\beta$ and TNF $\alpha$ in response to MSU, while epithelial cells produce MCP-1.

The epithelial cell type present on peritoneal tissue is the mesothelial cell. To confirm that mesothelial cells were a source of MCP-1 following MSU stimulation, mesothelial cells were harvested from the peritoneum and cultured in vitro. Mesothelial cells were large and had a cobbled appearance consistent with previously reported literature (Herrick and Mutsaers, 2004)(Fig. 5.18A). As has been reported previously with cultured peritoneal tissue (Riese et al., 1999), culturing of mesothelial cells produced a background level of MCP-1 (Fig 5.18B). Following exposure to MSU crystals, mesothelial cells increased MCP-1 production, further implicating these cells as a source of MCP-1 in the MSU-stimulated peritoneum (Fig. 5.18B).

\subsection{Discussion}

The results presented in this chapter show that resident tissue macrophages play a significant role in the onset of acute gout. Resident macrophages are a key source of pro-inflammatory cytokines, including IL-1 $\beta$, in acute gout attacks. The key indication of the importance of resident macrophages was demonstrated when macrophages were selectively depleted. Not only did this lead to a reduction in 
A

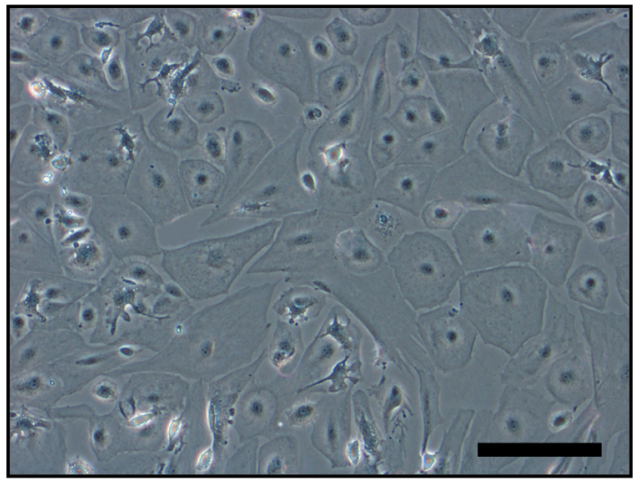

B

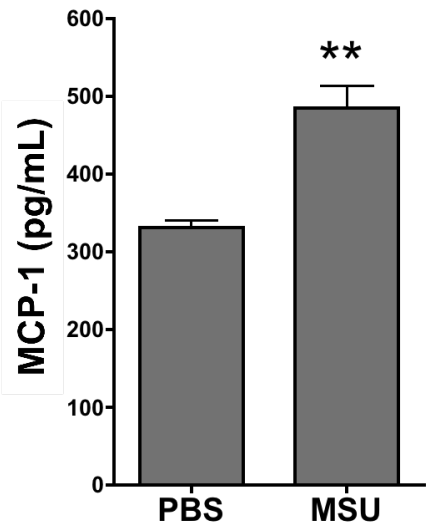

Figure 5.18 Mesothelial cells produce MCP-1 in response to MSU. Peritoneal mesothelial cells were harvested from naïve mice by in vivo trypsinization and cultured in vitro. A. Phase contrast picture of peritoneal monolayers in culture. Original magnification 40x. Scale bar represents $100 \mu \mathrm{m}$. B. MCP-1 production of mesothelial cells following exposure to $500 \mu \mathrm{g} / \mathrm{mL}$ MSU in vitro. Measurements performed in triplicate. Values represent mean \pm S.E.M. Results are representative of three separate experiments. Figure 5.18A was prepared by W. Martin while the experiments for $5.18 \mathrm{~B}$ were performed by $\mathrm{T}$. Liu. 
IL-1 $\beta$ and IL-6 but also to a significant reduction in neutrophil infiltration, demonstrating that macrophages do not only mediate cytokine responses in gout, they play a major role in orchestrating the neutrophil response.

Previous studies investigating the activation of monocytes in vitro have indicated that monocytes induce a pro-inflammatory response to MSU. It has therefore been hypothesised that monocytes aid in driving the inflammatory phase of gout. Contrary to expectations, the monocyte population recruited by MSU did not contribute to the early pro-inflammatory response in vivo nor were these monocytes responsive to MSU restimulation ex vivo. Since MSU crystals in the peritoneum form a mesh of cells and crystals, it could be argued that infiltrating monocytes may not make sufficient contact with crystals in vivo and, as a result, remain unactivated. However, this does not explain why the monocytes from MSU-treated peritoneal cavity were unresponsive to MSU ex vivo, despite being responsive to other inflammatory stimuli such as LPS. In fact, the lack of monocyte responsiveness to MSU may be a general feature of recruited monocytes, as monocytes recruited by thioglycollate stimulation showed a similar lack of responsiveness. The general absence of a pro-inflammatory response by infiltrating monocytes to MSU provides strong evidence that the infiltrating monocytes are not driving the early inflammatory phase in MSU-induced inflammation during the period of high proinflammatory cytokine production and neutrophil infiltration.

These findings were surprising in light of data showing that early leukocyte infiltrates from cantharidin-induced blisters produced pro-inflammatory cytokines following MSU-restimulation ex vivo (Yagnik et al., 2004). However, in addition to recruiting cells with an alternative inflammatory stimulus, the cantharidin study looked at later stage cellular infiltrates (16h compared to $4-8 \mathrm{~h}$ ) that likely differ in cellular composition and phenotype compared with the initiation phase of MSUinduced inflammation. Since the phenotype of the cells was not determined, it is not possible to determine whether these cells were representative of an early recruited phenotype. The use of the peritoneal model allowed extensive profiling of the rising and then resolving response, enabling cells from the appropriate stage of the inflammatory response to be studied. In addition, unlike the cantharidin study, cells 
recruited in the in vivo gout model were clearly identified by the phenotypic markers Gr-1, 7/4 and F4/80, allowing the contribution of discrete cell populations to be determined. Therefore, phenotypic differences between in vivo MSU-recruited monocytes and the monocytes derived by various other means in previous studies likely explain why the results presented in this thesis differ from earlier in vitro data.

Like monocytes, neutrophils did not produce IL-1 $\beta$, TNF $\alpha$, IL-6 or MCP-1 in response to MSU crystals. This does not exclude an inflammatory role for neutrophils in gout, as neutrophils produce superoxide in vivo when recruited in response to MSU (Chapter 3). The lack of classical pro-inflammatory cytokine production indicates that their primary function may not be the secretion of these cytokines towards driving inflammation in gout. However, other inflammatory mediators not measured within this study may also be involved, such as S100 proteins. Studying the involvement of mediators other than cytokines is a subject for further investigation.

The difference in responses of infiltrating monocytes to MSU and LPS in vitro suggests that monocyte recognition of MSU, unlike LPS, does not occur via TLR4. This is consistent with earlier work showing that knocking out the gene encoding TLR-4 does not ablate the inflammatory response to MSU (Chen et al., 2006). Further investigation into the differences in receptor expression between monocytes and macrophages may reveal new candidates for MSU recognition by mononuclear phagocytes.

The results in this chapter pinpoint the resident macrophage population as a primary source of pro-inflammatory cytokines in the early response to MSU exposure. This finding is consistent with a key involvement of resident macrophages in the initiation of other forms of acute inflammation (Richards et al., 1999, Knudsen et al., 2002, Cailhier et al., 2005). Previous gout research has mostly ignored the potential of this cell type to induce inflammation in favour of a possible anti-inflammatory effector function. 
Earlier in vitro studies have indicated that differentiated macrophages produce antiinflammatory cytokines such as TGF $\beta$ following MSU exposure (Yagnik et al., 2004). More recent literature has shown that MSU can activate differentiated macrophages, leading to the activation of the NALP3 inflammasome, the production of IL-1 $\beta$, the upregulation of TREM-1 and the production of the neutrophil chemokine KC (CXCL1) (Jaramillo et al., 2004a, Jaramillo et al., 2004b, Chen et al., 2006, Martinon et al., 2006, Murakami et al., 2006). These data are consistent with the in vivo findings described in this chapter whereby the resident macrophage population initiates inflammation in response to MSU. In the peritoneal model it appears that MSU-activated resident macrophages adhere to MSU crystals and to the surrounding tissues, produce pro-inflammatory cytokines and facilitate recruitment of neutrophils from the blood during the early inflammatory response. Because adherent resident macrophages are not readily recovered from lavage fluids, any contribution to disease activation and progression may well have gone unnoticed in favour of the contribution of more readily recoverable monocytes.

Depletion of macrophages had no effect on the recruitment of monocytes. This indicates that neither macrophages nor neutrophils are required for monocyte recruitment and that monocytes and neutrophils can be recruited via independent pathways, as has been observed in other inflammatory systems (Henderson et al., 2003). Differential cytokine production by peritoneal epithelium and leukocytes was characterised by epithelial cells producing MCP-1, and resident leukocytes producing IL-1 $\beta$, TNF $\alpha$ and IL-6. Given that MCP-1 is an important molecule in the recruitment of monocytes ( $\mathrm{Lu}$ et al., 1998) and IL-1 $\beta$ is key in neutrophil recruitment in gout, the prospect that epithelial cells and resident leukocytes may respectively control monocyte and neutrophil recruitment is a plausible interpretation of these data and warrants further investigation. There are clearly other pathways involved, as the macrophage depletion also led to a reduction in serum levels of both MCP-1 and IL-6 following MSU administration that cannot yet be explained. Nevertheless, these observations make important headway into dissecting the various pathways of activation involved in gout. 
Although resident leukocyte cells stimulated with MSU-crystals were also able to produce MCP-1 when cultured for $16 \mathrm{~h}$, this increase was not as significant when incubation time was limited to eight hours, a time period that reflected acute activation in vivo. In contrast, epithelial tissues produced large amounts of MCP-1 over the same length of time. This observation serves as a more technical caveat, emphasizing the importance of selecting appropriate culture times in vitro that reflect what is occurring in vivo in order to draw the most physiologically relevant conclusions.

A characteristic of acute gout attacks is the spontaneous resolution of inflammation even in the absence of clinical intervention. It is important to note that the proinflammatory response of the resident macrophage population to MSU observed in this study does not exclude differentiated macrophages from playing a role in the resolution of inflammation at a later point in time. The plasticity of the macrophage phenotype is well known and macrophage switching from a 'pro' to an 'anti' inflammatory phenotype over time remains a viable mechanism of action for resolution of acute inflammation. Although previous in vitro data implies that this may occur, functional switching in macrophages derived from resident macrophages or recruited monocytes has not been demonstrated in gout in vivo.

Mononuclear phagocytes are highly diverse in phenotype and function and these aspects often make it difficult to model their activities faithfully in vitro. The results presented here highlight the advantages of in vivo modelling in understanding the role of monocytes and macrophages in inducing gout. The use of in vivo derived phenotypes has clear advantages over using in vitro derived cell types as the true effector phenotype can be described in the direct context of the disease, and this approach has shown that infiltrating monocytes elicited by MSU in vivo behave contrary to monocytes derived by other methods. The observation of contrasting monocyte/macrophage responses also indicates that any clinical study investigating the function of monocytes or macrophages in gout will need to be select the correct cell type for study in order to yield any useful data. 
In summary, the results presented in this chapter identify resident macrophages - not infiltrating monocytes - as key cells initiating and driving inflammation in gout, suggesting the need to revisit the current model of acute gout induction.

Results of this chapter have been accepted for publication in Arthritis and Rheumatism. A draft of this paper has been inserted in the Appendix:

Resident macrophages: Initiating and driving early inflammation in an MSU-induced model of acute gout. W.Martin, M. Walton, J. Harper. Arthritis and Rheumatism. In press 2008 . 
Chapter 6

Profiling monocyte differentiation in acute MSU-induced inflammation 


\section{Chapter 6}

\section{Profiling monocyte differentiation in acute MSU-induced inflammation}

\subsection{Introduction}

The previous chapter revealed the unexpected phenomenon that monocytes do not produce pro-inflammatory cytokines (IL-1 $\beta, \mathrm{TNF} \alpha$, IL-6) in response to MSU during the early inflammatory phase of gout inflammation in vivo. The discovery of this lack of responsiveness of recruited monocytes is evidence that the use of blood monocytes to study gouty inflammation in vitro has led to a limited understanding of the role of monocytes in gout. Therefore, profiling of monocyte function and differentiation during an MSU response in vivo is required in order to understand the role of monocytes in gout more fully.

Monocytes in the blood consist of two principal subtypes that are broadly referred to as "inflammatory" monocytes and "resident" monocytes (Section 1.10.10). In humans, inflammatory monocytes are distinguished as $\mathrm{CD} 14^{\mathrm{hi}} \mathrm{CD} 16^{-}$while resident monocytes are $\mathrm{CD} 14^{\mathrm{lo}} \mathrm{CD} 16^{\text {hi }}$ (Passlick et al., 1989, Geissmann et al., 2003). There are a number of markers that identify the corresponding subtypes in mice, however in general they are distinguished by the expression of Gr-1 (Ly-6C), for which inflammatory monocytes are Gr- $1^{+}$and resident monocytes are Gr- $1^{-}$(Geissmann et al., 2003).

The importance of this distinction is that both monocyte groups have different functions. Inflammatory monocytes are so called due to their recruitment during inflammation (Geissmann et al., 2003). The high expression of chemokine receptors (e.g. CCR2 and 7 and CXCR1 and 2) provides further evidence that this subtype is poised to respond to inflammatory signals following an insult and that they are functionally distinct from resident monocytes (Lu et al., 1998, Weber et al., 2000, Grage-Griebenow et al., 2001). Infiltration of resident monocytes appears to occur independent of inflammatory stimuli and is dependent on high expression of the fractalkine receptor $\mathrm{CX}_{3} \mathrm{CR} 1$ (Auffray et al., 2007). In connection with this, a newly 
described "patrolling" function has been observed in resident monocytes in the vasculature that is not dependent on inflammation (Auffray et al., 2007). Although resident monocytes can also make a contribution to an inflammatory response, the ability of these cells to be recruited in the absence of inflammation has led to the idea that these cells may be responsible for the replenishment of monocyte-derived resident cells under steady state conditions (Geissmann et al., 2003).

It is as yet unclear whether resident and inflammatory monocytes are the source of separate cell populations following differentiation. Both subtypes are able to differentiate into DCs or macrophages in vitro depending on the growth factors and cytokines present (Sallusto and Lanzavecchia, 1994, Sanchez-Torres et al., 2001, Sunderkotter et al., 2004). However, in vitro systems are artificial by nature and it is likely that other complex factors come into play in vivo, such as the combined effect of local soluble factors, and the influence of local tissue antigens (Leenen and Campbell, 1993). As the study of monocyte subtypes is a relatively new area of research, there are few in vivo studies that have been undertaken to explore monocyte differentiation potential. Research papers using L. monocytogenes and UV irradiation as inflammatory stimuli in mice have shown that inflammatory monocytes have a tendency to engage a DC differentiation program while resident monocytes engage a macrophage differentiation program (Geissmann et al., 2003, Ginhoux et al., 2006, Auffray et al., 2007).

In Chapter 5, I have shown that monocytes recruited during MSU-induced inflammation are Gr- $1^{+}$(inflammatory) monocytes. In fact, a study following the differentiation fate of monocytes in gout in vivo has never been undertaken; rather it has been assumed that infiltrating monocytes become anti-inflammatory macrophages (Yagnik et al., 2004). Although research into the subtypes of monocytes is still an emerging field, there are ample tools now available to follow the changes in the phenotype of monocytes through a combinatory approach using surface marker expression, histology, and functional assays.

Since monocytes infiltrate following an MSU insult in vivo and do not appear to be involved in the onset of inflammation, I asked the question as to whether monocytes instead play a role during the resolution phase of the inflammatory response. 
Clearance of apoptotic neutrophils by monocyte/macrophages is a phenomenon associated with the resolution of inflammation in general. Phagocytosis of apoptotic cells during resolution does not lead to the production of pro-inflammatory cytokines, in fact, cells which have taken up apoptotic neutrophils in vitro have been shown to actively inhibit the production of pro-inflammatory cytokines via a process that has been shown to rely, in part, on the production of TGF $\beta$ (Fadok et al., 1998). TGF $\beta$ production has been widely reported in association with the resolution phase of MSU-induced inflammation (Liote et al., 1996, Yagnik et al., 2004). Although in vitro studies have implicated macrophages as the source of TGF $\beta$ leading to resolution in gout, this has not been confirmed in vivo.

Taking all of these factors into account, the purpose of this chapter was to profile the differentiation of monocytes recruited in response to MSU. This research was designed to provide a description of the changing marker expression, morphology and function of differentiating monocytes and confirm whether recruited monocytes differentiated into macrophages. Further this study sought to determine whether differentiating monocytes play a role in the resolution of inflammation in gout.

\subsection{Results}

\subsubsection{Monocytes differentiation in vivo}

In order to provide a clear profile of monocytes over the course of a response, monocytes were stained for the myeloid differentiation markers $7 / 4, \mathrm{Gr}-1$, the monocyte-macrophage lineage marker $\mathrm{F} 4 / 80$ and the costimulatory activation molecule CD80. As in Chapter 5, newly recruited monocytes were identified as $\mathrm{F} 4 / 80^{\mathrm{lo}}, 7 / 4^{\mathrm{hi}}$ and $\mathrm{Gr}-1^{\mathrm{int}}$.

Monocytes showed a progressive downregulation of both $7 / 4$ and Gr-1 that was completely lost by $48 \mathrm{~h}$ (Fig. 6.1A and B). During this period, monocytes maintained 
A

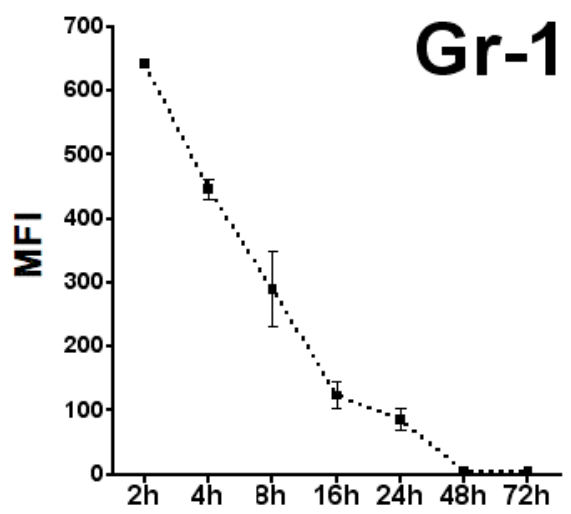

C

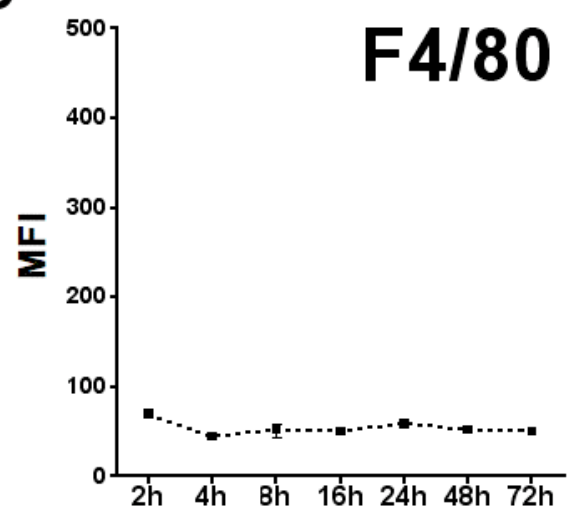

B

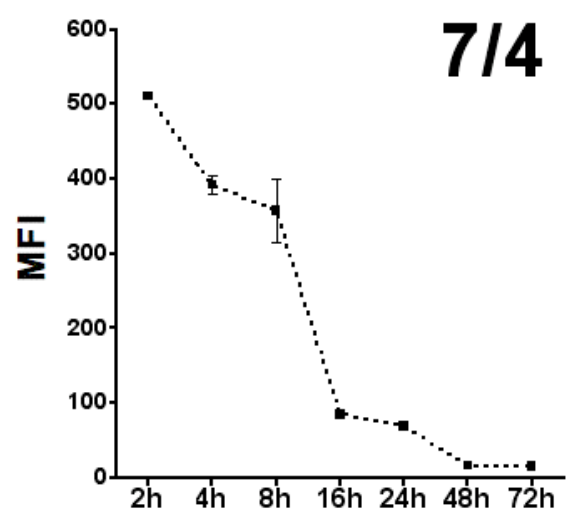

D

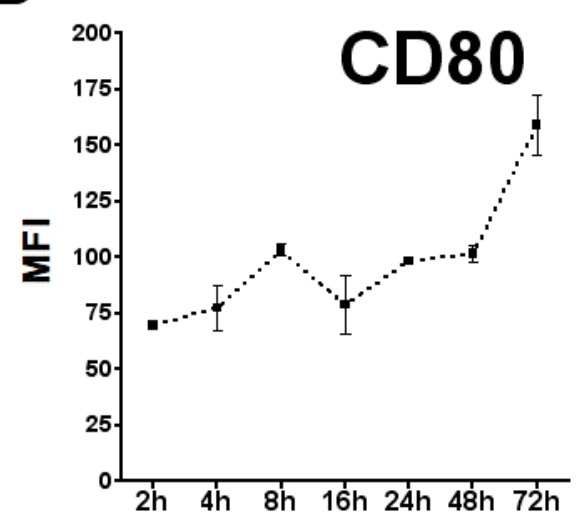

Figure 6.1 Marker expression on monocytes over the course of an MSU-induced acute inflammatory response.

Mice were treated with an i.p. injection of MSU (3mg MSU, 0.5mL PBS) and over the course of the response peritoneal exudate cells were harvested by lavage $(3 \mathrm{~mL}$ PBS). Harvested cells were prepared and stained for a number of monocytes markers. Monocytes were initially identified as $\mathrm{F} 4 / 80^{+}$cells, after which the expression levels of the myeloid differentiation antigens A. Gr-1 and B. 7/4 were determined over the course of the response. The expression level of C. F4/80 and the activation marker D. CD80 were also determined. Measurements were performed in triplicate. Values represent mean \pm S.E.M. Results are representative of three separate experiments. 
a constant low-level expression of F4/80 (Fig. 6.1C). After 72h, the expression of the activation marker $\mathrm{CD} 80$ on $\mathrm{F} 4 / 80^{\text {lo }}$ cells was significantly upregulated indicating that monocytes were differentiating towards a more pro-inflammatory phenotype.

\subsubsection{Mononuclear phagocyte morphology}

In order to view how infiltrating monocytes changed in morphology over time, 7/4, Gr-1 and F4/80 were used to identify and sort monocyte and macrophage populations over the course of the in vivo response. Resident peritoneal macrophages $\left(\mathrm{F} 4 / 80^{\mathrm{hi}}\right)$ were sorted from naïve mice; infiltrating monocytes (F4/80 ${ }^{\mathrm{lo}}$, Gr- $1^{\text {int }}, 7 / 4^{\text {hi }}$ ) were sorted from mice $8 \mathrm{~h}$ after an MSU injection; and immature macrophages $\left(\mathrm{F} 4 / 80^{\mathrm{lo}}, \mathrm{Gr}-1^{-}, 7 / 4^{-}\right)$were sorted from mice $48 \mathrm{~h}$ after MSU treatment.

Naïve resident macrophages were large cells, averaging $22 \mu \mathrm{m}$ in diameter on a cytospin slide, with rounded nuclei and a large cytoplasm (Fig. 6.2, left panel and Fig. 6.3). In contrast, infiltrating monocytes at $8 \mathrm{~h}$ were small in size averaging $13 \mu \mathrm{m}$ in diameter, contained only a small amount of cytoplasm and had more "bean" shaped nuclei (Fig. 6.2, center panel and Fig. 6.3). At 48h after MSU treatment, macrophages were at an intermediate size between monocytes and resident macrophages averaging $17 \mu \mathrm{m}$ in diameter (Fig. 6.2; right panel and Fig. 6.3). These cells exhibited the classical appearance of macrophages, including a rounded nucleus and a large cytoplasm. However, the size difference between resident and $48 \mathrm{~h}$ macrophages combined with their low F4/80 expression indicated that they were not identical resident macrophages and represented an intermediate phenotype between monocytes and resident macrophages.

Therefore it appeared that these three cell types represented three different stages of differentiation, which, for this study, I have termed as: mature macrophages, characterised by a large cell size and a high expression of F4/80; monocytes, characterised by the expression of the myeloid antigens Gr-1 and 7/4; and immature macrophages, characterised by a low expression of F4/80 without the expression of myeloid antigens, and with a cell size that is intermediate to monocytes and mature macrophages. 

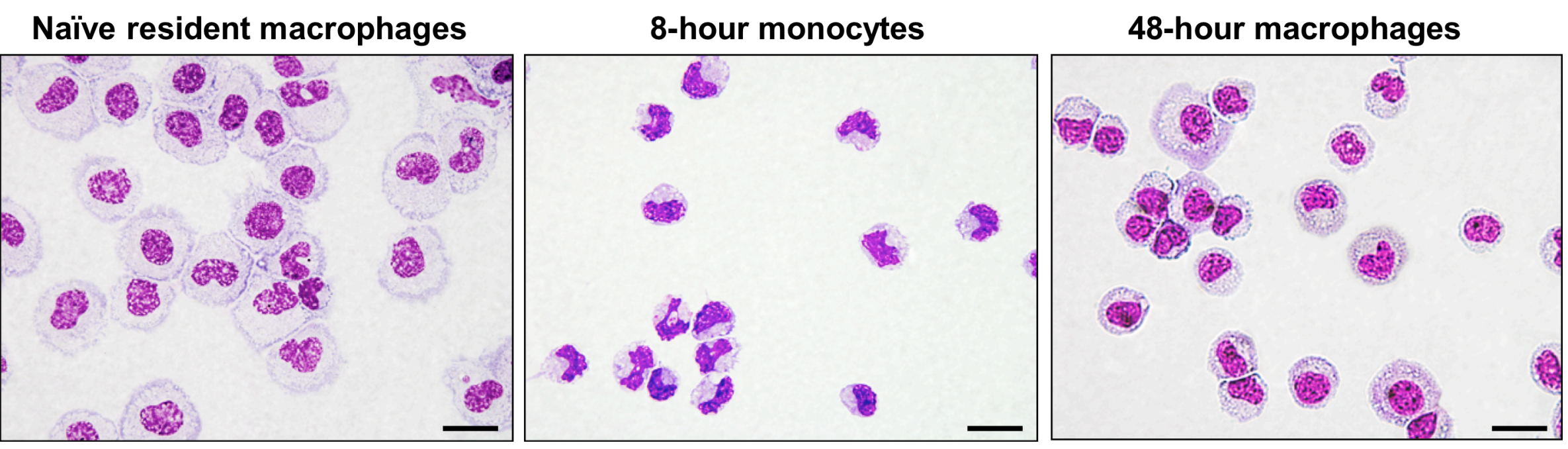

Figure 6.2 Morphology of mononuclear phagocytes over the course of the MSU-induced acute inflammatory response.

Mice were treated with an i.p. injection of MSU (3mg MSU, $0.5 \mathrm{~mL}$ PBS) and at various times cells were harvested from the peritoneum by lavage (3mL PBS). Monocyte-macrophages were then stained for the markers F4/80, Gr-1 and 7/4 and isolated by fluorescence activated cell sorting. Resident peritoneal macrophages $\left(\mathrm{F} 4 / 80^{\mathrm{hi}}, \mathrm{Gr}-1^{-}, 7 / 4^{-}\right.$cells) were isolated from naïve mice; monocytes $\left(\mathrm{F} 4 / 80^{\mathrm{lo}}, \mathrm{Gr}-1^{\mathrm{int}}, 7 / 4^{+}\right)$were isolated from mice $8 \mathrm{~h}$ after MSU administration; and immature macrophages (F4/80 $\left.{ }^{\mathrm{lo}}, \mathrm{Gr}-1^{-}, 7 / 4^{-}\right)$were isolated from mice 48h after MSU administration. Sorted cells were transferred onto slides by cytospin and stained with Diff-Quik for visualisation. Original magnification 100x. Scale bar represents $20 \mu \mathrm{m}$. 


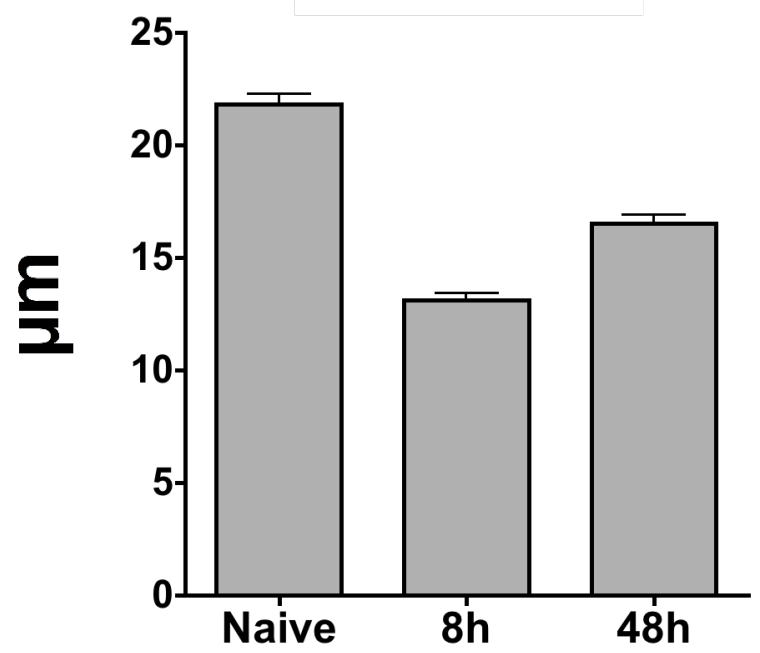

Figure 6.3 Cell width of mononuclear phagocytes over the course of the MSUinduced acute inflammatory response

The diameter of cells from Figure 6.2 were measured on cytospin slides using Olympus AnalySIS software. Results represent mean \pm S.E.M. 50 cells were measured for each variable. Results are representative of two separate experiments. 


\subsubsection{Expression of the dendritic cell marker CD11c}

The expression of F4/80 on immature macrophages indicated that these cells were most likely precursors for mature macrophages. However, it could not be ruled out that these monocytes may have been dendritic cell precursors that had not yet lost the expression of F4/80. To test this, I examined the expression of the common dendritic cell marker CD11c (Shortman and Liu, 2002) on cells over the course of the in vivo response. Very few cells in the peritoneum expressed CD11c $(<6 \%)$, with the vast majority of cells (78\%) expressing low F4/80 and no CD11c as far out as $48 \mathrm{~h}$ (Fig. 6.4). This observation provided evidence that these recruited monocytes were not becoming dendritic cells when recruited to gout inflammation.

\subsubsection{Tracking monocyte differentiation through cell labelling}

Next, I sought to confirm whether inflammatory monocytes were indeed the source of macrophage precursors by labelling the infiltrating inflammatory monocyte population and following their differentiation in vivo. To devise a way to label infiltrating monocytes, I took advantage of an observation previously noted in our lab. When the peritoneum was pre-treated with the dye PKH26 (which is selectively taken up by phagocytes) naïve resident macrophages were selectively stained with the dye (Fig. 6.5A). However, following MSU administration, these labelled resident macrophages were not retrievable and instead, a small proportion of infiltrating cells became labelled with dye as they entered the peritoneum in response to MSU (Fig. 6.5B). The level of dye uptake was much lower than in naïve resident macrophages (Fig. 6.5A and B) and these PKH26 $6^{\text {lo }}$ cells expressed Gr-1 and the 7/4 antigen indicating that inflammatory monocytes rather than neutrophils had taken up the dye (Fig. 6.5B). This staining approach allowed the $\mathrm{Gr}-1^{+}$monocytes to be followed over the course of their differentiation to confirm the cell type or types they became.

Using this labelling technique, PKH26-labelled cells were all $\mathrm{F} 4 / 80^{\mathrm{lo}}$ one day after MSU treatment, corresponding to monocytes (Fig. 6.6). These monocytes still expressed Gr-1, yet most of the monocytes were in the process of downregulating Gr-1 expression (Fig. 6.6; Day 1). There were no labelled cells that were F4/80 and 

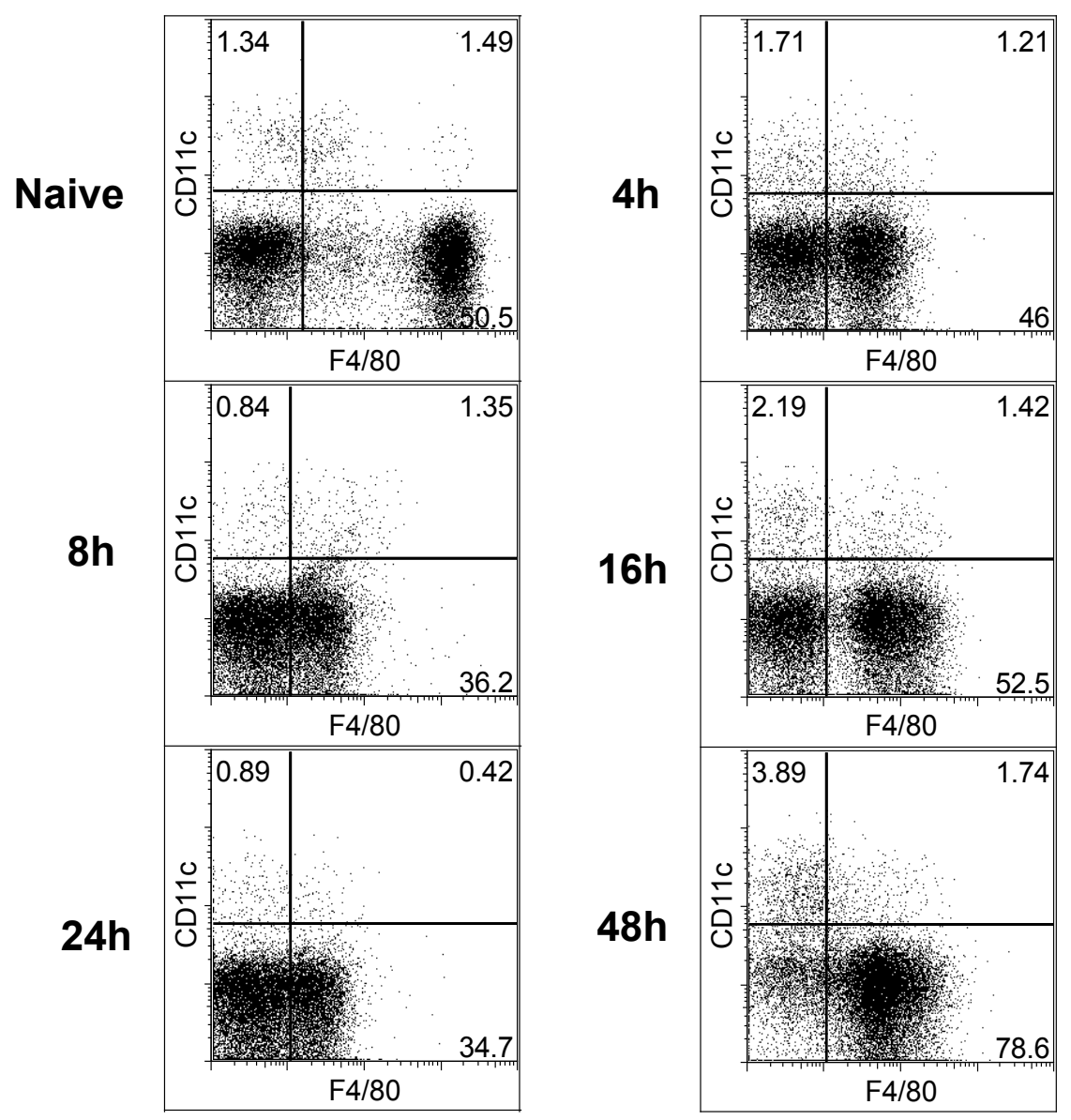

Figure 6.4 Expression of the dendritic cell marker CD11c over the course of an MSU-induced acute inflammatory response.

Mice were treated with an i.p. injection of MSU (3mg MSU, 0.5mL PBS) and over the course of the response peritoneal exudate cells were harvested by lavage $(3 \mathrm{~mL}$ PBS). Harvested cells were prepared and stained for CD11c and F4/80 and analysed by flow cytometry. Results are representative of three separate experiments. 
A

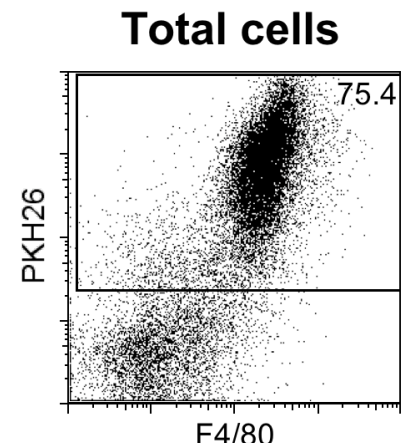

Naive
B

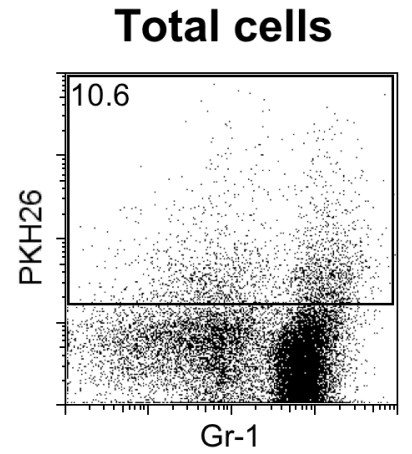

$\mathrm{PKH}^{+} 6^{+}$cells

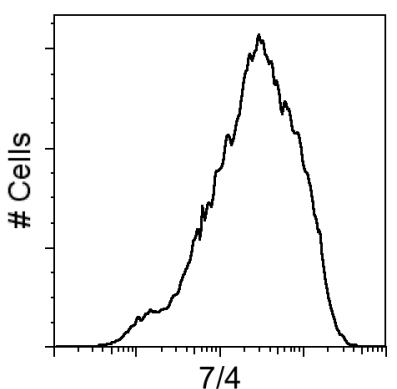

$8 \mathrm{~h}$

Figure 6.5 PKH26 labelling of mononuclear phagocytes.

Mice received an i.p. injection of the phagocyte dye PKH26 and one day later received an i.p. injection of MSU (3mg MSU, 0.5mL). Peritoneal exudate cells were harvested by peritoneal lavage $8 \mathrm{~h}$ after MSU treatment, stained for the surface markers and analysed by flow cytometry. A. PKH26 staining in cells of mice that had received the dye but were not injected with MSU.

B. Shows staining in peritoneal cells from mice $8 \mathrm{~h}$ after MSU treatment. Results are representative of three separate experiments. 

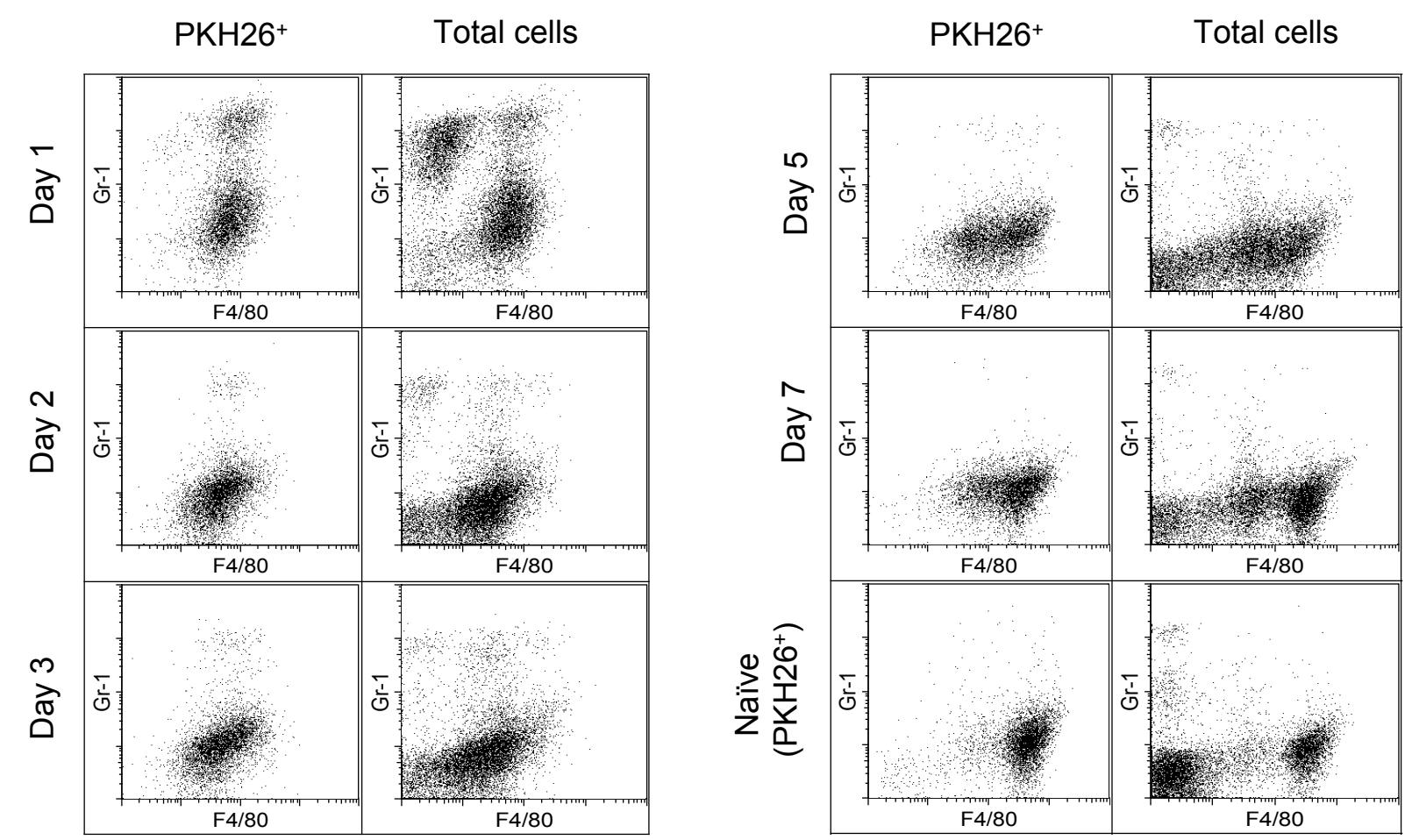

\section{Figure 6.6 Marker expression on $\mathrm{PKH}^{+}$monocytes over the course of an MSU-induced acute inflammatory response}

Mice received an i.p. injection of the phagocyte dye PKH26 and one day later received an i.p. injection of MSU (3mg MSU, 0.5mL). Peritoneal exudate cells were harvested by peritoneal lavage at various times after MSU treatment, stained for surface markers and analysed by flow cytometry. Results are representative of three separate experiments. 


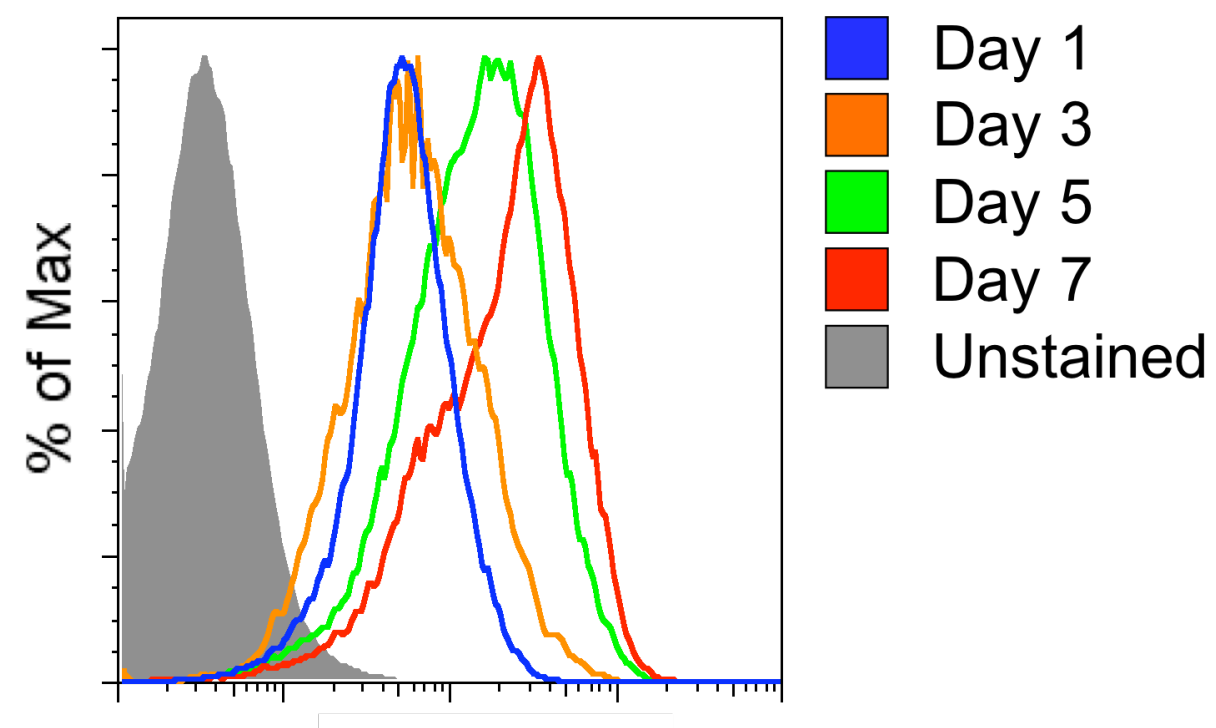

$\mathrm{F} 4 / 80[\mathrm{MFI}]$

Figure 6.7 F4/80 expression on differentiating monocytes over the course of an MSU-induced inflammatory response

Histogram of F/80 expression based on the monocyte-macrophages in Figure 6.6. Results representative of three separate experiments. 
Gr-1" showing that all monocytes were of the "inflammatory" rather than "resident" subtype. By day two, virtually all labelled cells were still F4/80 $0^{\text {lo }}$ but had lost the expression of Gr-1 (Fig. 6.6; Day 2). Interestingly, from day three after MSU treatment, labelled cells began to upregulate the expression of F4/80 (Figs. 6.6 and 6.7). After seven days there were two populations of PKH26 labelled cells: an $\mathrm{F} 4 / 80^{\text {lo }}$ population; and an $\mathrm{F} 4 / 80^{\mathrm{hi}}$ population comparable in expression level to resident macrophages (Fig. 6.6; Day 7 and Naive). These two populations indicated a differentiating, immature macrophage and a resident macrophage phenotype respectively.

These results demonstrated that in the gout model, inflammatory monocytes differentiated into macrophages, and were the primary source of macrophage precursors following a gout attack. The differentiation of macrophage precursors progressed through at least two detectable stages of differentiation. The first stage was from monocyte to immature macrophage, observed through the loss of the markers Gr-1 and 7/4. The second stage was from immature to mature macrophage observed through the change from $\mathrm{F} 4 / 80^{\mathrm{lo}}$ to $\mathrm{F} 4 / 80^{\mathrm{hi}}$ expression.

\subsubsection{Functional changes in differentiating monocytes}

Following evidence of monocyte differentiation to macrophages I next asked the question of whether there was a corresponding change in function in association with the observed changes in morphology and marker expression over the course of differentiation. Two classical functions of mononuclear phagocytes were chosen for investigation: the ability to produce chemical mediators in response to inflammatory stimuli and the capacity for phagocytosis.

\subsubsection{Production of pro-inflammatory cytokines in response to MSU}

In chapter 5 , I demonstrated that monocytes $\left(\mathrm{Gr}-1^{+}, 7 / 4^{+}, \mathrm{F} 4 / 80^{\mathrm{lo}}\right)$ isolated during the first 4-8h following MSU administration did not produce pro-inflammatory cytokines (IL-1 $\beta$, TNF $\alpha$, IL-6) following restimulation with MSU in vitro. Since these cells, with time, differentiated into macrophages, I wondered whether this corresponded to an altered response to MSU. 
To determine if this was indeed the case, total peritoneal cells were stimulated ex vivo from mice following in vivo MSU treatment. Although not all increases were statistically significant, total peritoneal cells from naïve mice showed a trend to increase pro-inflammatory cytokine production following stimulation with MSU (Fig. 6.8; 0h). Total peritoneal cells from mice treated with MSU for 4-16h did not exhibit an increase in cytokine production when re-stimulated with MSU ex vivo.

However, total peritoneal cells began to exhibit pro-inflammatory responses to MSU from mice treated with MSU for 24h. By $72 \mathrm{~h}$, the total peritoneal cells produced similar levels of pro-inflammatory cytokines to cells from naïve mice. This showed that the late stages of differentiation of recruited monocytes into macrophages were associated with increased responsiveness to MSU stimulation. Immature macrophages present in the peritoneum 24 to $72 \mathrm{~h}$ after MSU treatment exhibited pro-inflammatory responses to MSU that were similar to those of resident macrophages from naïve mice. To confirm that the newly differentiated macrophages were a source of the cytokine and chemokine production, F4/80expressing cells were purified from naïve mice and from mice $48 \mathrm{~h}$ after MSU treatment, and re-stimulated with MSU. The sorting procedure resulted in a high background cytokine production which may have prevented observing greater differences from MSU stimulation, nevertheless, both cell populations produced proinflammatory cytokines following restimulation with MSU ex vivo and exhibited a similar pattern of cytokine production to resident naïve macrophages, thus providing functional evidence that monocytes were differentiating into a pro-inflammatory macrophage phenotype (Fig. 6.9).

To confirm that these macrophages were pro-inflammatory in vivo, mice that had received an initial challenge with MSU were exposed to a second dose of MSU two days later. The inflammatory profile following in vivo restimulation was then compared to the profile of mice that had received a single dose of MSU. 


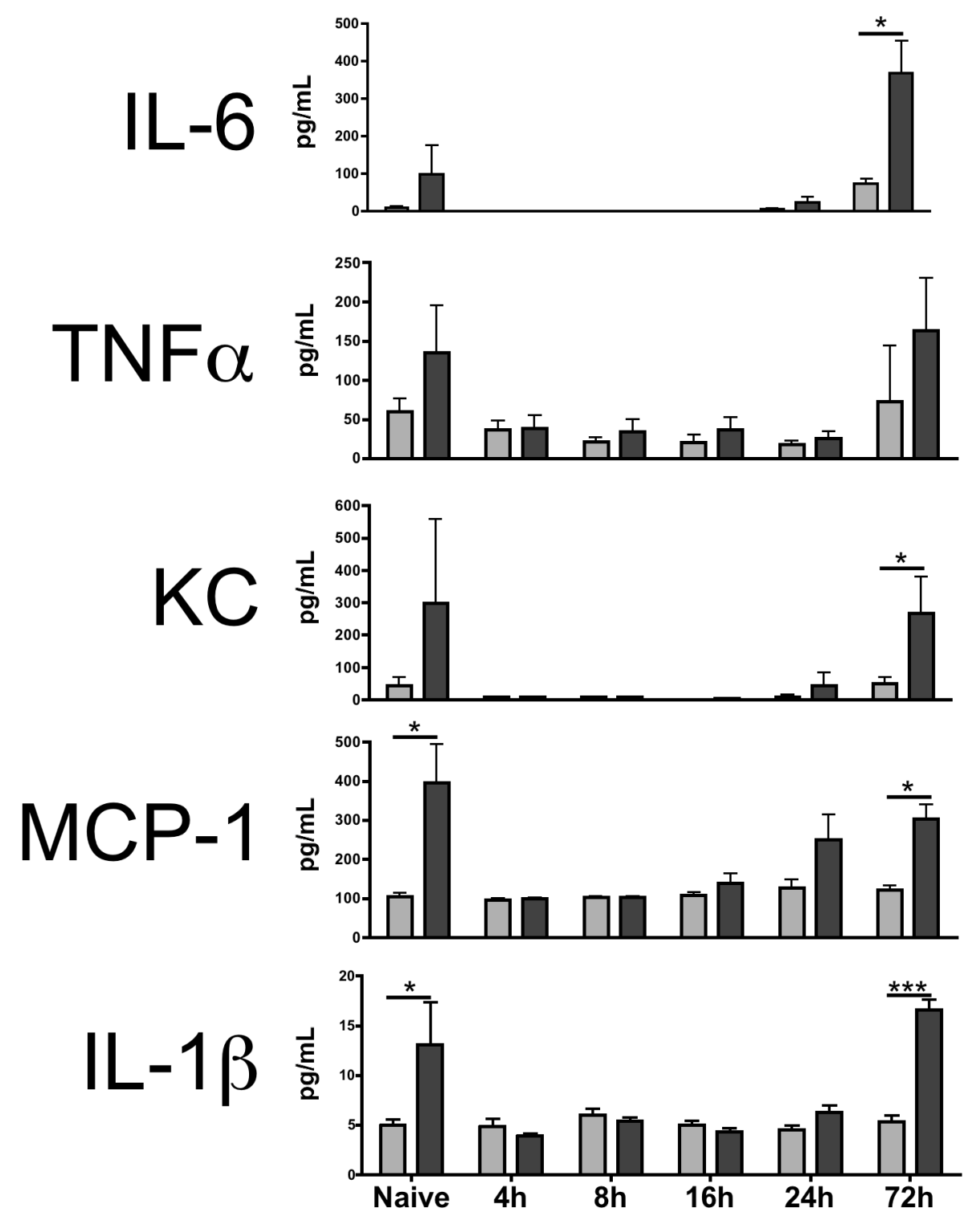

Figure 6.8 Responsiveness of peritoneal exudate cells to MSU restimulation

Mice were treated with an i.p. injection of MSU (3mg MSU, 0.5mL PBS) and over the course of the response peritoneal exudate cells were harvested by lavage $(3 \mathrm{~mL}$ PBS). Peritoneal exudate cells from each time point were suspended in culture medium $\left(1 \times 10^{6}\right.$ cells $\left./ \mathrm{mL}\right)$ and were treated with PBS (light bars) or with $200 \mu \mathrm{g} / \mathrm{mL}$ MSU (dark bars) to show the capacity of the cells present at each time point to produce pro-inflammatory cytokines in response to MSU. After 16h, supernatants were collected and cytokines analysed by ELISA or multiplex bead array. Measurements were performed in triplicate. Values represent mean \pm S.E.M. $*=P$ $<0.05$ and $* * *=P<0.001$ as determined by Student's $t$-test against the relevant indicated control. Results are representative of three separate experiments. 
Naive

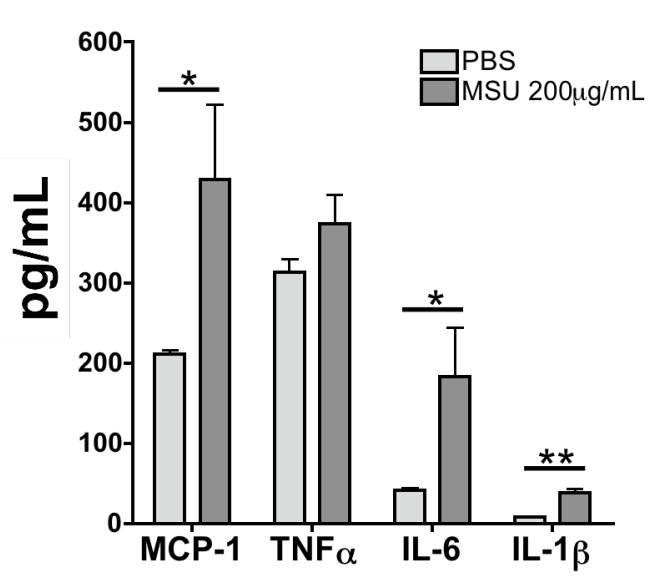

48-hour

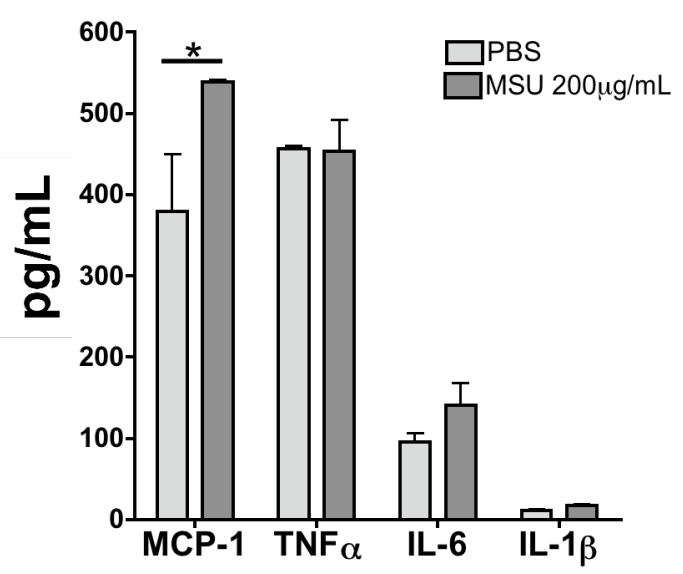

Figure 6.9 Stimulation of sorted macrophage populations ex vivo.

Macrophage populations were sorted by fluorescence activated cell sorting, and cultured for $16 \mathrm{~h}$ in the presence of PBS or MSU $(200 \mu \mathrm{g} / \mathrm{mL})$. Cytokine levels were then measured by ELISA and multiplex bead array. Left, Cytokine production from F4/80 naïve resident macrophages. Right, Cytokine production from F4/80 ${ }^{\text {lo }}$ immature macrophages harvested from mice $48 \mathrm{~h}$ after in vivo MSU treatment. Measurements were performed in duplicate and values represent mean \pm S.E.M. * ${ }^{*}$ $P<0.05$ and $P<0.01$ as determined by Student's $t$-test against the relevant indicated control. Results are representative of three separate experiments. 


\begin{tabular}{|c|c|c|}
\cline { 2 - 3 } \multicolumn{1}{c|}{} & Naive & 42h \\
\hline Macrophages & 84 & 92 \\
Lymphocytes & 14 & 4 \\
Neutrophils & 0 & 3 \\
Mast cells & $>1$ & $>1$ \\
Eosinophils & 0 & $>1$ \\
\hline
\end{tabular}

Table 6.1 Differential cell counts in peritoneal cells from naïve mice and from mice treated with MSU i.p. for $48 \mathrm{~h}$. 


\section{Figure 6.10 In vivo restimulation with a second injection of MSU}

Mice were treated with an i.p. injection of MSU (3mg MSU, 0.5mL PBS). Two days later, mice received a second injection of MSU i.p. and over the course of the response peritoneal exudate cells were harvested by lavage ( $3 \mathrm{~mL}$ PBS). (A) Total cells in mice receiving a single treatment (light grey bars) or two treatments of MSU (dark grey bars) were determined, as well as (B) monocyte-macrophages (C) and neutrophils. Lavage fluid was analysed for TNF $\alpha$ IL-6 and MCP-1 (D) by cytometric bead array. Measurements were performed in triplicate. Values represent mean \pm S.E.M. 
A

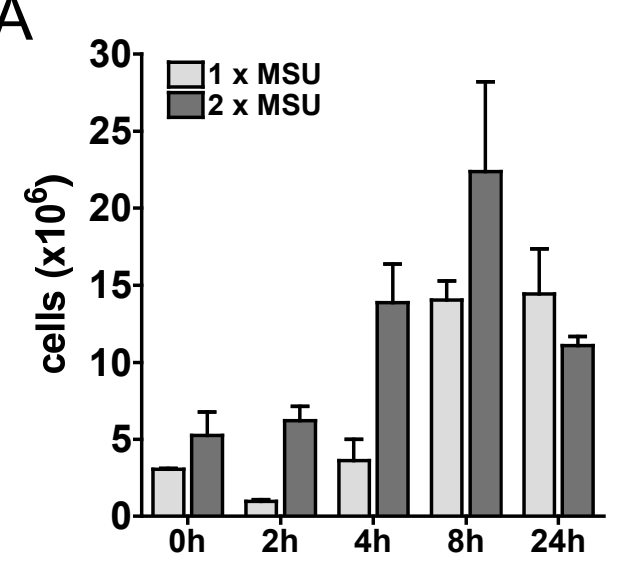

D

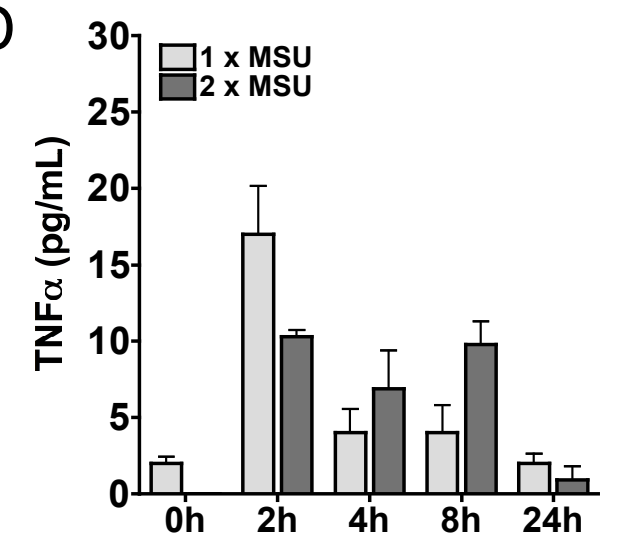

B
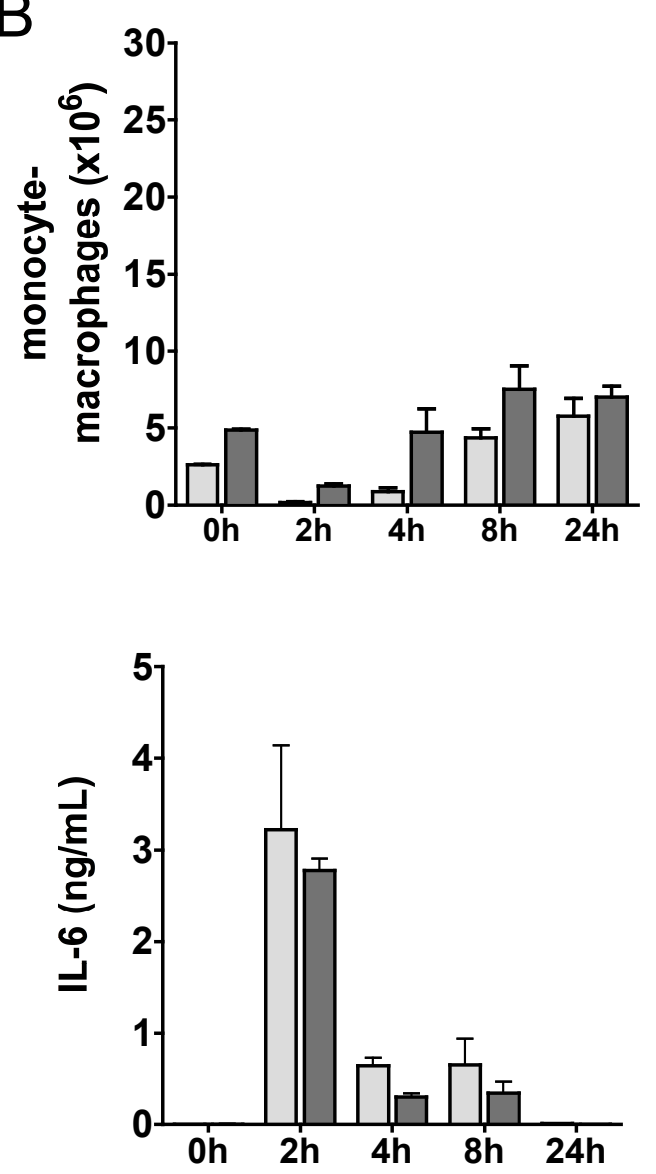

C
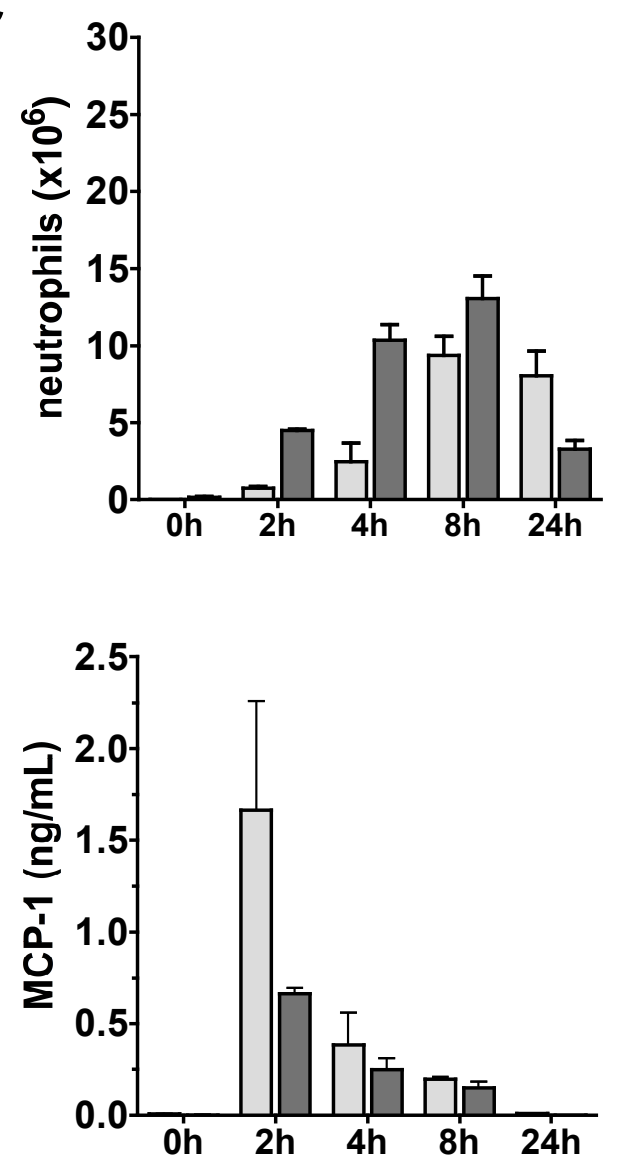
Two days after the first injection of MSU, mice had a similar cell profile in the peritoneum as naïve mice, containing mast cells and lymphocytes but a slightly higher percentage of macrophages in MSU-treated mice $\left(\mathrm{F} 4 / 80^{\text {hi }}\right.$ in naïve mice and F4/80 ${ }^{\text {lo }}$ in mice 2 days after MSU) (Table 6.1 and Fig. 6.10A; 0h). However, total numbers of macrophages were similar in naïve and MSU-treated mice (Fig. 6.10B; 0h). When mice were restimulated with MSU in vivo a more rapid recruitment of both monocytes (Fig. 6.10B) and neutrophils (Fig. 6.10C) was observed. However, the maximum cell numbers were comparable in both groups of mice and peaked at $8 \mathrm{~h}$ after MSU stimulation in both cases (Fig. 6.10A-C). In restimulated mice, macrophages $\left(\mathrm{F} 4 / 80^{\mathrm{lo}}\right)$ also disappeared from the peritoneal lavage fluid, consistent with activation and adhesion to surrounding membranes and tissues following MSU treatment (Fig. 6.10B; 2h).

Increased cellular infiltration did not appear to correlate with higher levels of IL-6, TNF $\alpha$ and MCP-1 production within the first $2 \mathrm{~h}$ of MSU treatment (Fig. 6.10D). In fact, cell infiltration occurred faster despite a slightly lower cytokine production, indicating that $\mathrm{F} 4 / 80^{\mathrm{lo}}$ macrophages may be sensitised to low concentrations of cytokines, or that other factors may be at play. Although recruitment following a second MSU confirmed that monocytes differentiated into pro-inflammatory F4/80 macrophages, it did not explain why recruitment was accelerated. Therefore, to investigate the possible contribution from surrounding tissue, tissue sections were prepared from the peritoneal membranes of naïve mice and from mice after treatment with MSU. Compared to membranes from naïve mice, the epithelial membranes of MSU-treated mice were still swollen and inflamed and had not recovered to a normal background even at $72 \mathrm{~h}$ after MSU treatment (Fig. 6.11). This poor tissue recovery indicated that although neutrophil-based inflammation has resolved by 48 hours, the surrounding non-hemopoietic tissue was still in a state of inflammation several days after the onset of gout inflammation. The swollen epithelium therefore may have presented fewer physical barriers to a second neutrophil migration resulting in a much faster response despite lower cytokine levels in mice restimulated with MSU. 


\section{Naive}

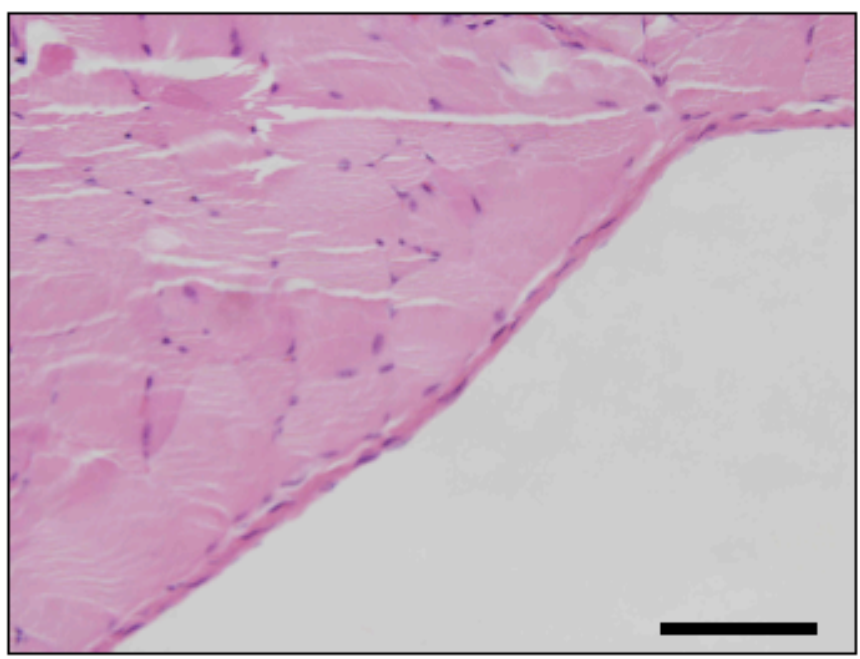

$72 \mathrm{~h}$

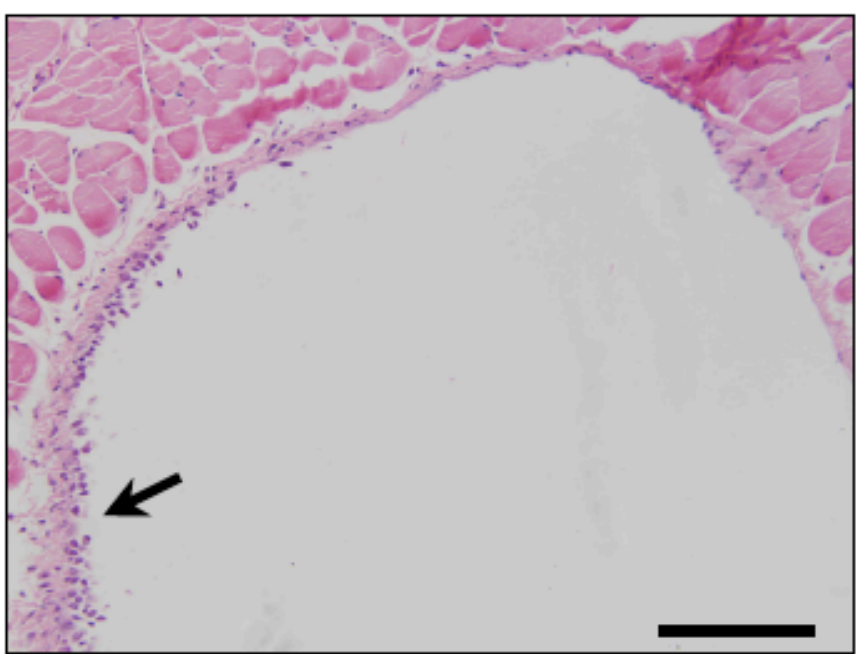

Figure 6.11 Hematoxylin and Eosin staining of peritoneal tissue

Peritoneal tissue from naïve mice and from mice $72 \mathrm{~h}$ after receiving an i.p. injection of MSU were harvested and Hematoxylin and Eosin stained sections prepared. Samples were taken from five separate mice per group. Arrow represents area of inflamed epithelium. Original magnification 40x. Scale bar represents $100 \mu \mathrm{m}$. 


\subsubsection{Investigating phagocytic capacity}

Phagocytosis is an important function of monocytes and macrophages allowing them to take up infectious organisms such as microbes, non-biological matter such as crystals, and dead and dying cells (Leenen and Campbell, 1993). To investigate phagocytic capacity, I examined the expression of a phagocytosis receptor, CD206 (the mannose receptor) as well as the ability of differentiating monocytes to phagocytose fluorescent beads.

\subsubsection{Expression of CD206}

The mannose receptor CD206 is commonly used as a marker of phagocytic capability (Kruskal et al., 1992). To identify potential phagocytic activity of infiltrating monocytes CD206 expression was measured over the MSU-induced acute response in vivo. As shown in Figure 6.12, CD206 did not appear to be expressed on newly recruited monocytes (Fig. 6.12; 4h). However, the $\mathrm{Gr}-1^{+}$ monocytes began to upregulate CD206 around the same time that pro-inflammatory cytokine production ceased and peaked at the point when cell numbers - in particular, neutrophils - began to decline (Fig. 5.3A and Fig. 6.12). It therefore appeared that phagocytic capacity of infiltrating monocytes was optimal during the period of neutrophil clearance indicating that monocyte-macrophages may contribute to the resolution phase of MSU-induced inflammation. Interestingly, after $48 \mathrm{~h}$, corresponding to the period when F4/80 expression began to increase, CD206 expression on monocyte-macrophages downregulated to levels comparable to resident macrophages, providing further evidence that monocytes differentiate into resident-like mature macrophages.

\subsubsection{Functional phagocytic bead assay}

To observe the phagocytic capacity of differentiating monocytes, the uptake of fluorescent beads was used as a direct measurement of phagocytosis. I first sought to optimise the concentration of beads for the effective measurement of phagocytosis. A bead titration was performed using a mixture of cells from the peritoneum on naïve mice, and from mice $8 \mathrm{~h}$ after treatment with MSU. This cell 


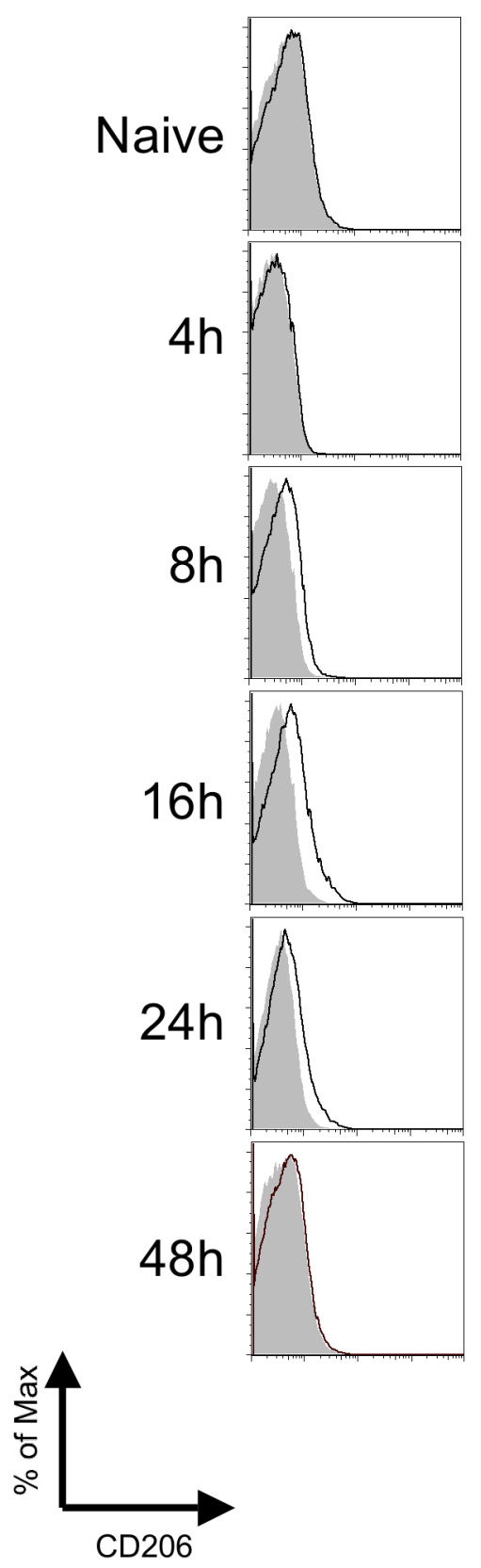

Figure 6.12 Expression of the mannose receptor $\mathrm{CD206}$ on $\mathrm{F4}^{-80^{+}}$cells over the course of an MSU-induced acute inflammatory response.

Mice were treated with an i.p. injection of MSU (3mg MSU, 0.5mL PBS) and over the course of the response peritoneal exudate cells were harvested by lavage $(3 \mathrm{~mL}$ PBS). Harvested cells were prepared and stained for surface markers and analysed by flow cytometry. Monocytes were identified as cells expressing F4/80 and were also identified by Gr-1 and 7/4 where possible. Results are representative of three separate experiments. 
suspension contained a mixture of monocytes, macrophages, neutrophils and lymphocytes. Since the original bead concentration was unknown, the concentration of beads was expressed as a dilution factor of the original stock solution.

Monocyte/macrophages phagocytosed beads after 30 minutes incubation; and this could be visualised either on slides or by flow cytometry (Fig. 6.13A-B). As determined by flow cytometry, the percentage of bead ${ }^{+}$cells formed a sigmoid curve when plotted against bead dilution, with the steepest part of the curve occurring between dilution factors of $10^{-4}$ and $10^{-5}$. Interestingly, not all cells phagocytosed beads, with only $35 \%$ of cells phagocytosing beads even at the highest bead concentration (Fig. 6.13C, dilution factor $5 \times 10^{-2}$ ). A bead dilution factor of $5 \times 10^{-4}$ was chosen to provide greatest sensitivity to changes in bead uptake by monocyte/macrophage populations.

\subsubsection{Phagocytic capacity of differentiating monocytes}

To investigate the phagocytic activity of infiltrating monocytes over time, peritoneal cells were harvested over the course of an in vivo MSU response and then exposed to fluorescent beads for 30 minutes. Peritoneal cells from a naïve mouse, containing mature macrophages, were included as a control. Monocyte/macrophage populations were identified as $\mathrm{F} 4 / 80^{+}$cells, and neutrophils were identified and excluded using their phenotypic expression as $\mathrm{F} 4 / 80^{-}, \mathrm{Gr}-1^{+}$cells.

As shown in Figure $6.14,43 \%$ of peritoneal macrophages phagocytosed beads, and the majority of those macrophages phagocytosed more than one bead as determined by flow cytometry (Figs. 6.14 and 6.15). At 4 hours after gout induction only $6 \%$ of infiltrating monocytes contained phagocytosed beads showing that infiltrating monocytes were only weakly phagocytic upon entry into the inflamed peritoneum (Fig. 6.14). At 8 and 16 hours, infiltrating monocytes had become more phagocytic, with nearly $30 \%$ bead $^{+}$monocytes. This increased again at 24 hours, with nearly $40 \%$ of monocytes/immature macrophages phagocytosing beads. Although the proportion of bead ${ }^{+}$monocytes/immature macrophages at this time point was 


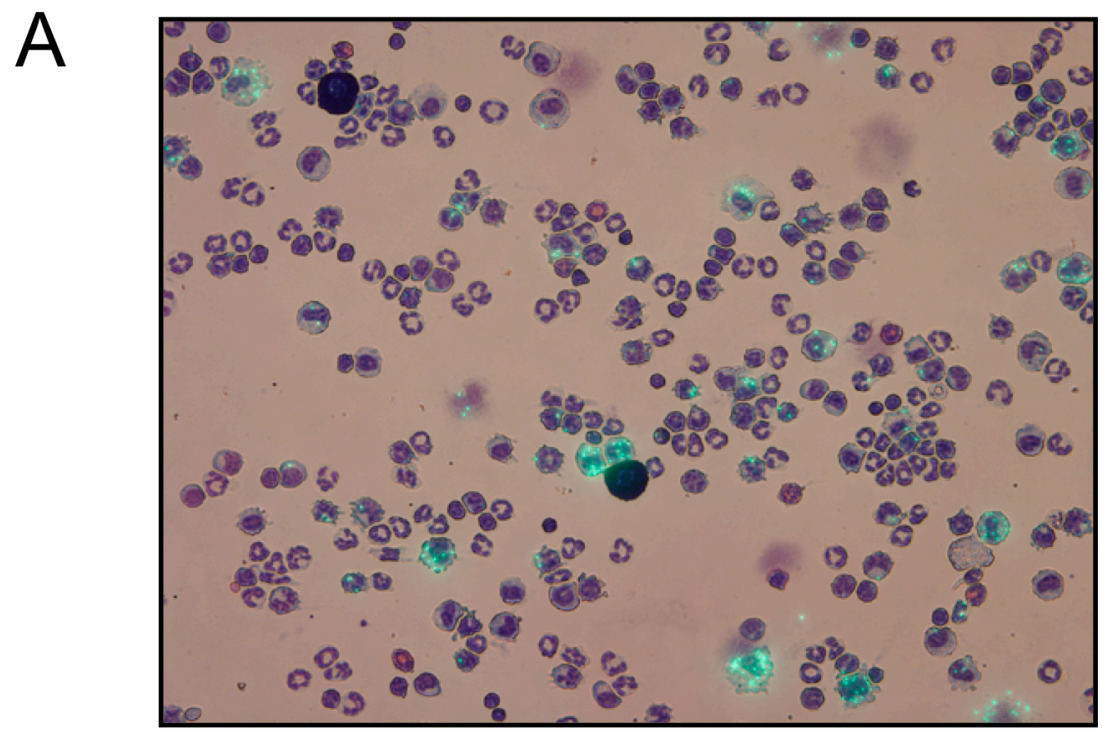

B

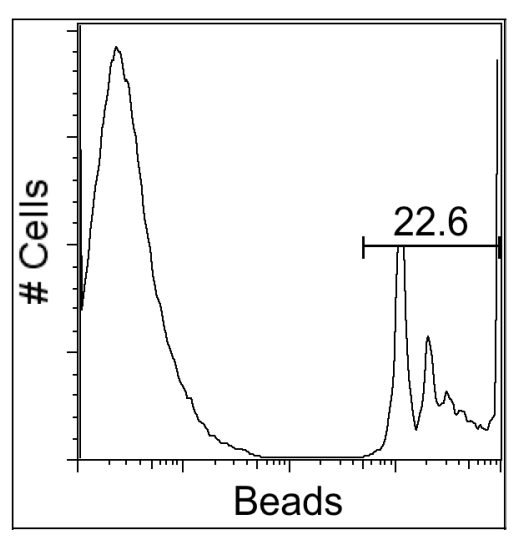

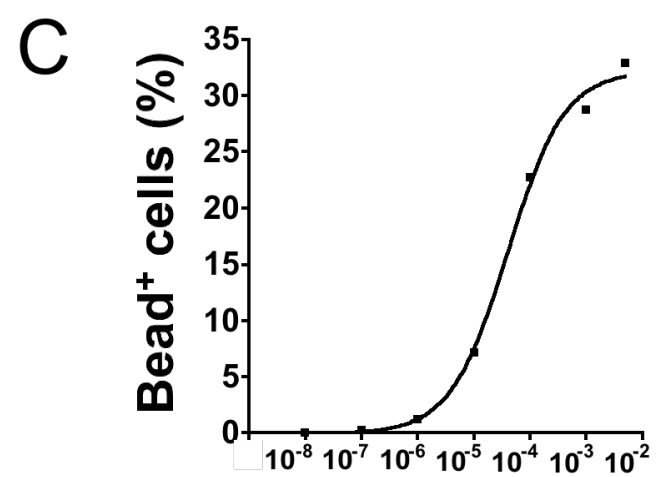

Dilution factor

Figure 6.13 Optimisation of bead concentration for use in the phagocytosis assay

A cell suspension containing mixed samples of peritoneal exudate cells $(1 \mathrm{~mL}, 1 \mathrm{x}$ $10^{6}$ cells) was incubated with various concentrations of fluorescent beads for 30 mins. Excess beads were then washed off and phagocytosis of beads visualised either by cytospin (A) or flow cytometry (B). A dose titration was determined based on flow cytometry data (C). Original magnification 40x. Results are representative of two separate experiments. 

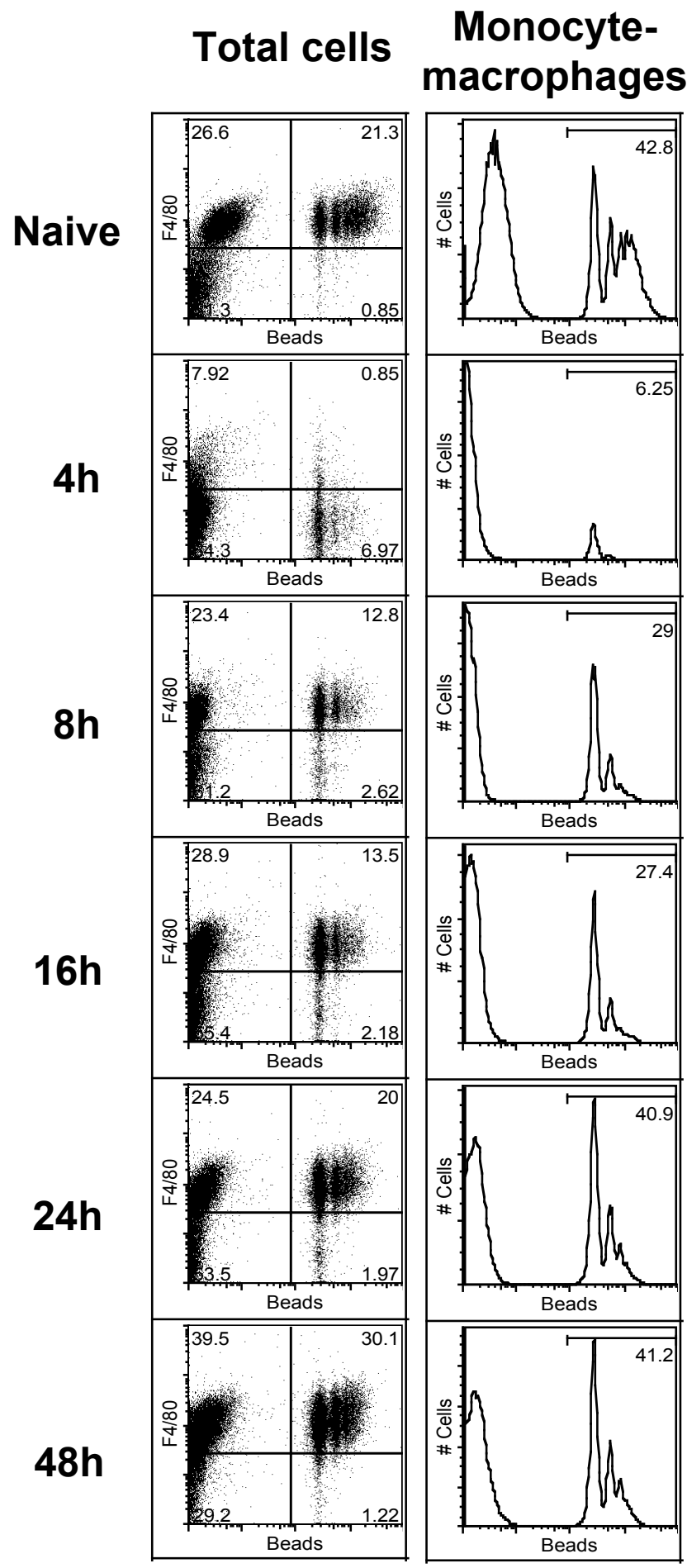

Figure 6.14 Phagocytosis of fluorescent beads by monocyte-macrophages

Mice were treated with an i.p. injection of MSU (3mg MSU, 0.5mL PBS) and over the course of the response peritoneal exudate cells were harvested by lavage $(3 \mathrm{~mL}$ PBS). Harvested, washed peritoneal exudate cells from each time point $(1 \mathrm{~mL}, 1 \mathrm{x}$ $10^{6}$ cells) were incubated with fluorescent beads for 30 mins. Excess beads were washed off and cells were stained with surface markers and analysed by flow cytometry. Results are representative of three separate experiments. 


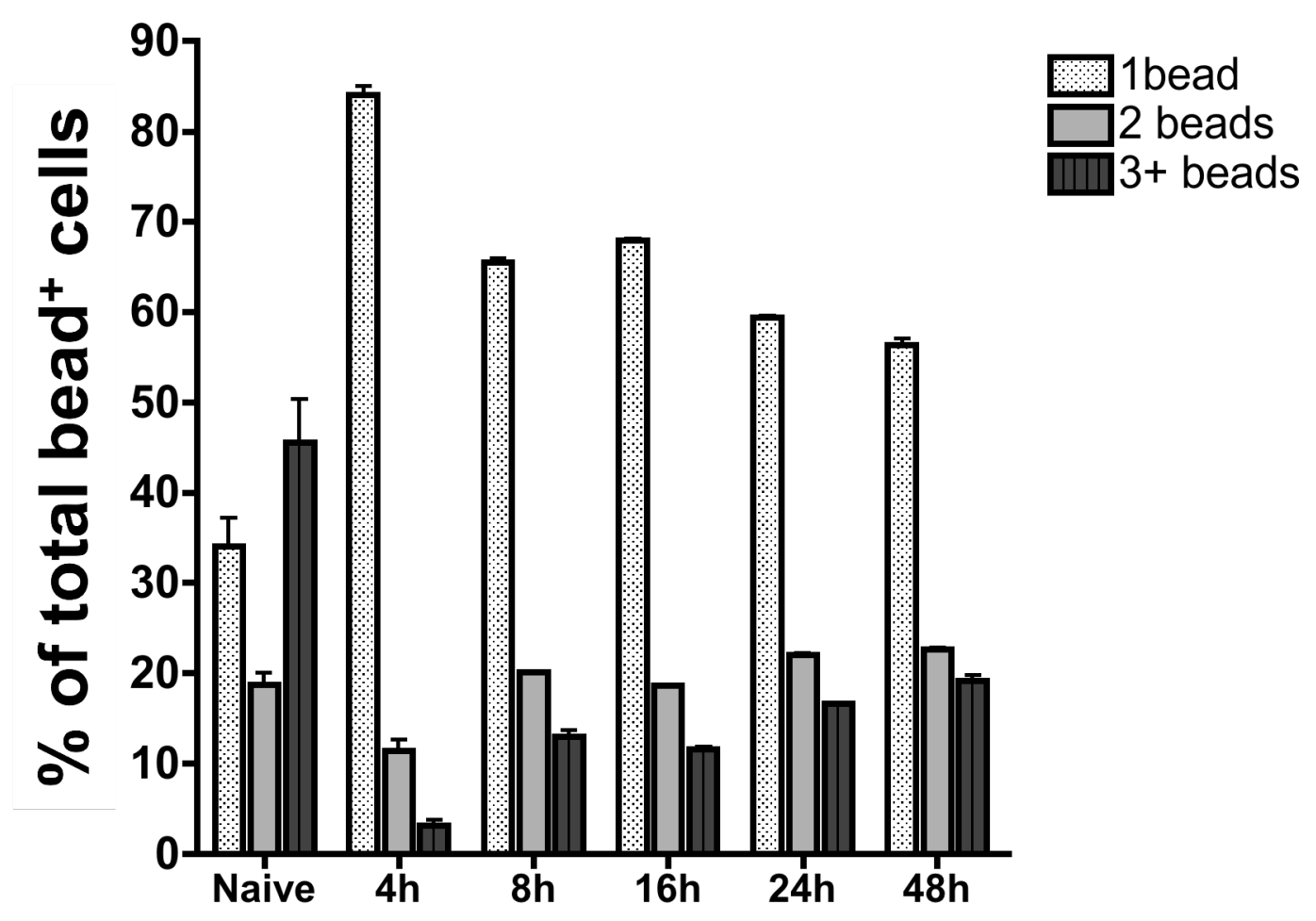

Figure 6.15 Enumeration of beads taken up by monocyte-macrophages

Total F4/80 ${ }^{+}$, bead ${ }^{+}$cells in Figure 6.14 were divided into groups based on whether they had taken up one bead, two beads, or three or more beads using the peaks in flow cytometry histograms. Measurements were performed in duplicate. Values represent mean \pm S.E.M. Results are representative of three separate experiments. 


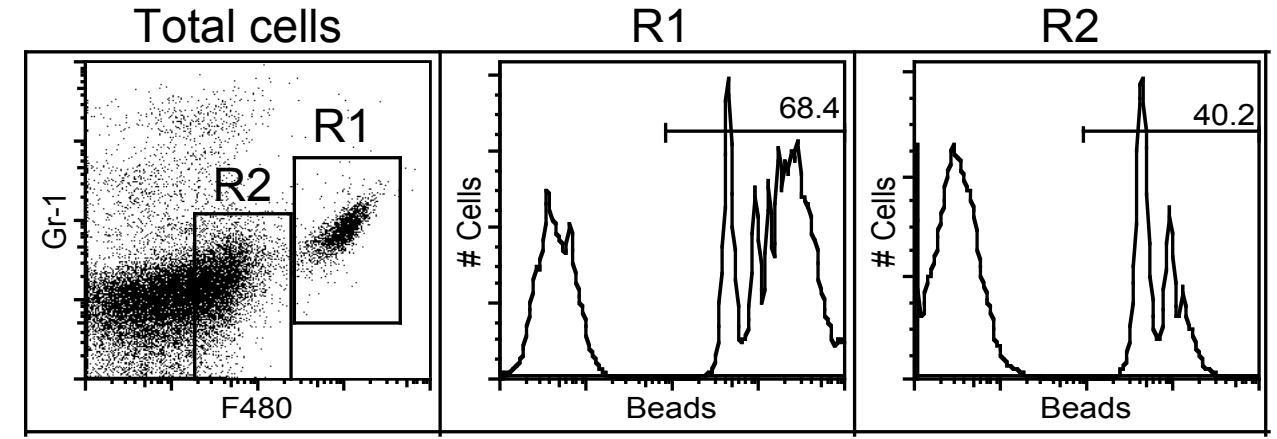

Figure 6.16 Phagocytic capacity of macrophages from mice $72 \mathrm{~h}$ after in vivo

\section{MSU treatment}

Mice were treated with an i.p. injection of MSU (3mg MSU, 0.5mL PBS) and after $72 \mathrm{~h}$ peritoneal exudate cells were harvested by lavage $(3 \mathrm{~mL}$ PBS). Harvested, washed peritoneal exudate cells $\left(1 \mathrm{~mL}, 1 \times 10^{6}\right.$ cells $)$ were incubated with fluorescent beads for 30 mins. Excess beads were washed off and cells were stained with surface markers and analysed by flow cytometry. Results are representative of three separate experiments. 
comparable to that of resident macrophages, resident macrophages exhibited greater phagocytic capacity with $45 \%$ phagocytosing three or more beads compared with $20 \%$ of the immature macrophages. This was consistent with recruited monocytes having an immature phenotype $24 \mathrm{~h}$ after MSU treatment in vivo (Fig. 6.15).

Based on earlier experiments indicating upregulation of F4/80 several days after MSU administration, the phagocytic capacity of both $F 4 / 80^{\text {lo }}$ and F4/80 hi cell populations was examined three days after MSU treatment. Peritoneal cells harvested from mice three days after MSU treatment in vivo contained both F4/80 immature macrophages and a population that had developed into an F4/80 hi phenotype (Fig. 6.16). F4/80 mi macrophages in the population (R1) showed a greater proportion of bead ${ }^{+}$cells as well as a higher uptake of beads per cell than F4/80 macrophages in the same cellular exudate (R2). This indicated that increased phagocytic capacity was associated with an increase in F4/80 expression and maturation.

Collectively these data indicated that when $\mathrm{Gr}-\mathrm{1}^{+}$monocytes were first recruited into the peritoneum, they were not very phagocytic. As they differentiated into immature macrophages they developed an increased phagocytic ability, which increased even further once they differentiated into $\mathrm{F} 4 / 80^{\mathrm{hi}}$ phenotype, more closely resembling a mature resident phenotype.

\subsubsection{Investigating the effect of neutrophil uptake on monocyte responses}

The development of phagocytic capacity of monocytes in vivo, as well as the increased responsiveness of differentiating monocytes to MSU, did not appear to correlate with increased cytokine production in vivo. Immature macrophages had become responsive to MSU by $24 \mathrm{~h}$ after recruitment (Fig. 6.8), but by $24 \mathrm{~h}$ the levels of cytokines found in vivo had returned to normal (Fig. 6.10) while the cell profile returned to levels comparable with naïve controls by $48 \mathrm{~h}$ (Fig. 6.10 and Fig. 5.4). These in vivo data appeared to conflict with my other functional data showing that during the resolution phase of the inflammatory response, the immature macrophage population originating from the infiltrating monocytes shift towards a proinflammatory phenotype (Figs. 6.8 and 6.10). This led to the question as to what was causing a resolution of pro-inflammatory cytokine production. 
Previous research has suggested that the uptake of apoptotic neutrophils by macrophages may be important in the clearance of a gout inflammation response (Rose et al., 2006). Although this research showed prolonged inflammation in the absence of neutrophil uptake, the researchers had used a number of artificial and somewhat irrelevant systems to model resolving gout inflammation including thioglycollate-elicited macrophages and apoptotic thymocytes that contained a mixture of cell types. In addition, they did not explore any differences in the production of pro-inflammatory cytokines from monocyte/macrophages following the uptake of apoptotic cells and subsequent exposure to MSU. I therefore asked the question as to whether differentiating monocytes played a role in the resolution of gout through the phagocytosis of apoptotic neutrophils using monocytes and neutrophils recruited in response to MSU inflammation, thus providing more physiologically relevant observations.

\subsubsection{Monocyte phagocytosis of apoptotic cells}

To gain insight into whether monocyte/macrophages phagocytic capacity might be contributing to the resolution phase of acute gout, a means to measure the phagocytosis of neutrophils by infiltrating immature macrophages was sought.

Neutrophils contain a high level of myeloperoxidase (MPO) within their azurophilic granules that can be detected using the histochemical dye $o$-dianisidine. Using this dye, mononuclear phagocytes that have ingested neutrophils are readily identified by a brown staining within the cytoplasm, corresponding to the presence of MPO. A clear advantage of this detection method is that it allows analysis of the normal inflammatory environment without the need for the uptake of pre-labelled cells.

To investigate the phagocytic capacity of the infiltrating monocytes at different stages of differentiation, peritoneal cells were harvested at different time points after MSU treatment and stained for MPO and either immediately or after an in vitro culture for 18 hours. Immediate staining of the peritoneal cells provided an "in situ snapshot" of the phagocytosis that was occurring in vivo; while staining following in vitro culture was carried out to determine the capacity of monocyte-macrophages to take up neutrophils. Monocyte-macrophages were scored either MPO low or high. 
Low MPO staining macrophages contained only a few particles of MPO staining, while high MPO staining cells either contained extensive MPO staining through the cytoplasm, or contained phagocytosed neutrophils within the cytoplasm.

At 4 hours after MSU administration there were very few monocytes present, as had been shown in earlier investigations (Fig. 6.17; 4h and Fig 5.3B; 4h). Peritoneal exudate cells were comprised mostly of neutrophils, and these neutrophils stained positively for peroxidase. After in vitro incubation very few monocytes stained positively for peroxidase consistent with the earlier bead data that these monocytes were not phagocytic at this time point (Fig. 6.17; 4h and Fig. 6.18A and C). $\mathrm{MPO}^{+}$ cells began to appear 8 hours after MSU treatment when stained immediately after harvest (Fig. 6.18A). The percentage of $\mathrm{MPO}^{+}$monocyte-macrophages continued to increase up to 24 hours. When $\mathrm{MPO}^{+}$monocyte-macrophages were separated as high and low staining, only a small proportion of cells were MPO ${ }^{\text {hi }}$, but this percentage steadily increased up to 24 hours.

After in vitro incubation, monocyte-macrophages from 8 to $24 \mathrm{~h}$ mice not only showed an increase in the percentage of $\mathrm{MPO}^{+}$cells, but also showed a large increase in high MPO $^{\text {hi }}$ monocyte-macrophages, indicating the uptake of greater numbers of neutrophils per mononuclear phagocyte (Figs. 6.17 and 6.18C and D). These results showed that monocytes increased their uptake of apoptotic neutrophils in vivo over time, and have a high capacity for neutrophil uptake from eight hours on. In cells from mice treated with MSU for $48 \mathrm{~h}$, there was very little peroxidase staining in macrophages and very few neutrophils consistent with resolution of the acute inflammatory response and neutrophil clearance in vivo.

\subsubsection{The uptake of apoptotic neutrophils leads to macrophage quiescence}

The uptake of apoptotic neutrophils has been shown in other systems to switch mononuclear cells from pro- to anti-inflammatory, leading to the production of cytokines involved in resolution including TGF $\beta$ (Fadok et al., 1998). The uptake of apoptotic neutrophils in the in vivo gout model may therefore also be exerting an anti-inflammatory influence contributing to resolution of the inflammatory environment. 


\section{Figure 6.17 Peroxidase staining of peritoneal exudate cells}

Mice were treated with an i.p. injection of MSU (3mg MSU, 0.5mL PBS) and over the course of the response, peritoneal exudate cells were harvested by lavage $(3 \mathrm{~mL}$ PBS). Cytospin slides of the cells were prepared either immediately or after incubation for $18 \mathrm{~h}$ in vitro, stained with myeloperoxidase stain and counterstained with Diff-Quik. Black arrows represent cells containing phagocytosed neutrophils. Original magnification 40x. Results are representative of three separate experiments. 


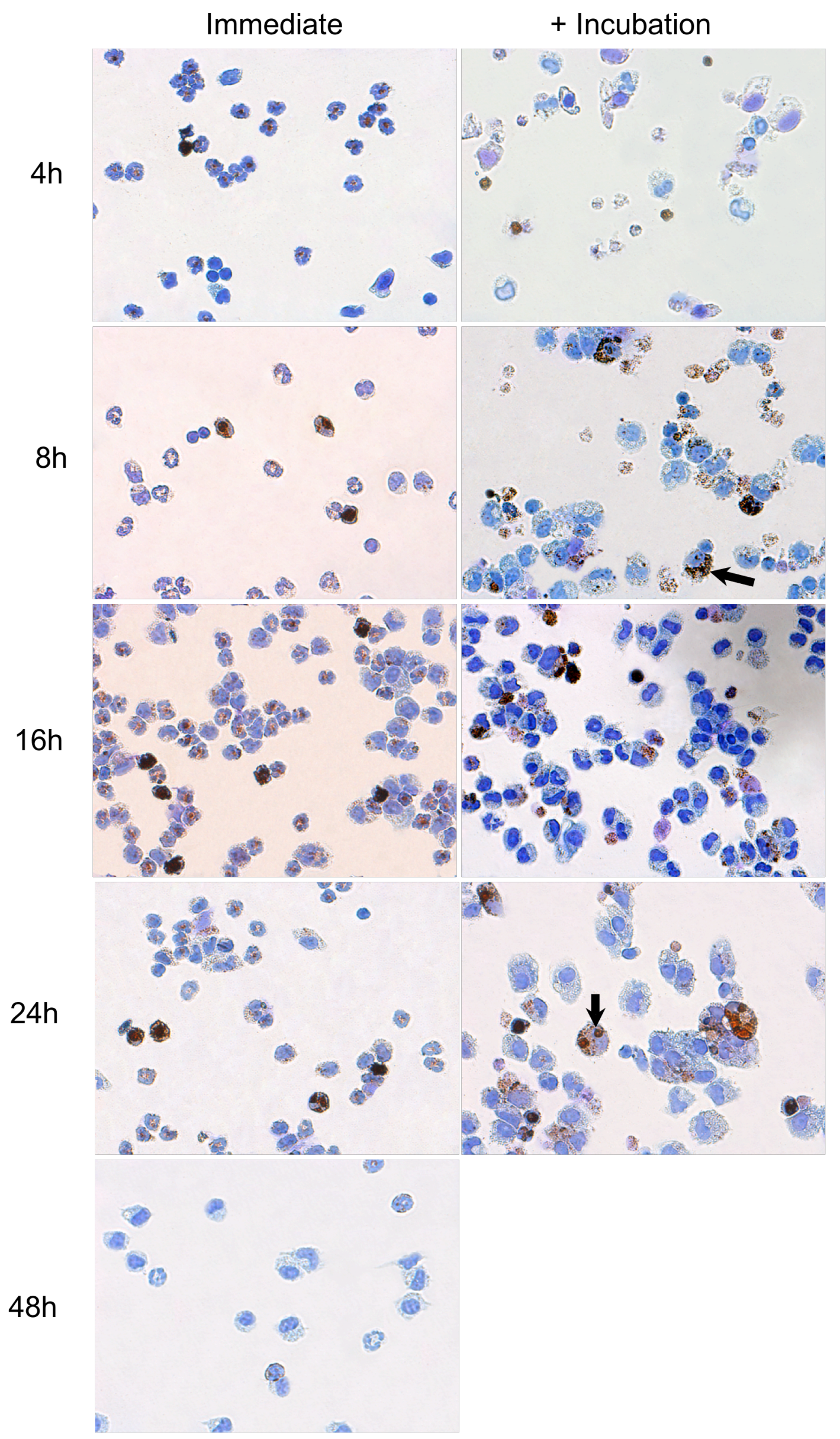


A

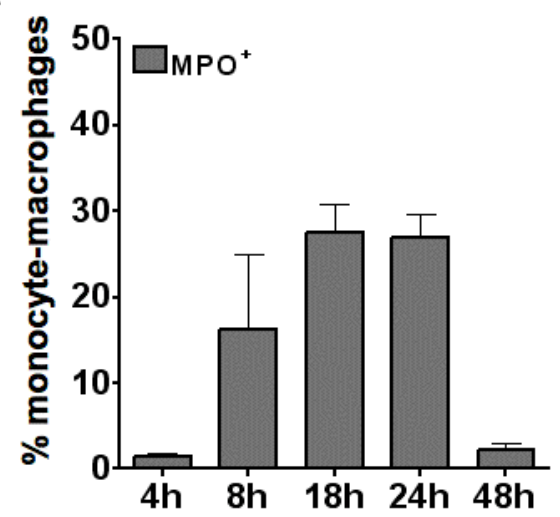

C

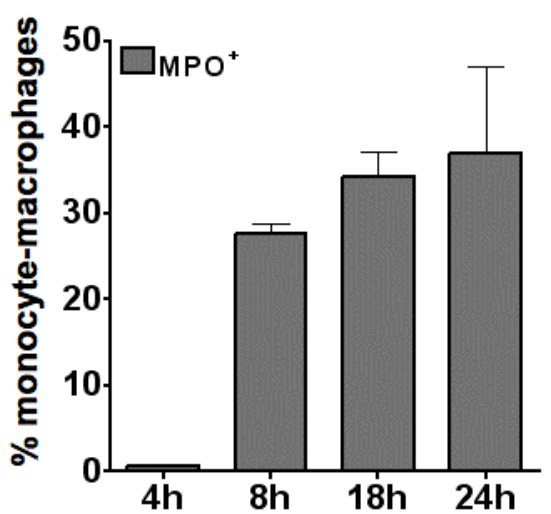

B

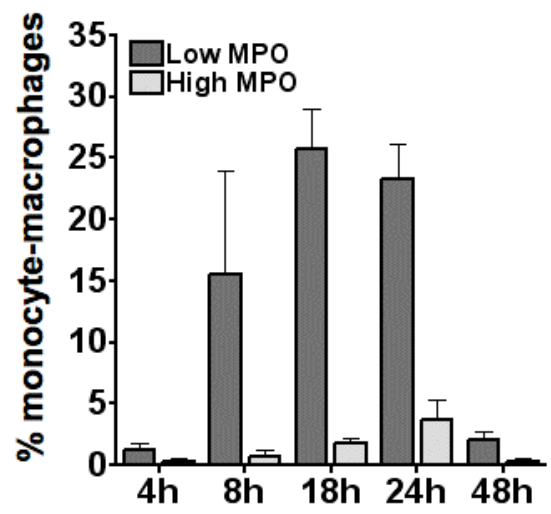

D

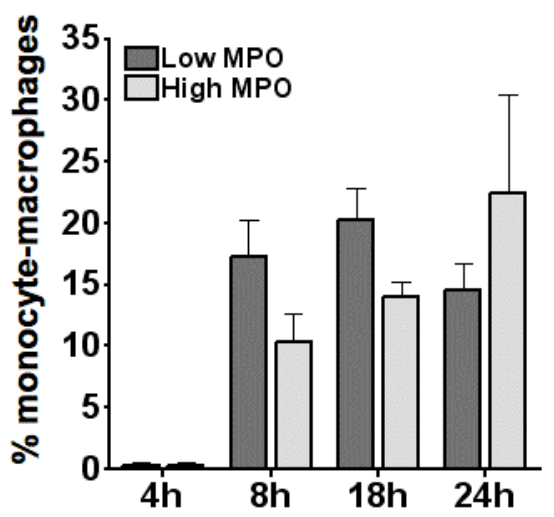

Figure 6.18 Percentage of myeloperoxidase positive $\left(\mathrm{MPO}^{+}\right)$monocytemacrophages in peritoneal exudate cells

The percentages of monocyte-macrophages from Figure 6.17 containing MPO were determined by microscopic examination. The percentage of $\mathrm{MPO}^{+}$monocytemacrophages was determined immediately after harvesting either as total $\mathrm{MPO}^{+}$cells (A) or divided into high and low MPO staining (B). Alternatively, the percentage of $\mathrm{MPO}^{+}$monocye-macrophages was determined after in vitro incubation for a further 18h. The percentage of $\mathrm{MPO}^{+}$monocyte-macrophages was again determined immediately after harvesting either as total $\mathrm{MPO}^{+}$cells $(\mathbf{C})$ or divided into high and low MPO staining (D). Measurements were performed in triplicate. Values represent mean \pm S.E.M. Results are representative of three separate experiments. 
To determine if the uptake of neutrophils initiated a more anti-inflammatory monocyte-macrophage cell-type in gout, peritoneal cells were harvested from mice treated with MSU for $18 \mathrm{~h}$ (a time point corresponding to the resolution phase of inflammation). Peritoneal leukocytes were then either stimulated immediately with MSU or incubated ex vivo for $4 \mathrm{~h}$ and $22 \mathrm{~h}$ (to allow neutrophil uptake) and then stimulated with MSU. MPO staining of the immature macrophages showed that the percentage of $\mathrm{MPO}^{+}$macrophages increased following $4 \mathrm{~h}$ and $22 \mathrm{~h}$ incubation ex vivo indicating an increased uptake of apoptotic neutrophils (Fig. 6.19A). If neutrophil uptake were exerting a resolving effect on macrophages, then a lower level of MSUstimulated cytokine production would be expected.

Out of all of the cytokines measured (TNF $\alpha$, IL-6, MCP-1, IL-1 $\beta$ ) only IL-1 $\beta$ was produced following exposure to MSU, indicating that these immature macrophages were only just acquiring responsiveness to MSU consistent with previous data that showed monocytes only became responsive to MSU between $16 \mathrm{~h}$ and $24 \mathrm{~h}$ (Fig. 6.8). In the case of IL-1 $\beta$, there was a significant reduction in the responsiveness of the harvested cells to MSU stimulation, and this reduction was enhanced with a longer incubation with apoptotic neutrophils (Fig. 6.19B). This phenomenon was also observed when the cells were stimulated with LPS, a result consistent with data previously reported in the literature (Fadok et al., 1998). These results show that the uptake of apoptotic neutrophils by immature macrophages was associated with a decreased pro-inflammatory response to MSU and strongly implicates the uptake of neutrophils by immature macrophages as a potential shut-down mechanism for acute gouty inflammation.

\subsubsection{Monocyte-macrophages in the resolution of inflammation}

TGF $\beta$ is thought to play a central role in the resolution of gout inflammation. It has been proposed that macrophages generate TGF $\beta$ in the resolution of gout inflammation, however this has not been shown in vivo. Meanwhile, other studies have shown the production of TGF $\beta$ by macrophages as a result of neutrophil uptake in vitro. 

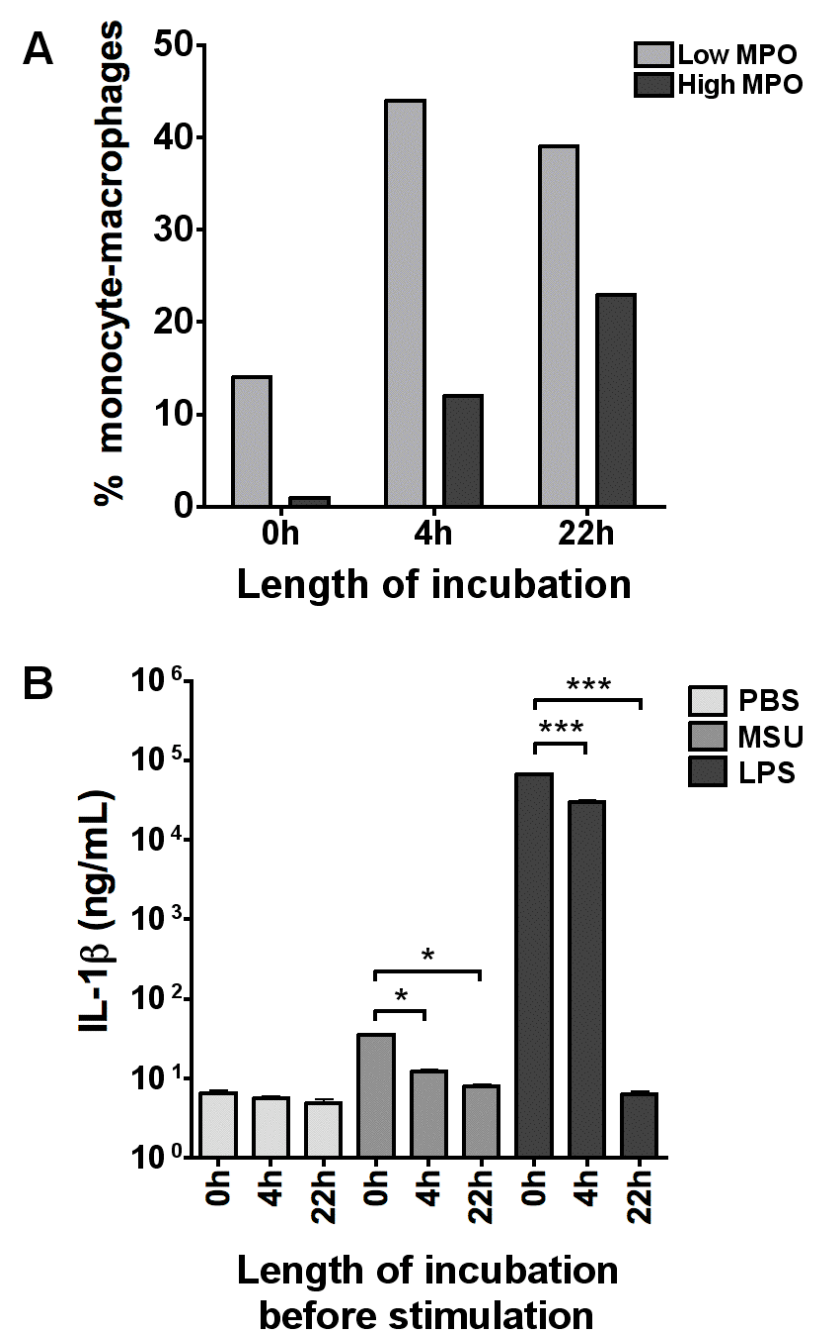

Figure 6.19 IL-1 $\beta$ production from peritoneal leukocytes following neutrophil uptake

Mice were treated with an i.p. injection of MSU (3mg MSU, 0.5mL PBS) and the peritoneal cells harvested by lavage after 18h. A. Cells were then stained for MPO either immediately, or after $4 \mathrm{~h}$ or $22 \mathrm{~h}$ incubation ex vivo. The percentage of $\mathrm{MPO}^{+}$ staining monocyte-macrophages was determined by microscopic examination. B. Cells $\left(1 \times 10^{6} / \mathrm{mL}\right)$ were then stimulated with MSU $(500 \mu \mathrm{g} / \mathrm{mL})$ or LPS $(100 \mathrm{ng} / \mathrm{mL})$ either immediately, or after ex vivo incubation for either $4 \mathrm{~h}$ or $22 \mathrm{~h}$. Supernatants were collected after $8 \mathrm{~h}$ and the level of IL-1 $\beta$ determined by ELISA. $*=P<0.05$ and $* * *=P<0.001$ as determined by one way ANOVA and Tukey multiple comparisons test. Results are representative of three separate experiments. 
To determine whether TGF $\beta$ was exerting a role in the mouse model, lavage fluids were harvested from mice at different times over the MSU inflammatory response and were assayed for levels of TGF $\beta$. A total of $3 \mathrm{~mL}$ of PBS was used to lavage the peritoneum, which meant that TGF $\beta$ was necessarily diluted. The detectable levels of TGF $\beta$ were very low, the highest mean value being only $4 \rho g / \mathrm{mL}$ (Fig. 6.20). Following MSU treatment there was a reproducible trend towards a decrease in TGF $\beta$, yet the standard errors of the values were too high to show any statistical significance. Another group (Rose et al., 2006) has also observed this same trend, indicating that there is a background level of TGF $\beta$ that decreases once MSU is administered. This background TGF $\beta$ may exert a minor suppression on tissue macrophages that is released after MSU treatment. However, it is also possible that TGF $\beta$ may not even participate in general suppression. Interestingly, TGF $\beta$ levels did not return to homeostatic levels over the time resolution was observed in vivo (Fig. 6.20; 16-48h). In addition, IL-10, an alternative suppressive mediator, was undetectable in the lavage fluid over the whole timecourse indicating that IL-10 does not play a role in gout resolution (data not shown). In any case, the low levels of soluble TGF $\beta$ in vivo precluded the measurement of TGF $\beta$ as a direct measure of macrophages functioning as anti-inflammatory cells during resolution.

\subsection{Discussion}

This research provides the first comprehensive phenotyping and profiling of monocytes recruited in MSU-induced gout inflammation. This characterisation was performed using changes in morphology, marker expression, and functionality. These changes are summarised in Figure 6.21.

Monocytes that were recruited in response to MSU were of the "inflammatory" subtype and were macrophage precursors. This study showed that as monocytes differentiated into macrophages, they went through at least two stages of differentiation, determinable by changes in marker expression. In the first stage, monocytes differentiated into immature macrophages. This was observed by a diminishing expression of myeloid differentiation antigens (Gr-1, 7/4) as well as an 


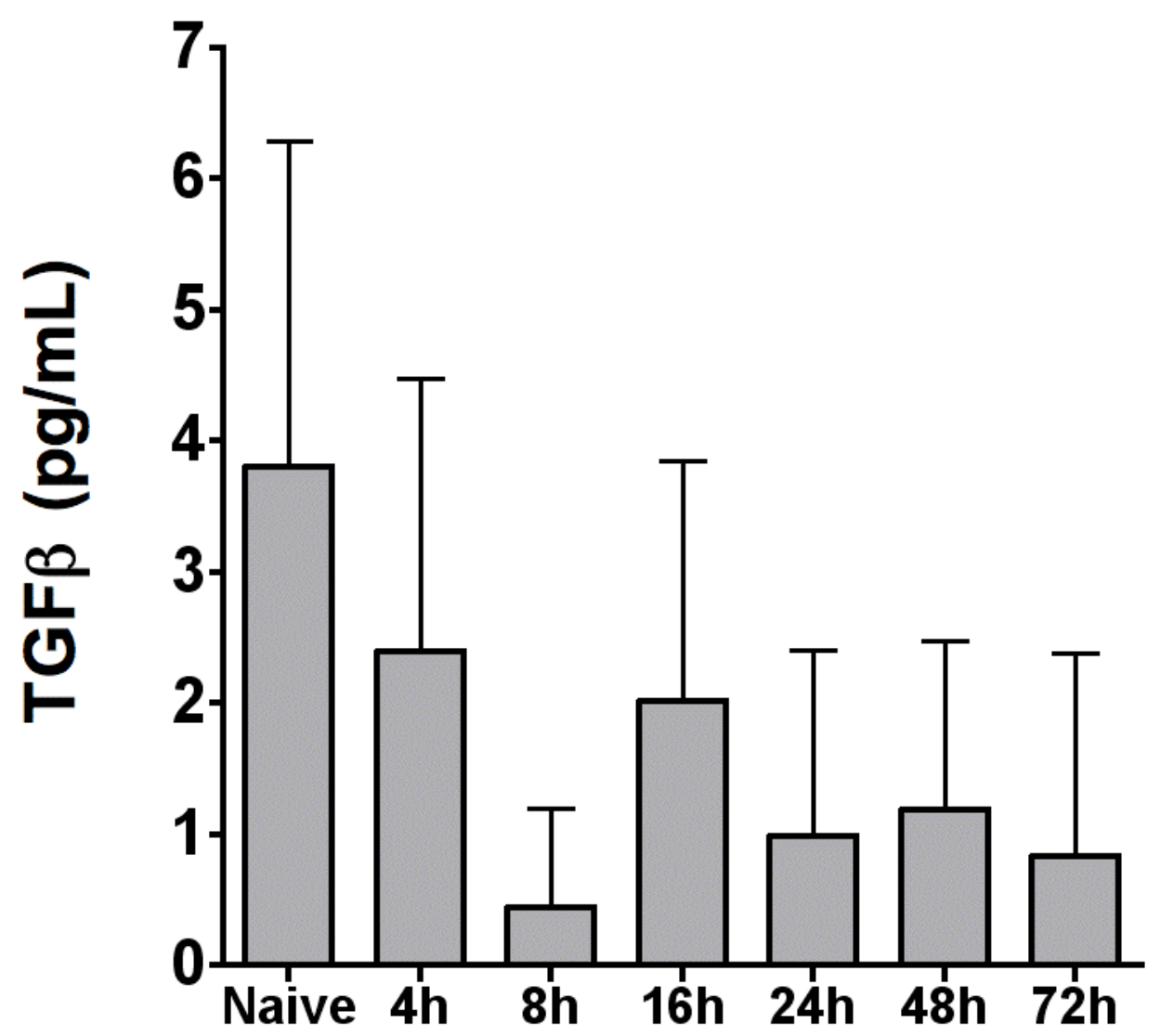

Figure 6.20 TGF $\beta$ levels in the peritoneum over the course of an MSU-induced acute inflammatory response.

Mice were treated with an i.p. injection of MSU (3mg MSU, 0.5mL PBS) and over the course of the response the peritoneum was rinsed with lavage fluid (3mL PBS) and harvested. Levels of TGF $\beta$ were determined by ELISA. Measurements were performed in triplicate. Values represent mean \pm S.E.M. Results are representative of three separate experiments. 


\begin{tabular}{|l|l|}
\hline $\begin{array}{l}\text { Recruited } \\
\text { Monocyte }\end{array}$ \\
\hline
\end{tabular}
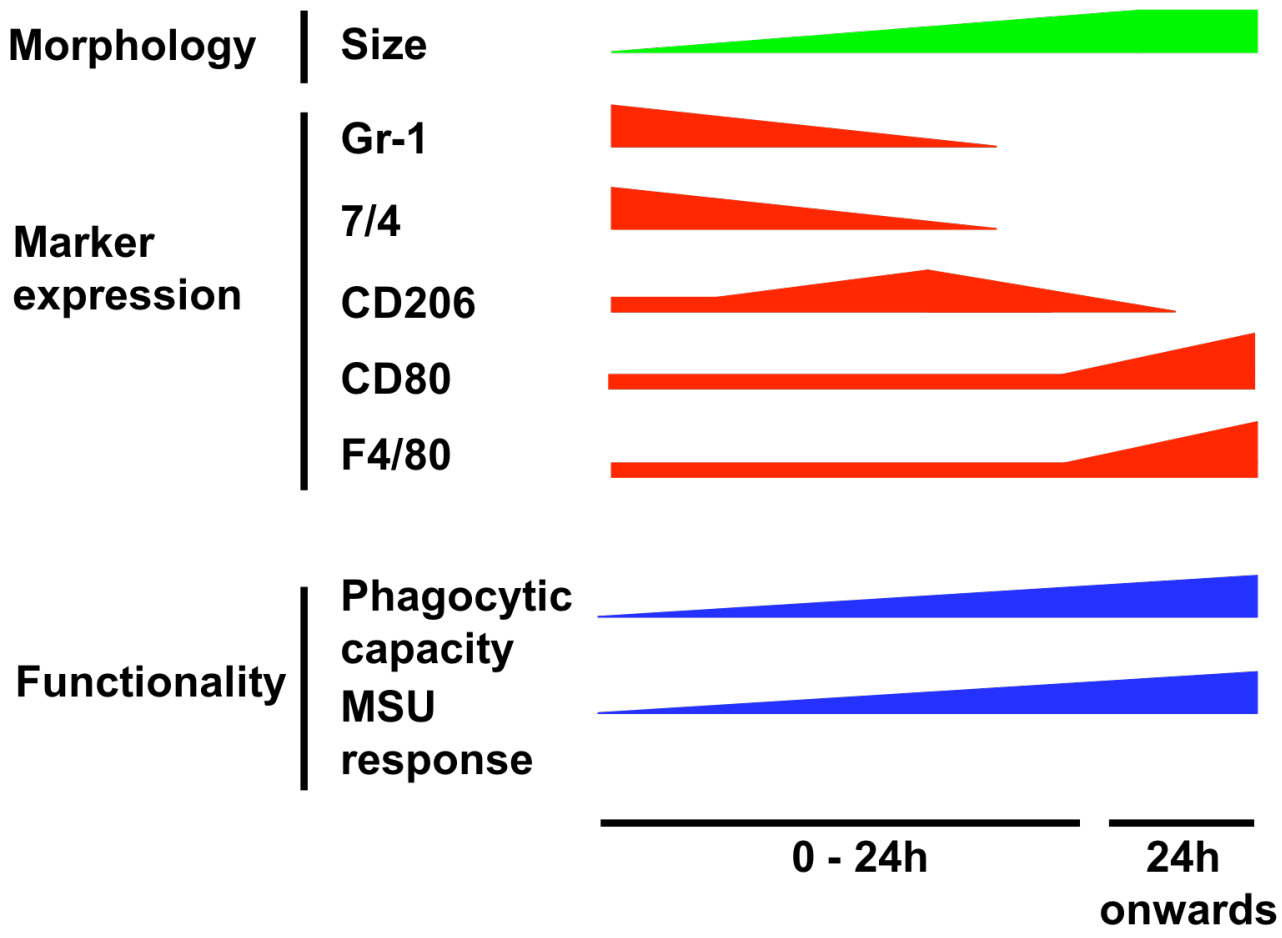

Figure 6.21 Summary of the phenotypic and functional changes during the differentiation of monocytes to macrophages over the course of MSU-induced inflammation. 
increase in size. In the second stage, immature macrophages further differentiated into mature, resident-like macrophages, associated with increased expression of F4/80.

The stages of monocyte differentiation into immature, and then to mature macrophages were also associated with changes in functional capacity. When monocytes first entered the peritoneum they were not responsive to MSU and had poor phagocytic ability as shown by the phagocytosis of beads and of apoptotic neutrophils. As they differentiated into immature macrophages they showed an increasing ability for phagocytosis from approximately $8 \mathrm{~h}$ onwards. However, further increases in phagocytic capacity and cytokine production potential occurred from $24 \mathrm{~h}$ onwards as immature macrophages differentiated further into mature macrophages. Therefore, the differentiation of monocytes into mature macrophages was associated with progressive increases in phagocytic ability, and proinflammatory cytokine responses to MSU.

The mannose receptor CD206 was transiently upregulated on differentiating monocytes indicating that phagocytic ability in terms of sensing mannosylated cell wall components reached an optimum, and then waned. Yet phagocytosis of beads and cells demonstrated that monocytes that had differentiated into macrophages increased in the ability to phagocytose. These data show that the use of CD206 is not so much a marker of phagocytosis, rather, it may be a hardwired mechanism whereby monocytes express CD206 as a surveillance mechanism to detect possible bacterial or other pathogenic infection. Consequently, CD206 may have been downregulated in the absence of this stimulus (as MSU is a sterile inflammation). It would be interesting to determine whether transient CD206 corresponds to some other important process contributing to the MSU response as CD206 is also known to be associated with type II macrophages, which can suppress type I inflammation (Stein et al., 1992, Gordon, 2003).

Where the function of CD206 is known, and so a possible role for CD206 regulation suggested, other differentiation antigens such as F4/80 and 7/4 do not have a current known function. The fact that these markers are regulated on differentiating monocyte/macrophages suggests that these molecules play an important role in 
cellular functioning, yet the actual significance of these molecules has yet to be seen. For instance, despite F4/80 being a key marker of mononuclear phagocytes in mice, eosinophils are the only known human leukocyte to express an F4/80 ortholog (Hamann et al., 2007). Whether this indicates that F4/80 is dispensable for inflammatory responses, or translates into an important functional difference between mouse and human mononuclear phagocytes is currently speculative. Until the function of these molecules is known, it is difficult to hypothesis what their role would be in responses to MSU or in inflammation at large. Nevertheless, they remain very useful tools for tracking differentiation states in mouse monocyte/macrophages.

The differentiation of monocytes into macrophages that were pro-inflammatory rather than anti-inflammatory was not only confirmed in vitro but also in vivo in mice that had received a second dose of MSU at $48 \mathrm{~h}$ after an initial MSU response. Mice that received a second dose of MSU displayed an inflammatory profile that was similar to the mice that had received only one. These results contrast previous hypotheses (discussed in Chapter 5) that macrophages mount an anti-inflammatory response when stimulated with MSU (Yagnik et al., 2004).

There are key differences in my experimental approach that may account for the differences observed. The key difference is that most of their work was performed in vitro using either purified blood monocytes or mixed exudate cells from human blisters recruited from a stimulus that was not MSU (they used cantharidin). My results were based on cells recruited using MSU as the stimulus in vivo, and so I have used and observed cells that were directly relevant to MSU-induced inflammation, thus representing the gouty condition more faithfully. The cells in my study were not only recruited by the appropriate stimulus, but were exposed to conditions in the local environment in vivo that allowed the appropriate phenotypic and functional changes to be undertaken. It can be argued that some of the differences observed may be partly explained by intrinsic differences between mouse and human cells, and it would be difficult to determine the extent of these differences. However, the use of a mouse model clearly offers some advantages over the use of the blister technique alone for which it is difficult to create the appropriate conditions that reflect the in vivo system and provide enough cells to 
carry out an extensive profiling to determine which mononuclear phagocyte type is actually being studied.

An additional advantage of in vivo models is that they allow for extensive profiling of the inflammatory response so that the correct time points corresponding to the pro-inflammatory and resolution phases of the response can be identified. In the skin blister study (Yagnik et al., 2004), two time points were arbitrarily chosen to represent cells from the pro-inflammatory (16h) and resolution phases (48h). However, in the mouse model, cells recruited in response to MSU were in the beginning stages of a resolving environment after 16 hours, with pro-inflammatory cytokines no longer elevated and cell numbers beginning to decline. Therefore without profiling of the cells and inflammatory mediators in response to blister formation it is impossible to know whether the 16 hour time point chosen by the researchers actually corresponded to the inflammatory phase. One way to improve the chances of choosing the correct time points and conditions in human studies is to use in vivo models to design better human experiments. Since human studies are difficult to perform and reproduce given that subjects are not as easily available as in in vivo studies, in vivo models could be used to suggest appropriate time points and conditions to increase the likelihood that the conditions and time points chosen in human studies are more likely to have successful and relevant outcomes.

The experiment exposing mice to a second MSU stimulation in vivo partially addressed an additional issue: that when monocytes developed the capacity to produce pro-inflammatory cytokines in vivo, there are paradoxically no measurable levels of cytokines produced in the peritoneum despite the presence of MSU crystals in the peritoneum for more than one week after injection (observational information from W. Martin). These observations point to the notion that after initial recognition, MSU crystals in some way become unavailable and therefore unable to induce further inflammation such that resident macrophages that come into early contact with MSU are stimulated, but MSU crystals either become coated in proteins or ingested into cells where they are effectively walled off from further interaction with infiltrating cells. In fact, it may be that the lack of an additional stimulus in the peritoneum when monocytes finally develop the capacity to produce pro- 
inflammatory cytokines to MSU prevents an on-going response allowing spontaneous resolution.

Resolution of both cytokine levels and neutrophil numbers was well underway by the time monocytes developed a pro-inflammatory phenotype at $24 \mathrm{~h}$. Therefore, factors other than non-responsiveness to MSU must be at play during the resolution phase. Here I have shown that the uptake of apoptotic neutrophils was associated with a decreased responsiveness to both MSU and LPS after as little as four hours' culture with neutrophils. This meant that the uptake of apoptotic neutrophils exerted a suppressive influence on MSU-responsive macrophages and this process may serve as an active "shut down" mechanism in gout. Although monocytes and immature macrophages themselves did not actively contribute to the generation of proinflammatory cytokines and cell recruitment, the phagocytosis of apoptotic neutrophils may have shut down responses of any cytokine-producing macrophages that may be present.

Differentiating monocytes showed evidence of neutrophil uptake as soon as $8 \mathrm{~h}$ after recruitment, as determined my MPO staining, which increased over time until resolution was virtually complete at $48 \mathrm{~h}$. This meant that as soon as monocytes developed phagocytic capability (which occurred from $8 \mathrm{~h}$ onwards) they became sensitive to the presence of apoptotic neutrophils. Hence, resolution may simply be an interplay between an evolving monocyte phagocytic function and an emerging neutrophil senescence in the absence of additional stimuli. Although TGF $\beta$ has been thought to be involved in the resolution of gouty inflammation, either by being generated following neutrophil uptake or by other means, I was unable to detect a restoration in the levels of TGF $\beta$. This did not preclude the role of TGF $\beta$ in gout resolution; however, other methods such as quantitative PCR may need to be used in future studies to measure TGF $\beta$ in a meaningful way. However, the lack of measurable soluble TGF $\beta$ supports the idea that factors other than TGF $\beta$ were at play during resolution. Although my experiments did not prove that the uptake of neutrophils was the prime cause of the shut down in cytokine production, it provided a strong indication that this line of research warrants further investigation. 
In summary, these data indicate that monocytes recruited in response to MSU do not produce inflammatory cytokines in response to MSU and are poorly phagocytic within the first few hours of recruitment. Over the course of a few hours, these monocytes differentiate into immature macrophages as observed by a loss in Gr-1 and 7/4 expression, an increase in phagocytic capacity, and an increasing ability to produce pro-inflammatory cytokines in response to MSU. Despite developing the ability to produce pro-inflammatory cytokines to MSU, immature macrophages do not produce these cytokines in vivo; instead, resolution of inflammation is observed. Although this may be partly due to a lack of accessibility of MSU crystals in vivo, the uptake of phagocytic neutrophils in vivo may also inhibit the ability of immature macrophages to respond to MSU, thus leading to resolution. After several days immature macrophages differentiate into mature macrophages, replenishing the local resident macrophage population. 


\section{Chapter 7}

General Discussion 


\section{Chapter 7 \\ General Discussion}

Innate immune cells have been recognised as important in acute gout for several decades. As such, there have been many studies that have explored the involvement of neutrophils, monocytes, macrophages, and even endothelial cells in the acute gout response. Here, this thesis offers a deeper insight into the involvement of each of these cell types in gout. The use of a combination of in vitro, in vivo and ex vivo techniques provided a means whereby these cells could be studied both as purified populations, and as cells comprising one of a number of cell types representative of the mixed cellular conditions found during an acute gout attack in vivo. Therefore, in addition to dissecting the contributions of individual cell types to acute gout, interactions between these cell types in the disease context could also be explored. Collectively these studies provide a bigger picture of the cellular events surrounding acute gout inflammation.

\subsection{Investigating mononuclear phagocyte function using in vivo models}

To date, most research into the function of mononuclear phagocytes has been performed in vitro using cells derived from human blood monocytes. Although informative, this approach has several limitations that have been overcome by using in vivo mouse models in this study.

Monocytes and macrophages by their very nature are cells with high plasticity that are influenced by their surrounding environment. In vitro studies are therefore limited in their ability to simulate faithfully the factors present in the in vivo environment affecting mononuclear phagocyte function, response, and differentiation. In the first instance, in vivo models allow the correct stimulus to be used to set up the appropriate inflammatory conditions. In vivo models also mimic the phenotypic adjustments that occur when mononuclear cells are exposed to recruiting cytokines, move through an endothelial layer, infiltrate into the site of inflammation, and are exposed to the factors and tissues that influence their differentiation. Since cellular functions are likely to change as monocytes differentiate, extensive time courses are required so that changes are not overlooked 
simply because an insufficient number of time points were observed. As there are no human models available to observe gouty inflammation in a routine and controlled way, mouse models provide a useful tool to allow all of these phenotypic changes to be measured.

The use of in vivo models and primary cells revealed some unexpected findings that uncover important aspects of gout inflammation that contradict previously held ideas about the progression of gout inflammation, particularly around mononuclear phagocytes.

\subsection{Macrophages and the induction of gout inflammation}

The results of Chapter 5 demonstrated that macrophages are an important source of cytokines in gouty arthritis. In particular, resident macrophages were a source of IL$1 \beta$, which is pivotal in the recruitment of neutrophils in gout. This was most clearly demonstrated in macrophage-depleted mice, which showed a reduction of IL-1 $\beta$ as well as a corresponding reduction in neutrophil recruitment compared to the responses of naive mice treated with MSU. In addition to IL-1 $\beta$, macrophages were also shown to be a source of IL-6, which also showed reduced levels in macrophagedepleted mice.

Although macrophages are an important source of pro-inflammatory cytokines, they are not an exclusive source of MSU-induced cytokine production in vivo. Despite resident macrophages producing $\mathrm{TNF} \alpha$ following MSU stimulation in vitro, there was no reduction in TNF $\alpha$ in macrophage-depleted mice suggesting an alternative primary source for TNF $\alpha$ in vivo - perhaps mast cells which contain pre-formed granules of TNF $\alpha$ are also known to be important in gouty inflammation (Getting et al., 1997, Gordon and Galli, 1990). In addition, there were still small quantities of IL-1 $\beta$ and IL-6 produced in macrophage-depleted mice following MSU treatment. This was most likely derived from a small population of pro-inflammatory F4/80 monocyte/macrophages migrating into the peritoneum following clodronate treatment. Despite the presence of other cells that contribute TNF $\alpha$ in vivo, the overall reduction in cytokines and neutrophil recruitment following macrophage 
depletion confirms these cells as being key in the initiation and driving of MSUinduced inflammation.

In previous research, one group proposed that monocytes contribute to the gout inflammatory response by producing pro-inflammatory cytokines in response to MSU, while mature macrophages instead produce anti-inflammatory cytokines (Dalbeth and Haskard, 2005). Under this model it would be hypothesized that resident macrophages - a mature macrophage cell type - would produce antiinflammatory cytokines upon exposure to MSU. Since the literature at large has reported conflicting evidence to suggest that macrophages can elicit both pro- and anti-inflammatory responses to MSU crystals (Section 1.10.11.1), it is surprising that in vivo confirmation of the hypothesis of pro-inflammatory monocytes and antiinflammatory macrophages has not been sought until now. In contrast to the hypothesis of an anti-inflammatory function of macrophages, and in support of other groups reporting a pro-inflammatory response from MSU-stimulated macrophages in vitro, my findings clearly demonstrate that macrophages play a key proinflammatory role in the induction of gouty inflammation.

The conflicting results previously reported in the literature from a number of in vitro experiments highlight that mononuclear phagocytes can exhibit very different functions depending on the conditions used by researchers. As such, care needs to be taken when interpreting data derived from in vitro results especially when they are generated from "artificial" cell types. It had been previously shown that macrophages derived from in vitro differentiated $\mathrm{CD} 14^{+}$monocytes produced antiinflammatory TGF $\beta$ following exposure to MSU (Landis et al., 2002, Yagnik et al., 2004). In contrast, my results showed that resident macrophages produced proinflammatory cytokines (IL-1 $\beta$, IL-6, TNF $\alpha$ ) in response to MSU consistent with previous reports showing pro-inflammatory activities from MSU-stimulated macrophages, such as the activation of the NALP3 inflammasome.

Meanwhile, a number of other studies have routinely used other types of macrophages to study MSU-induced inflammation with varying results, further showing that macrophages can exhibit very different responses to MSU depending 
on their phenotype. For example, researchers have used macrophages elicited from an injection of thioglycollate broth into the peritoneum of mice, and these macrophage-like cells have often required exposure to priming amounts of additional stimuli such as LPS and muramyl dipeptide in order to elicit the desired IL-1 $\beta$ response on exposure to MSU (Martinon et al., 2006). The response observed by resident macrophages in my research contrasted the response of thioglycollateelicited macrophages reported by others, in that resident macrophages did not require pre-exposure to an alternative priming stimulus in order to produce active IL-1 $\beta$. My studies have also shown functional differences between resident macrophages and (immature) macrophages that have newly differentiated from inflammatory monocytes. These immature macrophages did not exhibit the same capacity as resident macrophages to produce pro-inflammatory cytokines to MSU until several days following differentiation. Although it may be possible that the differences in newly differentiated and mature resident macrophages may have completely appropriate functional consequences, these functions remain to be determined.

It is also routine to produce macrophages in vitro from mouse bone marrow progenitor cells and human blood monocytes by exposure to GM-CSF and M-CSF over several days in vitro for study in MSU-induced or other kinds of inflammation. Although these cells feature in a number of studies, it has yet to be determined how closely these cells represent true primary macrophages in MSU induced inflammation.

Collectively these data emphasize the high level of variability in macrophage responses that make it necessary to choose model systems that most accurately reflect the macrophage cell type of interest. The use of primary macrophages in this study removed several elements that would potentially have had spurious effects on macrophage responses, such as effects introduced by irrelevant inflammatory stimuli such as thioglycollate, or the presence of cytokines such as GM-CSF and M-CSF in concentrations much higher than would be found in vivo. Therefore, the use of primary macrophage cell types is a few steps closer to physiological relevancy. 
The importance of macrophage activation in the onset of gout attacks identifies macrophages as a possible target for therapy in gout-susceptible individuals. It also raises the question as to whether differences in individual susceptibilities to gout may be due to differences in the responsiveness of macrophages between individuals. There is some evidence to suggest that blood monocytes from hyperuricemic patients that do not develop gout are less responsive to MSU than monocytes from normal patients, implying that these particular hyperuricemic individuals are protected from gout due to the low responsiveness of their monocytes (Schreiner et al., 2000). However, there is as of yet no data to suggest that this is the case with macrophage cells. It would be interesting to determine whether differing responses of macrophages from asymptomatic hyperuricemic and gouty individuals to MSU would explain the enigmatic phenomenon that some hyperuricemic individuals contract gout, while others do not.

In the peritoneal model of MSU-induced inflammation, macrophages become active and adhere to MSU crystals and the surrounding tissue. However, there is no evidence to suggest that adherent macrophages detach to reappear in the peritoneal cavity. In fact, even when resident macrophages were pre-labelled with PKH26, there was no re-appearance of $\mathrm{PKH} 26^{\mathrm{hi}}, \mathrm{F} 4 / 80^{\mathrm{hi}}$ macrophages as far as a week after MSU treatment. Thus it remains to be determined whether these adherent, activated macrophages die, migrate, or assist in the resolution of inflammation while still adhering to MSU or surrounding tissue.

\subsection{Monocytes in gouty inflammation}

Until now, a study into the differentiation and functional phenotype of monocytes during the course of MSU-induced inflammation in vivo has not been undertaken. Here, I report that monocytes recruited in gout are macrophage precursors. When monocytes are recruited, they go through at least two stages of differentiation. The first is marked by a loss in the expression of Gr-1 and 7/4 within the first 24 hours. At this stage monocytes begin to take on the appearance of macrophages cells, increasing in size and forming a more circular shaped nucleus. From 3-7 days these macrophages go through another phase of differentiation marked by an upregulation of F4/80 expression to the same level that is observed on mature resident macrophages. These results contrast other reports where inflammatory monocytes 
recruited to L. monocytogenes infection, or monocytes that take up beads in the skin and travel to the lymph node, differentiate instead into DCs (Auffray et al., 2007, Randolph et al., 1999).

The differentiation of monocytes into immature macrophages and then to mature macrophages is also associated with functional changes. Recruited monocytes do not produce inflammatory cytokines such as IL- $1 \beta$, TNF $\alpha$ or IL-6 in response to MSU when first recruited in the peritoneum. From 24 hours onwards, as immature macrophages, these cells become increasingly responsive to MSU, producing IL-1 $\beta$, TNF $\alpha$ and IL-6 following exposure. In addition to this increased ability for MSUstimulated cytokine production, monocytes also develop in their capacity for phagocytosis. When monocytes are first recruited, they show very little phagocytic capacity, but this increases to a much higher capacity by 24 hours. An additional increase in phagocytic capacity occurs when these cells become F4/80 ${ }^{\text {hi }}$. These $\mathrm{F} 4 / 80^{\mathrm{hi}}$ macrophages exhibit a similar phagocytic capacity to resident macrophages, adding weight to the idea that the acquisition of an $\mathrm{F} 4 / 80^{\mathrm{hi}}$ phenotype marks a mature macrophage in the in vivo model. In combination with the surface marker information, these data outline the first extensive characterisation of monocyte differentiation in MSU-induced inflammation in vivo.

One of the novel findings from my experiments is the lack of monocyte cytokine production (IL-1 $\beta$, TNF $\alpha$, IL-6) in response to MSU within the first 16 hours of recruitment. The inability to produce these cytokines in response to MSU does not appear to be dependent on the stimulus, as both in vivo MSU- and thioglycollaterecruited cells did not produce IL-1 $\beta$, TNF $\alpha$ and IL-6 in response to MSU stimulation in vitro. Therefore, it would appear that recruited monocytes are, in general, unable to respond to MSU within the first few hours of recruitment.

It has recently been shown that the phagocytic uptake of MSU crystals and the subsequent rupture of endosomes is important for the activation of the inflammasome and the production of IL-1 $\beta$ (Hornung et al., 2008). My study shows that monocytes enter the peritoneum with low phagocytic capacity, and this lack of phagocytic capacity may prevent the uptake of crystals required for the rupture of 
endosomes that activates the inflammasome. Consistent with this, the acquisition (or reacquisition) of a high phagocytic capacity between 16-24 hours seems to correlate with the development of a pro-inflammatory response to MSU. Confirmation of a requirement for phagocytic capacity could be achieved in future experiments through the use of the phagocytosis inhibitor, cytochalasin B (MacLean-Fletcher and Pollard, 1980). If phagocytosis were required for responsiveness, then the use of this inhibitor from $24 \mathrm{~h}$ onwards would inhibit responses from newly differentiating macrophages.

It is as of yet unknown whether rupture of endosomes is required for the production of other cytokines, such as TNF $\alpha$ and IL-6. My study suggests that the ability of monocytes to produce the cytokines in response to MSU emerges for all cytokines at approximate 24 hours after MSU treatment, indicating that a similar process for MSU recognition may be required for the production of all of the cytokines measured. The use of bafilomycin to stabilise the acidification of lysosomes, would confirm whether the rupture of acidified endosomes containing MSU is indeed required for responsiveness to MSU in the production of cytokines and chemokines other than IL-1 $\beta$ (Crider et al., 1994 ). Besides activation through endosome rupturing, it remains possible that there may be cell activation or priming through receptor recognition of MSU, where absence of this unknown receptor on newly infiltrated monocytes prevents monocytes from responding to MSU.

Even though newly recruited monocytes were not responsive to MSU, they were, in fact, sensitive to LPS stimulation. MSU recruited monocytes were able to produce IL-6 and TNF $\alpha$ in response to LPS, demonstrating that monocytes were physically capable of producing cytokines. This suggests that MSU that is formed under normal physiological conditions as a "danger signal" is simply a means of inducing cellular recruitment through the production of IL-1 $\beta$. In an ordinary infectious response, a second inflammatory stimulus would also be present - bacteria with bacterial cell wall products, for example - to help stimulate additional inflammatory events. MSU crystals in gout appear to become walled off from incoming cells, hence, recruited cells may lack a secondary stimulus to stimulate the on-going 
inflammation. This lack of a secondary stimulus may contribute, at least in part, to the spontaneous resolution observed in gout in humans after 7-10 days.

\subsection{Resolution of gouty inflammation}

Increased phagocytic ability of immature macrophages coincides with the onset of neutrophil apoptosis in vivo, such that as immature macrophages become phagocytic, they ingest apoptotic neutrophils. Immature monocytes stained for MPO showed an increased positive staining as neutrophils were cleared from the peritoneum. In addition, uptake of neutrophils by immature $\mathrm{F} 4 / 80^{\mathrm{lo}}$ macrophages made them less responsive to MSU as well as to LPS. Hence, as has been previously proposed, it is possible that the uptake of neutrophils is involved in the general suppression of inflammation (Huynh et al., 2002, Rose et al., 2006). The increase in phagocytosis of apoptotic neutrophils could possibly prevent inflammatory responses from otherwise responsive immature macrophages and potentially turn off existing cytokine producing macrophages present in the peritoneum or joint. Therefore, the uptake of phagocytic neutrophils by immature macrophages may play a major role in actively driving resolution.

Although it is thought that the resolution of inflammatory processes in gout involves the generation of TGF $\beta$, in the in vivo model there was a low level of TGF $\beta$ present under normal homeostatic conditions that reduced following MSU administration, implying that any latent level of suppression being exerted by TGF $\beta$ was released following MSU challenge. However, an increase in TGF $\beta$ levels to re-establish the normal homeostatic condition was not observed during resolution in vivo, despite a decline in local cytokine levels and leukocyte numbers. Although this does not exclude TGF $\beta$ from functioning during resolution within this system, it does suggest that more in vivo work is required to determine whether TGF $\beta$ has is present and exerting any effect.

\subsection{Neutrophils in gout}

Neutrophils have been recognised for several decades as an important cell in gout. The results presented in this thesis add further insight into the activation and role of neutrophils in MSU-induced inflammation. 
Previous gout research has focussed on the activation of neutrophils following contact with MSU. My research instead has tested the hypothesis that neutrophils are also exposed to inflammatory mediators and signals prior to MSU contact that activate neutrophils completely independent of contact with MSU crystals. The effect of soluble mediators was tested on three aspects of neutrophil activation: IL-8 production, viability and superoxide production.

It has long been known that MSU causes a delay in neutrophil apoptosis (Akahoshi et al., 1997). It is also known that cytokines and chemokines affect neutrophil apoptosis; for instance, IL-8 and IL-6 delay it, while TNF $\alpha$ hastens it (Biffl et al., 1996, Kettritz et al., 1997, Kettritz et al., 1998). In my experiments, exposure of neutrophils to conditioned media containing a mixture of soluble inflammatory mediators (including IL-8, IL-6, IL-1 $\beta$ and TNF $\alpha$ ) caused a much greater delay in neutrophil apoptosis than direct contact with MSU. In fact, the improvement in viability (i.e. delay in apoptosis) did not increase any further when contact with MSU was also included as a second stimulus. Perhaps it is not surprising that an improvement in viability is observed in the presence of soluble mediators, however, this study is the first to indicate that neutrophil viability in MSU-induced inflammation may be more heavily influenced by soluble mediators than by direct MSU stimulation.

Similarly, both MSU and cytokines have been shown independently to stimulate IL8 production from neutrophils (Hachicha et al., 1995, Strieter et al., 1992). Again, my experiments showed that soluble mediators, rather than direct stimulation with MSU, had the greater effect on IL-8 production in neutrophils which further demonstrates the gathering evidence that neutrophil activation is affected by the cytokine environment, at least as much - if not more - than contact with MSU crystals. 
Soluble mediators were not able to stimulate superoxide production, showing a requirement for MSU contact; however, exposure to soluble mediators primes superoxide production in response to MSU and thus soluble mediators influence the magnitude of the superoxide response. This is similar to other neutrophil responses that have shown that IL-8, IL-1 $\beta$, TNF $\alpha$ and IL-6 can prime neutrophil superoxide production in response to other stimuli, such as fMLP (Bajaj et al., 1992, Daniels et al., 1992, Kharazmi et al., 1989, Sullivan et al., 1989). How soluble mediators influence neutrophil sensitivity to MSU crystals is yet to be determined.

These results show that activation of neutrophils in MSU-inflammation is heavily influenced by factors independent of MSU-contact and that neutrophils responses are highly dependent on the pro-inflammatory responses generated from cells producing cytokines in response to MSU.

In addition to the pro-inflammatory effect of soluble mediators, the serum environment of neutrophils alters superoxide production. Whereas neutrophils in high serum and serum-free environments show a requirement for MSU contact, a low serum environment - as may possibly be found in the joint environment triggers superoxide production indicating that MSU contact may also be unnecessary for neutrophil activation following recruitment in vivo. Although MSU crystal contact may also play a role during gouty inflammation, the response to low serum environments suggests that neutrophils may be hardwired to respond to the environmental change that occurs during recruitment in vivo, such that superoxide production is automatic upon tissue infiltration and precedes contact with the physical inflammatory stimulus. A pre-emptive strike upon infectious organisms would be the most likely benefit of such a response. However, whether the serum or plasma environment of the knee spontaneously activates infiltrating neutrophils is dependent on the presence of the currently unknown protein or compounds in similar amounts to those observed in vitro. Therefore, it cannot entirely be ruled out that the observed stimulatory phenomenon of low serum is a spurious function of in vitro conditions.

All of these data suggest that after the initiation of inflammation in gout, by macrophages and perhaps mast cells as well, that activation of neutrophils in gout in 
vivo may not even require interaction with MSU; that neutrophil activation occurs as a result of stimulation by cytokines, possibly in combination with recruitment from the high serum environment of the blood to the low serum environment of the joint.

The activation of IL-8 production, improved viability, and a high superoxide response suggest that the principle function of neutrophils during gout inflammation is to accumulate in large numbers as ROS-producing cells in the joint. IL-8 is known to be an important cytokine in the recruitment of neutrophils (Baggiolini and Clark-Lewis, 1992); therefore the production of IL-8 by neutrophils augments the recruitment of higher numbers of neutrophils into the joint space. Since neutrophils are usually short-lived cells, the delay in apoptosis under MSU-inflammatory conditions causes an extended persistence at the inflammatory site further allowing the accumulation of large amounts of neutrophils. Using the peritoneal model of gout, an acute accumulation of neutrophils was observed following MSU administration, and in addition, recruited neutrophils actively produced superoxide ex vivo without an additional stimulation and in the absence of serum in the culture conditions, indicating that recruited neutrophils were active superoxide producers upon recruitment. Therefore, it appears that superoxide production is a major inflammatory activity of neutrophils in vivo.

The idea that superoxide production is one of the main inflammatory functions of neutrophils in gout is supported by the observation that, other than IL-8, neutrophils were not a major source of other inflammatory cytokines following MSU stimulation. Unlike human mononuclear cells, purified human blood neutrophils did not produce IL-1 $\beta$, TNF $\alpha$ or IL-6 upon exposure to MSU. In support of this finding, purified MSU-recruited mouse neutrophils were also shown not to produce IL-1 $\beta$, TNF $\alpha$ or IL-6 upon re-exposure to MSU in vitro. Although it is possible that other mediators may be released from neutrophils that are not being measured in these experiments (e.g. MIP, S100 proteins), cytokine production does not appear to be a principle function of recruited neutrophils in MSU inflammation.

A limitation of the in vivo mouse model that prevented further investigation of IL-8 in gout is that mice do not produce IL-8. It could be argued that other IL-8R 
agonists in mice such as KC and MIP-1 could be used to study the role of IL- 8 in human neutrophil inflammation. However, humans also contain KC and MIP-1, so it is likely that the study of these cytokines in mice would simply model human regulation of $\mathrm{KC}$ and $\mathrm{MCP}-1$ rather than IL-8. Nevertheless, the parallel findings in purified human and mouse neutrophils of a lack of cytokine production combined with heightened superoxide production support superoxide production rather than cytokine production as the primary role for neutrophils.

\subsection{Inhibiting superoxide production as a therapy for gouty inflammation}

The principle role of neutrophils as ROS-generating cells opened up the opportunity to develop anti-inflammatory compounds aimed at inhibiting neutrophil superoxide production. This is particularly relevant in gout since MSU is a sterile inflammatory stimulus, making the generation of anti-microbial ROS physiologically irrelevant. Therefore, I investigated whether superoxide production could be inhibited by sesquiterpene dialdehyde compounds. Sesquiterpene dialdehydes were able to inhibit superoxide production from human neutrophils stimulated with both PMA and MSU crystals in vitro. These compounds were able to inhibit superoxide production in vivo when administered prior to MSU crystal administration in the mouse model of gout confirming these compounds to be potential antiinflammatories to neutrophil activation in vivo.

In addition to the inhibition of superoxide production, some sesquiterpene dialdehydes also inhibited neutrophil recruitment in vivo. Since neutrophil activation is heavily influenced by cytokines produced from MSU-responsive cells such as macrophages, this implied that the compounds were affecting other cellular targets involved in neutrophil recruitment. My results showed that sesquiterpene dialdehydes were able to inhibit MSU-stimulated cytokine production from macrophages in vitro, and were also able to inhibit NADPH oxidase activity in superoxide-producing epithelial cells: two processes known to be associated with neutrophils recruitment. Thus, these studies identify three promising sesquiterpene dialdehydes as compounds for development as anti-inflammatories targeting not only superoxide production, but also neutrophil recruitment. 
Despite being involved in the pro-inflammatory phase of gout inflammation, neutrophils are also involved in the resolution phase of gout. As mentioned above, neutrophils undergoing apoptosis are taken up by (immature) macrophages and this process inhibits the responsiveness of macrophages to MSU and also to LPS allowing resolution of inflammation. It has yet to be directly determined whether this process is also responsible for the reduction of cytokine production observed in vivo during the resolution of the cytokine response where reduction in proinflammatory cytokine production occurs before maximal cell infiltration.

\subsection{Dissecting the roles of inflammatory cells in gout}

The major body of this thesis was focussed on the role of monocytes, macrophages and neutrophils to the MSU-inflammatory response, and as such, the contributions of each of these cell types has been outlined in this discussion thus far. The outcomes of this research also revealed potential contributions of epithelial cells to MSUinduced inflammation.

MSU crystals were able to stimulate epithelial cells to produce MCP-1, both as whole excised epithelial tissue and as purified cells cultures. When compared against stimulated resident leukocytes, epithelial tissue was the main source of MSU-stimulated MCP-1 over the acute cytokine-producing period. As MCP-1 is important in the recruitment of monocytes, this implies that epithelial cells may be responsible for monocyte recruitment. In line with this observation, monocyte recruitment was unaffected by macrophage depletion, indicating that cells other than macrophages were responsible for monocyte recruitment.

It is also possible that superoxide production by epithelial cells is somehow involved in MSU-induced cellular recruitment. I have shown that sesquiterpene compounds with anti-inflammatory activity against NADPH oxidase were able to inhibit the production of superoxide from stimulated HUVECs, and this activity may have contributed to the reduced neutrophil recruitment. Superoxide production by MSUstimulated HUVECs has been previously reported (Falasca et al., 1993) and HUVECs exposed to MSU promoted neutrophil adhesion to these endothelial cells (Reinhardt et al., 1996). Hence there is a growing understanding that MSUstimulated epithelial cells, including endothelium, contribute to the recruitment of 
monocytes and/or neutrophils during the gout inflammatory response. Further research into the contribution of epithelial cells in cellular recruitment would help to identify which arm of the inflammatory response is affected or initiated by these cells.

\subsection{Future directions}

The findings of this study filter into an important question that remains to be answered in gouty arthritis; that is: why do $75 \%$ of individuals with hyperuricemia not develop gout (Campion et al., 1987)? The answer to this question is particularly important for groups such as Maori, who suffer a particularly high incidence of gout, and therefore may be a part of the $25 \%$ of hyperuricemics that are prone to gout. If it can be determined what aspects influence risk in hyperuricemic conditions, then diagnostic tests may be developed to identify at risk individuals, and new treatments developed for both prophylaxis and disease mitigation. But which biological elements are likely candidates for exploring the above question?

The results of this study identify a number of elements that may be explored in future studies. For instance, resident macrophages were shown in Chapter 5 to be important in the induction of gouty arthritis: could it be that macrophages of people who develop gout can detect very small quantities of MSU crystals? Support for a role of macrophages in exerting control over responses to MSU is also found in Chapter 3, where soluble mediators in the surrounding milieu influenced neutrophil function in response to MSU. Therefore, it may also be possible that macrophages from individuals that develop gout may produce higher amounts of proinflammatory cytokines in response to MSU, leading to exacerbated inflammatory responses from downstream inflammatory players such as neutrophils.

The importance of soluble mediators on neutrophil function also begs the question of whether sufferers of gout have a higher than normal systemic level of particular proinflammatory cytokines that may cause neutrophils to exist in a primed state even during normal homeostasis. An extensive clinical study of this has not been reported, and as such, this kind of study may help to explain why gouty attacks appear to become more acute and intense as gouty attacks increase. 
The effect of hyperuricemia on inflammatory responses was not a subject of this thesis, yet may itself be playing a role in gout over and above providing a pool of uric acid from which MSU crystals may be formed. It is possible that the responses of macrophages - and indeed, of other cells including neutrophils - may be affected by the presence of high soluble uric acid. Soluble uric acid may be having an effect on leukocyte responses in vivo that has yet to be demonstrated in the literature. Since mice have functional uricase, and uricase knockout mice develop severe pathology and high lethality, the use of a murine model for responses in hyperuricemic conditions has not been extensively studied. Recently, the use of the uricase inhibitor, oxonic acid, to induce hyperuricemia in rats has been reported, opening the way to study the effect of hyperuricemia on MSU-induced inflammation (Mazzali et al., 2001). Although this model is likely to yield interesting results including the secondary effects of hyperuricemia on other metabolic disorders hyperuricemia is a common factor for both hyperuricemia groups that either contract and do not contract gout, and therefore it is not likely that hyperuricemia is the sole cause of the exacerbated responses observed in gouty individuals.

The results of Chapter 6 show that monocytes differentiate into macrophages, which then ingest apoptotic neutrophils, and as a result, the uptake of neutrophils prevents pro-inflammatory responses. Many studies have shown that the uptake of apoptotic neutrophils causes the release of TGF $\beta$ that contributes to the observed resolution (Fadok et al., 1998, Huynh et al., 2002). I was unable to detect high enough levels of TGF $\beta$ using the procedures available to me; however, future work would involve alternative methods of measuring TGF $\beta$ including quantitative PCR to measure the levels of TGF $\beta$ message. This might help determine whether the production of TGF $\beta$ by mononuclear phagocytes truly plays a role in resolution.

One of the principal reasons for interest in the processes surrounding resolution in gout is that if it was known what processes control resolution, then these processes could be used to expedite and even prevent gouty attacks. It is entirely possible that pro-resolution processes that do not involve TGF $\beta$ may also be at play. It was recently shown that apoptotic neutrophils upregulate chemokine receptors while undergoing apoptosis, sequestering chemokines via these receptors and then being 
taken up as a whole by macrophages (Ariel et al., 2006). This process was hypothesised to be important in the clearance of chemokines during inflammatory resolution. Whether this process also occurs during resolution in gouty arthritis is a subject for future investigation.

\subsection{Conclusion}

This research, focussed on the innate immune cells involved in the gout response, enables a new overview of acute gout inflammation to be drawn that outlines the initiation, progression, and resolution of a gout attack.

The formation of MSU crystals activates macrophages to produce pro-inflammatory cytokines such as IL-1 $\beta$, TNF $\alpha$ and IL-6. Epithelial cells are also activated and produce MCP-1. The production of these cytokines produced by MSU-stimulated cells initiates the recruitment of cells into the joint (Fig. 7.1A).

Neutrophils are recruited in response to the production of macrophage-derived cytokines. Both in the blood and in the joint, neutrophils are exposed to elevated cytokine levels and this exposure leads them to produce IL-8, delay homeostatic apoptosis and primes them for superoxide production, all potentially before contact with MSU. As neutrophils infiltrate into the joint, the movement from the high serum environment of the bloodstream to the low serum environment of the joint may further augment the production of superoxide that occurs following neutrophil interaction with MSU, adding to the inflammatory environment in the joint (Fig. 7.1B).

Monocytes are also recruited to the joint, through a process that is not dependent on macrophage activation. Monocytes infiltrate into the joint but do not produce proinflammatory cytokines. By the time monocytes have differentiated into immature macrophages capable of responding to MSU crystals, MSU in the joint has already been walled off such that monocytes do not interact with MSU. Instead, as 

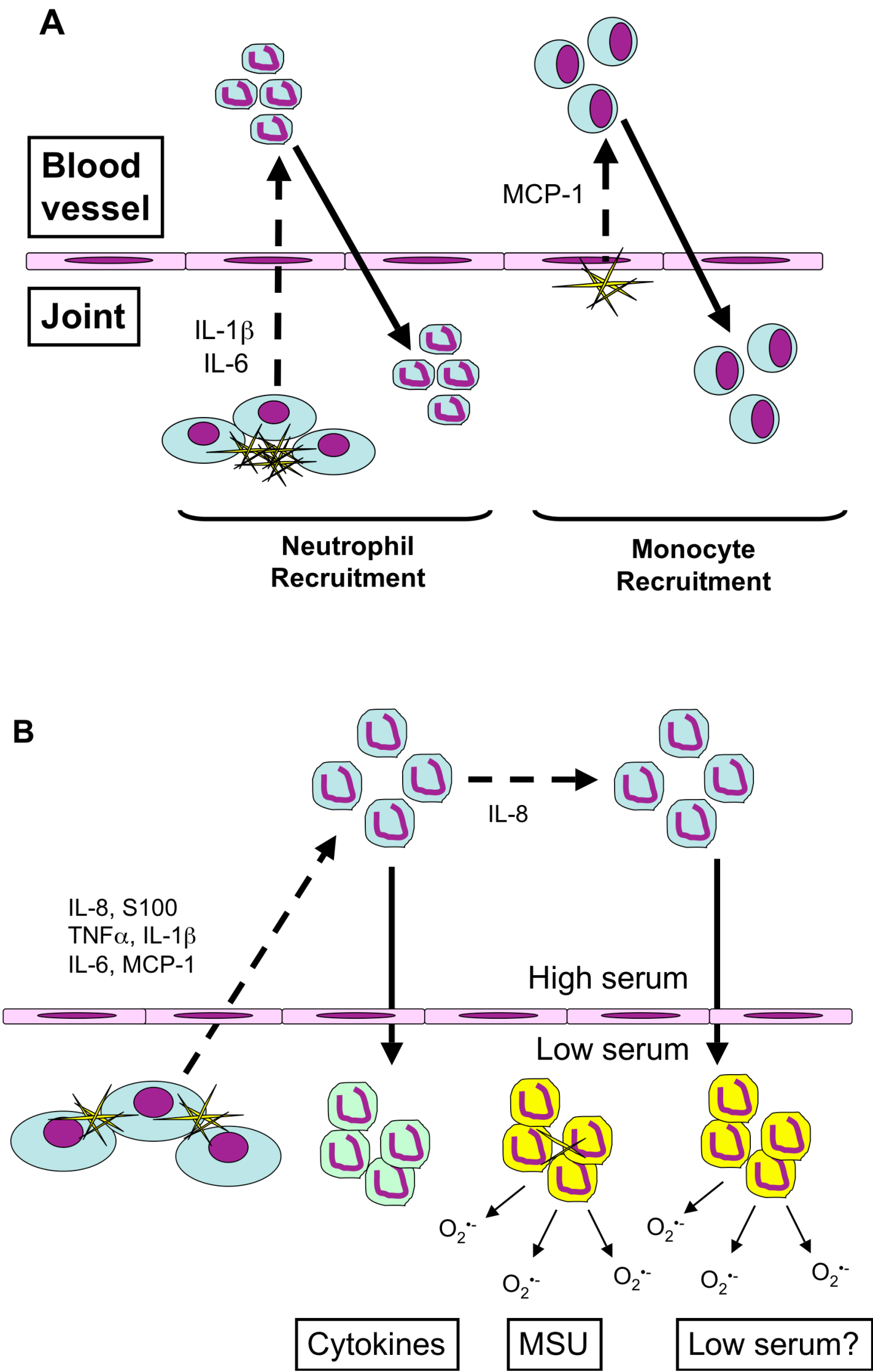


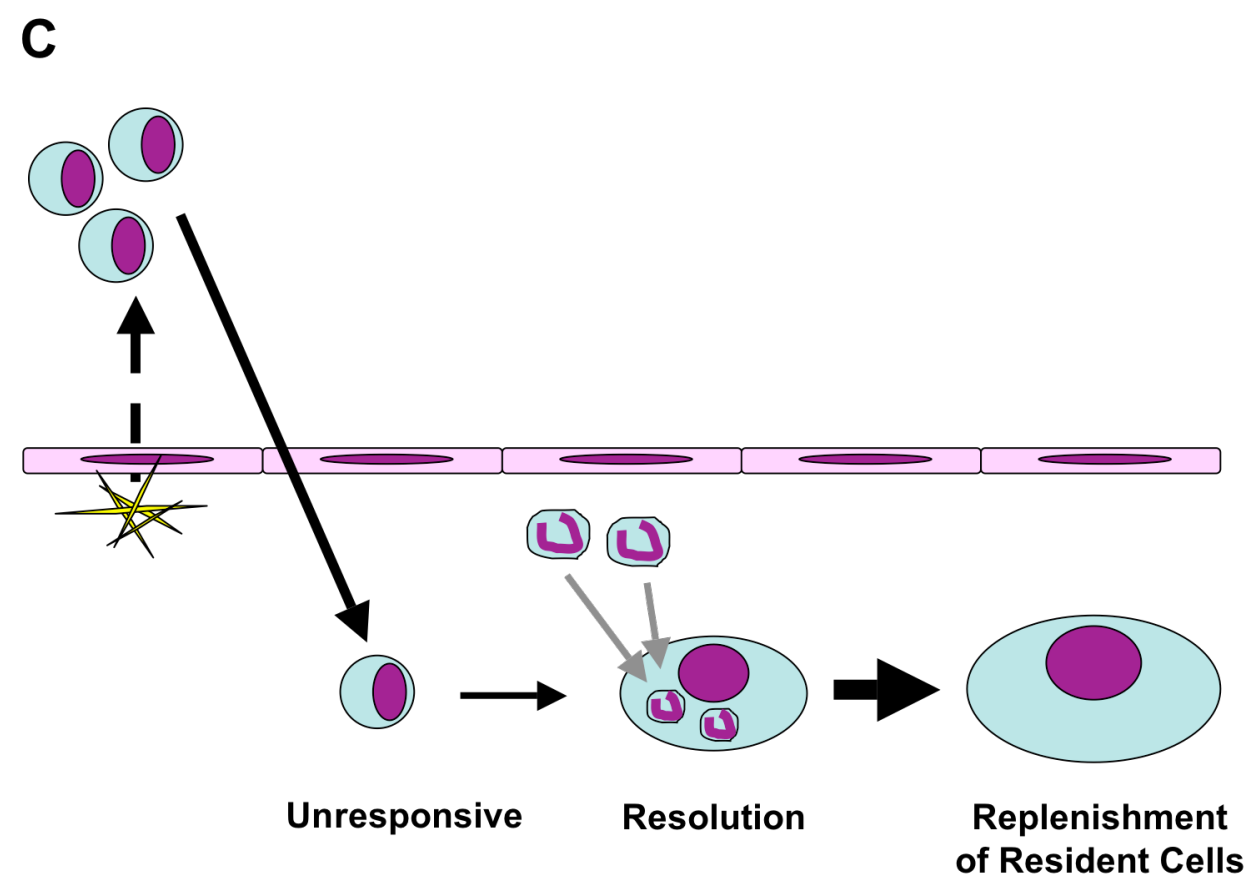

Figure 7.1 Model of acute gouty inflammation.

A. MSU crystals stimulate the induction of acute gout inflammation, resulting in the recruitment of neutrophils and monocytes. Resident macrophages produce proinflammatory cytokines including IL-1 $\beta$ and IL-6 that result in the neutrophil recruitment. Monocyte recruitment occurs via a separate mechanism that potentially involves the production of MCP-1 by endothelial cells. B. During MSU-induced inflammation, neutrophils are exposed to pro-inflammatory cytokines, which causes them to become activated (green), producing IL-8 and delaying apoptosis which augments the accumulation of neutrophils in large numbers prior to contact with MSU. Recruited neutrophils are exposed to at least three inflammatory stimuli in the joint. The first, is cytokine exposure that leads to activation and priming (green). The second is physical contact of cytokine-primed neutrophils with MSU, resulting an intense production of superoxide (yellow). The third stimulus is the movement into a low serum environment resulting in superoxide production independent of MSU contact (yellow). C. Monocytes recruited to MSU-induced inflammation do not produce large amounts of cytokines upon recruitment, nor are they "responsive" to MSU during the inflammatory phase. Instead, they differentiate into immature macrophages and participate in the resolution of inflammation by phagocytosing apoptotic neutrophils. After several days, immature macrophages differentiate into resident macrophages, replenishing the resident population once inflammation has subsided. 
immature macrophages they phagocytose the neutrophils that have been recruited and have become apoptotic. This uptake of neutrophils further prevents their ability to respond to MSU. The uptake of neutrophils by cytokine producing cells may also turn off existing cytokine production, facilitating resolution. After approximately a week, immature macrophages differentiate into resident-like macrophages replenishing the local resident population (Fig. 7.1C).

In summary, this research provides significant clarification on the roles of monocytes, macrophages and neutrophils in gout and dissects their various contributions and interactions in gout inflammation over the course of an MSUinduced response. 


\section{Chapter 8}

\section{References}

ABRAMSON, S., HOFFSTEIN, S. T. \& WEISSMANN, G. (1982) Superoxide anion generation by human neutrophils exposed to monosodium urate. Arthritis Rheum, 25, 174-80.

ADAIR-KIRK, T. L., ATKINSON, J. J., BROEKELMANN, T. J., DOI, M., TRYGGVASON, K., MINER, J. H., MECHAM, R. P. \& SENIOR, R. M. (2003) A site on laminin alpha 5, AQARSAASKVKVSMKF, induces inflammatory cell production of matrix metalloproteinase- 9 and chemotaxis. J Immunol, 171, 398-406.

AGO, T., KITAZONO, T., OOBOSHI, H., IYAMA, T., HAN, Y. H., TAKADA, J., WAKISAKA, M., IBAYASHI, S., UTSUMI, H. \& IIDA, M. (2004) Nox4 as the major catalytic component of an endothelial $\mathrm{NAD}(\mathrm{P}) \mathrm{H}$ oxidase. Circulation, 109, 227-33.

AHERN, M. J., REID, C., GORDON, T. P., MCCREDIE, M., BROOKS, P. M. \& JONES, M. (1987) Does colchicine work? The results of the first controlled study in acute gout. Aust N Z J Med, 17, 301-4.

AKAHOSHI, T., NAGAOKA, T., NAMAI, R., SEKIYAMA, N. \& KONDO, H. (1997) Prevention of neutrophil apoptosis by monosodium urate crystals. Rheumatol Int, 16, 231-5.

AKGUL, C., MOULDING, D. A. \& EDWARDS, S. W. (2001) Molecular control of neutrophil apoptosis. FEBS Lett, 487, 318-22.

AKIRA, S., TAKEDA, K. \& KAISHO, T. (2001) Toll-like receptors: critical proteins linking innate and acquired immunity. Nat Immunol, 2, 675-80.

AMES, B. N., CATHCART, R., SCHWIERS, E. \& HOCHSTEIN, P. (1981) Uric acid provides an antioxidant defense in humans against oxidant- and radicalcaused aging and cancer: a hypothesis. Proc Natl Acad Sci U S A, 78, 685862.

ANNANMAKI, T., MUURONEN, A. \& MURROS, K. (2007) Low plasma uric acid level in Parkinson's disease. Mov Disord, 22, 1133-7.

ARIEL, A., FREDMAN, G., SUN, Y. P., KANTARCI, A., VAN DYKE, T. E., LUSTER, A. D. \& SERHAN, C. N. (2006) Apoptotic neutrophils and T cells sequester chemokines during immune response resolution through modulation of CCR5 expression. Nat Immunol, 7, 1209-16.

ATHENS, J. W., HAAB, O. P., RAAB, S. O., MAUER, A. M., ASHENBRUCKER, H., CARTWRIGHT, G. E. \& WINTROBE, M. M. (1961) Leukokinetic studies. IV. The total blood, circulating and marginal granulocyte pools and the granulocyte turnover rate in normal subjects. J Clin Invest, 40, 989-95.

AUFFRAY, C., FOGG, D., GARFA, M., ELAIN, G., JOIN-LAMBERT, O., KAYAL, S., SARNACKI, S., CUMANO, A., LAUVAU, G. \& GEISSMANN, F. (2007) Monitoring of blood vessels and tissues by a population of monocytes with patrolling behavior. Science, 317, 666-70. 
AUSTYN, J. M. \& GORDON, S. (1981) F4/80, a monoclonal antibody directed specifically against the mouse macrophage. Eur J Immunol, 11, 805-15.

BABIOR, B. M. (2000) Phagocytes and oxidative stress. Am J Med, 109, 33-44.

BAGGIOLINI, M. \& CLARK-LEWIS, I. (1992) Interleukin-8, a chemotactic and inflammatory cytokine. FEBS Lett, 307, 97-101.

BAJAJ, M. S., KEW, R. R., WEBSTER, R. O. \& HYERS, T. M. (1992) Priming of human neutrophil functions by tumor necrosis factor: enhancement of superoxide anion generation, degranulation, and chemotaxis to chemoattractants C5a and F-Met-Leu-Phe. Inflammation, 16, 241-50.

BANCHEREAU, J. \& STEINMAN, R. M. (1998) Dendritic cells and the control of immunity. Nature, 392, 245-52.

BARABE, F., GILBERT, C., LIAO, N., BOURGOIN, S. G. \& NACCACHE, P. H. (1998) Crystal-induced neutrophil activation VI. Involvment of FcgammaRIIIB (CD16) and CD11b in response to inflammatory microcrystals. Faseb J, 12, 209-20.

BECKER, B. F. (1993) Towards the physiological function of uric acid. Free Radic Biol Med, 14, 615-31.

BECKER, M. A., SCHUMACHER, H. R., JR., WORTMANN, R. L., MACDONALD, P. A., EUSTACE, D., PALO, W. A., STREIT, J. \& JOSEPH-RIDGE, N. (2005) Febuxostat compared with allopurinol in patients with hyperuricemia and gout. $N$ Engl J Med, 353, 2450-61.

BELlingAN, G. J., CALDWELL, H., HOWIE, S. E., DRANSFIELD, I. \& HASLETT, C. (1996) In vivo fate of the inflammatory macrophage during the resolution of inflammation: inflammatory macrophages do not die locally, but emigrate to the draining lymph nodes. J Immunol, 157, 2577-85.

BIFFL, W. L., MOORE, E. E., MOORE, F. A., BARNETT, C. C., JR., CARL, V. S. \& PETERSON, V. N. (1996) Interleukin-6 delays neutrophil apoptosis. Arch Surg, 131, 24-9; discussion 29-30.

BLUMBERG, P. M. (1988) Protein kinase C as the receptor for the phorbol ester tumor promoters: sixth Rhoads memorial award lecture. Cancer Res, 48, 1-8.

BOMALASKI, J. S. \& CLARK, M. A. (2004) Serum uric acid-lowering therapies: where are we heading in management of hyperuricemia and the potential role of uricase. Curr Rheumatol Rep, 6, 240-7.

BONNEL, R. A., VILlAlBA, M. L., KARWOSKI, C. B. \& BEITZ, J. (2002) Deaths associated with inappropriate intravenous colchicine administration. $J$ Emerg Med, 22, 385-7.

BOOGAERTS, M. A., HAMMERSCHMIDT, D. E., ROELANT, C., VERWILGHEN, R. L. \& JACOB, H. S. (1983) Mechanisms of vascular damage in gout and oxalosis: crystal induced, granulocyte mediated, endothelial injury. Thromb Haemost, 50, 576-80.

BOT, J., WHITAKER, D., VIVIAN, J., LAKE, R., YAO, V. \& MCCAULEY, R. (2003) Culturing mouse peritoneal mesothelial cells. Pathol Res Pract, 199, 341-4.

BURT, H. M. \& JACKSON, J. K. (1997) The priming action of tumour necrosis factor-alpha (TNF-alpha) and granulocyte-macrophage colony-stimulating factor (GM-CSF) on neutrophils activated by inflammatory microcrystals. Clin Exp Immunol, 108, $432-7$.

CAILHIER, J. F., PARTOLINA, M., VUTHOORI, S., WU, S., KO, K., WATSON, S., SAVILL, J., HUGHES, J. \& LANG, R. A. (2005) Conditional 
macrophage ablation demonstrates that resident macrophages initiate acute peritoneal inflammation. J Immunol, 174, 2336-42.

CAMPBELL, J. J., HEDRICK, J., ZLOTNIK, A., SIANI, M. A., THOMPSON, D. A. \& BUTCHER, E. C. (1998) Chemokines and the arrest of lymphocytes rolling under flow conditions. Science, 279, 381-4.

CAMPBELL, J. J., QIN, S., BACON, K. B., MACKAY, C. R. \& BUTCHER, E. C. (1996) Biology of chemokine and classical chemoattractant receptors: differential requirements for adhesion-triggering versus chemotactic responses in lymphoid cells. $J$ Cell Biol, 134, 255-66.

CAMPION, E. W., GLYNN, R. J. \& DELABRY, L. O. (1987) Asymptomatic hyperuricemia. Risks and consequences in the Normative Aging Study. Am J Med, 82, 421-6.

CASSATELLA, M. A. (1995) The production of cytokines by polymorphonuclear neutrophils. Immunol Today, 16, 21-6.

CHAPMAN, P. T., YARWOOD, H., HARRISON, A. A., STOCKER, C. J., JAMAR, F., GUNDEL, R. H., PETERS, A. M. \& HASKARD, D. O. (1997) Endothelial activation in monosodium urate monohydrate crystal-induced inflammation: in vitro and in vivo studies on the roles of tumor necrosis factor alpha and interleukin-1. Arthritis Rheum, 40, 955-65.

CHEN, C. J., SHI, Y., HEARN, A., FITZGERALD, K., GOLENBOCK, D., REED, G., AKIRA, S. \& ROCK, K. L. (2006) MyD88-dependent IL-1 receptor signaling is essential for gouty inflammation stimulated by monosodium urate crystals. $J$ Clin Invest, 116, 2262-71.

CHIA, E. W., GRAINGER, R. \& HARPER, J. L. (2008) Colchicine suppresses neutrophil superoxide production in a murine model of gouty arthritis: a rationale for use of low-dose colchicine. Br J Pharmacol, 153, 1288-95.

CHOI, H. K., ATKINSON, K., KARLSON, E. W. \& CURHAN, G. (2005) Obesity, weight change, hypertension, diuretic use, and risk of gout in men: the health professionals follow-up study. Arch Intern Med, 165, 742-8.

CHOI, H. K., ATKINSON, K., KARLSON, E. W., WILLETT, W. \& CURHAN, G. (2004a) Alcohol intake and risk of incident gout in men: a prospective study. Lancet, 363, 1277-81.

CHOI, H. K., ATKINSON, K., KARLSON, E. W., WILLETT, W. \& CURHAN, G. (2004b) Purine-rich foods, dairy and protein intake, and the risk of gout in men. N Engl J Med, 350, 1093-103.

CHOI, H. K. \& CURHAN, G. (2004) Beer, liquor, and wine consumption and serum uric acid level: the Third National Health and Nutrition Examination Survey. Arthritis Rheum, 51, 1023-9.

CHOI, H. K. \& CURHAN, G. (2007) Coffee, tea, and caffeine consumption and serum uric acid level: the third national health and nutrition examination survey. Arthritis Rheum, 57, 816-21.

CHOI, H. K. \& CURHAN, G. (2008) Soft drinks, fructose consumption, and the risk of gout in men: prospective cohort study. BMJ, 336, 309-12.

CHOI, H. K., WILLETT, W. \& CURHAN, G. (2007) Coffee consumption and risk of incident gout in men: a prospective study. Arthritis Rheum, 56, 2049-55.

CHOI, J. W., FORD, E. S., GAO, X. \& CHOI, H. K. (2008) Sugar-sweetened soft drinks, diet soft drinks, and serum uric acid level: the Third National Health and Nutrition Examination Survey. Arthritis Rheum, 59, 109-16. 
COE, F. L., MORAN, E. \& KAVALICH, A. G. (1976) The contribution of dietary purine over-consumption to hyperpuricosuria in calcium oxalate stone formers. J Chronic Dis, 29, 793-800.

CONAGHAN, P. G. \& DAY, R. O. (1994) Risks and benefits of drugs used in the management and prevention of gout. Drug Saf, 11, 252-8.

COnstantin, G., MAJEed, M., GIAGUlLI, C., PICCIO, L., KIM, J. Y., BUTCHER, E. C. \& LAUDANNA, C. (2000) Chemokines trigger immediate beta2 integrin affinity and mobility changes: differential regulation and roles in lymphocyte arrest under flow. Immunity, 13, 759-69.

CRIDER, B. P., XIE, X. S. \& STONE, D. K. (1994) Bafilomycin inhibits proton flow through the $\mathrm{H}+$ channel of vacuolar proton pumps. J Biol Chem, 269, 17379-81.

CROSS, A. R. \& JONES, O. T. (1986) The effect of the inhibitor diphenylene iodonium on the superoxide-generating system of neutrophils. Specific labelling of a component polypeptide of the oxidase. Biochem J, 237, 111-6.

DA CUNHA, F. M., FRODE, T. S., MENDES, G. L., MALHEIROS, A., CECHINEL FILHO, V., YUNES, R. A. \& CALIXTO, J. B. (2001) Additional evidence for the anti-inflammatory and anti-allergic properties of the sesquiterpene polygodial. Life Sci, 70, 159-69.

DALBETH, N. \& HASKARD, D. O. (2005) Mechanisms of inflammation in gout. Rheumatology (Oxford), 44, 1090-6.

DAMATTA, R. A., ARAUJO-JORGE, T. \& DE SOUZA, W. (1995) Subpopulations of mouse resident peritoneal macrophages fractionated on Percoll gradients show differences in cell size, lectin binding and antigen expression suggestive of different stages of maturation. Tissue Cell, 27, 50513.

DANCEY, J. T., DEUBELBEISS, K. A., HARKER, L. A. \& FINCH, C. A. (1976) Neutrophil kinetics in man. J Clin Invest, 58, 705-15.

DANGERFIELD, J., LARBI, K. Y., HUANG, M. T., DEWAR, A. \& NOURSHARGH, S. (2002) PECAM-1 (CD31) homophilic interaction upregulates alpha6betal on transmigrated neutrophils in vivo and plays a functional role in the ability of alpha6 integrins to mediate leukocyte migration through the perivascular basement membrane. J Exp Med, 196, 1201-11.

DANIELS, R. H., FINNEN, M. J., HILL, M. E. \& LACKIE, J. M. (1992) Recombinant human monocyte IL-8 primes NADPH-oxidase and phospholipase A2 activation in human neutrophils. Immunology, 75, 157-63.

DE LEONARDIS, F., GOVONI, M., COLINA, M., BRUSCHI, M. \& TROTTA, F. (2007) Elderly-onset gout: a review. Rheumatol Int, 28, 1-6.

DESSEIN, P. H., SHIPTON, E. A., STANWIX, A. E., JOFFE, B. I. \& RAMOKGADI, J. (2000) Beneficial effects of weight loss associated with moderate calorie/carbohydrate restriction, and increased proportional intake of protein and unsaturated fat on serum urate and lipoprotein levels in gout: a pilot study. Ann Rheum Dis, 59, 539-43.

DI GIOVINE, F. S., MALAWISTA, S. E., NUKI, G. \& DUFF, G. W. (1987) Interleukin 1 (IL 1) as a mediator of crystal arthritis. Stimulation of T cell and synovial fibroblast mitogenesis by urate crystal-induced IL 1. J Immunol, 138, 3213-8.

DI GIOVINE, F. S., MALAWISTA, S. E., THORNTON, E. \& DUFF, G. W. (1991) Urate crystals stimulate production of tumor necrosis factor alpha from 
human blood monocytes and synovial cells. Cytokine mRNA and protein kinetics, and cellular distribution. J Clin Invest, 87, 1375-81.

DINCER, H. E., DINCER, A. P. \& LEVINSON, D. J. (2002) Asymptomatic hyperuricemia: to treat or not to treat. Cleve Clin J Med, 69, 594, 597, 600-2 passim.

ENOMOTO, A., KIMURA, H., CHAIROUNGDUA, A., SHIGETA, Y., JUTABHA, P., CHA, S. H., HOSOYAMADA, M., TAKEDA, M., SEKINE, T., IGARASHI, T., MATSUO, H., KIKUCHI, Y., ODA, T., ICHIDA, K., HOSOYA, T., SHIMOKATA, K., NIWA, T., KANAI, Y. \& ENDOU, H. (2002) Molecular identification of a renal urate anion exchanger that regulates blood urate levels. Nature, 417, 447-52.

FADOK, V. A., BRATTON, D. L., KONOWAL, A., FREED, P. W., WESTCOTT, J. Y. \& HENSON, P. M. (1998) Macrophages that have ingested apoptotic cells in vitro inhibit proinflammatory cytokine production through autocrine/paracrine mechanisms involving TGF-beta, PGE2, and PAF. J Clin Invest, 101, 890-8.

FAIRES, J. S. \& MCCARTY, D. J. (1962) Acute arthritis in man and dog after intrasynovial injection of sodium urate crystals. The Lancet, 2, 682.

FALASCA, G. F., RAMACHANDRULA, A., KELLEY, K. A., O'CONNOR, C. R. \& REGINATO, A. J. (1993) Superoxide anion production and phagocytosis of crystals by cultured endothelial cells. Arthritis Rheum, 36, 105-16.

FALLER, J. \& FOX, I. H. (1982) Ethanol-induced hyperuricemia: evidence for increased urate production by activation of adenine nucleotide turnover. $N$ Engl J Med, 307, 1598-602.

FEIG, D. I. \& JOHNSON, R. J. (2007) The role of uric acid in pediatric hypertension. J Ren Nutr, 17, 79-83.

FERRAZ, M. B. \& O'BRIEN, B. (1995) A cost effectiveness analysis of urate lowering drugs in nontophaceous recurrent gouty arthritis. J Rheumatol, 22, 908-14.

FLEETWOOD, A. J., LAWRENCE, T., HAMILTON, J. A. \& COOK, A. D. (2007) Granulocyte-macrophage colony-stimulating factor (CSF) and macrophage CSF-dependent macrophage phenotypes display differences in cytokine profiles and transcription factor activities: implications for CSF blockade in inflammation. J Immunol, 178, 5245-52.

FOX, I. H. \& KELLEY, W. N. (1972) Studies on the mechanism of fructose-induced hyperuricemia in man. Metabolism, 21, 713-21.

FOX, I. H., PALELLA, T. D. \& KELLEY, W. N. (1987) Hyperuricemia: a marker for cell energy crisis. $N$ Engl $J$ Med, 317, 111-2.

GAO, X., CURHAN, G., FORMAN, J. P., ASCHERIO, A. \& CHOI, H. K. (2008) Vitamin C Intake and Serum Uric Acid Concentration in Men. J Rheumatol.

GEISSMANN, F., JUNG, S. \& LITTMAN, D. R. (2003) Blood monocytes consist of two principal subsets with distinct migratory properties. Immunity, 19, 7182.

GETTING, S. J., FLOWER, R. J., PARENTE, L., DE MEDICIS, R., LUSSIER, A., WOLIZTKY, B. A., MARTINS, M. A. \& PERRETTI, M. (1997) Molecular determinants of monosodium urate crystal-induced murine peritonitis: a role for endogenous mast cells and a distinct requirement for endothelial-derived selectins. J Pharmacol Exp Ther, 283, 123-30. 
GIBSON, T., WATERWORTH, R., HATFIELD, P., ROBINSON, G. \& BREMNER, K. (1984) Hyperuricaemia, gout and kidney function in New Zealand Maori men. Br J Rheumatol, 23, 276-82.

GILBERT, C., POUBELlE, P. E., BORGEAT, P., POULIOT, M. \& NACCACHE, P. H. (2003) Crystal-induced neutrophil activation: VIII. Immediate production of prostaglandin E2 mediated by constitutive cyclooxygenase 2 in human neutrophils stimulated by urate crystals. Arthritis Rheum, 48, 113748.

GINHOUX, F., TACKE, F., ANGELI, V., BOGUNOVIC, M., LOUBEAU, M., DAI, X. M., STANLEY, E. R., RANDOLPH, G. J. \& MERAD, M. (2006) Langerhans cells arise from monocytes in vivo. Nat Immunol, 7, 265-73.

GORDON, J. R. \& GALLI, S. J. (1990) Mast cells as a source of both preformed and immunologically inducible TNF-alpha/cachectin. Nature, 346, 274-6.

GORDON, S. (2003) Alternative activation of macrophages. Nat Rev Immunol, 3, 23-35.

GORDON, S. \& TAYLOR, P. R. (2005) Monocyte and macrophage heterogeneity. Nat Rev Immunol, 5, 953-64.

GRAGE-GRIEBENOW, E., FLAD, H. D. \& ERNST, M. (2001) Heterogeneity of human peripheral blood monocyte subsets. J Leukoc Biol, 69, 11-20.

GREENWOOD, J., AMOS, C. L., WALTERS, C. E., COURAUD, P. O., LYCK, R., ENGELHARDT, B. \& ADAMSON, P. (2003) Intracellular domain of brain endothelial intercellular adhesion molecule-1 is essential for $\mathrm{T}$ lymphocytemediated signaling and migration. J Immunol, 171, 2099-108.

GRIEBSCH, A. \& ZOLLNER, N. (1974) Effect of ribomononucleotides given orally on uric acid production in man. Adv Exp Med Biol, 41, 443-9.

GUERNE, P. A., TERKELTAUB, R., ZURAW, B. \& LOTZ, M. (1989) Inflammatory microcrystals stimulate interleukin-6 production and secretion by human monocytes and synoviocytes. Arthritis Rheum, 32, 1443-52.

HACHICHA, M., NACCACHE, P. H. \& MCCOLL, S. R. (1995) Inflammatory microcrystals differentially regulate the secretion of macrophage inflammatory protein 1 and interleukin 8 by human neutrophils: a possible mechanism of neutrophil recruitment to sites of inflammation in synovitis. $J$ Exp Med, 182, 2019-25.

HALL, A. P., BARRY, P. E., DAWBER, T. R. \& MCNAMARA, P. M. (1967) Epidemiology of gout and hyperuricemia. A long-term population study. Am JMed, 42, 27-37.

HAMANN, J., KONING, N., POUWELS, W., ULFMAN, L. H., VAN EIJK, M., STACEY, M., LIN, H. H., GORDON, S. \& KWAKKENBOS, M. J. (2007) EMR1, the human homolog of F4/80, is an eosinophil-specific receptor. Eur J Immunol, 37, 2797-802.

HAMPTON, M. B., KETTLE, A. J. \& WINTERBOURN, C. C. (1998) Inside the neutrophil phagosome: oxidants, myeloperoxidase, and bacterial killing. Blood, 92, 3007-17.

HARADA, A., SEKIDO, N., AKAHOSHI, T., WADA, T., MUKAIDA, N. \& MATSUSHIMA, K. (1994) Essential involvement of interleukin-8 (IL-8) in acute inflammation. J Leukoc Biol, 56, 559-64.

HARRIS, C. M., LLOYD, D. C. \& LEWIS, J. (1995) The prevalence and prophylaxis of gout in England. J Clin Epidemiol, 48, 1153-8. 
HASKILL, S. \& BECKER, S. (1985) Disappearance and reappearance of resident macrophages: importance in C. parvum-induced tumoricidal activity. Cell Immunol, 90, 179-89.

HEDIGER, M. A., JOHNSON, R. J., MIYAZAKI, H. \& ENDOU, H. (2005) Molecular physiology of urate transport. Physiology (Bethesda), 20, 125-33.

HENDERSON, R. B., HOBBS, J. A., MATHIES, M. \& HOGG, N. (2003) Rapid recruitment of inflammatory monocytes is independent of neutrophil migration. Blood, 102, 328-35.

HERRICK, S. E. \& MUTSAERS, S. E. (2004) Mesothelial progenitor cells and their potential in tissue engineering. Int J Biochem Cell Biol, 36, 621-42.

HIRSCH, J. G. \& COHN, Z. A. (1960) Degranulation of polymorphonuclear leucocytes following phagocytosis of microorganisms. J Exp Med, 112, 1005-14.

HOOPER, D. C., SPITSIN, S., KEAN, R. B., CHAMPION, J. M., DICKSON, G. M., CHAUDHRY, I. \& KOPROWSKI, H. (1998) Uric acid, a natural scavenger of peroxynitrite, in experimental allergic encephalomyelitis and multiple sclerosis. Proc Natl Acad Sci U S A, 95, 675-80.

HORNUNG, V., BAUERNFEIND, F., HALLE, A., SAMSTAD, E. O., KONO, H., ROCK, K. L., FITZGERALD, K. A. \& LATZ, E. (2008) Silica crystals and aluminum salts activate the NALP3 inflammasome through phagosomal destabilization. Nat Immunol, 9, 847-56.

HUANG, A. J., MANNING, J. E., BANDAK, T. M., RATAU, M. C., HANSER, K. R. \& SILVERSTEIN, S. C. (1993) Endothelial cell cytosolic free calcium regulates neutrophil migration across monolayers of endothelial cells. J Cell Biol, 120, 1371-80.

HUANG, H. Y., APPEL, L. J., CHOI, M. J., GELBER, A. C., CHARLESTON, J., NORKUS, E. P. \& MILLER, E. R., 3RD (2005) The effects of vitamin C supplementation on serum concentrations of uric acid: results of a randomized controlled trial. Arthritis Rheum, 52, 1843-7.

HUME, D. A., ROBINSON, A. P., MACPHERSON, G. G. \& GORDON, S. (1983) The mononuclear phagocyte system of the mouse defined by immunohistochemical localization of antigen F4/80. Relationship between macrophages, Langerhans cells, reticular cells, and dendritic cells in lymphoid and hematopoietic organs. J Exp Med, 158, 1522-36.

HUME, D. A., ROSS, I. L., HIMES, S. R., SASMONO, R. T., WELLS, C. A. \& RAVASI, T. (2002) The mononuclear phagocyte system revisited. J Leukoc Biol, 72, 621-7.

HUO, Y., SCHOBER, A., FORLOW, S. B., SMITH, D. F., HYMAN, M. C., JUNG, S., LITTMAN, D. R., WEBER, C. \& LEY, K. (2003) Circulating activated platelets exacerbate atherosclerosis in mice deficient in apolipoprotein E. Nat Med, 9, 61-7.

HUYNH, M. L., FADOK, V. A. \& HENSON, P. M. (2002) Phosphatidylserinedependent ingestion of apoptotic cells promotes TGF-betal secretion and the resolution of inflammation. J Clin Invest, 109, 41-50.

ICHIDA, K., HOSOYAMADA, M., HISATOME, I., ENOMOTO, A., HIKITA, M., ENDOU, H. \& HOSOYA, T. (2004) Clinical and molecular analysis of patients with renal hypouricemia in Japan-influence of URAT1 gene on urinary urate excretion. J Am Soc Nephrol, 15, 164-73. 
JACOB, R. A., SPINOZZI, G. M., SIMON, V. A., KELLEY, D. S., PRIOR, R. L., HESS-PIERCE, B. \& KADER, A. A. (2003) Consumption of cherries lowers plasma urate in healthy women. J Nutr, 133, 1826-9.

JANSEN, B. J. M. \& DE GROOT, A. (2004) Occurrence, biological activity and synthesis of drimane sesquiterpenoids. Natural Produc Reports, 449-477.

JARAMILLO, M., GODBOUT, M., NACCACHE, P. H. \& OLIVIER, M. (2004a) Signaling events involved in macrophage chemokine expression in response to monosodium urate crystals. J Biol Chem, 279, 52797-805.

JARAMILLO, M., NACCACHE, P. H. \& OLIVIER, M. (2004b) Monosodium urate crystals synergize with IFN-gamma to generate macrophage nitric oxide: involvement of extracellular signal-regulated kinase 1/2 and NF-kappa B. $J$ Immunol, 172, 5734-42.

JOHNSON, R. J. \& RIDEOUT, B. A. (2004) Uric acid and diet--insights into the epidemic of cardiovascular disease. $N$ Engl J Med, 350, 1071-3.

JOHNSON, Z., PROUDFOOT, A. E. \& HANDEL, T. M. (2005) Interaction of chemokines and glycosaminoglycans: a new twist in the regulation of chemokine function with opportunities for therapeutic intervention. Cytokine Growth Factor Rev, 16, 625-36.

JORDAN, K. M., CAMERON, J. S., SNAITH, M., ZHANG, W., DOHERTY, M., SECKL, J., HINGORANI, A., JAQUES, R. \& NUKI, G. (2007) British Society for Rheumatology and British Health Professionals in Rheumatology guideline for the management of gout. Rheumatology (Oxford), 46, 1372-4.

KANSAS, G. S. (1996) Selectins and their ligands: current concepts and controversies. Blood, 88, 3259-87.

KERR, M. A. \& STOCKS, S. C. (1992) The role of CD15-(Le(X))-related carbohydrates in neutrophil adhesion. Histochem J, 24, 811-26.

KETTRITZ, R., FALK, R. J., JENNETTE, J. C. \& GAIDO, M. L. (1997) Neutrophil superoxide release is required for spontaneous and FMLP-mediated but not for TNF alpha-mediated apoptosis. J Am Soc Nephrol, 8, 1091-100.

KETTRITZ, R., GAIDO, M. L., HALLER, H., LUFT, F. C., JENNETTE, C. J. \& FALK, R. J. (1998) Interleukin-8 delays spontaneous and tumor necrosis factor-alpha-mediated apoptosis of human neutrophils. Kidney Int, 53, 84-91.

KHARAZMI, A., NIELSEN, H., RECHNITZER, C. \& BENDTZEN, K. (1989) Interleukin 6 primes human neutrophil and monocyte oxidative burst response. Immunol Lett, 21, 177-84.

KLEMP, P., STANSFIELD, S. A., CASTLE, B. \& ROBERTSON, M. C. (1997) Gout is on the increase in New Zealand. Ann Rheum Dis, 56, 22-6.

KNUDSEN, E., IVERSEN, P. O., VAN ROOIJEN, N. \& BENESTAD, H. B. (2002) Macrophage-dependent regulation of neutrophil mobilization and chemotaxis during development of sterile peritonitis in the rat. Eur J Haematol, 69, 28496.

KROGER, H., KLEWER, M., GRATZ, R., DIETRICH, A., EHRLICH, W., ALTRICHTER, S., KURPISZ, M. \& MIESEL, R. (1996) Influence of diet free of NAD-precursors on acetaminophen hepatotoxicity in mice. Gen Pharmacol, 27, 79-82.

KRUSKAL, B. A., SASTRY, K., WARNER, A. B., MATHIEU, C. E. \& EZEKOWITZ, R. A. (1992) Phagocytic chimeric receptors require both transmembrane and cytoplasmic domains from the mannose receptor. $J$ Exp Med, 176, 1673-80. 
KUBO, I., FUJITA, K. \& LEE, S. H. (2001) Antifungal mechanism of polygodial. $J$ Agric Food Chem, 49, 1607-11.

KUBO, I. \& GANJIAN, I. (1981) Insect antifeedant terpenes, hot-tasting to humans. Experientia, 37, 1063-4.

KUSHNER, I. \& SOMERVILLE, J. A. (1971) Permeability of human synovial membrane to plasma proteins. Relationship to molecular size and inflammation. Arthritis Rheum, 14, 560-70.

LAMBETH, J. D. (2004) NOX enzymes and the biology of reactive oxygen. Nat Rev Immunol, 4, 181-9.

LANDIS, R. C., YAGNIK, D. R., FLOREY, O., PHILIPPIDIS, P., EMONS, V., MASON, J. C. \& HASKARD, D. O. (2002) Safe disposal of inflammatory monosodium urate monohydrate crystals by differentiated macrophages. Arthritis Rheum, 46, 3026-33.

LAUDANNA, C., KIM, J. Y., CONSTANTIN, G. \& BUTCHER, E. (2002) Rapid leukocyte integrin activation by chemokines. Immunol Rev, 186, 37-46.

LEE, J., CACALANO, G., CAMERATO, T., TOY, K., MOORE, M. W. \& WOOD, W. I. (1995) Chemokine binding and activities mediated by the mouse IL-8 receptor. J Immunol, 155, 2158-64.

LEENEN, P. J. \& CAMPBELL, P. A. (1993) Macrophages and Related Cells. IN HORTON, M. A. (Ed.) Blood Cell Biochemistry. New York, Plenum Press.

LENNANE, G. A., ROSE, B. S. \& ISDALE, I. C. (1960) Gout in the Maori. Ann Rheum Dis, 19, 120-5.

LI, S., SANNA, S., MASCHIO, A., BUSONERO, F., USALA, G., MULAS, A., LAI, S., DEI, M., ORRU, M., ALBAI, G., BANDINELli, S., SCHLESSINGER, D., LAKATTA, E., SCUTERI, A., NAJJAR, S. S., GURALNIK, J., NAITZA, S., CRISPONI, L., CAO, A., ABECASIS, G., FERRUCCI, L., UDA, M., CHEN, W. M. \& NAGARAJA, R. (2007) The GLUT9 gene is associated with serum uric acid levels in Sardinia and Chianti cohorts. PLoS Genet, 3, e194.

LI, T., WALSH, J. R., GHISHAN, F. K. \& BAI, L. (2004) Molecular cloning and characterization of a human urate transporter (hURAT1) gene promoter. Biochim Biophys Acta, 1681, 53-8.

LICHANSKA, A. M. \& HUME, D. A. (2000) Origins and functions of phagocytes in the embryo. Exp Hematol, 28, 601-11.

LIOTE, F., PRUDHOMMEAUX, F., SCHILTZ, C., CHAMPY, R., HERBELIN, A., ORTIZ-BRAVO, E. \& BARDIN, T. (1996) Inhibition and prevention of monosodium urate monohydrate crystal-induced acute inflammation in vivo by transforming growth factor beta1. Arthritis Rheum, 39, 1192-8.

LIU-BRYAN, R., SCOTT, P., SYDLASKE, A., ROSE, D. M. \& TERKELTAUB, R. (2005) Innate immunity conferred by Toll-like receptors 2 and 4 and myeloid differentiation factor 88 expression is pivotal to monosodium urate monohydrate crystal-induced inflammation. Arthritis Rheum, 52, 2936-46.

LOU, O., ALCAIDE, P., LUSCINSKAS, F. W. \& MULLER, W. A. (2007) CD99 is a key mediator of the transendothelial migration of neutrophils. J Immunol, 178, 1136-43.

LU, B., RUTLEDGE, B. J., GU, L., FIORILLO, J., LUKACS, N. W., KUNKEL, S. L., NORTH, R., GERARD, C. \& ROLLINS, B. J. (1998) Abnormalities in monocyte recruitment and cytokine expression in monocyte chemoattractant protein 1-deficient mice. J Exp Med, 187, 601-8. 
MACLEAN-FLETCHER, S. \& POLLARD, T. D. (1980) Mechanism of action of cytochalasin B on actin. Cell, 20, 329-41.

MAMDOUH, Z., CHEN, X., PIERINI, L. M., MAXFIELD, F. R. \& MULLER, W. A. (2003) Targeted recycling of PECAM from endothelial surface-connected compartments during diapedesis. Nature, 421, 748-53.

MARIATHASAN, S., NEWTON, K., MONACK, D. M., VUCIC, D., FRENCH, D. M., LEE, W. P., ROOSE-GIRMA, M., ERICKSON, S. \& DIXIT, V. M. (2004) Differential activation of the inflammasome by caspase-1 adaptors ASC and Ipaf. Nature, 430, 213-8.

MARSHALL, B. T., LONG, M., PIPER, J. W., YAGO, T., MCEVER, R. P. \& ZHU, C. (2003) Direct observation of catch bonds involving cell-adhesion molecules. Nature, 423, 190-3.

MARTINON, F., BURNS, K. \& TSCHOPP, J. (2002) The inflammasome: a molecular platform triggering activation of inflammatory caspases and processing of proIL-beta. Mol Cell, 10, 417-26.

MARTINON, F., PETRILli, V., MAYOR, A., TARDIVEL, A. \& TSCHOPP, J. (2006) Gout-associated uric acid crystals activate the NALP3 inflammasome. Nature, 440, 237-41.

MARTINON, F. \& TSCHOPP, J. (2005) NLRs join TLRs as innate sensors of pathogens. Trends Immunol, 26, 447-54.

MATZINGER, P. (1994) Tolerance, danger, and the extended family. Annu Rev Immunol, 12, 991-1045.

MAZZALI, M., HUGHES, J., KIM, Y. G., JEFFERSON, J. A., KANG, D. H., GORDON, K. L., LAN, H. Y., KIVLIGHN, S. \& JOHNSON, R. J. (2001) Elevated uric acid increases blood pressure in the rat by a novel crystalindependent mechanism. Hypertension, 38, 1101-6.

MCCALliON, R. F., COLE, A. L., WALKER, J. R., BLUNT, J. W. \& MUNRO, M. H. (1982) Antibiotic substances from New Zealand plants. II. Polygodial, an anti-Candida agent from Pseudowintera colorata. Planta Med, 44, 134-8.

MCCARTY, D. J., JR., PHELPS, P. \& PYENSON, J. (1966) Crystal-induced inflammation in canine joints. I. An experimental model with quantification of the host response. $J$ Exp Med, 124, 99-114.

MCEVER, R. P. \& CUMMINGS, R. D. (1997) Role of PSGL-1 binding to selectins in leukocyte recruitment. J Clin Invest, 100, S97-103.

MELNICOFF, M. J., HORAN, P. K. \& MORAHAN, P. S. (1989) Kinetics of changes in peritoneal cell populations following acute inflammation. Cell Immunol, 118, 178-91.

MENDES, G. L., SANTOS, A. R., MALHEIROS, A., FILHO, V. C., YUNES, R. A. \& CALIXTO, J. B. (2000) Assessment of mechanisms involved in antinociception caused by sesquiterpene polygodial. $J$ Pharmacol Exp Ther, 292, 164-72.

MESSERLI, F. H., FROHLICH, E. D., DRESLINSKI, G. R., SUAREZ, D. H. \& ARISTIMUNO, G. G. (1980) Serum uric acid in essential hypertension: an indicator of renal vascular involvement. Ann Intern Med, 93, 817-21.

MIESEL, R., KURPISZ, M. \& KROGER, H. (1996) Suppression of inflammatory arthritis by simultaneous inhibition of nitric oxide synthase and NADPH oxidase. Free Radic Biol Med, 20, 75-81.

MIKULS, T. R., MACLEAN, C. H., OLIVIERI, J., PATINO, F., ALLISON, J. J., FARRAR, J. T., BILKER, W. B. \& SAAG, K. G. (2004) Quality of care indicators for gout management. Arthritis Rheum, 50, 937-43. 
MILLAN, J. \& RIDLEY, A. J. (2005) Rho GTPases and leucocyte-induced endothelial remodelling. Biochem J, 385, 329-37.

MORTON, J. F. (1981) IN THOMAS, C. C. (Ed.) Atlas of Medicinal Plants in Meddle America-Bahamas to Yucatan. Springfiled Thomas.

MULLER, J. \& YOSHIDA, T. (1995) Interaction of murine peritoneal leukocytes and mesothelial cells: in vitro model system to survey cellular events on serosal membranes during inflammation. Clin Immunol Immunopathol, 75, 231-8.

MULLER, W. A. (2003) Leukocyte-endothelial-cell interactions in leukocyte transmigration and the inflammatory response. Trends Immunol, 24, 327-34.

MURAKAMI, Y., AKAHOSHI, T., HAYASHI, I., ENDO, H., KAWAI, S., INOUE, M., KONDO, H. \& KITASATO, H. (2006) Induction of triggering receptor expressed on myeloid cells 1 in murine resident peritoneal macrophages by monosodium urate monohydrate crystals. Arthritis Rheum, $54,455-62$.

MURRAY, A. W. (1971) The biological significance of purine salvage. Annu Rev Biochem, 40, 811-26.

NACCACHE, P. H., BOURGoIn, S., PlAnte, E., ROBERGE, C. J., DE MEDICIS, R., LUSSIER, A. \& POUBELLE, P. E. (1993) Crystal-induced neutrophil activation. II. Evidence for the activation of a phosphatidylcholine-specific phospholipase D. Arthritis Rheum, 36, 117-25.

NACCACHE, P. H., GRIMARD, M., ROBERGE, C. J., GILBERT, C., LUSSIER, A., DE MEDICIS, R. \& POUBELLE, P. E. (1991) Crystal-induced neutrophil activation. I. Initiation and modulation of calcium mobilization and superoxide production by microcrystals. Arthritis Rheum, 34, 333-42.

NAKAYAMA, D. A., BARTHELEMY, C., CARRERA, G., LIGHTFOOT, R. W., JR. \& WORTMANN, R. L. (1984) Tophaceous gout: a clinical and radiographic assessment. Arthritis Rheum, 27, 468-71.

NAUSEEF, W. M., VOLPP, B. D., MCCORMICK, S., LEIDAL, K. G. \& CLARK, R. A. (1991) Assembly of the neutrophil respiratory burst oxidase. Protein kinase $\mathrm{C}$ promotes cytoskeletal and membrane association of cytosolic oxidase components. J Biol Chem, 266, 5911-7.

NELSON, D. S. \& BOYDEN, S. V. (1963) The loss of macrophages from peritoneal exudates following the injection of antigens into guinea-pigs with delayedtype hypersensitivity. Immunology, 6, 264-75.

NICHOLLS, A., SNAITH, M. L. \& SCOTT, J. T. (1973) Effect of oestrogen therapy on plasma and urinary levels of uric acid. Br Med $J, 1,449-51$.

NiCOlETTI, I., Migliorati, G., PAGLiACCI, M. C., GRIGNANI, F. \& RICCARDI, C. (1991) A rapid and simple method for measuring thymocyte apoptosis by propidium iodide staining and flow cytometry. J Immunol Methods, 139, 271-9.

NOURSHARGH, S., KROMBACH, F. \& DEJANA, E. (2006) The role of JAM-A and PECAM-1 in modulating leukocyte infiltration in inflamed and ischemic tissues. J Leukoc Biol, 80, 714-8.

NUKI, G. \& SIMKIN, P. A. (2006) A concise history of gout and hyperuricemia and their treatment. Arthritis Res Ther, 8 Suppl 1, S1.

ORTIZ-BRAVO, E., SIECK, M. S. \& SCHUMACHER, H. R., JR. (1993) Changes in the proteins coating monosodium urate crystals during active and subsiding inflammation. Immunogold studies of synovial fluid from patients 
with gout and of fluid obtained using the rat subcutaneous air pouch model. Arthritis Rheum, 36, 1274-85.

OZINSKY, A., UNDERHILL, D. M., FONTENOT, J. D., HAJJAR, A. M., SMITH, K. D., WILSON, C. B., SCHROEDER, L. \& ADEREM, A. (2000) The repertoire for pattern recognition of pathogens by the innate immune system is defined by cooperation between toll-like receptors. Proc Natl Acad Sci U S $A, 97,13766-71$.

PACHER, P., NIVOROZHKIN, A. \& SZABO, C. (2006) Therapeutic effects of xanthine oxidase inhibitors: renaissance half a century after the discovery of allopurinol. Pharmacol Rev, 58, 87-114.

PASCUAL, E. (1991) Persistence of monosodium urate crystals and low-grade inflammation in the synovial fluid of patients with untreated gout. Arthritis Rheum, 34, 141-5.

PASCUAL, E., BATlle-GUALDA, E., MARTineZ, A., ROSAS, J. \& VELA, P. (1999) Synovial fluid analysis for diagnosis of intercritical gout. Ann Intern Med, 131, 756-9.

PASCUAL, E. \& JOVANI, V. (1995) A quantitative study of the phagocytosis of urate crystals in the synovial fluid of asymptomatic joints of patients with gout. Br J Rheumatol, 34, 724-6.

PASSLICK, B., FLIEGER, D. \& ZIEGLER-HEITBROCK, H. W. (1989) Identification and characterization of a novel monocyte subpopulation in human peripheral blood. Blood, 74, 2527-34.

PETRI, B. \& BIXEL, M. G. (2006) Molecular events during leukocyte diapedesis. FEBS J, 273, 4399-407.

PHELPS, P. \& MCCARTY, D. J., JR. (1966) Crystal-induced inflammation in canine joints. II. Importance of polymorphonuclear leukocytes. J Exp Med, 124, 115-26.

PHILliPSON, M., HEIT, B., COLARUSSO, P., LIU, L., BALLANTYNE, C. M. \& KUBES, P. (2006) Intraluminal crawling of neutrophils to emigration sites: a molecularly distinct process from adhesion in the recruitment cascade. $J \operatorname{Exp}$ Med, 203, 2569-75.

POOR, G. \& MITUSZOVA, M. (2003) Crystal-related arthropathies. IN HOCHBERG, M. C., SILMAN, A. J., SMOLEN, J. S., WEINBLATT, M. E. \& WEISMAN, M. H. (Eds.) Rheumatology. Third ed. London, Mosby.

PORTER, R. \& ROUSSEAU, G. S. (1998) Gout: The Patrician Malady, New Haven, Yale University Press.

PROCTOR, P. (1970) Similar functions of uric acid and ascorbate in man? Nature, $228,868$.

PUIG, J. G. \& FOX, I. H. (1984) Ethanol-induced activation of adenine nucleotide turnover. Evidence for a role of acetate. $J$ Clin Invest, 74, 936-41.

PUIG, J. G. \& RUILOPE, L. M. (1999) Uric acid as a cardiovascular risk factor in arterial hypertension. J Hypertens, 17, 869-72.

RAIVIO, K. O., BECKER, A., MEYER, L. J., GREENE, M. L., NUKI, G. \& SEEGMILLER, J. E. (1975) Stimulation of human purine synthesis de novo by fructose infusion. Metabolism, 24, 861-9.

RANDOLPH, G. J. \& FURIE, M. B. (1995) A soluble gradient of endogenous monocyte chemoattractant protein-1 promotes the transendothelial migration of monocytes in vitro. J Immunol, 155, 3610-8. 
RANDOLPH, G. J., INABA, K., ROBBIANI, D. F., STEINMAN, R. M. \& MULLER, W. A. (1999) Differentiation of phagocytic monocytes into lymph node dendritic cells in vivo. Immunity, 11, 753-61.

REINHARDT, P. H., NACCACHE, P. H., POUBELLE, P. E., DE MEDICIS, R., KEHRLI, M. E., JR. \& KUBES, P. (1996) Monosodium urate crystals promote neutrophil adhesion via a CD18-independent and selectinindependent mechanism. Am J Physiol, 270, C31-9.

RICHARDS, P. J., WILLIAMS, A. S., GOODFELLOW, R. M. \& WILLIAMS, B. D. (1999) Liposomal clodronate eliminates synovial macrophages, reduces inflammation and ameliorates joint destruction in antigen-induced arthritis. Rheumatology (Oxford), 38, 818-25.

RIESE, J., DENZEL, C., ZOWE, M., MEHLER, C., HOHENBERGER, W. \& HAUPT, W. (1999) Secretion of IL-6, monocyte chemoattractant protein-1, macrophage inflammatory protein-1alpha, and TNFalpha by cultured intact human peritoneum. Eur Surg Res, 31, 281-8.

ROSE, D. M., SYDLASKE, A. D., AGHA-BABAKHANI, A., JOHNSON, K. \& TERKELTAUB, R. (2006) Transglutaminase 2 limits murine peritoneal acute gout-like inflammation by regulating macrophage clearance of apoptotic neutrophils. Arthritis Rheum, 54, 3363-71.

ROUBENOFF, R. (1990) Gout and hyperuricemia. Rheum Dis Clin North Am, 16, 539-50.

ROZENBERG-ARSKA, M., SALTERS, M. E., VAN STRIJP, J. A., GEUZE, J. J. \& VERHOEF, J. (1985) Electron microscopic study of phagocytosis of Escherichia coli by human polymorphonuclear leukocytes. Infect Immun, 50, 852-9.

RUSSELL, I. J., MANSEN, C., KOLB, L. M. \& KOLB, W. P. (1982) Activation of the fifth component of human complement (C5) induced by monosodium urate crystals: C5 convertase assembly on the crystal surface. Clin Immunol Immunopathol, 24, 239-50.

RYCKMAN, C., GILBERT, C., DE MEDICIS, R., LUSSIER, A., VANDAL, K. \& TESSIER, P. A. (2004) Monosodium urate monohydrate crystals induce the release of the proinflammatory protein S100A8/A9 from neutrophils. $J$ Leukoc Biol, 76, 433-40.

RYCKMAN, C., MCCOLL, S. R., VANDAL, K., DE MEDICIS, R., LUSSIER, A., POUBELLE, P. E. \& TESSIER, P. A. (2003a) Role of S100A8 and S100A9 in neutrophil recruitment in response to monosodium urate monohydrate crystals in the air-pouch model of acute gouty arthritis. Arthritis Rheum, 48, 2310-20.

RYCKMAN, C., VANDAL, K., ROULEAU, P., TALBOT, M. \& TESSIER, P. A. (2003b) Proinflammatory activities of S100: proteins S100A8, S100A9, and S100A8/A9 induce neutrophil chemotaxis and adhesion. J Immunol, 170, 3233-42.

SAAG, K. G. \& CHOI, H. (2006) Epidemiology, risk factors, and lifestyle modifications for gout. Arthritis Res Ther, 8 Suppl 1, S2.

SALLUSTO, F. \& LANZAVECCHIA, A. (1994) Efficient presentation of soluble antigen by cultured human dendritic cells is maintained by granulocyte/macrophage colony-stimulating factor plus interleukin 4 and downregulated by tumor necrosis factor alpha. J Exp Med, 179, 1109-18.

SANCHEZ-TORRES, C., GARCIA-ROMO, G. S., CORNEJO-CORTES, M. A., RIVAS-CARVALHO, A. \& SANCHEZ-SCHMITZ, G. (2001) CD16+ and 
CD16- human blood monocyte subsets differentiate in vitro to dendritic cells with different abilities to stimulate CD4+ T cells. Int Immunol, 13, 1571-81.

SARAWATE, C. A., BREWER, K. K., YANG, W., PATEL, P. A., SCHUMACHER, H. R., SAAG, K. G. \& BAKST, A. W. (2006) Gout medication treatment patterns and adherence to standards of care from a managed care perspective. Mayo Clin Proc, 81, 925-34.

SASMONO, R. T., OCEANDY, D., POLLARD, J. W., TONG, W., PAVLI, P., WAINWRIGHT, B. J., OSTROWSKI, M. C., HIMES, S. R. \& HUME, D. A. (2003) A macrophage colony-stimulating factor receptor-green fluorescent protein transgene is expressed throughout the mononuclear phagocyte system of the mouse. Blood, 101, 1155-63.

SCHALLER, E., MACFARLANE, A. J., RUPEC, R. A., GORDON, S., MCKNIGHT, A. J. \& PFEFFER, K. (2002) Inactivation of the F4/80 glycoprotein in the mouse germ line. Mol Cell Biol, 22, 8035-43.

SCHENKEL, A. R., MAMDOUH, Z., CHEN, X., LIEBMAN, R. M. \& MULLER, W. A. (2002) CD99 plays a major role in the migration of monocytes through endothelial junctions. Nat Immunol, 3, 143-50.

SCHENKEL, A. R., MAMDOUH, Z. \& MULLER, W. A. (2004) Locomotion of monocytes on endothelium is a critical step during extravasation. Nat Immunol, 5, 393-400.

SCHILTZ, C., LIOTE, F., PRUDHOMMEAUX, F., MEUNIER, A., CHAMPY, R., CALLEBERT, J. \& BARDIN, T. (2002) Monosodium urate monohydrate crystal-induced inflammation in vivo: quantitative histomorphometric analysis of cellular events. Arthritis Rheum, 46, 1643-50.

SCHMID, K. \& MACNAIR, M. B. (1956) Characterization of the proteins of human synovial fluid in certain disease states. J Clin Invest, 35, 814-24.

SCHREINER, O., WANDEL, E., HIMMELSBACH, F., GALLE, P. R. \& MARKER-HERMANN, E. (2000) Reduced secretion of proinflammatory cytokines of monosodium urate crystal-stimulated monocytes in chronic renal failure: an explanation for infrequent gout episodes in chronic renal failure patients? Nephrol Dial Transplant, 15, 644-9.

SCHWARTZ, S. A. (2006) Disease of distinction. Explore (NY), 2, 515-9.

SCOTT, P., MA, H., VIRIYAKOSOL, S., TERKELTAUB, R. \& LIU-BRYAN, R. (2006) Engagement of CD14 mediates the inflammatory potential of monosodium urate crystals. J Immunol, 177, 6370-8.

SEGAL, A. W. (2005) How neutrophils kill microbes. Annu Rev Immunol, 23, 197223.

SEGAL, B. H., LETO, T. L., GALLIN, J. I., MALECH, H. L. \& HOLLAND, S. M. (2000) Genetic, biochemical, and clinical features of chronic granulomatous disease. Medicine (Baltimore), 79, 170-200.

SERBINA, N. V. \& PAMER, E. G. (2006) Monocyte emigration from bone marrow during bacterial infection requires signals mediated by chemokine receptor CCR2. Nat Immunol, 7, 311-7.

SHAMRI, R., GRABOVSKY, V., GAUGUET, J. M., FEIGELSON, S., MANEVICH, E., KOLANUS, W., ROBINSON, M. K., STAUNTON, D. E., VON ANDRIAN, U. H. \& ALON, R. (2005) Lymphocyte arrest requires instantaneous induction of an extended LFA-1 conformation mediated by endothelium-bound chemokines. Nat Immunol, 6, 497-506. 
SHAW, S. K., BAMBA, P. S., PERKINS, B. N. \& LUSCINSKAS, F. W. (2001) Real-time imaging of vascular endothelial-cadherin during leukocyte transmigration across endothelium. J Immunol, 167, 2323-30.

SHI, J., GILBERT, G. E., KOKUBO, Y. \& OHASHI, T. (2001) Role of the liver in regulating numbers of circulating neutrophils. Blood, 98, 1226-30.

SHI, Y., EVANS, J. E. \& ROCK, K. L. (2003) Molecular identification of a danger signal that alerts the immune system to dying cells. Nature, 425, 516-21.

SHI, Y. \& ROCK, K. L. (2002) Cell death releases endogenous adjuvants that selectively enhance immune surveillance of particulate antigens. Eur $J$ Immunol, 32, 155-62.

SHI, Y., ZHENG, W. \& ROCK, K. L. (2000) Cell injury releases endogenous adjuvants that stimulate cytotoxic T cell responses. Proc Natl Acad Sci U S $A, 97,14590-5$.

SHIMA, Y., TERUYA, K. \& OHTA, H. (2006) Association between intronic SNP in urate-anion exchanger gene, SLC22A12, and serum uric acid levels in Japanese. Life Sci, 79, 2234-7.

SHORTMAN, K. \& LIU, Y. J. (2002) Mouse and human dendritic cell subtypes. Nat Rev Immunol, 2, 151-61.

SIES, H. (1997) Oxidative stress: oxidants and antioxidants. Exp Physiol, 82, 291-5.

SIMCHOWITZ, L., ATKINSON, J. P. \& SPILBERG, I. (1982) Stimulation of the respiratory burst in human neutrophils by crystal phagocytosis. Arthritis Rheum, 25, 181-8.

SIXT, M., ENGELHARDT, B., PAUSCH, F., HALLMANN, R., WENDLER, O. \& SOROKIN, L. M. (2001) Endothelial cell laminin isoforms, laminins 8 and 10 , play decisive roles in $\mathrm{T}$ cell recruitment across the blood-brain barrier in experimental autoimmune encephalomyelitis. J Cell Biol, 153, 933-46.

SO, A., DE SMEDT, T., REVAZ, S. \& TSCHOPP, J. (2007) A pilot study of IL-1 inhibition by anakinra in acute gout. Arthritis Res Ther, 9, R28.

STARK, K., REINHARD, W., NEUREUTHER, K., WIEDMANN, S., SEDLACEK, K., BAESSLER, A., FISCHER, M., WEBER, S., KAESS, B., ERDMANN, J., SCHUNKERT, H. \& HENGSTENBERG, C. (2008) Association of common polymorphisms in GLUT9 gene with gout but not with coronary artery disease in a large case-control study. PLoS ONE, 3, e1948.

STEIN, M., KESHAV, S., HARRIS, N. \& GORDON, S. (1992) Interleukin 4 potently enhances murine macrophage mannose receptor activity: a marker of alternative immunologic macrophage activation. J Exp Med, 176, 287-92.

STERNLICHT, H. \& RINGEL, I. (1979) Colchicine inhibition of microtubule assembly via copolymer formation. J Biol Chem, 254, 10540-50.

STOUT, R. D. \& SUTTLES, J. (2004) Functional plasticity of macrophages: reversible adaptation to changing microenvironments. J Leukoc Biol, 76, 509-13.

STRIETER, R. M., KASAHARA, K., ALLEN, R. M., STANDIFORD, T. J., ROLFE, M. W., BECKER, F. S., CHENSUE, S. W. \& KUNKEL, S. L. (1992) Cytokine-induced neutrophil-derived interleukin-8. Am J Pathol, 141, 397-407.

SUllivan, G. W., CARPER, H. T., SUllivan, J. A., MURATA, T. \& MANDELL, G. L. (1989) Both recombinant interleukin-1 (beta) and purified human monocyte interleukin-1 prime human neutrophils for increased 
oxidative activity and promote neutrophil spreading. J Leukoc Biol, 45, 38995.

SUNDERKOTTER, C., NIKOLIC, T., DILlON, M. J., VAN ROOIJEN, N., STEHLING, M., DREVETS, D. A. \& LEENEN, P. J. (2004) Subpopulations of mouse blood monocytes differ in maturation stage and inflammatory response. J Immunol, 172, 4410-7.

SUSSMAN, J. S., BROZENA, S. C., SKOP, N., KORECKA, M. \& SHAW, L. M. (2004) Accidental intravenous colchicine poisoning. Ther Drug Monit, 26, 688-92.

SWEET, D. H., CHAN, L. M., WALDEN, R., YANG, X. P., MILlER, D. S. \& PRITCHARD, J. B. (2003) Organic anion transporter 3 (Slc22a8) is a dicarboxylate exchanger indirectly coupled to the $\mathrm{Na}+$ gradient. Am J Physiol Renal Physiol, 284, F763-9.

SWEET, M. J. \& HUME, D. A. (2003) CSF-1 as a regulator of macrophage activation and immune responses. Arch Immunol Ther Exp (Warsz), 51, 16977.

SYDENHAM, T. (1683) Tractatus de Podagra et Hydrope, London, G Kettilby.

TAN, A. S. \& BERRIDGE, M. V. (2000) Superoxide produced by activated neutrophils efficiently reduces the tetrazolium salt, WST-1 to produce a soluble formazan: a simple colorimetric assay for measuring respiratory burst activation and for screening anti-inflammatory agents. J Immunol Methods, 238, 59-68.

TANIGUCHI, A. \& KAMATANI, N. (2008) Control of renal uric acid excretion and gout. Curr Opin Rheumatol, 20, 192-7.

TERKELTAUB, R., BAIRD, S., SEARS, P., SANTIAGO, R. \& BOISVERT, W. (1998) The murine homolog of the interleukin-8 receptor CXCR-2 is essential for the occurrence of neutrophilic inflammation in the air pouch model of acute urate crystal-induced gouty synovitis. Arthritis Rheum, 41, 900-9.

TERKELTAUB, R., BUSHINSKY, D. A. \& BECKER, M. A. (2006) Recent developments in our understanding of the renal basis of hyperuricemia and the development of novel antihyperuricemic therapeutics. Arthritis Res Ther, 8 Suppl 1, S4.

TERKELTAUB, R., CURTISS, L. K., TENNER, A. J. \& GINSBERG, M. H. (1984) Lipoproteins containing apoprotein $\mathrm{B}$ are a major regulator of neutrophil responses to monosodium urate crystals. $J$ Clin Invest, 73, 1719-30.

TERKElTAUB, R., ZACHARIAE, C., SANTORO, D., MARTIN, J., PEVERI, P. \& MATSUSHIMA, K. (1991) Monocyte-derived neutrophil chemotactic factor/interleukin-8 is a potential mediator of crystal-induced inflammation. Arthritis Rheum, 34, 894-903.

THOMAS-ECKER, S., LINDECKE, A., HATZMANN, W., KALTSCHMIDT, C., ZANKER, K. S. \& DITTMAR, T. (2007) Alteration in the gene expression pattern of primary monocytes after adhesion to endothelial cells. Proc Natl Acad Sci U S A, 104, 5539-44.

TJELlE, T. E., LOVDAL, T. \& BERG, T. (2000) Phagosome dynamics and function. Bioessays, 22, 255-63.

TRAMONTINI, N., HUBER, C., LIU-BRYAN, R., TERKELTAUB, R. A. \& KILGORE, K. S. (2004) Central role of complement membrane attack complex in monosodium urate crystal-induced neutrophilic rabbit knee synovitis. Arthritis Rheum, 50, 2633-9. 
TUDAN, C., FONG, D., DURONIO, V., BURT, H. M. \& JACKSON, J. K. (2000) The inhibition of spontaneous and tumor necrosis factor-alpha induced neutrophil apoptosis by crystals of calcium pyrophosphate dihydrate and monosodium urate monohydrate. J Rheumatol, 27, 2463-72.

UDAGAWA, N., TAKAHASHI, N., AKATSU, T., TANAKA, H., SASAKI, T., NISHIHARA, T., KOGA, T., MARTIN, T. J. \& SUDA, T. (1990) Origin of osteoclasts: mature monocytes and macrophages are capable of differentiating into osteoclasts under a suitable microenvironment prepared by bone marrow-derived stromal cells. Proc Natl Acad Sci US A, 87, 7260-4.

VAN FURTH, R. \& COHN, Z. A. (1968) The origin and kinetics of mononuclear phagocytes. J Exp Med, 128, 415-35.

VAN ROOIJEN, N., SANDERS, A. \& VAN DEN BERG, T. K. (1996) Apoptosis of macrophages induced by liposome-mediated intracellular delivery of clodronate and propamidine. J Immunol Methods, 193, 93-9.

VAZQUEZ-MELLADO, J., JIMENEZ-VACA, A. L., CUEVAS-COVARRUBIAS, S., ALVARADO-ROMANO, V., POZO-MOLINA, G. \& BURGOSVARGAS, R. (2007) Molecular analysis of the SLC22A12 (URAT1) gene in patients with primary gout. Rheumatology (Oxford), 46, 215-9.

VERMES, I., HAANEN, C., STEFFENS-NAKKEN, H. \& REUTELINGSPERGER, C. (1995) A novel assay for apoptosis. Flow cytometric detection of phosphatidylserine expression on early apoptotic cells using fluorescein labelled Annexin V. J Immunol Methods, 184, 39-51.

VESTWEBER, D. (2002) Regulation of endothelial cell contacts during leukocyte extravasation. Curr Opin Cell Biol, 14, 587-93.

VON HUNDELSHAUSEN, P., WEBER, K. S., HUO, Y., PROUDFOOT, A. E., NELSON, P. J., LEY, K. \& WEBER, C. (2001) RANTES deposition by platelets triggers monocyte arrest on inflamed and atherosclerotic endothelium. Circulation, 103, 1772-7.

WALLACE, K. L., RIEDEL, A. A., JOSEPH-RIDGE, N. \& WORTMANN, R. (2004) Increasing prevalence of gout and hyperuricemia over 10 years among older adults in a managed care population. $J$ Rheumatol, 31, 1582-7.

WANG, S., DANGERFIELD, J. P., YOUNG, R. E. \& NOURSHARGH, S. (2005) PECAM-1, alpha6 integrins and neutrophil elastase cooperate in mediating neutrophil transmigration. J Cell Sci, 118, 2067-76.

WANG, S., VOISIN, M. B., LARBI, K. Y., DANGERFIELD, J., SCHEIERMANN, C., TRAN, M., MAXWELL, P. H., SOROKIN, L. \& NOURSHARGH, S. (2006) Venular basement membranes contain specific matrix protein low expression regions that act as exit points for emigrating neutrophils. $J$ Exp Med, 203, 1519-32.

WARREN, M. K. \& VOGEL, S. N. (1985) Bone marrow-derived macrophages: development and regulation of differentiation markers by colony-stimulating factor and interferons. J Immunol, 134, 982-9.

WATANABE, S., KANG, D. H., FENG, L., NAKAGAWA, T., KANELLIS, J., LAN, H., MAZZALI, M. \& JOHNSON, R. J. (2002) Uric acid, hominoid evolution, and the pathogenesis of salt-sensitivity. Hypertension, 40, 355-60.

WEBER, C., BELGE, K. U., VON HUNDELSHAUSEN, P., DRAUDE, G., STEPPICH, B., MACK, M., FRANKENBERGER, M., WEBER, K. S. \& ZIEGLER-HEITBROCK, H. W. (2000) Differential chemokine receptor expression and function in human monocyte subpopulations. J Leukoc Biol, 67, 699-704. 
WEISSMANN, G. \& RITA, G. A. (1972) Molecular basis of gouty inflammation: interaction of monosodium urate crystals with lysosomes and liposomes. Nature, 240, 167-172.

WU, X. W., LEE, C. C., MUZNY, D. M. \& CASKEY, C. T. (1989) Urate oxidase: primary structure and evolutionary implications. Proc Natl Acad Sci U S A, 86, 9412-6.

WU, X. W., MUZNY, D. M., LEE, C. C. \& CASKEY, C. T. (1992) Two independent mutational events in the loss of urate oxidase during hominoid evolution. J Mol Evol, 34, 78-84.

YAGNIK, D. R., EVANS, B. J., FLOREY, O., MASON, J. C., LANDIS, R. C. \& HASKARD, D. O. (2004) Macrophage release of transforming growth factor betal during resolution of monosodium urate monohydrate crystal-induced inflammation. Arthritis Rheum, 50, 2273-80.

YAGNIK, D. R., HILLYER, P., MARSHALL, D., SMYTHE, C. D., KRAUSZ, T., HASKARD, D. O. \& LANDIS, R. C. (2000) Noninflammatory phagocytosis of monosodium urate monohydrate crystals by mouse macrophages. Implications for the control of joint inflammation in gout. Arthritis Rheum, 43, 1779-89.

YAGO, T., ZARNITSYNA, V. I., KLOPOCKI, A. G., MCEVER, R. P. \& ZHU, C. (2007) Transport governs flow-enhanced cell tethering through L-selectin at threshold shear. Biophys J, 92, 330-42.

ZHOU, L. J. \& TEDDER, T. F. (1996) CD14+ blood monocytes can differentiate into functionally mature CD83+ dendritic cells. Proc Natl Acad Sci U S A, 93, 2588-92. 


\section{(̇) invitrogen}

DB. M

Dulbecco's Phosphate-Buffered Saline (D-PBS) (1X) liquid

Contains calcium and magnesium.

Catalog Number(s): $14040075, \underline{14040083}, \underline{14040091}, \underline{14040117}, \underline{14040133}, \underline{14040141}, \underline{14040158,14040166}$ $14040174,14040182,14040216$

\begin{tabular}{|l|r|r|r|}
\hline COMPONENTS & Molecular Weight & Concentration (mg/L) & Molarity \\
\hline Inorganic Salts & & 100 & 0.900901 \\
\hline Calcium Chloride (CaCl2) (anhyd.) & 111 & 100 & 0.492611 \\
\hline Magnesium Chloride (MgCl2-6H2O) & 203 & 200 & 2.666667 \\
\hline Potassium Chloride (KCl) & 75 & 200 & 1.470588 \\
\hline Potassium Phosphate monobasic (KH2PO4) & 136 & 8000 & 137.93103 \\
\hline Sodium Chloride (NaCl) & 58 & 2160 & 8.059702 \\
\hline Sodium Phosphate dibasic (Na2HPO4-7H2O) & 268 & &
\end{tabular}

Related Products 


\section{in invitrogen}

Technical Resources - Media Formulations

Hanks' Balanced Salt Solution (HBSS) (1X) liquid

Contains calcium and magnesium.

Balanced salt solutions are based on the physiological saline first developed by Ringer. They are composed of inorganic salts and may be supplemented with glucose. Balanced salt solutions have been designed to maintain cells in a viable state for short periods of time rather than to promote growth. Balanced salt solutions are used

for washing tissues and cells and as diluents for treating cells and tissues with various agents, while maintaining a physiological pH and osmotic pressure. Hank's Balanced Salt Solution is buffered with phosphate. Therefore

the solution will maintain its physiological pH at atmospheric conditions. For this reason it is the primary solution

used in enzymatic treatments of cells and tissue and the final rinse of cells prior to the suspension of the cells in

a complete growth medium.

Catalog Number(s): $\underline{24020018}, \underline{24020034}, \underline{24020059}, \underline{24020067}, \underline{24020075}, \underline{24020083}, \underline{24020091}, \underline{24020109}$

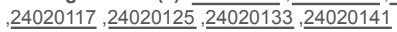

\begin{tabular}{|c|c|c|c|}
\hline COMPONENTS & $\begin{array}{r}\text { Molecular } \\
\text { Weight }\end{array}$ & $\begin{array}{r}\begin{array}{r}\text { Concentration } \\
(\mathrm{mg} / \mathrm{L})\end{array} \\
\end{array}$ & Molarity \\
\hline \multicolumn{4}{|l|}{ Inorganic Salts } \\
\hline Calcium Chloride (CaCl2) (anhyd.) & 111 & 140 & 1.261261 \\
\hline Magnesium Chloride (MgCl2-6H2O) & 203 & 100 & 0.492611 \\
\hline Magnesium Sulfate (MgSO4-7H2O) & 246 & 100 & 0.406504 \\
\hline Potassium Chloride (KCl) & 75 & 400 & 5.333334 \\
\hline Potassium Phosphate monobasic (KH2PO4) & 136 & 60 & 0.441176 \\
\hline Sodium Bicarbonate ( $\mathrm{NaHCO} 3$ ) & 84 & 350 & 4.166666 \\
\hline Sodium Chloride ( $\mathrm{NaCl}$ ) & 58 & 8000 & 137.93103 \\
\hline $\begin{array}{l}\text { Sodium Phosphate dibasic (Na2HPO4) } \\
\text { anhydrous }\end{array}$ & 142 & 48 & 0.338028 \\
\hline \multicolumn{4}{|l|}{ Other Components } \\
\hline D-Glucose (Dextrose) & 180 & 1000 & 5.555555 \\
\hline Phenol Red & 376.4 & 10 & 0.026567 \\
\hline
\end{tabular}

$\underline{\text { Related Products }}$ 


\section{๕̊ invitrogen}

Technical Resources - Media Formulations

Advanced RPMI Medium 1640 (1X) liquid

Contains 2,000 mg/L D-glucose, non-essential amino acids (NEAA), and $110 \mathrm{mg} / \mathrm{L}$ sodium pyruvate, but no L-glutamine.

RPMI (Roswell Park Memorial Institute) Media 1640 are enriched formulations with extensive applications for mammalian cells. They were originally formulated for suspension cultures or monolayer cultures of human

leukemia cells.

Catalog Number(s): 12633012,12633020

\begin{tabular}{|c|c|c|c|}
\hline COMPONENTS & $\begin{array}{r}\text { Molecular } \\
\text { Weight }\end{array}$ & $\begin{array}{r}\text { Concentration } \\
\text { (mg/L) }\end{array}$ & Molarity \\
\hline \multicolumn{4}{|l|}{ Amino Acids } \\
\hline Glycine & 75 & 10 & 0.133333 \\
\hline L-Alanine & 89 & 8.9 & 0.1 \\
\hline L-Arginine & 174 & 200 & 1.149425 \\
\hline L-Asparagine & 132 & 50 & 0.378788 \\
\hline L-Aspartic acid & 133 & 20 & 0.150376 \\
\hline L-Cystine $2 \mathrm{HCl}$ & 313 & 65 & 0.207668 \\
\hline L-Glutamic Acid & 147 & 20 & 0.136054 \\
\hline L-Histidine & 155 & 15 & 0.096774 \\
\hline L-Hydroxyproline & 131 & 20 & 0.152672 \\
\hline L-Isoleucine & 131 & 50 & 0.381679 \\
\hline L-Leucine & 131 & 50 & 0.381679 \\
\hline L-Lysine hydrochloride & 146 & 40 & 0.273973 \\
\hline L-Methionine & 149 & 15 & 0.100671 \\
\hline L-Phenylalanine & 165 & 15 & 0.090909 \\
\hline L-Proline & 115 & 20 & 0.173913 \\
\hline L-Serine & 105 & 30 & 0.285714 \\
\hline L-Threonine & 119 & 20 & 0.168067 \\
\hline L-Tryptophan & 204 & 5 & 0.02451 \\
\hline L-Tyrosine disodium salt & 225 & 29 & 0.128889 \\
\hline L-Valine & 117 & 20 & 0.17094 \\
\hline \multicolumn{4}{|l|}{ Vitamins } \\
\hline Ascorbic Acid phosphate & 289.54 & 2.5 & 0.008634 \\
\hline Biotin & 244 & 0.2 & 0.00082 \\
\hline Choline chloride & 140 & 3 & 0.021429 \\
\hline D-Calcium pantothenate & 477 & 0.25 & 0.000524 \\
\hline Folic Acid & 441 & 1 & 0.002268 \\
\hline Niacinamide & 122 & 1 & 0.008197 \\
\hline Para-Aminobenzoic Acid & 137 & 1 & 0.007299 \\
\hline Pyridoxine hydrochloride & 206 & 1 & 0.004854 \\
\hline Riboflavin & 376 & 0.2 & 0.000532 \\
\hline Thiamine hydrochloride & 337 & 1 & 0.002967 \\
\hline Vitamin B12 & 1355 & 0.005 & 0.000004 \\
\hline i-Inositol & 180 & 35 & 0.194444 \\
\hline \multicolumn{4}{|l|}{ Inorganic Salts } \\
\hline Calcium nitrate $\left(\mathrm{Ca}(\mathrm{NO} 3)_{2} 4 \mathrm{H} 2 \mathrm{O}\right)$ & 236 & 100 & 0.423729 \\
\hline Magnesium Sulfate (MgSO4) (anhyd.) & 120 & 48.84 & 0.407 \\
\hline Potassium Chloride (KCl) & 75 & 400 & 5.333334 \\
\hline Sodium Bicarbonate ( $\mathrm{NaHCO} 3)$ & 84 & 2000 & 23.809525 \\
\hline Sodium Chloride ( $\mathrm{NaCl}$ ) & 58 & 6000 & 103.44827 \\
\hline $\begin{array}{l}\text { Sodium Phosphate dibasic (Na2HPO4) } \\
\text { anhydrous }\end{array}$ & 142 & 800 & 5.633803 \\
\hline Zinc sulfate $(\mathrm{ZnSO} 4-7 \mathrm{H} 2 \mathrm{O})$ & 288 & 0.874 & 0.003035 \\
\hline \multicolumn{4}{|l|}{ Proteins } \\
\hline AlbuMAX® II & & 400 & $\infty$ \\
\hline Human Transferrin (Holo) & & 7.5 & $\infty$ \\
\hline Insulin Recombinant Full Chain & & 10 & $\infty$ \\
\hline \multicolumn{4}{|l|}{ Trace Elements } \\
\hline Ammonium Metavanadate & 116.98 & 0.0003 & 0.000003 \\
\hline Cupric Sulfate & 250 & 0.00125 & 0.000005 \\
\hline Manganous Chloride & 198 & 0.00005 & 0 \\
\hline
\end{tabular}




\begin{tabular}{|l|r|r|r|}
\hline Sodium Selenite & 173 & 0.005 & 0.000029 \\
\hline Other Components & & & \\
\hline D-Glucose (Dextrose) & 180 & 2000 & 11.111111 \\
\hline Ethanolamine & 97.54 & 1.9 & 0.019479 \\
\hline Glutathione (reduced) & 307 & 1 & 0.003257 \\
\hline Phenol Red & 376.4 & 5 & 0.013284 \\
\hline Sodium Pyruvate & 110 & 110 & 1 \\
\hline
\end{tabular}

Related Products 


\title{
Resident Macrophages Initiating and Driving Inflammation in a Monosodium Urate Monohydrate Crystal-Induced Murine Peritoneal Model of Acute Gout
}

\author{
William John Martin, Michaela Walton, and Jacquie Harper
}

\begin{abstract}
Objective. To determine whether infiltrating monocytes, neutrophils, or resident macrophages contribute to the early inflammatory response to monosodium urate monohydrate (MSU) crystals in vivo.

Methods. MSU crystal-induced inflammation was monitored using a peritoneal model of acute gout. The production of proinflammatory cytokines

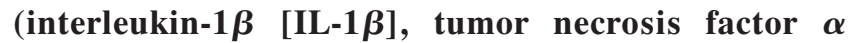
[TNF $\alpha]$, IL-6) by resident macrophages, infiltrating monocytes, and neutrophils during the onset of gout was determined by flow cytometry. Infiltrating and resident peritoneal cells were cultured with MSU crystals ex vivo, and proinflammatory cytokine production was determined by multiplex cytokine array. Activated macrophages on the visceral epithelial lining of the peritoneum were identified by immunofluorescence histochemistry. The inflammatory immune response to MSU crystals was then compared with the inflammatory response in mice depleted of resident macrophages by pretreatment with clodronate liposomes.

Results. The production of cytokines in vivo preceded the influx of $\mathrm{Gr}-1^{\text {intermediate }} 7 / 4+$ monocytes. Monocytes and neutrophils recruited during the inflammatory phase of the response to MSU crystals failed to produce proinflammatory cytokines either in vivo, or ex vivo following restimulation with MSU crystals. Stimulation of the naive peritoneal resident cell population with MSU crystals ex vivo resulted in positive staining
\end{abstract}

Supported by the Foundation for Research Science and Technology, New Zealand, and the Wellington Medical Research Fund, New Zealand.

William John Martin, MSc, Michaela Walton, PhD, Jacquie Harper, PhD: Malaghan Institute of Medical Research, Wellington, New Zealand.

Address correspondence and reprint requests to Jacquie Harper, PhD, Malaghan Institute of Medical Research, PO Box 7060, Wellington 6242, New Zealand. E-mail: jharper@malaghan.org.nz. Submitted for publication February 19, 2008; accepted in revised form September 25, 2008. of resident macrophages for the proinflammatory cytokines IL-1 $\beta$, TNF $\alpha$, and IL-6. Depletion of the resident macrophage population resulted in a significant decrease in both MSU crystal-induced neutrophil infiltration and proinflammatory cytokine production in vivo despite the presence of infiltrating monocytes.

Conclusion. These data indicate that resident macrophages, rather than infiltrating monocytes or neutrophils, are important for initiating and driving the early proinflammatory phase of acute gout.

Gout is an inflammatory arthritis induced by the precipitation of monosodium urate monohydrate (MSU) crystals in articular joints and periarticular tissues, where it presents as a severe acute inflammation that spontaneously resolves after 7-10 days $(1,2)$. The early inflammatory phase of acute gout is associated with the production of proinflammatory cytokines (interleukin-6 [IL-6], tumor necrosis factor $\alpha$ [TNF $\alpha$ ], IL-1 $\beta$ ) and the infiltration of leukocytes, including neutrophils and monocytes (3-6).

Previous in vitro and ex vivo studies indicate that the differentiation state of mononuclear phagocytes plays a key role in the type of cytokines produced in response to MSU crystals and therefore in the initiation and progression of an attack of gout. In those studies, monocyte-like cells were reported to produce proinflammatory cytokines such as TNF $\alpha$ and IL- $1 \beta$, whereas macrophage-like cells produced the antiinflammatory cytokine transforming growth factor $\beta$ (TGF $\beta$ ) (7-9). A similar pattern of proinflammatory to antiinflammatory cytokine production has been reported following differentiation of human CD14+ blood monocytes into macrophages (7). In addition, unpurified leukocyte infiltrates isolated from blisters during the course of an inflammatory response to cantharidin also exhibit cytokine "switching" following stimulation with MSU crystals in vitro (7). Together these data have led to the hypothesis 
that in an acute gout attack infiltrating monocytes drive the inflammatory response, while macrophages with a differentiated phenotype mediate the resolution of inflammation. As a result, previous gout research has mostly ignored the potential of resident macrophages to induce inflammation.

Recent literature now indicates a possible proinflammatory role of macrophages in gouty inflammation. Macrophages treated with MSU crystals have been reported to induce proinflammatory cytokines such as IL-1 $\beta(10,11)$, whereby signaling through the IL-1 receptor is required for activation of nonhemopoietic cells to induce neutrophilia (10), a key characteristic of gouty inflammation (12). Other proinflammatory activities such as the production of TNF $\alpha$, monocyte chemotactic protein 1, IL-18, and inducible nitric oxide synthase and the up-regulation of triggering receptor expressed on myeloid cells 1 (TREM-1) on macrophages provide evidence of the potential involvement of this cell type in gout (10,11,13-15). This would be consistent with findings in other acute inflammatory conditions that rely heavily on macrophages in the onset of inflammation $(16,17)$.

Both macrophages and monocytes appear to produce the key proinflammatory cytokines IL-1 $\beta$, IL-6, and $\mathrm{TNF} \alpha$ following in vitro stimulation with MSU crystals; however, the relevance of these findings with respect to the initiation and progression of inflammation in vivo has not been established. Therefore, studies that distinguish between the different phenotypes of mononuclear phagocyte populations over the course of the inflammatory response in vivo are needed to provide a more accurate depiction of the function of these cells in the context of gouty arthritis.

Although neutrophil infiltration is a hallmark feature of gout, little is known about the ability of these cells to subsequently respond to MSU crystals at the site of inflammation and produce IL- $1 \beta$, IL- 6 , or TNF $\alpha$ during the early phase of inflammation in vivo. Using a murine peritoneal model of acute gout, we have investigated the production of the gout-associated proinflammatory cytokines IL- $1 \beta$, IL- 6 , and TNF $\alpha$ by infiltrating neutrophils, monocytes, and resident macrophages during the initiation and early phase of MSU crystalinduced inflammation. Our results showed that resident macrophages, rather than infiltrating monocytes or neutrophils, were primarily responsible for the production of the proinflammatory cytokines IL- $1 \beta$ and IL- 6 and the infiltration of neutrophils in vivo during the onset of MSU crystal-induced inflammation. Contrary to the current dogma, infiltrating monocytes did not produce proinflammatory cytokines in response to MSU crystals in the initial stages of acute inflammation.

\section{MATERIALS AND METHODS}

Mice. Male C57BL/6 mice were bred and housed in a conventional animal facility at the Malaghan Institute of Medical Research, Wellington, New Zealand. All animals used for the experiments were age 8-10 weeks. All experimental procedures were approved by the Victoria University Animal Ethics Committee in accordance with their guidelines for the care of animals.

Reagents. Uric acid, lipopolysaccharide (LPS), and saponin were obtained from Sigma (Auckland, New Zealand). Cytokine Bead Array kits, chamber slides, GolgiStop, and phycoerythrin-conjugated anti-mouse IL-6 and allophycocyanin-conjugated anti-Gr-1 monoclonal antibodies were obtained from BD Biosciences (North Ryde, New South Wales, Australia). Anti-mouse IL-1 $\beta$, biotin-conjugated anti-mouse IL- $1 \beta$, anti-mouse TNF $\alpha$, anti-mouse IL-6, and biotinconjugated mouse anti-rat $\mathrm{IgG}$ monoclonal antibodies were obtained from eBioscience (San Diego, CA). Anti-F4/80 and fluorescein isothiocyanate (FITC)-conjugated 7/4 antibodies were obtained from Serotec (Oxford, UK). Bio-Plex multiplex arrays were purchased from Bio-Rad (Hercules, CA). The mouse IL-1 $\beta$ enzyme-linked immunosorbent assay (ELISA) kit was obtained from R\&D Systems (Minneapolis, MN). The Limulus amebocyte cell lysate assay kit was obtained from Associates of Cape Cod (East Falmouth, MA). Heparin was obtained from Mayne Pharma (Melbourne, Victoria, Australia). Low cell binding plates were purchased from Nunc (Rochester, NY). Diff-Quik was obtained from Dade Behring Diagnostics (Newark, DE). AnalySIS Life Science extended focal imaging software was obtained from Olympus (Auckland, New Zealand). All other products were obtained from Invitrogen (Auckland, New Zealand) unless otherwise stated.

Preparation of MSU crystals. MSU crystals were prepared by crystallization of a supersaturated solution of uric acid under mildly basic conditions. Briefly, $250 \mathrm{mg}$ uric acid was added to $45 \mathrm{ml}$ of double-distilled water containing $300 \mu \mathrm{l}$ of $5 \mathrm{M} \mathrm{NaOH}$, and the solution was boiled until the uric acid was dissolved. The solution was passed through a $0.2-\mu M$ filter, and $1 \mathrm{ml}$ of $5 M \mathrm{NaCl}$ was added to the hot solution, which was then stored at $26^{\circ} \mathrm{C}$. After 7 days the resulting MSU crystals were washed with ethanol and acetone. The resulting triclinic, needle-shaped MSU crystals were 5-25 $\mu \mathrm{m}$ in length and were birefringent to polarized light. All MSU crystals were determined to be endotoxin free $(<0.01 \mathrm{EU} / 10 \mathrm{mg})$ by Limulus amebocyte cell lysate assay.

MSU crystal-induced peritonitis. C57BL/6 mice were administered an intraperitoneal (IP) injection of a 3-mg slurry of MSU crystals in $0.5 \mathrm{ml}$ phosphate buffered saline (PBS). At different time points mice were euthanized by $\mathrm{CO}_{2}$ administration, and the peritoneal exudate cells were harvested by lavage with $3 \mathrm{ml}$ PBS containing 25 units/ml heparin and 10\% fetal bovine serum (FBS). Cells were retrieved from the lavage fluid and analyzed by flow cytometry and by Diff-Quik staining of cytospin samples. Lavage fluid was retained for cytokine assay. 
Thioglycolate-induced peritonitis. C57BL/6 mice were administered an IP injection of $4 \%$ thioglycolate $(0.5 \mathrm{ml})$. After 4 hours the mice were euthanized by $\mathrm{CO}_{2}$ administration, and the peritoneal exudate cells were harvested as described above for MSU crystal-induced peritonitis.

Purification of MSU crystal-elicited neutrophils. Mice were administered an IP injection of MSU crystals ( $3 \mathrm{mg}$ in 0.5 $\mathrm{ml}$ PBS). After 4 hours the mice were euthanized by $\mathrm{CO}_{2}$ administration, and the peritoneal exudate cells were harvested by lavage with $3 \mathrm{ml}$ PBS containing 25 units/ml heparin. Cells were retrieved from the lavage fluid and stained for F4/80, Gr-1, and 7/4. Neutrophils (F4/80-Gr-1 $\left.{ }^{\text {high }} 7 / 4+\right)$ were isolated by fluorescence-activated cell sorting (FACS) using a BD FACSVantage Diva (Becton Dickinson, San Jose, CA). Neutrophils were determined to be $>99 \%$ pure by flow cytometry and Diff-Quik analysis of cytocentrifuged samples. Neutrophil viability was confirmed to be $>99 \%$ by trypan blue exclusion.

Restimulation assay. Harvested peritoneal exudate cells were cultured in RPMI 1640 and $10 \%$ FBS at $1 \times 10^{6}$ cells $/ \mathrm{ml}$ in 96-well plates and treated with PBS, $200 \mu \mathrm{g} / \mathrm{ml}$ MSU crystals, or $100 \mathrm{ng} / \mathrm{ml}$ LPS for 16 hours at $37^{\circ} \mathrm{C}$. Supernatants were then harvested for cytokine analysis.

Cytokine analyses. Cytokine levels in harvested lavage PBS and from culture supernatants were assayed by Cytokine Bead Array and analyzed on a FACSCalibur flow cytometer (BD Biosciences), by Bio-Plex multiplex array and analyzed on a Bio-Plex flow cytometer, or by ELISA.

Intracellular staining. Total peritoneal exudate cells from mice treated with MSU crystals for 4 hours were harvested by peritoneal lavage and quickly suspended at $10^{6}$ cells/ml in RPMI 1640 containing 10\% FBS, PenStrep, Glutamax, and 1:1,500 GolgiStop and transferred into 24-well low cell binding plates. Positive controls were stimulated with 1 $\mu \mathrm{g} / \mathrm{ml}$ LPS, after which all cells were incubated for a further 4 hours at $37^{\circ} \mathrm{C}$ to allow for intracellular accumulation of cytokines. Cells were permeabilized with $0.1 \%$ saponin and stained for intracellular IL-6. The cells were washed and resuspended in FACS buffer, then stained for the surface markers F4/80, Gr-1, and 7/4. Cells were analyzed by flow cytometry using a FACSCalibur flow cytometer. Cytospin samples of exudate cells were also prepared by Diff-Quik staining to complement differential cell counts determined by flow cytometry.

Immunofluorescence. Resident peritoneal cells from naive mice were suspended at $10^{6}$ cells/ml in RPMI 1640 containing $10 \%$ FBS, PenStrep, and Glutamax and placed into 8 -well chamber slides. After incubation at $37^{\circ} \mathrm{C}$ for 1 hour, 1:1,500 GolgiStop was added. Cells were stimulated with 200 $\mu \mathrm{g} / \mathrm{ml} \mathrm{MSU}$ crystals, $1 \mu \mathrm{g} / \mathrm{ml}$ LPS, or PBS and incubated for 4 hours, then treated with zinc fixative for 30 minutes. Endogenous biotin was blocked using a biotin blocking kit (Invitrogen) in accordance with the manufacturer's instructions. Nonspecific $\mathrm{IgG}$ binding sites were then blocked using 5\% mouse serum or $5 \%$ FBS, followed by incubation overnight with anti-mouse IL-6, anti-mouse TNF $\alpha$, biotinylated anti-mouse IL- $1 \beta$, or the appropriate isotype control. Cells treated with anti-mouse IL- 6 or TNF $\alpha$ were then incubated with a biotinylated mouse anti-rat IgG antibody. Excess antibody was removed by washing with PBS, and the samples were stained with Alexa Fluor 555-conjugated streptavidin, FITC- conjugated anti-F4/80, and Hoechst 55542, mounted in Vectashield antifade (Vector, Burlingame, CA), and examined using a BX51 fluorescence microscope (Olympus, Tokyo, Japan).

Isolation of visceral peritoneal tissue. Mice were treated with an IP injection of $3 \mathrm{mg}$ MSU crystals as described above. After 4 hours the skin was removed from the abdomen, the peritoneum was cleared of leukocytes by lavage with $3 \mathrm{ml}$ PBS, and the ventral tissue covering the peritoneal cavity was excised and placed into cold RPMI 1640. The tissue was rinsed in PBS and fixed in acetone for 10 minutes at $-20^{\circ} \mathrm{C}$. The visceral lining of the peritoneum was harvested from the fixed tissue by carefully stripping the lining off as a single piece. The harvested visceral tissue was cleaned of fascia, and nonspecific IgG binding sites were blocked with a solution containing $10 \%$ FBS. Endogenous biotin was blocked using a kit. The tissue was stained for the surface markers 7/4 and F4/80 and mounted onto slides with Vectashield antifade. Slides were analyzed by fluorescence microscopy, and images were processed using AnalySIS Life Science extended focal imaging software.

Clodronate liposome depletion of resident macrophages. Clodronate liposomes were prepared as previously described (18). Mice were treated by IP injection of $200 \mu \mathrm{l}$ of either clodronate liposomes or PBS. Three days later, macrophage depletion in liposome-treated mice was confirmed by flow cytometry, following which mice were immediately challenged with an IP injection of $3 \mathrm{mg}$ of MSU crystals.

\section{RESULTS}

Profile of MSU crystal-induced inflammation in vivo. To identify the cell populations in the peritoneal lavage fluid of naive and MSU crystal-treated mice over time, cells were stained for the macrophage differentiation marker F4/80 and the myeloid differentiation antigens Gr-1 and 7/4 (Figure 1). Neutrophils were identified as $\mathrm{Gr}-1^{\text {high }} 7 / 4+$, and monocytes were identified as $\mathrm{Gr}-1^{\text {intermediate }} 7 / 4+$ cells expressing low levels of $\mathrm{F} 4 / 80$. Resident macrophages were identified as $\mathrm{F} 4 / 80^{\text {high }}$ cells staining negative for $\mathrm{Gr}-1$.

To establish the acute inflammatory profile of peritoneal inflammation in response to MSU crystals, we monitored leukocyte infiltration and the production of the proinflammatory cytokines IL- $1 \beta$, IL- 6 , and TNF $\alpha$ over 72 hours. Infiltration of both monocytes and neutrophils into the peritoneum was observed after 4 hours and peaked at 16 hours after injection of MSU crystals (Figure 2A). Neutrophil infiltration was notably faster and greater than that of monocytes. As shown in Figures 2B-D, IL-6, IL- $1 \beta$, and $\mathrm{TNF} \alpha$ levels were elevated in the peritoneum of MSU crystal-treated mice within 2 hours of administration of MSU crystals, peaking at 4 hours for all 3 cytokines. The inflammatory response was self limiting, with leukocyte cell numbers and cytokine levels returning to normal within 48 hours. 

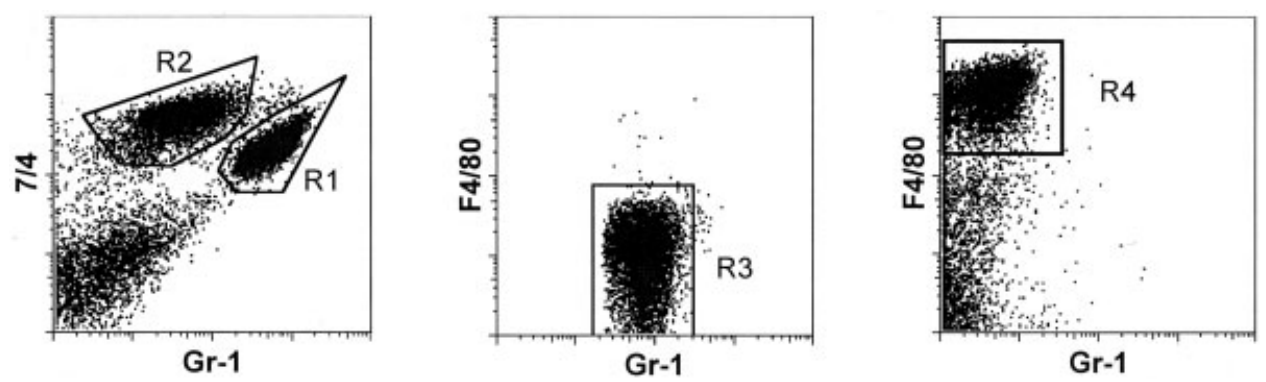

Figure 1. Identification of cell types involved in the monosodium urate monohydrate (MSU) crystal response in vivo. Mice were treated with MSU crystals intraperitoneally $(3 \mathrm{mg}$ in $0.5 \mathrm{ml}$ phosphate buffered saline [PBS]). After 4 hours peritoneal cells were harvested by lavage with $3 \mathrm{ml}$ PBS, and the cell types were identified by flow cytometry. Neutrophils were identified as Gr- $1^{\text {high }} 7 / 4+$ (gate R1), and monocytes were identified as Gr- $1^{\text {intermediate }} 7 / 4+$ cells (gate R2) expressing low levels of F4/80 (gate R3). Resident macrophages in untreated mice were identified as F4/80 ${ }^{\text {high }}$ cells staining negative for Gr- 1 (gate R4).

Based on peak cytokine production combined with early leukocyte infiltration, we chose to focus on the first 4-8 hours to determine which cell types (neutrophils, monocytes, and/or macrophages), were driving the early inflammatory response to MSU crystals.
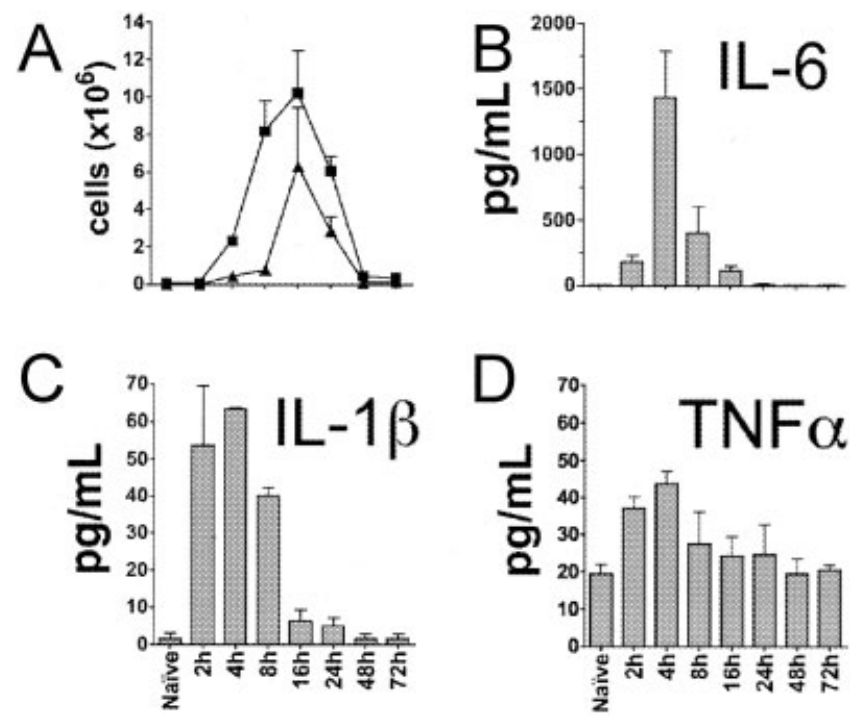

Figure 2. Monosodium urate monohydrate (MSU) crystals induce cellular infiltration and cytokine production in vivo. Mice were treated with MSU crystals intraperitoneally $(3 \mathrm{mg}$ in $0.5 \mathrm{ml}$ phosphate buffered saline [PBS]), and at different time points the peritoneal cells were harvested by lavage with $3 \mathrm{ml}$ PBS. A, Numbers of monocytes (triangles) and neutrophils (squares) infiltrating the peritoneum were determined by flow cytometry over 72 hours. B-D, Supernatants from the peritoneal lavage fluid were analyzed for interleukin-6 (IL-6) (B), IL-1 $\beta(\mathbf{C})$, and tumor necrosis factor $\alpha(\mathrm{TNF} \alpha)$ (D) using Cytokine Bead Arrays. Results are representative of 3 independent experiments. Values are the mean and SEM $(n=3$ mice per group).
Cytokine production by infiltrating monocytes and neutrophils. To investigate the potential contribution of infiltrating monocytes and neutrophils to early inflammation following exposure to MSU crystals, we collected peritoneal lavage fluid from mice 4 hours after treatment with MSU crystals and examined the capacity of infiltrating Gr- ${ }^{\text {intermediate }} 7 / 4+$ monocytes and Gr$1^{\text {high }} 7 / 4+$ neutrophils to produce the proinflammatory cytokine IL-6 4 hours after MSU crystal treatment (Figure 3A). Surprisingly, the infiltrating monocyte population did not produce IL-6 in response to MSU crystals, although monocytes were still able to produce inflammatory cytokines in response to LPS stimulation. Infiltrating neutrophils did not produce IL-6 in response to either MSU crystals or LPS (Figure 3A). In fact, none of the cells isolated from the peritoneal lavage fluid appeared to be producing large amounts of IL-6 (Figure $3 \mathrm{~A})$.

To confirm that the cells in the peritoneal exudate from MSU crystal-treated mice were unable to produce proinflammatory cytokines in response to MSU crystals, they were restimulated with MSU crystals ex vivo, and the supernatants were analyzed for the presence of IL-6, IL- $1 \beta$, and TNF $\alpha$. None of these cytokines was elevated following MSU crystal treatment (Figure 3B), indicating that infiltrating cells were not the source of cytokine production illustrated in Figure 2B. Serum components in cell culture have been shown to inhibit MSU crystal-induced neutrophil superoxide production (19) and could therefore affect neutrophil cytokine production. However, isolated neutrophils restimulated with MSU crystals also failed to produce IL-6, TNF $\alpha$, or IL- $1 \beta$ under low serum conditions (data 

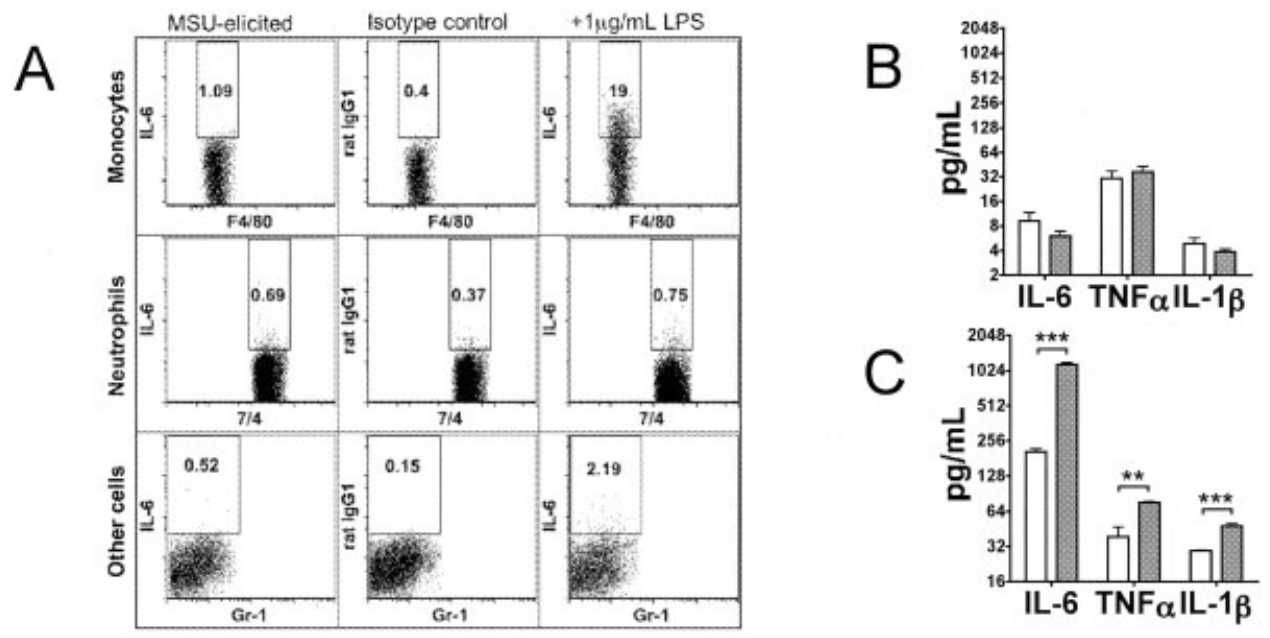

Figure 3. Infiltrating leukocytes do not produce inflammatory cytokines in response to monosodium urate monohydrate (MSU) crystals. Mice were treated with MSU crystals intraperitoneally ( $3 \mathrm{mg}$ in $0.5 \mathrm{ml}$ phosphate buffered saline [PBS]), and after 4 hours the peritoneal exudate cells were harvested by lavage with $3 \mathrm{ml}$ PBS. A, MSU crystal-elicited peritoneal cells were treated with GolgiStop alone or with lipopolysaccharide (LPS; $1 \mu \mathrm{g} / \mathrm{ml}$ ) and were analyzed by flow cytometry for the presence of interleukin- 6 (IL-6)-positive monocytes or neutrophils. B and C, Peritoneal cells were isolated from mice 4 hours after intraperitoneal administration of MSU crystals (B) or from untreated mice (C) and restimulated with 200 $\mu \mathrm{g} / \mathrm{ml}$ MSU crystals ex vivo. Shaded bars represent cells restimulated with MSU crystals; open bars represent PBS-treated controls. Supernatants from cell cultures were analyzed for IL-6, tumor necrosis factor $\alpha(\mathrm{TNF} \alpha)$, and IL-1 $\beta$ using Cytokine Bead Arrays. Values are the mean and SEM $(\mathrm{n}=3$ mice per group). Results are representative of 3 independent experiments. $* *=P<0.01 ; * * *=P<0.001$.

available online at http://www.malaghan.org.nz/research/ arthritis/arthritis-publications/), confirming that the lack of cytokine production was not serum dependent.

Monocytes recruited following IP administration of thioglycolate also failed to produce significant levels of inflammatory cytokines in response to restimulation with MSU crystals (data available online at http:// www.malaghan.org.nz/research/arthritis/arthritispublications/), indicating that the lack of inflammatory response to MSU crystals may be a common feature of newly recruited monocytes in acute inflammation. However, peritoneal cells from naive mice did produce significant amounts of inflammatory cytokines following exposure to MSU crystals ex vivo (Figure 3C), indicating that one or more of the resident cell populations was responsible for cytokine production following administration of MSU crystals.

Activation and adherence of resident macrophages following MSU crystal administration in vivo. The absence of cytokine-producing cells from the peritoneal lavage fluid of MSU crystal-treated mice led us to investigate changes in total cell numbers during the course of the inflammatory response to MSU crystals.
We observed an initial drop in the total number of peritoneal cells within the first 2 hours following administration of MSU crystals (Figure 4A) and before the infiltration of neutrophils and monocytes (Figure 2A). Between $60 \%$ and $70 \%$ of cells in the peritoneum of naive mice are resident macrophages; therefore, any significant drop in cell number is likely to be associated with a decrease in this population. Flow cytometric analysis of the $\mathrm{F} 4 / 80^{\text {high }} \mathrm{Gr}-1-7 / 4-$ resident macrophage population showed complete disappearance of this population from the peritoneal lavage fluid after 2 hours (Figure 4B).

Cell adhesion is a common feature of macrophage activation, and the absence of macrophages shortly after administration of inflammatory stimuli has been reported as the "macrophage disappearance reaction" in other models of acute inflammation (20-22). To confirm that macrophage adherence was occurring in vivo following administration of MSU crystals, we isolated visceral peritoneal membranes from mice 4 hours after MSU crystal administration and stained for the presence of $\mathrm{F} 4 / 80^{\text {high }} 7 / 4$ - resident macrophages, using immunofluorescence. Following MSU crystal treatment, 

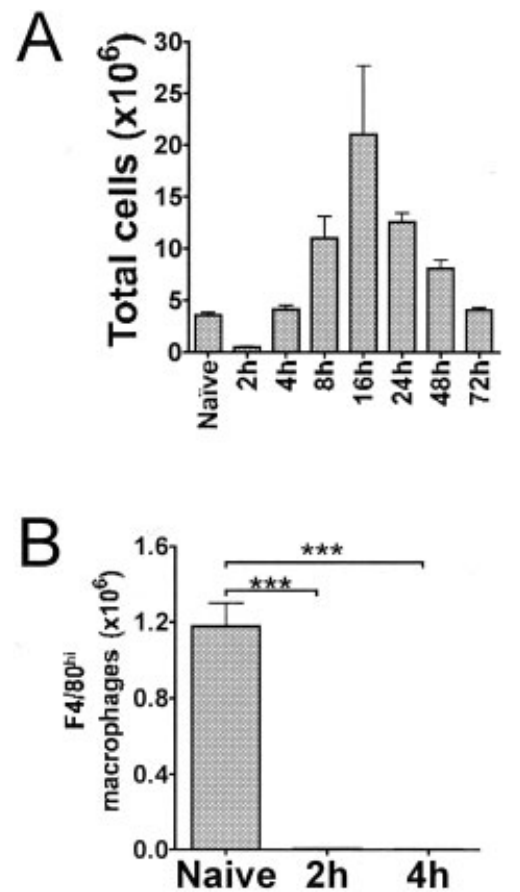

Figure 4. Disappearance of resident macrophages from peritoneal lavage fluid following administration of monosodium urate monohydrate (MSU) crystals. Mice were treated with MSU crystals intraperitoneally ( $3 \mathrm{mg}$ in $0.5 \mathrm{ml}$ phosphate buffered saline [PBS]), and at different time points the peritoneal cells were harvested by lavage with $3 \mathrm{ml}$ PBS. A, Total cell numbers were determined. B, Harvested peritoneal cells were analyzed by flow cytometry for the presence of $\mathrm{F} 4 / 80^{\text {high }}$ resident macrophages. Values are the mean and SEM $(\mathrm{n}=$ 3 mice per group). Results are representative of 3 independent experiments. $* * *=P<0.001$.
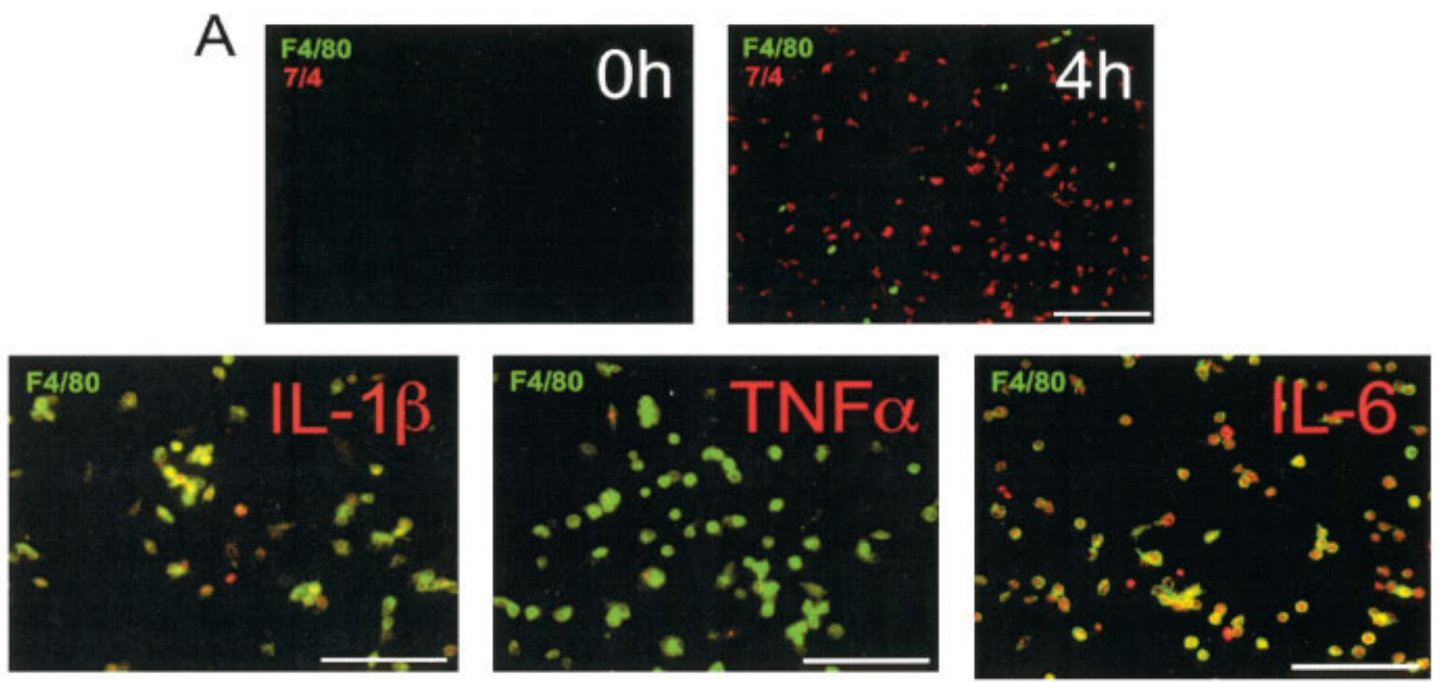

Figure 5. Resident macrophages adhere to the epithelial membrane and are a source of proinflammatory cytokines in response to monosodium urate monohydrate (MSU) crystals. A, Mice were treated with MSU crystals intraperitoneally ( $3 \mathrm{mg}$ in $0.5 \mathrm{ml}$ phosphate buffered saline), and after 4 hours the visceral epithelial lining of the peritoneum was harvested, fixed, and stained with anti-F4/80 (green) and 7/4 (red) to identify resident macrophages (F4/80+7/4-). B, Resident peritoneal cells were harvested from naive mice by lavage and incubated with $200 \mu \mathrm{g} / \mathrm{ml} \mathrm{MSU}$ crystals in the presence of GolgiStop for 4 hours. Cells were then stained with anti-F4/80 (green) to identify resident macrophages producing the following cytokines (red): interleukin-1 $\beta$ (IL-1 $\beta)$, tumor necrosis factor $\alpha(\mathrm{TNF} \alpha)$, and IL-6. Bars $=100 \mu \mathrm{m}$. Results are representative of 3 independent experiments. 

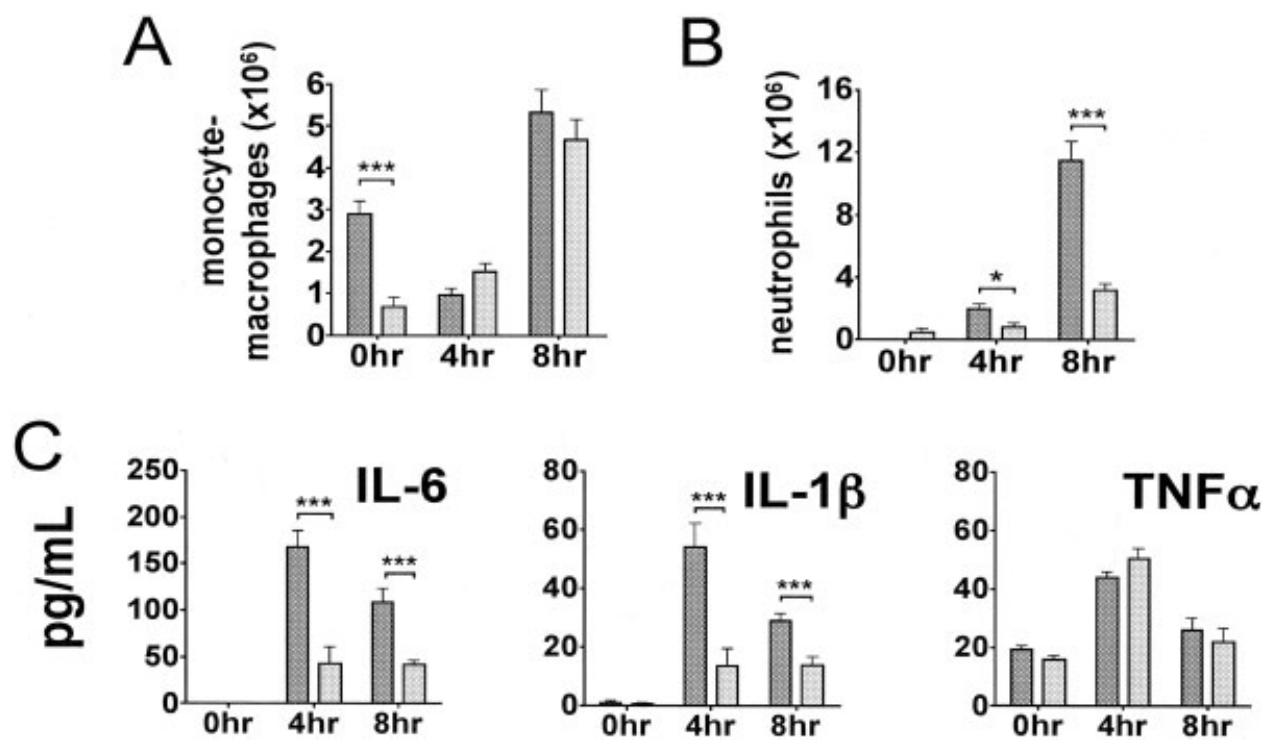

Figure 6. Depletion of resident macrophages inhibits monosodium urate monohydrate (MSU) crystalinduced neutrophil infiltration and proinflammatory cytokine production in vivo. Mice were not pretreated or were pretreated with clodronate liposomes to deplete peritoneal F4/80+ macrophages, 3 days prior to intraperitoneal administration of MSU crystals $(3 \mathrm{mg}$ in $0.5 \mathrm{ml}$ phosphate buffered saline [PBS]). At different time points, peritoneal cells were harvested by lavage with $3 \mathrm{ml}$ PBS and analyzed by flow cytometry for the presence of macrophages and monocytes (A) and neutrophils (B). Cytokine levels in the lavage fluid were measured by Bio-Plex multiplex array and by enzyme-linked immunosorbent assay (C). Light bars represent liposome-treated mice; dark bars represent PBS-treated control mice. Values are the mean and SEM ( $\mathrm{n}=5$ mice per group). Results are representative of 3 independent experiments. $*=P<0.05 ; * * *=P<0.001$. IL-6 = interleukin-6; TNF $\alpha=$ tumor necrosis factor $\alpha$.

phages play an essential role in the production of the key proinflammatory cytokines IL- 6 and IL- $1 \beta$ and in neutrophil recruitment in MSU crystal-induced inflammation.

\section{DISCUSSION}

Our results show that resident tissue macrophages, which have a highly differentiated phenotype, play a significant role in triggering acute MSU crystalinduced inflammation, whereby resident macrophages are a key source of proinflammatory cytokines including IL- $1 \beta$ and IL- 6 . In addition, the resident macrophage population appears to play an essential role in the initiation of neutrophil infiltration, as illustrated by the significant reduction in neutrophil infiltration following in vivo depletion of this population.

Once recruited to the site of inflammation, neither neutrophils nor monocytes appear to contribute to the proinflammatory cytokine production observed during the early stages of the acute inflammatory response to MSU crystals. The monocyte results were surprising in light of previous studies showing MSU crystal- induced proinflammatory cytokine production by monocytes treated with MSU crystals in vitro and by leukocyte infiltrates from cantharidin-induced blisters treated with MSU crystals ex vivo (7). However, in addition to using a different inflammatory stimulus for recruitment of cells, the cantharidin study investigated later-stage cellular infiltrates ( 16 hours compared with $4-8$ hours) that likely differ in cellular composition and phenotype compared with the initiation phase of MSU crystal-induced inflammation. In fact, phenotypic differences between in vivo MSU crystal-recruited monocytes and isolated monocytes and monocytic cell lines may also explain why our results differ from earlier in vitro data.

The lack of proinflammatory response to MSU crystals does not arise as a result of an inability to produce proinflammatory cytokines, since MSU crystalelicited monocytes readily produce IL-6 in response to LPS. The difference in responsiveness of infiltrating monocytes to MSU crystals and LPS suggests that the proinflammatory response to MSU crystals, unlike that to LPS, does not occur via Toll-like receptor 4 (TLR-4). This is consistent with earlier work showing that knock- 
ing out the gene encoding TLR-4 does not ablate the inflammatory response to MSU crystals (10). In addition, thioglycolate-elicited cells are also unresponsive to MSU crystal restimulation, showing that desensitization to MSU is unlikely to be the reason for the absence of cytokine production. In fact, the lack of the proinflammatory response to MSU crystal restimulation may be a common phenotype of early infiltrating monocytes regardless of how they are elicited.

Our study pinpoints the resident macrophage population as a primary source of the proinflammatory cytokines IL- $1 \beta$ and IL- 6 in the early response to MSU crystal exposure. Although the resident macrophages produce $\mathrm{TNF} \alpha$ in response to exposure to MSU crystals, they do not appear to be an essential source of TNF $\alpha$ in vivo. Our findings are consistent with a key involvement of resident macrophages in the initiation of other forms of acute inflammation $(16,17,23)$.

Although earlier in vitro studies have indicated that differentiated macrophages produce "antiinflammatory" cytokines such as TGF $\beta$ following exposure to MSU crystals, more recent reports have shown that MSU crystals can activate differentiated macrophages, leading to the activation of the NALP3 inflammasome, the production of IL- $1 \beta$, the up-regulation of TREM-1, and the production of cytokine-induced neutrophil chemoattractant (also called KC or CXCL1) $(10,11,13)$. These later findings support our in vivo results showing that the resident macrophage population initiates inflammation in response to MSU crystals. In our model it appears that MSU crystal-activated resident macrophages adhere to the surrounding tissues, produce proinflammatory cytokines, and initiate recruitment of neutrophils from the blood during the early inflammatory response.

The proinflammatory response of the resident macrophage population to MSU crystals does not preclude differentiated macrophages from playing a role in the resolution of inflammation at a later point in time. The plasticity of the macrophage phenotype is well known, and macrophage switching from a "proinflammatory" to an "antiinflammatory" phenotype over time remains a viable mechanism of action for resolution of acute inflammation. However, TGF $\beta$-producing macrophages have yet to be identified in vivo.

In part, the lack of proinflammatory cytokine production by infiltrating monocytes in response to MSU crystals could contribute to the self-limiting nature of the disease. Classically, infiltrating monocytes would augment and further prolong or amplify an inflammatory response by producing increased amounts of proin- flammatory cytokines. Since recruited monocytes did not exhibit a proinflammatory response to MSU crystals in our model, it is also possible that augmentation of the early inflammatory response to MSU crystals by infiltrating monocytes does not occur in gout.

Mononuclear phagocytes are highly diverse in phenotype and function, making them difficult to model faithfully in vitro. Although care needs to be taken when extrapolating findings from the peritoneal model of inflammation to joint inflammation in gout, the unmanipulated peritoneal environment allows for the complex interplay between resident and infiltrating immune cells, which is not provided by in vitro studies. Therefore, the in vivo model provides greater insight into the true effector phenotypes in the context of the inflammation that occurs in acute gout. To this end, this approach has shown that infiltrating monocytes elicited by MSU crystals in vivo behave differently from monocytes derived by other methods.

The immune response to MSU crystals is multifaceted in nature, making it difficult to account for all aspects of the response in one study. Although macrophages play a pivotal role, they obviously do not control the entire inflammatory response to MSU crystals. For example, macrophage activation and neutrophil infiltration do not appear to be a requirement for monocyte infiltration in our model, highlighting another aspect of the inflammatory response to MSU crystals that warrants further investigation. Taken together, our findings bring us a step closer to clearly identifying which immune cells are likely to be critically involved in different aspects of early inflammation in gout.

In summary, our work identifies resident macrophages, not infiltrating monocytes or neutrophils, as triggering and driving early MSU crystal-induced inflammation in the context of IL- $1 \beta$ and IL- 6 production and the initiation of neutrophil infiltration. Based on these findings, macrophages are also likely to be key cells involved in initiating and driving inflammation in gouty arthritis, suggesting the need to revisit the current understanding of induction of acute gout.

\section{AUTHOR CONTRIBUTIONS}

Dr. Harper had full access to all of the data in the study and takes responsibility for the integrity of the data and the accuracy of the data analysis.

Study design. Martin, Harper.

Acquisition of data. Martin, Walton.

Analysis and interpretation of data. Martin, Walton, Harper.

Manuscript preparation. Martin, Harper.

Statistical analysis. Martin. 


\section{REFERENCES}

1. Wallace SL, Robinson H, Masi AT, Decker JL, McCarty DJ, Yu TF. Preliminary criteria for the classification of the acute arthritis of primary gout. Arthritis Rheum 1977;20:895-900.

2. Gordon T, Terkeltaub R, Ginsberg MH. Gout: crystal-induced inflammation. In: Gallin JI, Goldstein IM, Snyderman R, editors. Inflammation: basic principles and clinical correlates. New York: Raven Press; 1988. p. 977-81.

3. Guerne PA, Terkeltaub R, Zuraw B, Lotz M. Inflammatory microcrystals stimulate interleukin-6 production and secretion by human monocytes and synoviocytes. Arthritis Rheum 1989;32: 1443-52.

4. Di Giovine FS, Malawista SE, Thornton E, Duff GW. Urate crystals stimulate production of tumor necrosis factor $\alpha$ from human blood monocytes and synovial cells: cytokine mRNA and protein kinetics, and cellular distribution. J Clin Invest 1991;87: 1375-81.

5. Di Giovine FS, Malawista SE, Nuki G, Duff GW. Interleukin 1 (IL 1) as a mediator of crystal arthritis: stimulation of $T$ cell and synovial fibroblast mitogenesis by urate crystal-induced IL 1 . J Immunol 1987;138:3213-8.

6. Schiltz C, Liote F, Prudhommeaux F, Meunier A, Champy R, Callebert J, et al. Monosodium urate monohydrate crystal-induced inflammation in vivo: quantitative histomorphometric analysis of cellular events. Arthritis Rheum 2002;46: 1643-50.

7. Yagnik DR, Evans BJ, Florey O, Mason JC, Landis RC, Haskard DO. Macrophage release of transforming growth factor $\beta 1$ during resolution of monosodium urate monohydrate crystal-induced inflammation. Arthritis Rheum 2004;50:2273-80.

8. Landis RC, Yagnik DR, Florey O, Philippidis P, Emons V, Mason JC, et al. Safe disposal of inflammatory monosodium urate monohydrate crystals by differentiated macrophages. Arthritis Rheum 2002;46:3026-33.

9. Yagnik DR, Hillyer P, Marshall D, Smythe CD, Krausz T, Haskard DO, et al. Noninflammatory phagocytosis of monosodium urate monohydrate crystals by mouse macrophages: implications for the control of joint inflammation in gout. Arthritis Rheum 2000;43: 1779-89.

10. Chen CJ, Shi Y, Hearn A, Fitzgerald K, Golenbock D, Reed G, et al. MyD88-dependent IL-1 receptor signaling is essential for gouty inflammation stimulated by monosodium urate crystals. J Clin Invest 2006;116:2262-71.

11. Martinon F, Petrilli V, Mayor A, Tardivel A, Tschopp J. Gout- associated uric acid crystals activate the NALP3 inflammasome. Nature 2006;440:237-41.

12. Phelps P, McCarty DJ Jr. Crystal-induced inflammation in canine joints. II. Importance of polymorphonuclear leukocytes. J Exp Med 1966;124:115-26.

13. Murakami Y, Akahoshi T, Hayashi I, Endo H, Kawai S, Inoue M, et al. Induction of triggering receptor expressed on myeloid cells 1 in murine resident peritoneal macrophages by monosodium urate monohydrate crystals. Arthritis Rheum 2006;54:455-62.

14. Jaramillo M, Naccache PH, Olivier M. Monosodium urate crystals synergize with IFN- $\gamma$ to generate macrophage nitric oxide: involvement of extracellular signal-regulated kinase $1 / 2$ and NF- $\kappa$ B. J Immunol 2004;172:5734-42.

15. Jaramillo M, Godbout M, Naccache PH, Olivier M. Signaling events involved in macrophage chemokine expression in response to monosodium urate crystals. J Biol Chem 2004;279:52797-805.

16. Knudsen E, Iversen PO, van Rooijen N, Benestad HB. Macrophage-dependent regulation of neutrophil mobilization and chemotaxis during development of sterile peritonitis in the rat. Eur J Haematol 2002;69:284-96.

17. Cailhier JF, Partolina M, Vuthoori S, Wu S, Ko K, Watson S, et al. Conditional macrophage ablation demonstrates that resident macrophages initiate acute peritoneal inflammation. J Immunol 2005;174:2336-42.

18. Van Rooijen N, Sanders A, van den Berg TK. Apoptosis of macrophages induced by liposome-mediated intracellular delivery of clodronate and propamidine. J Immunol Methods 1996;193: 93-9.

19. Terkeltaub R, Curtiss LK, Tenner AJ, Ginsberg MH. Lipoproteins containing apoprotein $\mathrm{B}$ are a major regulator of neutrophil responses to monosodium urate crystals. J Clin Invest 1984;73: 1719-30.

20. Nelson DS, Boyden SV. The loss of macrophages from peritoneal exudates following the injection of antigens into guinea-pigs with delayed-type hypersensitivity. Immunology 1963;6:264-75.

21. Haskill S, Becker S. Disappearance and reappearance of resident macrophages: importance in C. parvum-induced tumoricidal activity. Cell Immunol 1985;90:179-89.

22. Melnicoff MJ, Horan PK, Morahan PS. Kinetics of changes in peritoneal cell populations following acute inflammation. Cell Immunol 1989;118:178-91.

23. Richards PJ, Williams AS, Goodfellow RM, Williams BD. Liposomal clodronate eliminates synovial macrophages, reduces inflammation and ameliorates joint destruction in antigen-induced arthritis. Rheumatology (Oxford) 1999;38:818-25. 